\title{
APERTURE-TOLERANT, CHEMICAL-BASED METHODS TO REDUCE CHANNELING
}

Final Scientific/Technical Progress Report, Reporting Period: October 1, 2004 through May 16, 2007

Principal Author: $\quad$ Randall S. Seright

Report Date: $\quad$ May 16, 2007

DOE Award Number: $\quad$ DE-FC26-04NT15519

Name and Address of Submitting Organization:

New Mexico Petroleum Recovery Research Center

New Mexico Institute of Mining and Technology

Socorro, New Mexico 87801

PRRC Report 07-05 


\section{DISCLAIMER}

This report was prepared as an account of work sponsored by an agency of the United States Government. Neither the United States Government nor any agency thereof, nor any of their employees, makes any warranty, expressed or implied, or assumes any legal liability or responsibility for the accuracy, completeness, or usefulness of any information, apparatus, product, or process disclosed, or represents that its use would not infringe privately owned rights. Reference herein to any specific commercial product, process, or service by trade name, trademark, manufacturer, or otherwise does not necessarily constitute or imply its endorsement, recommendation, or favoring by the United States Government or any agency thereof. The views and opinions of authors expressed herein do not necessarily state or reflect those of the United States Government or any agency thereof.

\section{DEDICATION}

This report is dedicated to Dr. Jerry Casteel, who recently retired after many years of government service. During his time with the DOE's National Petroleum Technology Office, Jerry consistently played an extremely constructive part in overseeing our research. While our government is often criticized for wasteful spending and flighty funding of ill-considered projects, Jerry was a great role model, illustrating the very best of our public servants. Being highly competent (both technically and managerially), he provided a voice of moderation and charted a steady long-term course for solving some of the nation's most difficult energy

problems. We will miss his insights, his vision, his dedication to advancing petroleum engineering, and his friendship. 


\begin{abstract}
This final technical progress report describes work performed from October 1, 2004, through May 16, 2007, for the project, "Aperture-Tolerant, Chemical-Based Methods to Reduce Channeling."
\end{abstract}

We explored the potential of pore-filling gels for reducing excess water production from both fractured and unfractured production wells. Several gel formulations were identified that met the requirements - i.e., providing water residual resistance factors greater than 2,000 and ultimate oil residual resistance factors $\left(F_{r r o}\right)$ of 2 or less. Significant oil throughput was required to achieve low $F_{\text {rro }}$ values, suggesting that gelant penetration into porous rock must be small (a few feet or less) for existing pore-filling gels to provide effective disproportionate permeability reduction. Compared with adsorbed polymers and weak gels, strong pore-filling gels can provide greater reliability and behavior that is insensitive to the initial rock permeability.

Guidance is provided on where relative-permeabiliy-modification/disproportionate-permeabilityreduction treatments can be successfully applied for use in either oil or gas production wells. When properly designed and executed, these treatments can be successfully applied to a limited range of oilfield excessive-water-production problems.

We examined whether gel rheology can explain behavior during extrusion through fractures. The rheology behavior of the gels tested showed a strong parallel to the results obtained from previous gel extrusion experiments. However, for a given aperture (fracture width or plate-plate separation), the pressure gradients measured during the gel extrusion experiments were much higher than anticipated from rheology measurements. Extensive experiments established that wall slip and first normal stress difference were not responsible for the pressure gradient discrepancy. To explain the discrepancy, we noted that the aperture for gel flow (for mobile gel wormholing through concentrated immobile gel within the fracture) was much narrower than the width of the fracture.

The potential of various approaches were investigated for improving sweep in parts of the Daqing Oil Field that have been EOR targets. Possibilities included (1) gel treatments that are directed at channeling through fractures, (2) colloidal dispersion gels, (3) reduced polymer degradation, (4) more viscous polymer solutions, and (5) foams and other methods. Fractures were present in a number of Daqing wells (both injectors and producers). Because the fractures were narrow far from the wellbore, severe channeling did not occur. On the contrary, fractures near the wellbore aided reservoir sweep.

In the February 2006 issue of the Journal of Petroleum Technology, a "Distinguished-AuthorSeries" paper claimed that a process using aqueous colloidal dispersion gels (CDG gels) performed superior to polymer flooding. Unfortunately, this claim is misleading and generally incorrect. Colloidal dispersion gels, in their present state of technological development, should not be advocated as an improvement to, or substitute for, polymer flooding. 
TABLE OF CONTENTS

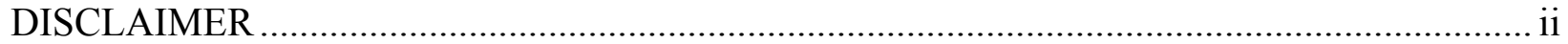

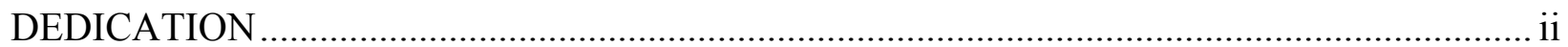

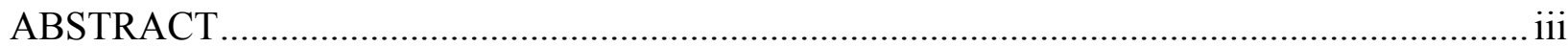

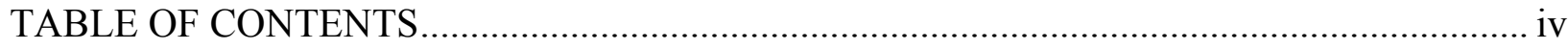

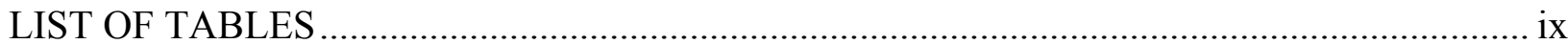

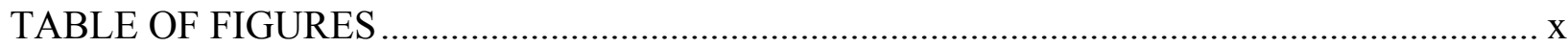

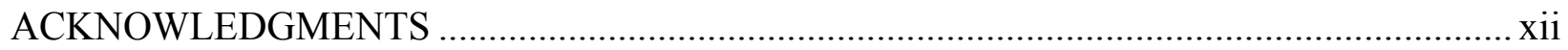

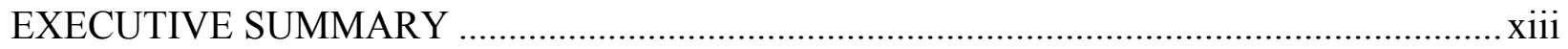

Optimizing Disproportionate Permeability Reduction ................................................... xiii

Guidelines for Relative Permeability Modification Treatments .........................................xiii

Correlating Gel Rheology with Behavior during Extrusion .............................................. xiv

Sweep Improvement Options for the Daqing Oil Field ................................................... xiv

Are Colloidal Dispersion Gels Really a Viable Technology? .............................................. Xv

Can Foams Provide Superior Mobility Control Compared with Polymers? ........................... XV

How is Vertical Sweep Affected When Resistance Factors Vary with Permeability?............ xvi

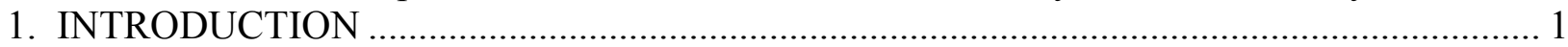

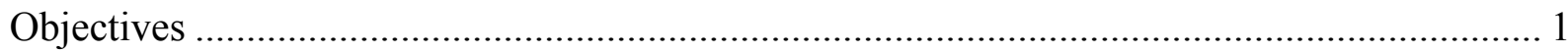

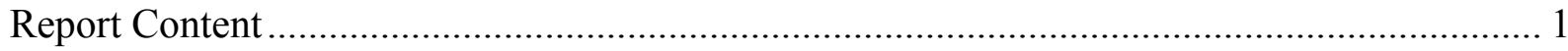

2. OPTIMIZING DISPROPORTIONATE PERMEABILITY REDUCTION ........................... 2

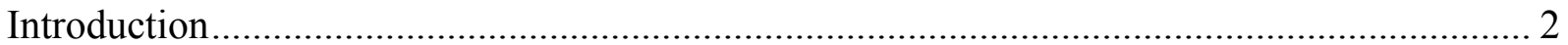

Challenges for Applications of Disproportionate Permeability Reduction ............................. 2

Variable Performance ............................................................................................ 2

$F_{\text {rro }}$ Must Be $<2$ for Radial Flow ............................................................................ 2

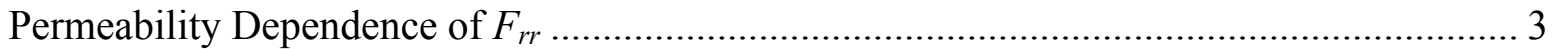

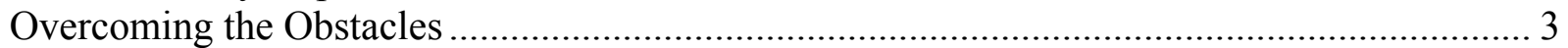

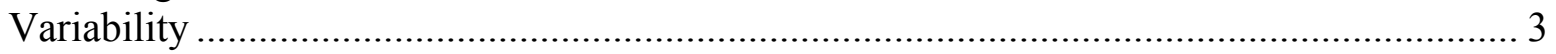

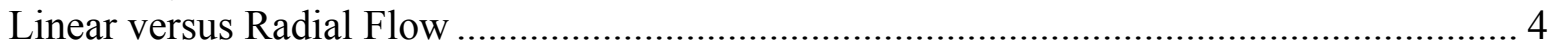

Permeability Dependence ......................................................................................... 5

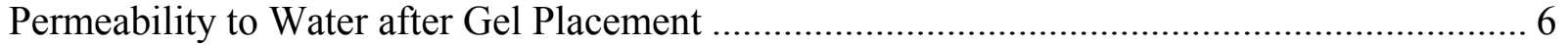

$k_{w}$ versus Initial Core Permeability and Core Material .................................................. 6

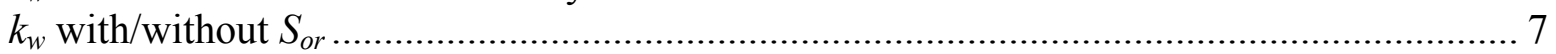

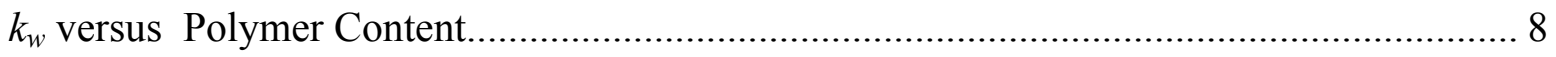

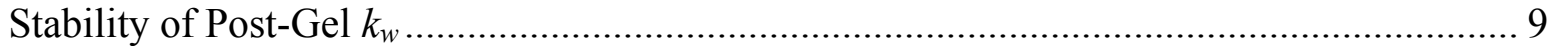

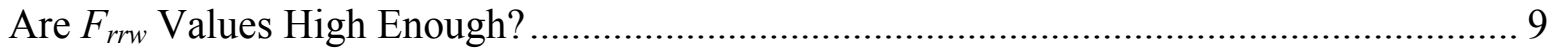

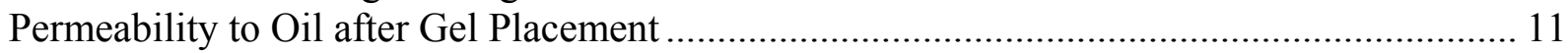

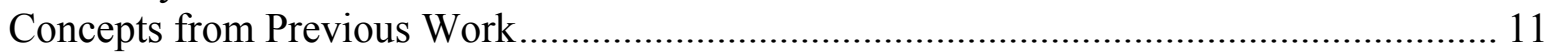

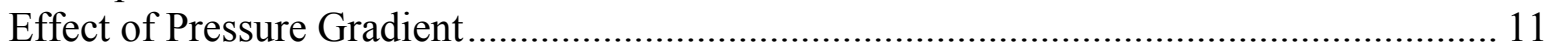

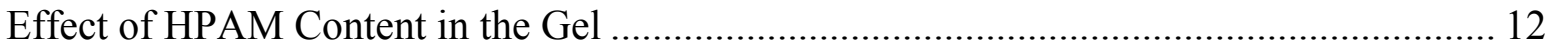

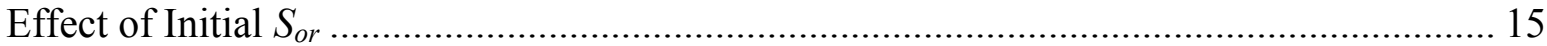

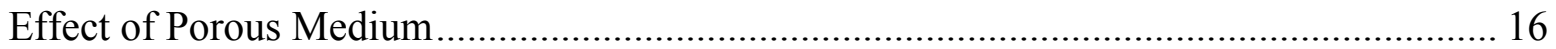

Effect of Polymer Type in the Gel .............................................................................. 17

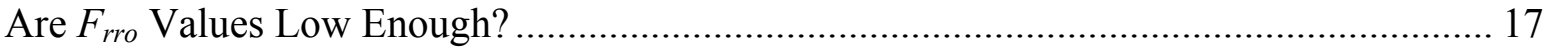

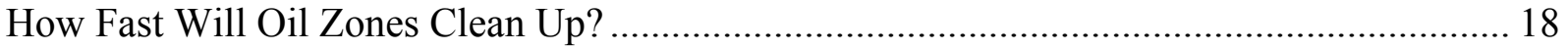

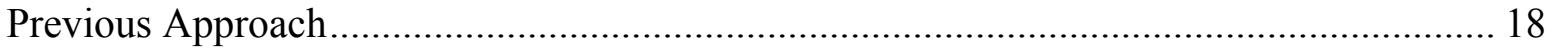




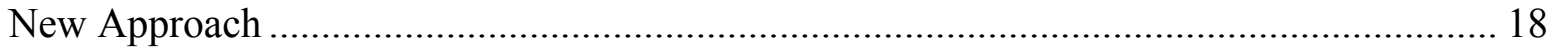

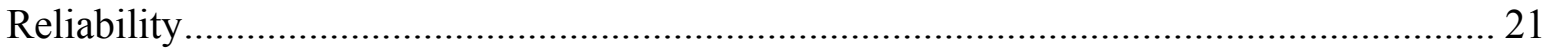

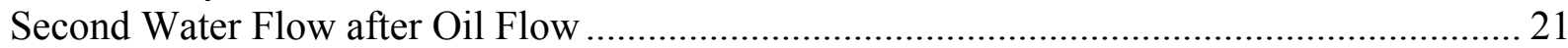

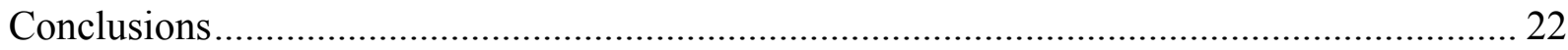

3. GUIDELINES FOR RELATIVE PERMEABILITY MODIFICATION TREATMENTS ..... 23

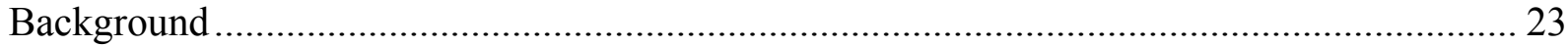

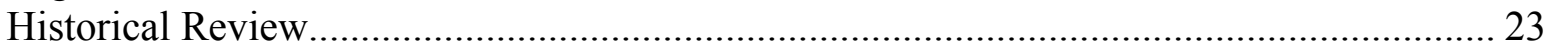

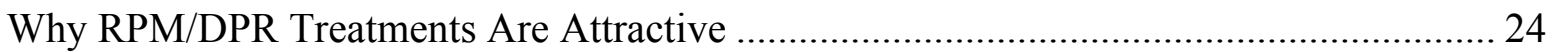

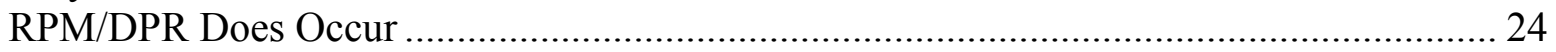

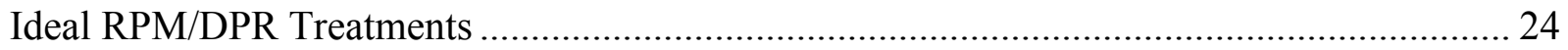

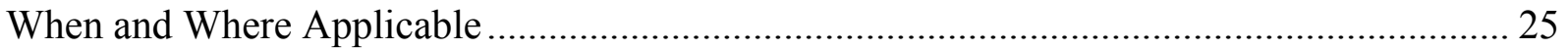

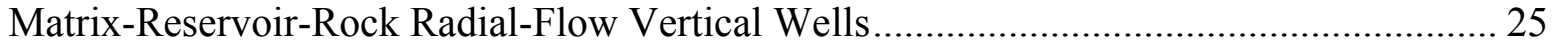

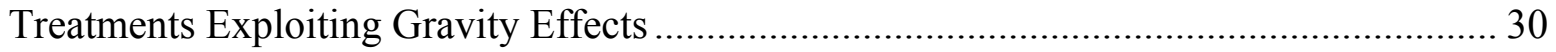

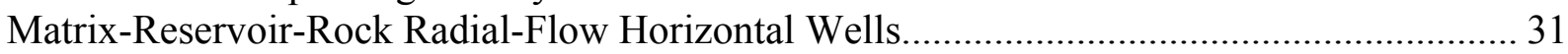

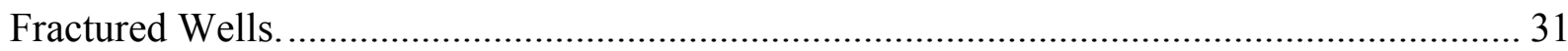

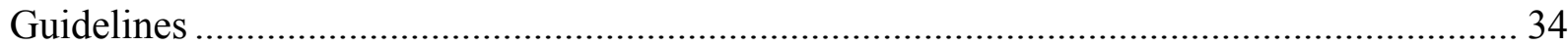

Treatment Limitations and Potential Pitfalls ........................................................................ 36

Treatments for Matrix-Reservoir-Rock Radial Flow Wells ............................................... 36

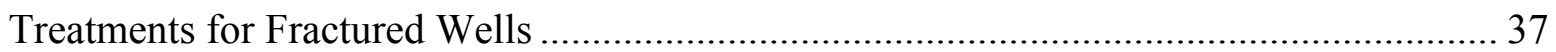

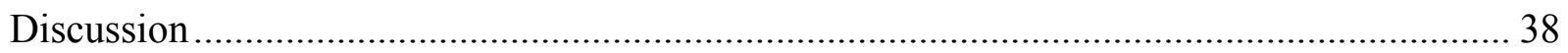

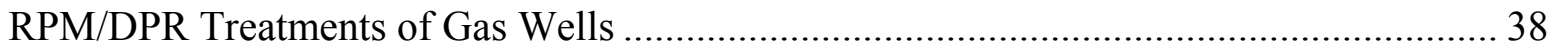

Determine or Deduce the Water-Production Problem ........................................................ 38

Need for Custom Engineering ......................................................................................... 39

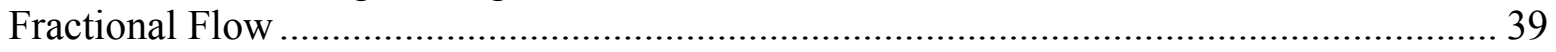

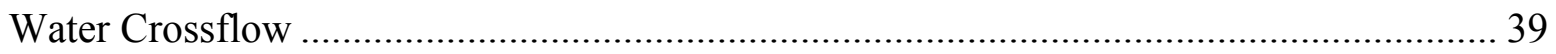

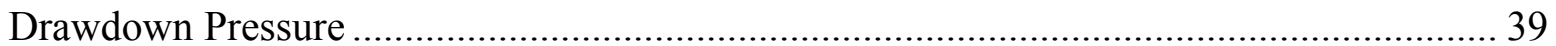

DPR \& Reduction in Water-Producing Rate Do Not Necessarily Correlate ........................ 40

Issue of Possibly Shutting Off Oil Production.................................................................. 40

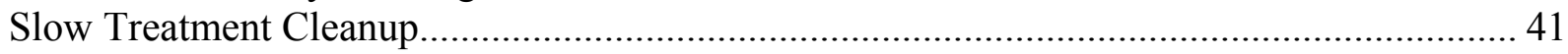

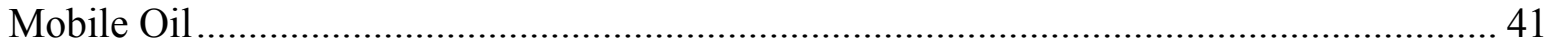

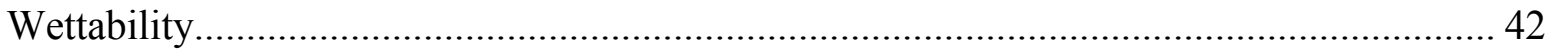

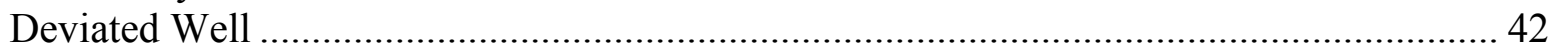

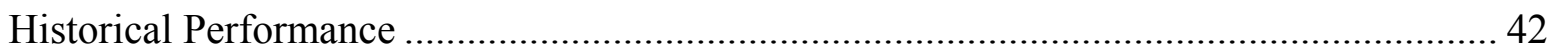

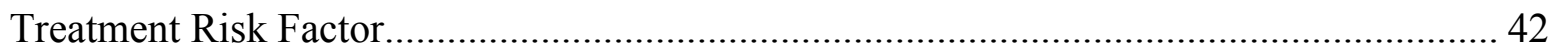

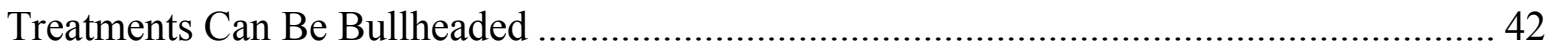

Treatment Development \& Exploitation Activity........................................................... 42

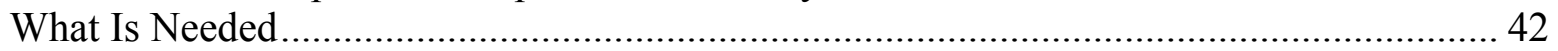

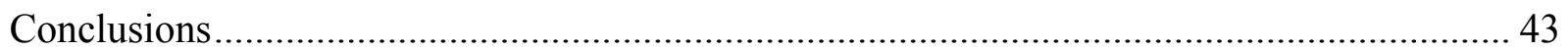

4. CORRELATING GEL RHEOLOGY WITH BEHAVIOR DURING EXTRUSION ............. 45

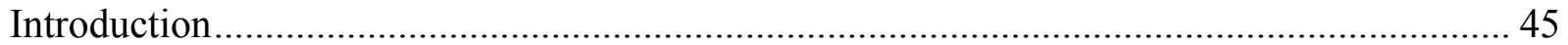

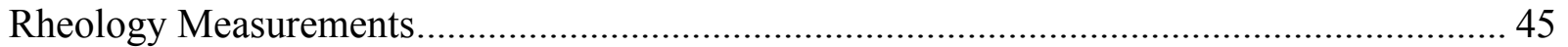

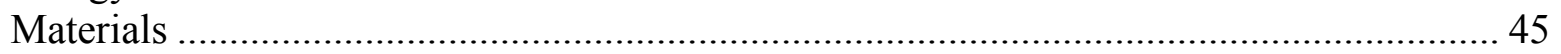

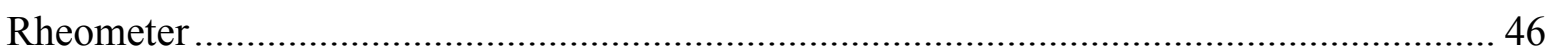

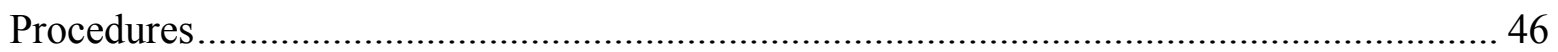

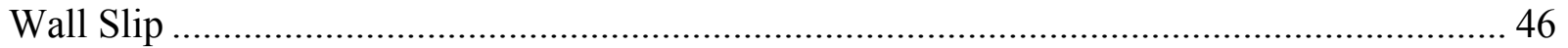

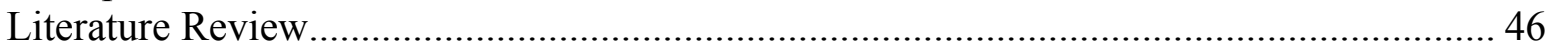




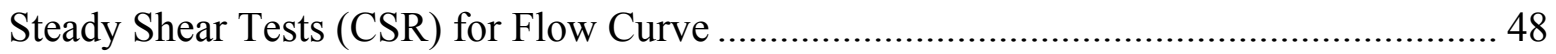

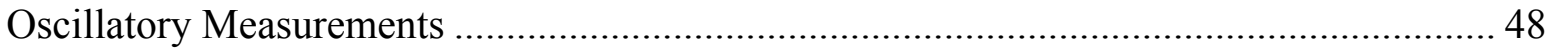

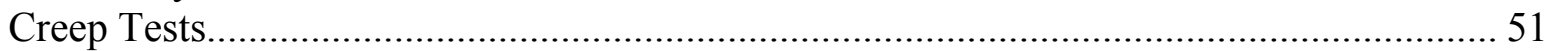

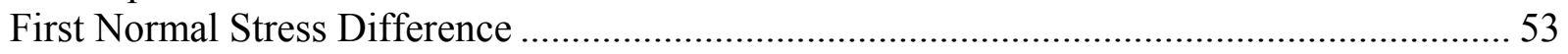

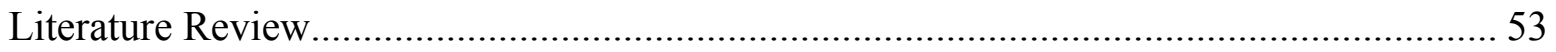

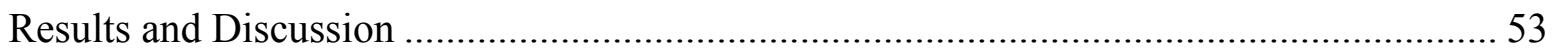

Gel Dehydration and Wormholes in Fractures ………….................................................. 55

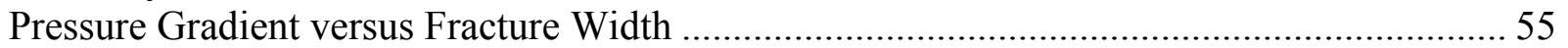

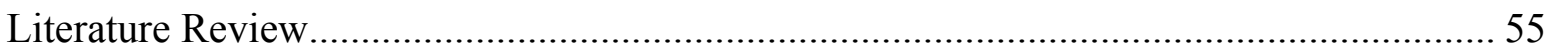

Model Based on Shell Momentum Balance and Power-Law ……………………….......... 57

Pressure Gradient versus Flow Rate and Shear Stress versus Shear Rate ............................ 61

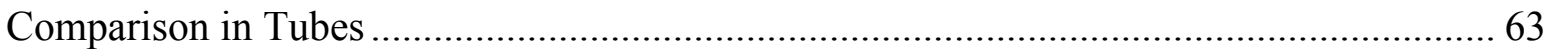

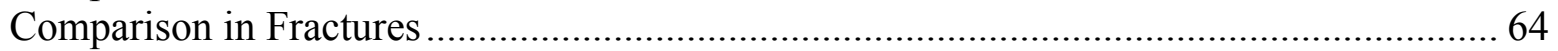

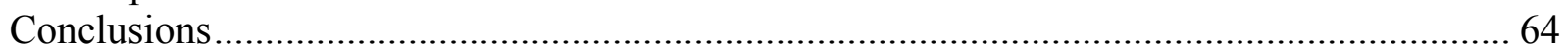

5. SWEEP IMPROVEMENT OPTIONS FOR THE DAQING OIL FIELD ………..................... 66

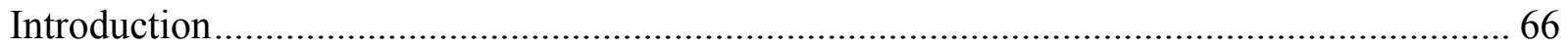

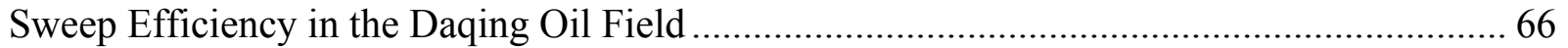

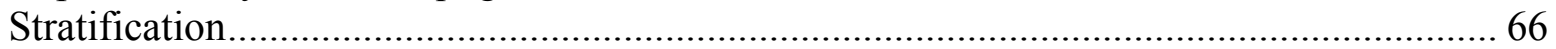

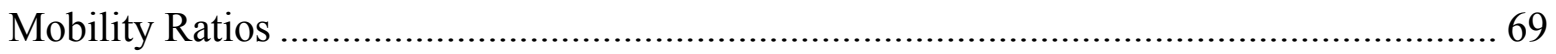

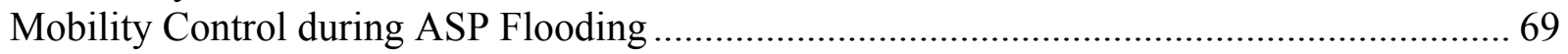

Mobility Control Requirements for ASP Flooding after Polymer Flooding ........................ 69

Higher Salinity of ASP Reduces Viscosity ..................................................................... 70

Polymer Stability in the Presence of Alkali ................................................................... 71

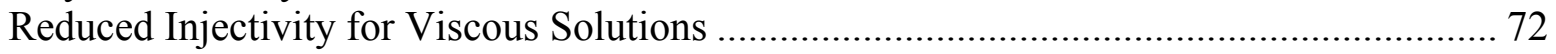

Are Fractures Important? Are Gels Viable to Reduce Channeling?........................................ 74

Pattern Oil Recovery Factors ................................................................................... 74

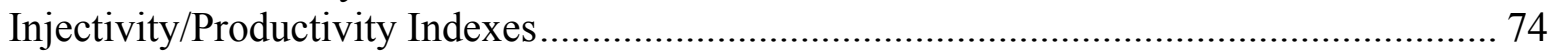

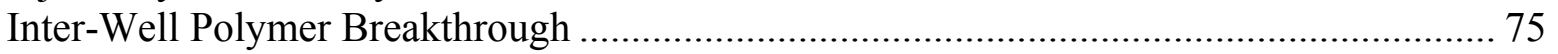

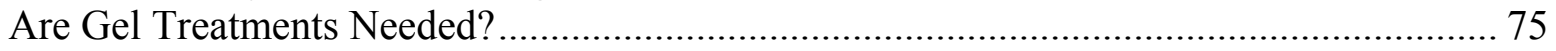

Are "Colloidal Dispersion Gels" a Viable Option? ................................................................. 78

Laboratory Behavior of Colloidal Dispersion Gels ......................................................... 79

Requirements for a Viable Colloidal Dispersion Gel ......................................................... 80

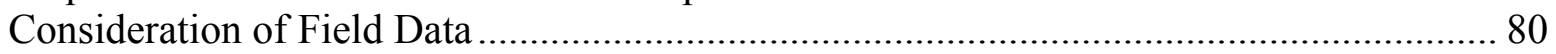

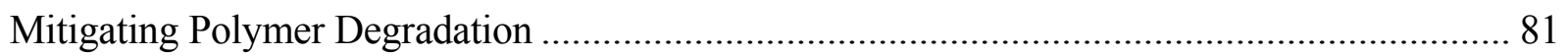

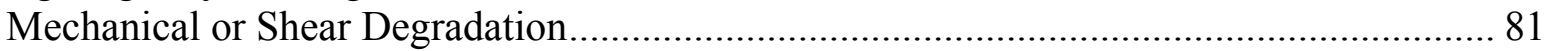

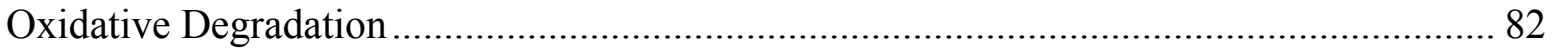

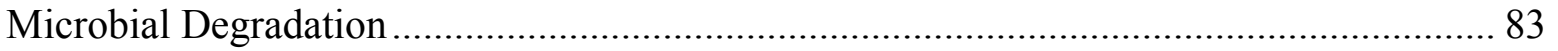

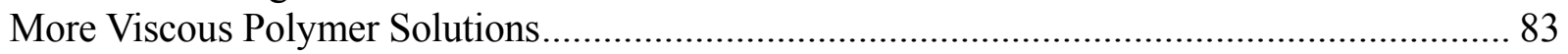

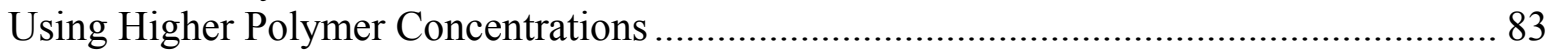

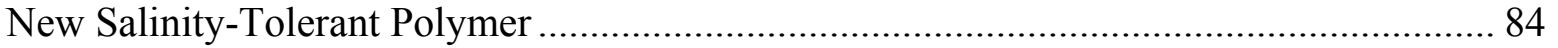

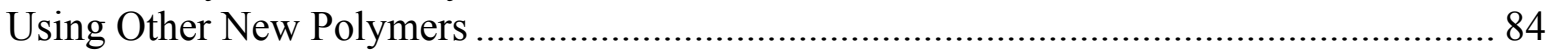

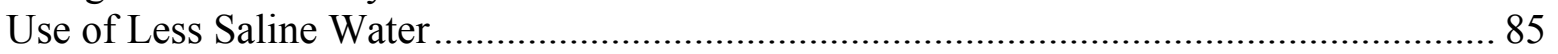

Are Foams a Viable Option? ......................................................................................... 85

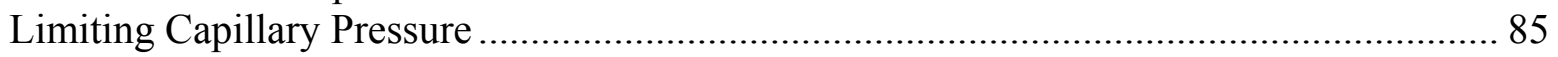

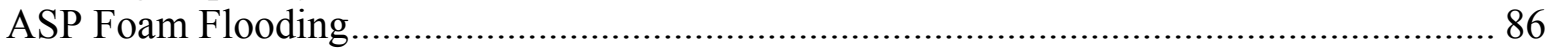

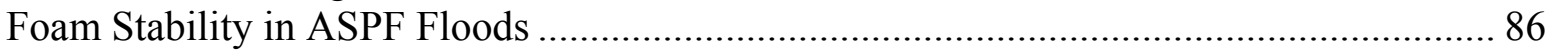




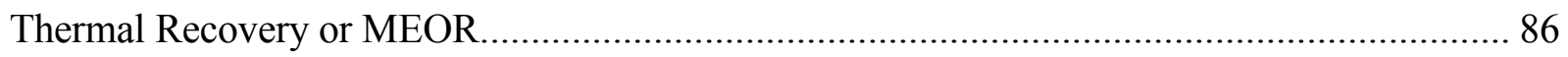

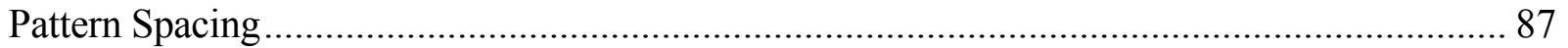

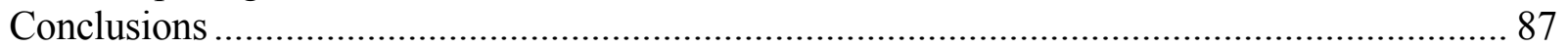

6. ARE COLLOIDAL DISPERSION GELS REALLY A VIABLE TECHNOLOGY? .............. 88

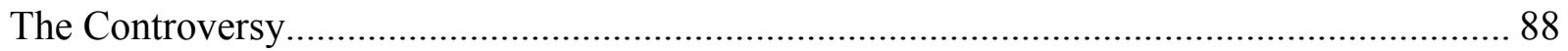

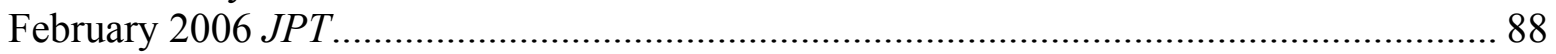

Controversy Background. ..................................................................................... 88

Polymers and Gelants Can Flow Through Rock. Gels Do Not. .......................................... 89

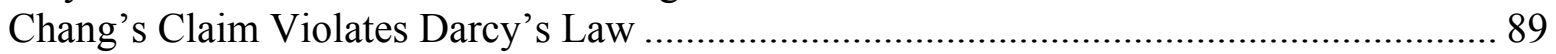

Polymers and Gels Plug Low-k Rock More than High-k Rock............................................ 90

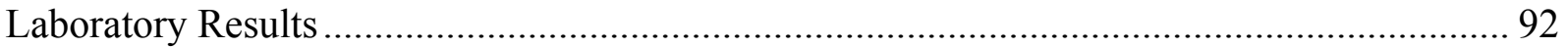

Many Previous Lab Tests Show No Effect of Aluminum on Polymer............................... 92

Other Lab Tests Show Permeability Reductions, But Gelation Times Are Short ................ 92

Parallel Corefloods Are a Poor Indicator of Diversion...................................................... 92

The Vendor's Lab Tests Show No Surprises................................................................. 92

Requirements for a Viable Colloidal Dispersion Gel ...................................................... 93

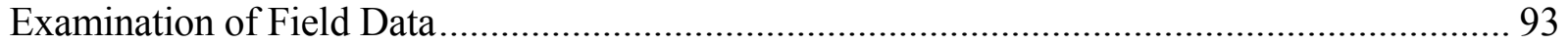

The Field and Lab Data Are Right. Only Interpretations Are Wrong ................................. 93

Field Results Can Be Explained Using More Plausible Concepts.......................................... 93

Daqing Field Results Are Consistent with No Benefit from Aluminum Citrate .................. 94

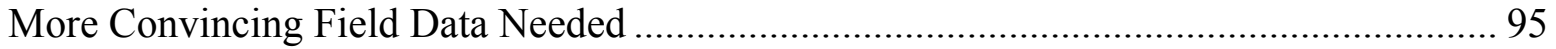

Searching for a New Mechanism of Action for CDG Gels .................................................... 95

Does Shearing Of Colloidal Dispersion Gels Allow For Effective, Deep Penetration?....... 95

Can CDG Gels Flow in Porous Media with Permeabilities Less than 8 Darcys? ................ 99

Can Low-Concentration Gels Be More Effective than Normal Gels in Tight Fractures?...... 101

Behavior during Gel Injection .................................................................................... 103

Behavior during Brine Injection after Gel Placement..................................................... 104

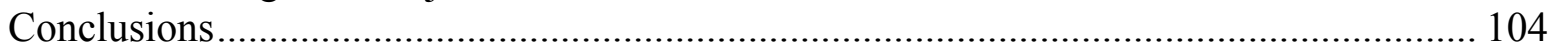

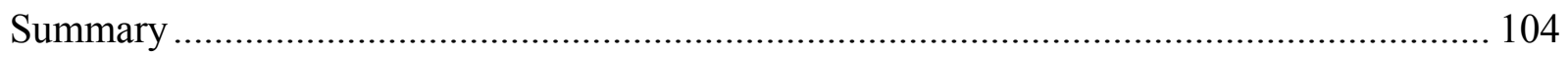

7. CAN FOAMS BE SUPERIOR TO POLYMERS FOR IMPROVING VERTICAL SWEEP

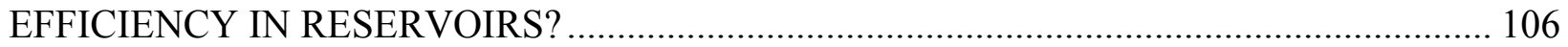

Normal Behavior of Viscous Fluids in Layered Reservoirs with No Crossflow.................... 106

Normal Behavior of Viscous Fluids in Layered Reservoirs with Crossflow......................... 107

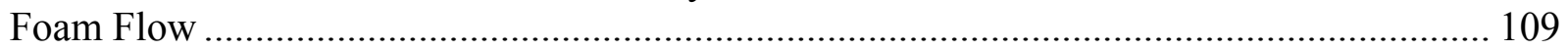

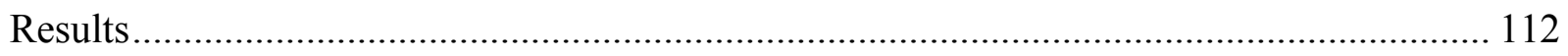

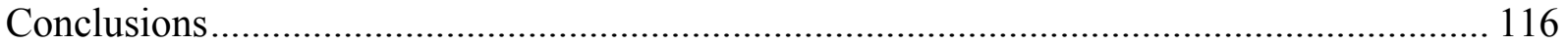

8. HOW IS VERTICAL SWEEP AFFECTED WHEN RESISTANCE FACTORS VARY WITH

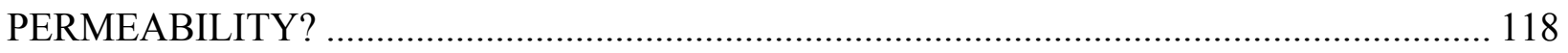

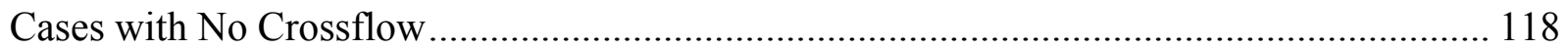

Same Resistance Factor in Both Zones.......................................................................... 119

Linear Flow, Different Resistance Factor in the Zones ...................................................... 119

Radial Flow, Different Resistance Factor in the Zones ............................................... 119

Comparison with Laboratory Data.............................................................................. 119

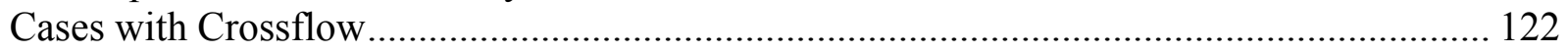

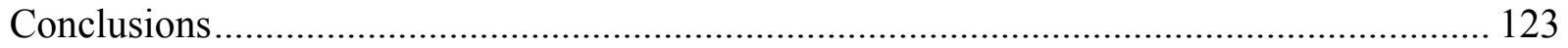

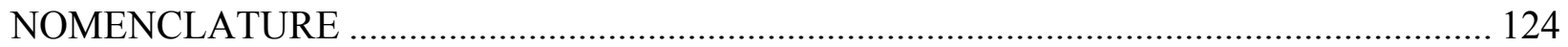




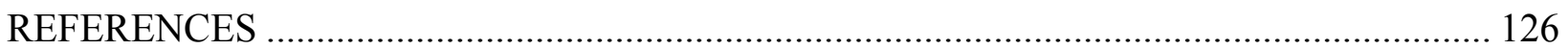

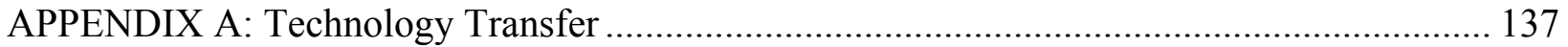

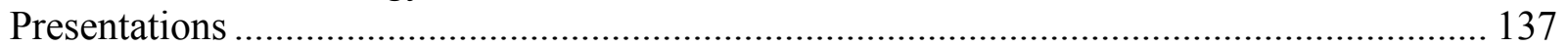

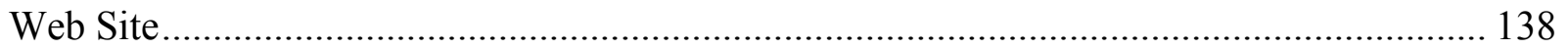

Papers and Publications .......................................................................................... 139

APPENDIX B: Derivation of Penetration Equations for Floods with Newtonian Fluids .......... 141

APPENDIX C: Derivation of Penetration Equations for Unstable Displacement in Zone 2 ..... 145 


\section{LIST OF TABLES}

Table $1-k_{w}$ during brine flow after gel placement ................................................................

Table 2-Ultimate $k_{o}$ and $F_{\text {rro }}$ during oil flow after gel placement .........................................12

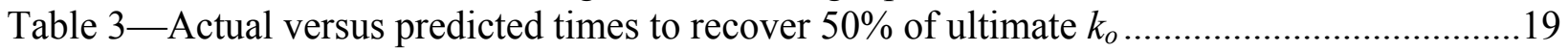

Table 4-Ultimate $k_{w}$ and $F_{r r w}$ during the second water flow after gel placement .......................22

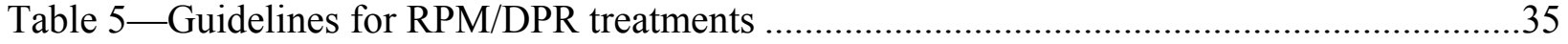

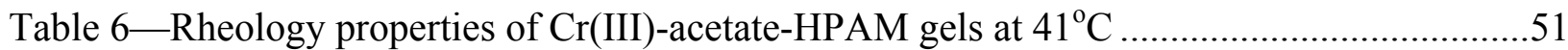

Table 7-Pressure gradient versus tube radius/fracture width for Cr(III)-acetate-HPAM gels ....60

Table 8 - Correlation of pressure gradient, shear stress, flow rate, and shear rate at the wall......63

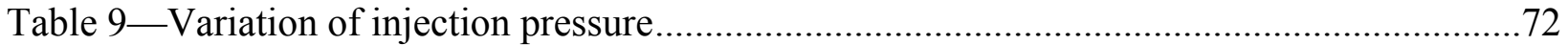

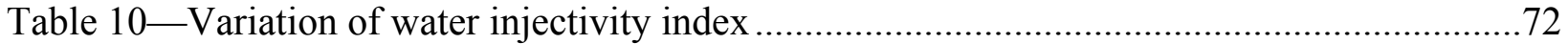

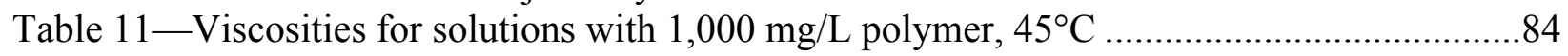

Table 12-Viscous solutions penetrate proportionately more into low-k zones ............................90

Table 13 - Individual zone flow capacities: after/before gel placement........................................91

Table 14-Use of low-concentration gels in tight fractures .......................................................102 


\section{TABLE OF FIGURES}

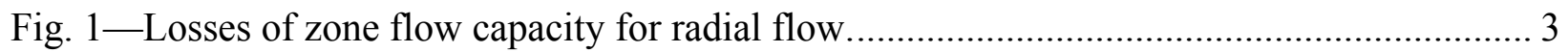

Fig. 2-Gel restricting water entry into a fracture................................................................. 4

Fig. 3-Permeabilities to oil and water after gel placement......................................................... 5

Fig. 4-Variations in $k_{w}$ for pore-filling gels versus weak gels. .................................................. 6

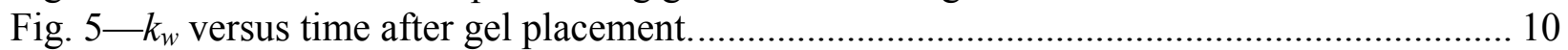

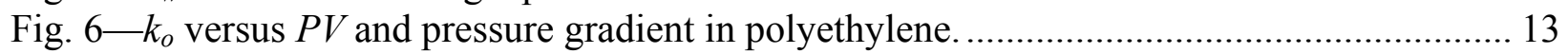

Fig. 7 $k_{o}$ versus $P V$ and pressure gradient in Berea sandstone............................................... 13

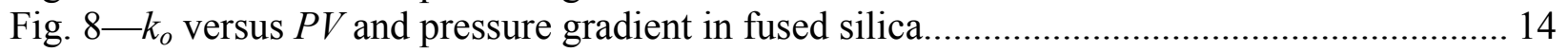

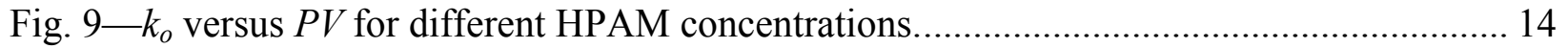

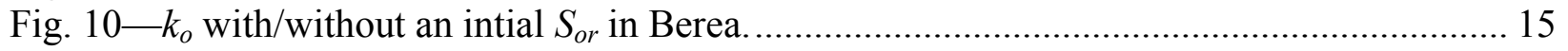

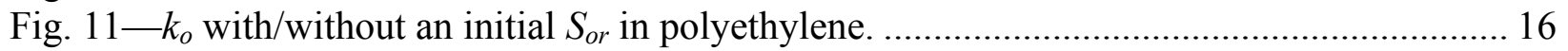

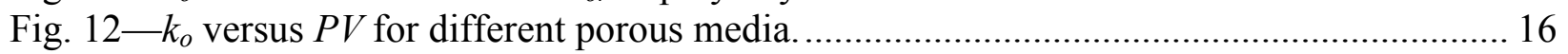

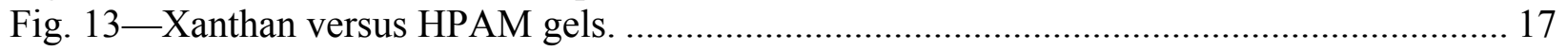

Fig. 14-Predicted clean up times: old versus new models. ..................................................... 20

Fig. 15-DPR treatment in a single strata producing at a fixed fractional flow. .......................... 26

Fig. 16 -DPR treatment in a reservoir with crossflow............................................................... 27

Fig. 17-DPR treatment in a reservoir having a dry-oil producing strata with crossflow........... 27

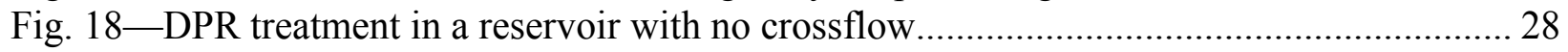

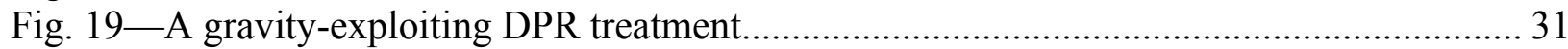

Fig. 20-Use of DPR to inhibit water entry into a fracture or fracture system ............................. 32

Fig. 21-Flow curves for $1 \mathrm{X}, 2 \mathrm{X}$, and $3 \mathrm{X}$ gels on smooth surfaces.......................................... 49

Fig. 22-Flow curve comparison of $1 \mathrm{X}$ and $2 \mathrm{X}$ gels on different surfaces. .............................. 50

Fig. 23-Dynamic property comparison for a $1 \mathrm{X}$ gel versus gap on different surfaces. .............. 50

Fig. 24-Creep tests for $1 \mathrm{X}, 2 \mathrm{X}$, and $3 \mathrm{X}$ gels on MP31, double-rough surface at $41^{\circ} \mathrm{C} \ldots \ldots \ldots \ldots . . .52$

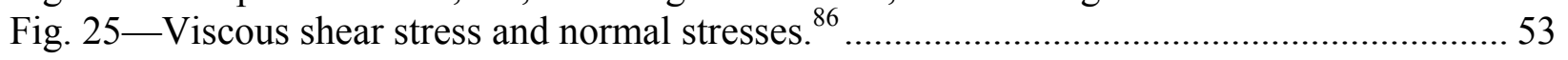

Fig. 26-First normal stress difference versus shear rate for $1 \mathrm{X}, 2 \mathrm{X}$, and $3 \mathrm{X}$ gels at $41^{\circ} \mathrm{C}$....... 54

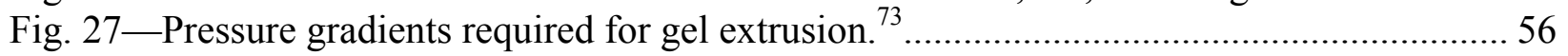

Fig. 28 - Comparison of pressure gradients versus aperture for $1 \mathrm{X}$ gel......................................... 60

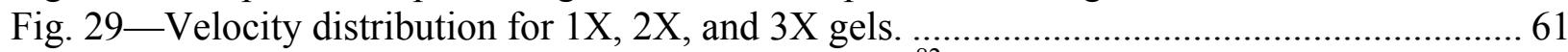

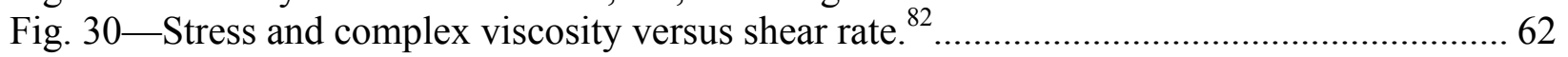

Fig. 31-Comparison of shear stress versus shear rate for $1 \mathrm{X}$ gel in tubes. ...............................63

Fig. 32-Comparison of shear stress versus shear rate for 1X gel in fractures............................ 64

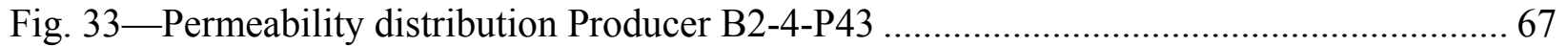

Fig. 34-Permeability distribution Producer B2-D6-P45 ……………......................................... 68

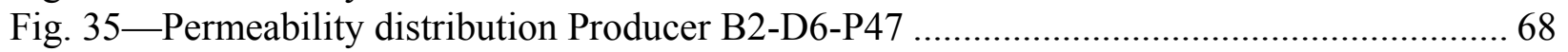

Fig. 36-Viscosity of medium molecular weight polymer (LIANHUA-1500) ……................... 70

Fig. 37-Viscosity of ultra-high molecular weight polymer (HENGJU)..................................... 71

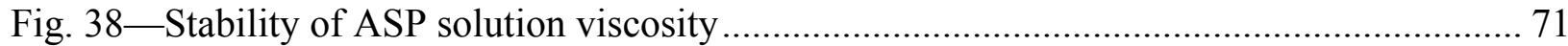

Fig. 39-Pattern pressures before ASP: Boundary is $10 \mathrm{MPa}$................................................. 73

Fig. 40 - Pattern pressures after ASP: Boundary is $12 \mathrm{MPa}$.................................................... 73

Fig. 41 -Pressure distribution with a 1 -mm wide fracture in $0.259-\mu \mathrm{m}^{2}$ rock ............................ 76

Fig. 42 — Pressure distribution with no fracture present .......................................................... 77

Fig. $43-w_{f}=1 \mathrm{~mm}$ for the $1 \mathrm{st}$ and $3 \mathrm{rd} 100-\mathrm{m}$ fracture sections; $w_{f}=0.15 \mathrm{~mm}$ in 2 nd section.. 77 
Fig. 44-Polymer solution viscosities at various points during injection .............................. 82

Fig. 45-Distinction between a gel treatment and a polymer flood...................................... 89

Fig. 46- $F_{r r}$ versus $k$ for an HPAM solution. ................................................................... 90

Fig. 47-Resistance factors in the first short core during injection at a high rate..................... 96

Fig. 48-Resistance factors in the first long core during injection at a low rate........................ 97

Fig. 49-Resistance factors in the second short core during injection at a high rate ................. 98

Fig. 50-Resistance factors in the second long core during injection at a low rate................... 99

Fig. 51-Resistance factors for a 1-day-old CDG gel in a 7.9-darcy core ............................. 100

Fig. 52-Viscosity versus shear rate for HPAM solutions (no crosslinker)............................ 102

Fig. 53-Pressure gradients versus volume throughput in tight fractures.............................. 103

Fig. 54-Linear flow, no crossflow, unit-mobility displacement...................................... 107

Fig. 55-Linear flow, no crossflow, normal Newtonian viscous fluid. ................................ 107

Fig. 56 - Radial flow, no crossflow, Newtonian and non-Newtonian polymeric fluids. .......... 107

Fig. 57-Linear flow, free crossflow, unit-mobility displacement. ....................................... 108

Fig. 58 - Radial flow, free crossflow, unit-mobility displacement. ..................................... 108

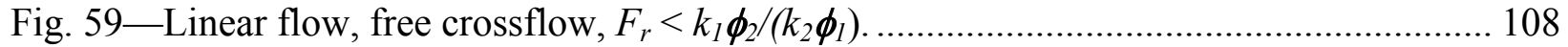

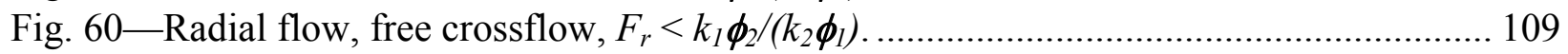

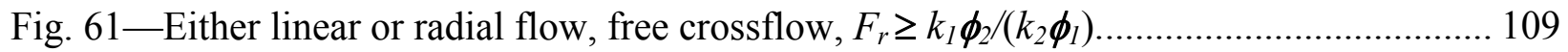

Fig. 62-Foam forms in Zone 1 but not in Zone 2, free crossflow....................................... 110

Fig. 63-Incorrect view of gas/water flow in Zone 2 after foam forms in Zone 1................. 110

Fig. 64-Gas/water flow in Zone 2 after foam forms in Zone 1. No Crossflow. Linear flow... 111

Fig. 65-Gas/water flow in Zone 2 after foam forms in Zone 1. No Crossflow. Radial flow... 111

Fig. 66-Fronts in Zone 2 versus mobility ratio. Linear flow.......................................... 113

Fig. 67-Fronts in Zone 2 versus resistance factor in Zone 1. Linear flow.......................... 113

Fig. 68-Fronts in Zone 2 versus permeability contrast. Linear flow.................................... 114

Fig. 69-Fronts in Zone 2 versus gas-water mobility. Radial flow....................................... 115

Fig. 70-Fronts in Zone 2 versus resistance factor in Zone 1. Radial flow. ............................ 115

Fig. 71-Fronts in Zone 2 versus permeability contrast. Radial flow.................................. 116

Fig. 72-Fronts in Zone 2 for constant $F_{r} k_{2} / k_{1}$. Linear flow......................................... 117

Fig. 73-Trailing edges of the fingering region in Zone 2 for constant $F_{r} k_{2} / k_{1}$. Radial flow. 117

Fig. 74-Maximum allowable $F_{r 2} / F_{r 1}$ for linear flow, no crossflow.................................... 120

Fig. 75-Maximum allowable $F_{r 2} / F_{r l}$ for radial flow, no crossflow................................... 121

Fig. 76-Data from Vela et al. ${ }^{18}$ compared with maximum allowable $F_{r 2} / F_{r 1}$ values. ............ 121

Fig. 77-Data from Hirasaki and Pope ${ }^{20}$ compared with maximum allowable $F_{r 2} / F_{r 1}$ values.. 122

Fig. 78-Understanding front movements for linear flow with crossflow, moderate $F_{r}$ values 123

Fig. C-1 - Gas/water flow in Zone 2 after foam forms in Zone 1. No Crossflow. Linear flow . 145

Fig. C-2 - Gas/water flow in Zone 2 after foam forms in Zone 1. No Crossflow. Radial flow . 146 


\section{ACKNOWLEDGMENTS}

Financial support for this work is gratefully acknowledged from the United States Department of Energy (NETL/National Petroleum Technology Office), the State of New Mexico, ConocoPhillips, Marathon, and ExxonMobil. I greatly appreciate the efforts of those individuals who contributed to this project. Richard Schrader and Kate Wavrik performed the experimental work in Chapters 2 and 6, with help from Erica Ocampo, Depesh Patel, and Fred Frazier. Bob Sydansk was the primary author of Chapter 3. Ying Wang was primarily responsible for Chapter 4. Chapter 5 was dominantly the work of Dongmei Wang, with input from Peihui Han and Zhenbo Shao. Dongmei Wang and Peihui Han also provided important input for Chapter 6. Guoyin Zhang played a key role in Chapter 7. I especially appreciate the thorough review of this manuscript by Julie Ruff. 


\section{EXECUTIVE SUMMARY}

\section{Optimizing Disproportionate Permeability Reduction}

An idealistic goal of water shutoff technology is identifying materials that can be injected into any production well (without zone isolation) and will substantially reduce the water productivity without significantly impairing hydrocarbon productivity. Although many polymers and gels reduce permeability to water more than to oil or gas, several factors currently limit widespread field applications of this disproportionate permeability reduction property. First, adsorbed polymers and weak gels (suspensions of gel particles) show large variations in performance. Second, in unfractured wells (i.e., radial flow into porous sand or rock), the oil residual resistance factor, $F_{\text {rro }}$, (permeability reduction factor) must be reliably less than 2 . Third, adsorbed polymers and weak gels (suspensions of gel particles) reduce permeability by greater factors in low-permeability rock than high-permeability rock.

Pore-filling gels were investigated to overcome these limitations (Chapter 2). For porous media with pre-gel $k_{w}$ (at $S_{o r}$ ) ranging from 120 to $6,500 \mathrm{md}$, one $\mathrm{Cr}$ (III)-acetate-HPAM gel (with $0.5 \%$ HPAM) consistently reduced $k_{w}$ to about $0.24 \mathrm{md}$ (ranging from 0.12 to $0.37 \mathrm{md}$ ). In contrast, in Berea sandstone with $k_{w}$ (at $S_{o r}$ ) ranging from 222 to $363 \mathrm{md}$, a weak gel (with $0.18 \%$ polymer) exhibited a much wider range of post-polymer $k_{w}$ values - from 0.75 to $202 \mathrm{md}$. Thus, strong pore-filling gels can provide greater reliability and behavior that is insensitive to the initial rock permeability.

With sufficient oil throughput, pore-filling gels dehydrate, thus increasing permeability to oil. Several gel formulations provided water residual resistance factors greater than 2,000 and ultimate $F_{\text {rro }}$ values of 2 or less. These results provide hope that our current approach will identify gels that can successfully and reliably treat either fractured or unfractured production wells without zonal isolation. Significant oil throughput was required to achieve low $F_{\text {rro }}$ values, suggesting that gelant penetration into porous rock must be small (a few feet or less) for existing pore-filling gels to provide effective disproportionate permeability reduction.

\section{Guidelines for Relative Permeability Modification Treatments}

Chapter 3 provides guidance on where relative-permeabiliy-modification/disproportionatepermeability-reduction (RPM/DPR) treatments can be successfully applied for use in either oil or gas production wells. When properly designed and executed, these treatments can be successfully applied to a limited range of oilfield excessive-water-production problems. When these treatments are applicable, they may be placed using bullhead injection (not requiring mechanical zonal isolation) — a very favorable feature. However, there are a substantial number of limitations and possible pitfalls relating to the successful application of RPM/DPR treatments. First-time application by an inexperienced operator should be considered a somewhat high-risk undertaking. In order to successfully treat unfractured production wells (i.e., radial flow through matrix rock into the well) that are fully drawn down, the oil and water zones should not be in pressure communication and the oil-producing zone(s) must be producing at $100 \%$ oil cut (dry oil). When treating unfractured and multi-zoned production wells that are not fully drawn down, the well's long-term oil-production rate can be increased if the post-treatment drawdown is increased substantially. Treatments that promote short-term (transient) decreased water/oil ratios can, in principle, be applied to many unfractured production wells (that are not totally watered- 
out) in matrix rock reservoirs. However, these latter treatments must be custom designed and engineered on a well-by-well basis. Furthermore, for most wells, the performance and the economics of such transient treatments are generally marginal. An attractive application of $\mathrm{RPM} / \mathrm{DPR}$ treatments is the use of robust pore-filling gels in the matrix reservoir rock that is adjacent to a fracture(s) when oil and water is being co-produced into the treated fracture.

\section{Correlating Gel Rheology with Behavior during Extrusion}

In many successful conformance control treatments, large volumes of gels were extruded through fractures during placement. The pressure gradient for gel extrusion depends strongly on fracture width and gel composition. Extrusion experiments directly measure gel properties in fractures, but they are both expensive and time-consuming. In Chapter 4, we investigated whether using rheology measurements to assess gel properties in fractures might prove a good substitute for the extrusion experiments, at a much more reasonable cost. The rheology behavior of the gels tested showed a strong parallel to the results obtained from previous gel extrusion experiments. However, for a given aperture (fracture width or plate-plate separation), the pressure gradients measured during the gel extrusion experiments were much higher than anticipated from rheology measurements. Extensive experiments established that wall slip and first normal stress difference were not responsible for the pressure gradient discrepancy. Steady shear and oscillatory shear data were collected with a rheometer using both smooth and rough parallel-plate geometries and employing various gap heights. Wall-slip effects were present with smooth plates but negligible with rough plates.

To explain the discrepancy, we noted that the aperture for gel flow (for mobile gel wormholing through concentrated immobile gel within the fracture) was much narrower than the width of the fracture. Considering the shear-thinning gel properties, two models were developed using shell momentum balances. The first model explained why the pressure gradient for gel extrusion varied inversely with the square of the fracture width rather than inversely with fracture width. In particular, the relationship depends on the power-law index of the material. The second model correlated pressure gradient, shear stress, flow rate, and shear rate to bridge the gap between gel rheology in fractures versus in a rheometer.

\section{Sweep Improvement Options for the Daqing Oil Field}

Chapter 5 investigates the potential of various approaches for improving sweep in parts of the Daqing Oil Field that have been EOR targets. Possibilities included (1) gel treatments that are directed at channeling through fractures, (2) colloidal dispersion gels, (3) reduced polymer degradation, (4) more viscous polymer solutions, and (5) foams and other methods. Our studies indicated that the polymer flood should have provided excellent sweep throughout the vast majority of the patterns under consideration. However, since ASP flooding is being considered to increase recovery efficiency from the Daqing Oil Field, mobility control and sweep improvement will be especially important and challenging during implementation of any future ASP process.

Fractures were present in a number of Daqing wells (both injectors and producers). Because the fractures were narrow far from the wellbore, severe channeling did not occur. On the contrary, fractures near the wellbore aided reservoir sweep. These near-wellbore fractures substantially increased the injectivity index during injection of polymer solutions and increased oil productivity index in the production wells. These observations may be valuable during 
implementation of future floods where very low-mobility chemical (i.e., ASP) banks must be injected to maintain mobility control.

Several modes of polymer degradation were considered, with mechanical (shear) degradation being of most concern. Appropriate use of near-wellbore fractures may effectively mitigate mechanical degradation, as well as improving injectivity.

Several new polymers show potential for cost-effective improvements at Daqing. Increased polymer concentration was also considered. A number of other approaches are (or have been) under investigation, including colloidal dispersion gels, foams, ASP foams, steam, microbes, and polymer solutions prepared with reduced salinity.

\section{Are Colloidal Dispersion Gels Really a Viable Technology?}

In the February 2006 issue of the Journal of Petroleum Technology, a "Distinguished-AuthorSeries" paper claimed that a process using aqueous colloidal dispersion gels (CDG gels) performed superior to polymer flooding. Unfortunately, this claim is misleading and generally incorrect (Chapter 6). Colloidal dispersion gels, in their present state of technological development, should not be advocated as an improvement to, or substitute for, polymer flooding. Laboratory results published by three independent universities confirm that CDG gelants and gels behave like other gels used in conformance control. In particular, before gelation (or before significant crosslinking occurs), gelants show the same flow properties in porous media as polymer solutions without crosslinker. After crosslinking, the gels do not flow through porous rock. Gelation times for CDG gels are fairly short_less than one day even at low temperatures. Shearing CDG gelants through porous rock at high velocities may delay gelation and development of high resistance factors by a few hours, but certainly NOT for days, weeks, or months, as speculated by the CDG vendor. One-day-old colloidal dispersion gels do not flow through porous media with permeabilities less than 8 darcys.

We have yet to see a convincing case that supports the untenable claims made by the CDG vendor and their representative (i.e., that CDG gels act as super polymer solutions and that CDG gels preferentially enter high-permeability strata and later divert water into low-permeability zones). Instead, credible explanations can be envisioned that are consistent both with field and laboratory findings. Careful, objective analysis of the Daqing CDG field data suggest no credible, unambiguous improvement of the CDG flood over the normal polymer flood. Injectivity behavior was not significantly different for the two cases. Also, water/oil ratios and production trends could not be credibly or unambiguously distinguished for the two processes. These observations were consistent with laboratory results indicating that the aluminum was either removed (by adsorption or precipitation) or was present in concentrations too low to allow polymer crosslinking within the reservoir.

\section{Can Foams Provide Superior Mobility Control Compared with Polymers?}

We investigated when foams could be superior to polymers for improving sweep efficiency in reservoirs (Chapter 7). Our focus was on sweep improvement during waterflooding and chemical flooding rather than gas flooding. Use of foams could provide superior vertical sweep compared to polymer solutions if (1) foam forms in the high-permeability zone(s) but not in the lowpermeability zone(s), (2) no crossflow occurs between the high- and low-permeability zones, 
AND (3) the foam resistance factor in the high-permeability zone(s) is sufficiently high to overcome the permeability contrast and the unfavorable mobility ratio between the gas bank and the oil/water bank in the less-permeable zones. Foams will generally not be superior to polymers under other circumstances (unless gravity effects provide a fortuitous benefit).

Other limitations associated with foam use must also be recognized, including (1) challenges with formulating foams for individual reservoirs to meet the above requirements, (2) limitations on foam propagation, especially due to surfactant retention, (3) compression costs associated with foam injection, and (4) limitations on foam stability under reservoir conditions.

\section{How is Vertical Sweep Affected When Resistance Factors Vary with Permeability?}

Historically, permeability reduction by polymers was advocated as advantageous since reduction in polymer mobility was greater than anticipated based on solution viscosity. However, this permeability reduction generally increases with decreasing permeability, potentially diminishing sweep efficiency. Chapter 8 examined vertical sweep efficiency as a function of (1) permeability contrast, $k_{1} / k_{2}$, (2) presence or absence of crossflow, and (3) ratio of resistance factors in neighboring zones, $F_{r 2} / F_{r l}$. (Subscript 1 applies to the high-permeability zone, while Subscript to refers to the low-permeability zone).

For applications with linear flow (e.g., fractured wells) when crossflow cannot occur, the maximum allowable ratio of $F_{r 2} / F_{r 1}$ (so that polymer injection does not harm vertical sweep) is about the same as the permeability ratio, $k_{1} / k_{2}$. Thus, linear flow applications can be reasonably forgiving if the permeability contrast and the polymer solution resistance factors are sufficiently large. Radial flow is much less forgiving to high values of $F_{r 2} / F_{r l}$. Even for high permeability contrasts (e.g., $k_{1} / k_{2}=20$ ), the maximum allowable $F_{r 2} / F_{r 1}$ values were less than 1.4.

In most cases when crossflow can occur, the $F_{r 2} / F_{r 1}$ ratio has little effect on the relative distance of polymer penetration into the various zones. 


\section{INTRODUCTION}

Fractures, vugs, karst, and similar void channels often cause excess water production and poor sweep efficiency in reservoirs. In both hydraulically and naturally fractured reservoirs, void channels often allow injected fluids to flow directly between injection and production wells. This problem is especially important for enhanced oil recovery projects, where high-value fluids are injected. In production wells, void channels often extend into an aquifer - thus accentuating water production. In many cases, gels have effectively mitigated channeling through fractures, fracture-like features, and voids. Gels have reduced channeling through fractures in waterfloods and gas floods. Gels have also reduced water production in wells where fractures, fracture-like features, and voids connect to an underlying aquifer. Although many gel treatments have been quite successful, many important questions exist concerning how best to design and implement them. Current methods are very sensitive to the aperture of the fracture or void. Unfortunately, these apertures are usually not known in field applications. Thus, a particular need exists for treatments that are not sensitive to the aperture of the fracture or void.

\section{Objectives}

The project objective is to develop aperture-tolerant, chemical-based methods to reduce channeling through voids (e.g., fractures, vugs, karst) during hydrocarbon production. The project has two tasks. The first task develops materials that can be effectively placed and will consistently minimize flow through voids with a wide range of apertures. The second task develops methods to minimize water entry into voids from the surrounding rock. This task requires chemicals (i.e., gels, polymers) that predictably and controllably reduce the permeability to water much more than that to hydrocarbon.

\section{Report Content}

This report describes work performed during the project. Chapter 2 describes a promising method to maximize disproportionate permeability reduction using pore-filling gels. Chapter 3 provides guidance on where relative-permeabiliy-modification/disproportionate-permeabilityreduction treatments can be successfully applied for use in either oil or gas production wells. Chapter 4 examines whether gel rheology can explain behavior during extrusion through fractures. Chapter 5 considers sweep improvement options for the Daqing Oil Field. Chapter 6 describes an investigation of the viability of colloidal dispersion gels. Chapter 7 investigates when foams could be superior to polymers for improving sweep efficiency in reservoirs. Chapter 8 examines how vertical sweep is affected when resistance factors vary with permeability. Finally, technology transfer activities are described in Appendix A. 


\section{OPTIMIZING DISPROPORTIONATE PERMEABILITY REDUCTION}

\section{Introduction}

Many polymers and gels can reduce permeability to water more than that to oil or gas. ${ }^{1-5}$ This disproportionate permeability reduction (or "relative permeability modification") is essential if polymers or gelants are placed in production wells without protecting hydrocarbon-productive zones. ${ }^{6}$ With existing polymers, gels, and technology, disproportionate permeability reduction may have its greatest value when treating production wells that intersect a fracture or fracturelike features. ${ }^{7-9}$ Nonetheless, many people are very interested in exploiting this property to reduce excess water production from unfractured wells (i.e., radial flow into porous rock or sand). The idealistic goal of this technology is to develop a material that can be injected into any production well (without zone isolation) and will substantially reduce water productivity index without significantly impairing hydrocarbon productivity. Several obstacles must be overcome before this ideal can be achieved.

\section{Challenges for Applications of Disproportionate Permeability Reduction}

Variable Performance. Field applications of polymer and gel treatments have shown substantial variations in performance from one application to the next. In part, these variations arise from differences in reservoir conditions, well conditions, and mixing and injection procedures. However, significant performance variations appear inherent for some polymers and gels. ${ }^{10,11}$ During replicate experiments (in Berea sandstone) with a commercially available weak gel, oil residual resistance factors ( $F_{\text {rro }}$, permeability reduction factors) ranged from 2.7 to 59 (median of 5.9 , average of 9.7, and standard deviation of 13.5), while water residual resistance factors $\left(F_{r r w}\right)$ ranged from 1.5 to 317 (median of 6.6, average of 32, and standard deviation of 78). ${ }^{10}$

Uncontrolled variability of residual resistance factors may be an inherent flaw for adsorbed polymers and weak gels. Permeability reduction by adsorbed polymers can be strongly influenced by mineralogy of the rock. In turn, rock mineralogy typically exhibits significant variations locally within a porous medium. Consequently, these mineralogical variations could lead to wide differences in performance for adsorbing polymers.

Weak gels are typically suspensions of gel particles-not a continuous three-dimensional gel structiure. ${ }^{12-15}$ These suspensions have a particle size distribution-they are not monodisperse. Pores within a rock also have a size distribution. Since the particles reduce permeability by lodging in pore throats, the ratio of particle size to pore throat size is important in determining residual resistance factors for these suspensions. Variations in particle size distribution (especially resulting from unknown or uncontrolled particle generation) and variations in throat size distribution (resulting from normal geologic processes) may cause wide variations in performance for particle suspensions. An extensive effort is underway at Institut Francais du Petrole (IFP) to address this issue. IFP is developing suspensions of "microgels" that are manufactured with very narrow particle size distributions. ${ }^{16}$

$F_{\text {rro }}$ Must Be $<2$ for Radial Flow. A second challenge is presented by the requirements for successful application of disproportionate permeability reduction for different types of problems. For example, consider an unfractured (i.e., radial flow) production well with one water zone, one oil zone, and a separating impermeable shale barrier. Fig. 1 illustrates that productivity losses in 
radial flow are much more sensitive to residual resistance factors than to radius of gel penetration $\left(r_{g e l}\right)$. To avoid excessive losses in oil productivity when gelant is placed using unrestricted injection (i.e., no zone isolation), the gel must provide a residual resistance factor less than 2 in the oil zone. ${ }^{6,13,17}$ Preferably, the gel should provide a residual resistance factor greater than 20 in the water zone (Fig. 1). The variations in residual resistance factors mentioned above point to the difficulties in reliably attaining permeability reductions to oil that are less than two.

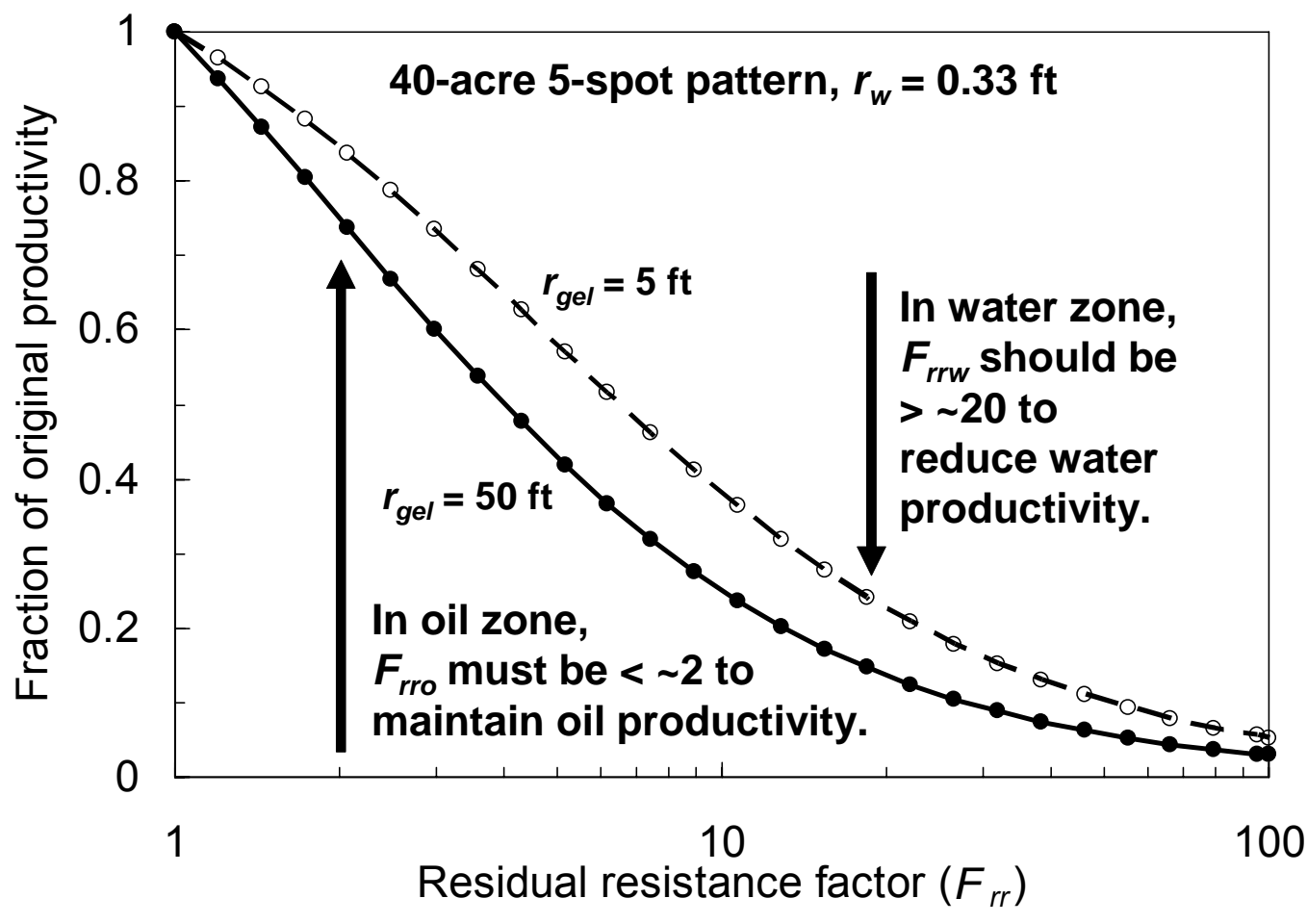

Fig. 1-Losses of zone flow capacity for radial flow.

Permeability Dependence of $\boldsymbol{F}_{r r}$. A third challenge comes from the dependence of residual resistance factors on the permeability of the porous media. For adsorbing polymers and weak gels, resistance factors and residual resistance factors increased with decreased permeability. ${ }^{14,15,18-21}$ In other words, these materials damage low-permeability rock more than high-permeability rock. Depending on the magnitude of this effect, these polymers and gels can harm injection or production flow profiles in wells, even though the polymer or gelant penetrates significantly farther into the high-permeability rock. ${ }^{6,13,17}$

\section{Overcoming the Obstacles}

Variability. Variability of residual resistance factors was the first challenge mentioned above. This variability can be attributed to uncontrolled particle size distributions for suspensions of gel particles and to mineralogical variations for both adsorbed polymers and suspensions of gel particles. Perhaps this variability in performance can be mitigated by using a permeability reduction mechanism with better control. In particular, if all aqueous pore space was filled with a uniform gel, the permeability reduction (at least for water flow) would be controlled by flow through the gel itself. If the inherent permeability of the gel to water was much less than the 
permeability of the original porous media, the permeability reduction would not be sensitive to variations in mineralogy, pore size, or pore size distribution.

Concerning variability of oil residual resistance factors, our recent work ${ }^{22}$ suggested that reestablishing oil permeability in a gel-filled porous media can be predicted using concepts of mobility ratios and stable-versus-unstable displacements.

Linear versus Radial Flow. The second hurdle mentioned depended on the type of problem to be treated. Our work suggests that disproportionate permeability reduction currently has its greatest utility in treating fractures and fracture-like features. ${ }^{7,8,9}$ If gelant is allowed to leakoff a short, controlled distance from the fracture faces and if the gel provides predictable residual resistance factors, water entry into the fracture can be greatly impeded while causing minimal reduction in hydrocarbon productivity. This process does not require that the gel provide very low oil residual resistance factors - only that the gel provides water residual resistance factors that are reliably much greater than oil residual resistance factors (see Fig. 2 for an example).

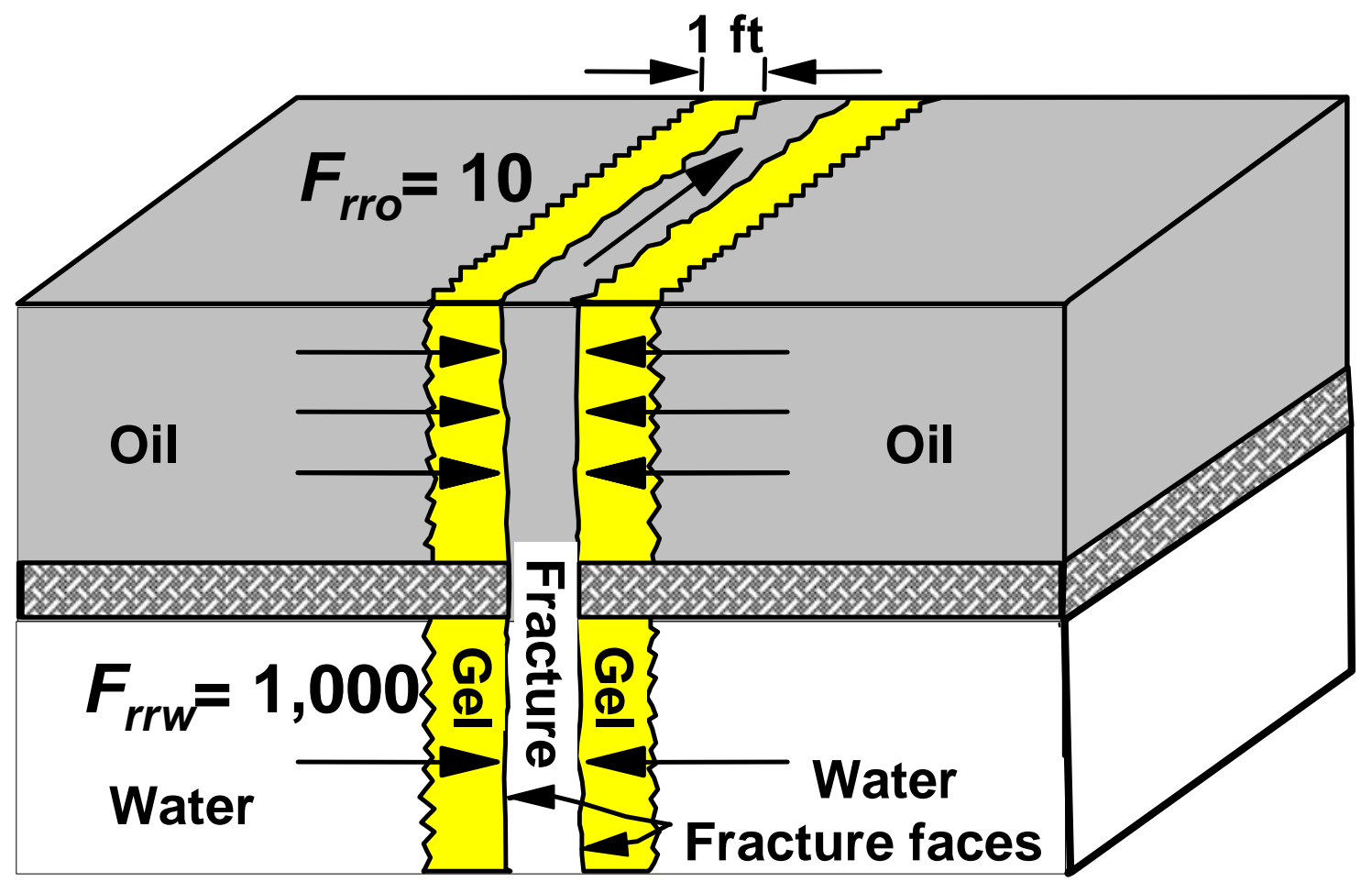

Equivalent resistance (expressed as distance through untreated rock) that fluid must flow to traverse the gel bank and enter the fracture:

In oil zone: $1 \mathrm{ft} \times 10=10 \mathrm{ft}$.

In water zone: $1 \mathrm{ft} \times 1,000=1,000 \mathrm{ft}$.

Fig. 2-Gel restricting water entry into a fracture. 
In contrast, for radial flow from wells into porous rock (i.e., unfractured production wells), the oil residual resistance factor $\left(F_{\text {rro }}\right)$ must generally have a value below $2^{6,13}$ (see Fig. 1). Normally, we might not expect this to be achievable using a pore-filling gel, such as $\mathrm{Cr}$ (III)acetate-HPAM. We typically expect pore-filling gels to provide high residual resistance factors for both oil and water. However, our recent work provides hope that low $F_{\text {rro }}$ values may be attained. $^{22}$ For example, in one case before gel placement, a Berea core showed an endpoint permeability to oil of $508 \mathrm{md}$ (i.e., at $S_{w r}$ ) and an endpoint permeability to water of $120 \mathrm{md}$ (i.e., at $S_{\text {or }}$ ). After placement of a Cr(III)-acetate-HPAM gel [with $0.5 \%$ HPAM and $0.0417 \% \mathrm{Cr}$ (III) acetate], the permeability during brine injection quickly stabilized at $0.17 \mathrm{md}$ (open circles in Fig. 3) -indicating a water residual resistance factor of 706 (i.e., 120/0.17). In contrast, during oil (hexadecane) injection after gel placement (solid circles in Fig. 3), the permeability rose gradually to $105 \mathrm{md}$ over the course of 100 pore volumes $(P V)$-indicating an oil residual resistance factor of only 4.8 (i.e., 508/105). Since the permeability to oil was still rising at 100 $P V$ (Fig. 3), hope exists that even lower oil residual resistance factors could be achieved.

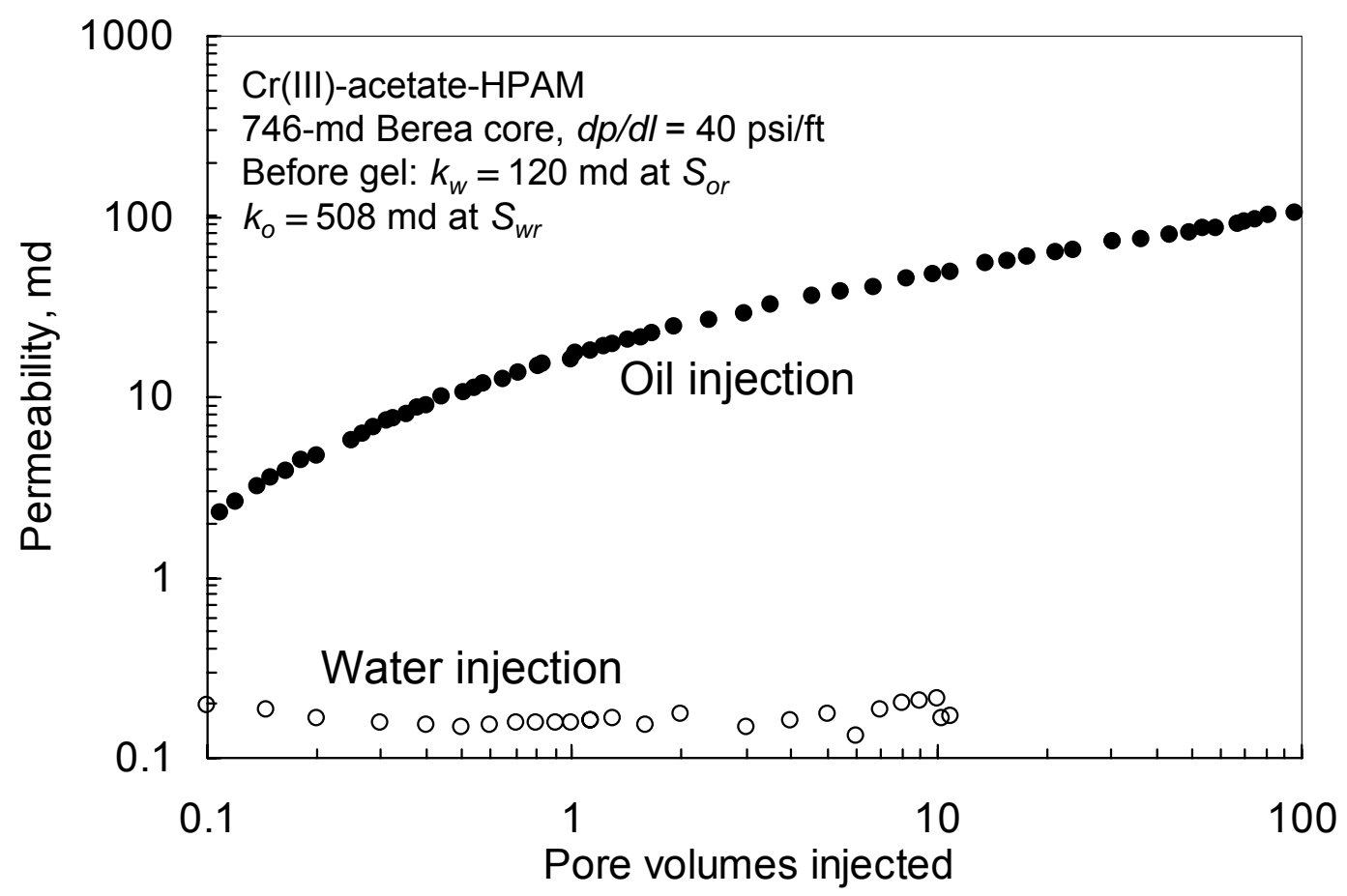

Fig. 3-Permeabilities to oil and water after gel placement.

Permeability Dependence. The third challenge mentioned above was the permeability dependence of residual resistance factors. For adsorbing polymers and suspensions of gel particles, residual resistance factors increased with decreased permeability. In contrast, porefilling gels reduced the permeability to water of all porous media to the same low value - a value that approximates the inherent permeability of the gel to water. ${ }^{12-15}$ Consequently, use of porefilling gels may provide a means to overcome some of the important challenges that have limited applications of disproportionate permeability reduction. 


\section{Permeability to Water after Gel Placement}

$\boldsymbol{k}_{w}$ versus Initial Core Permeability and Core Material. With the above considerations in mind, we performed several experiments in an effort to use pore-filling Cr(III)-acetate-HPAM gels to optimize disproportionate permeability reduction. One goal was to ensure that water residual resistance factors $\left(F_{r r w}\right)$ are reliably high. For radial flow, Fig. 1 suggests that $F_{r r w}$ values should be greater than 20. For linear flow applications (e.g., fractured wells), much larger $F_{r r w}$ values are desirable (see Fig. 2). Thus, we performed experiments to establish whether a porefilling gel can provide reliable $k_{w}$ and $F_{r r w}$ values. In particular, for the first set of experiments (all at $41^{\circ} \mathrm{C}$ ), we wished to confirm that a pore-filling gel reduces the permeability $\left(k_{w}\right)$ of all porous media to the same low value - that reflects the inherent permeability of the gel to water. In Table 1, the first six entries describe experiments where cores at residual oil (hexadecane) saturation $\left(S_{o r}\right)$ were flooded with a $\mathrm{Cr}(\mathrm{III})$-acetate-HPAM gelant [with $0.5 \%$ Ciba Alcoflood 935 ${ }^{\mathrm{TM}}$ HPAM, $0.0417 \% \mathrm{Cr}(\mathrm{III})$ acetate, $1 \% \mathrm{NaCl}, 0.1 \% \mathrm{CaCl}_{2}$ ], shut in for 3 days to allow gelation, followed by brine injection $\left(1 \% \mathrm{NaCl}, 0.1 \% \mathrm{CaCl}_{2}\right)$ at a fixed pressure gradient. Initial core permeabilities ranged from 746 to 15,270 md in core materials including Berea sandstone, fused silica, and porous polyethylene. At $S_{o r}, k_{w}$ values before gel placement ranged from 120 to $6,500 \mathrm{md}$. For the first six post-gel cases, $k_{w}$ at $S_{\text {or }}$ averaged $0.24 \mathrm{md}( \pm 0.084 \mathrm{md}$ ), and no trend was evident when correlating with material type or initial core permeability (solid circles in Fig. 4). These results support our previous finding that pore-filling gels reduce permeability of all porous media to a value that reflects the inherent permeability of the gel to water. ${ }^{12,14,15}$ In contrast, in Berea sandstone with $k_{w}$ (at $S_{o r}$ ) ranging from 222 to $363 \mathrm{md}$, a weak gel (with $0.18 \%$ polymer) exhibited a much wider range of post-polymer $k_{w}$ values-from 0.75 to 202 md (open circles in Fig. 4, data from Ref. 10). Thus, pore-filling gels can provide greater reliability and behavior that is insensitive to the initial rock permeability.

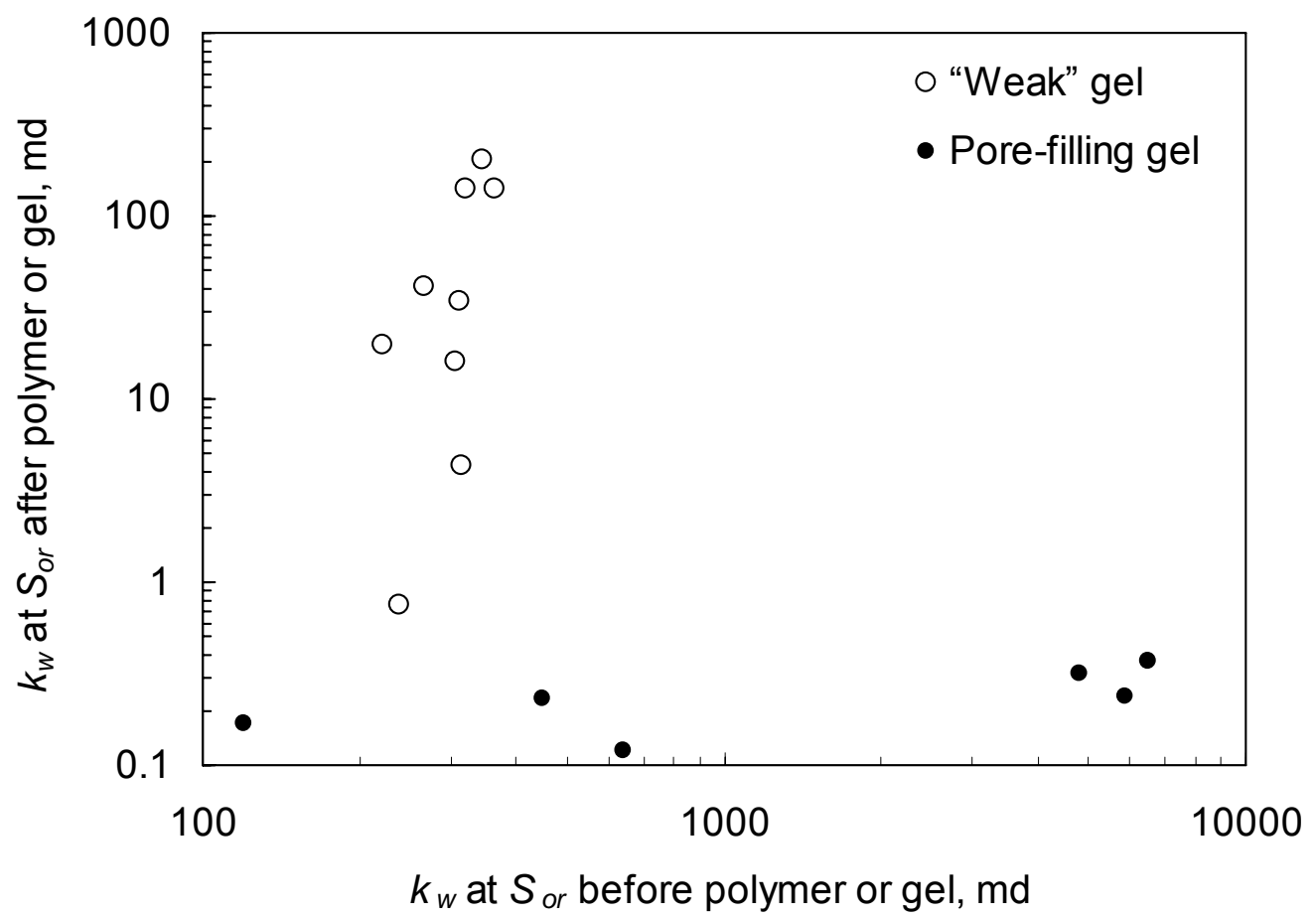

Fig. 4-Variations in $k_{w}$ for pore-filling gels versus weak gels. 
Table $1-k_{w}$ during brine flow after gel placement.

\begin{tabular}{|c|c|c|c|c|c|c|c|c|c|}
\hline Entry & $\begin{array}{c}\text { Core } \\
\text { material }\end{array}$ & $\begin{array}{c}\text { Initial } \\
k, \\
\text { md }\end{array}$ & $\begin{array}{c}\phi, \\
\%\end{array}$ & $\begin{array}{c}k_{w} \text { at } \\
S_{\text {or }}, \\
\text { md }\end{array}$ & $\begin{array}{c}\text { HPAM } \\
\text { in gel, } \\
\%\end{array}$ & $\begin{array}{c}S_{\text {or }} \\
\text { present? }\end{array}$ & $\begin{array}{c}d p / d l, \\
\text { psi/ft }\end{array}$ & $\begin{array}{c}\text { Post- } \\
\text { gel } k_{w} \\
\text { md }\end{array}$ & $F_{r r w}$ \\
\hline 1 & Berea & 746 & 21 & 120 & 0.5 & yes & 40 & 0.17 & 706 \\
\hline 2 & fused silica & 1,820 & 27 & 447 & 0.5 & yes & 30 & 0.23 & 1,940 \\
\hline 3 & fused silica & 2,390 & 27 & 640 & 0.5 & yes & 10 & 0.12 & 5,330 \\
\hline 4 & polyethylene & 6,400 & 40 & 4,810 & 0.5 & yes & 100 & 0.32 & 15,000 \\
\hline 5 & polyethylene & 9,530 & 40 & 5,860 & 0.5 & yes & 30 & 0.24 & 24,400 \\
\hline 6 & polyethylene & 15,270 & 40 & 6,500 & 0.5 & yes & 10 & 0.37 & 17,600 \\
\hline 7 & Berea & 356 & 21 & $*$ & 0.5 & no & 13.7 & 0.015 & 23,700 \\
\hline 8 & Berea & 389 & 21 & $*$ & 0.5 & no & 30 & 0.005 & 77,800 \\
\hline 9 & Berea & 100 & 21 & $*$ & 0.5 & no & 58 & 0.01 & 10,000 \\
\hline 10 & Berea & 40 & 21 & $*$ & 0.4 & no & 58 & 0.019 & 2,110 \\
\hline 11 & Berea & 274 & 21 & $*$ & 0.3 & no & 58 & 0.055 & 4,980 \\
\hline 12 & Berea & 98 & 21 & 31 & 0.5 & yes & 58 & 0.007 & 4,430 \\
\hline 13 & Berea & 69 & 21 & 14.4 & 0.3 & yes & 58 & 0.092 & 157 \\
\hline 14 & polyethylene & 8,100 & 40 & $*$ & 0.5 & no & 30 & 0.06 & 135,000 \\
\hline 15 & sand pack & 8,100 & 35 & $*$ & 0.5 & no & 1.5 & 0.029 & 279,000 \\
\hline 16 & polyethylene & 2,000 & 40 & $*$ & 0.5 & no & 24.5 & 0.14 & 14,300 \\
\hline 17 & polyethylene & 3,200 & 40 & $*$ & $0.125 \dagger$ & no & 24.5 & 0.23 & 13,900 \\
\hline
\end{tabular}

* Since no residual oil was present, $F_{r r w}$ was calculated using the initial core permeability. † xanthan instead of HPAM.

$\boldsymbol{k}_{w}$ with/without $\boldsymbol{S}_{\text {or }}$ Entries 7-9 and 14-16 in Table 1 list results for the same gel (0.5\% HPAM) but in porous media with no initial oil saturation (i.e., the cores were completely saturated with gel before brine was injected). For these six cases, a larger degree of variation was seen (average post-gel $k_{w}$ was $0.043 \mathrm{md} \pm 0.047 \mathrm{md}$ ) compared to that for the first six entries. Even so, most measured post-gel $k_{w}$ values with no $S_{o r}$ were noticeably lower than those cases with a residual oil saturation. Why should permeability to water be higher when residual oil is present? Several possibilities come to mind. First, brine could breach or fracture through the gel. With residual oil drops dispersed throughout the porous medium, breaking pathways through gel films (that separate oil drops) might be easier than breaking a path through one continuous block of gel. However, such a breaking mechanism should depend on the pore size: gel breaching should be easier in large pores and very permeable media than in small pores and low-permeability rock. Generally, we did not see a significant trend for post-gel $k_{w}$ values as initial core permeability increased (Entries 1-6 in Table 1). However, Entry 12 (with initial $k$ of $98 \mathrm{md}$ and $k_{w}=31 \mathrm{md}$ at $\left.S_{o r}\right)$ showed a post-gel $k_{w}$ value $(0.007 \mathrm{md})$ that was much less than those for Entries 1-6. Interestingly, post-gel $k_{w}$ values for Entries 9 and $12(0.01$ versus $0.007 \mathrm{md})$ suggest no significant effect of an initial residual oil saturation for this set of cases.

A second conceivable mechanism is that brine could force a pathway between the gel and the walls of the porous media. However, for this mechanism, the ability to form a pathway (i.e., 
higher $k_{w}$ values) should be enhanced with (1) increased initial permeability (i.e., decreased rockgel surface area) and (2) increased hydrophobic nature of the rock surface. In support of this mechanism, the post-gel $k_{w}$ values for the polyethylene cores (Entries 14 and 16) were noticeably greater than those for the Berea cores (Entries 7 to 9).

The remaining mechanism is that water dominantly forces a pathway between the gel and the residual oil. Additional work is needed to clarify the importance of these mechanisms.

$\boldsymbol{k}_{w}$ versus Polymer Content. In previous work with $\mathrm{Cr}(\mathrm{III})$-acetate-HPAM gels, ${ }^{23,24}$ results suggested that the inherent permeability of the gel to water ( $k_{\text {gel }}$ in $\mathrm{md}$ ) varied inversely with the third power of polymer concentration $(C$ in \%).

$$
k_{\text {gel }}=0.125 / C^{3} .
$$

Part of our approach to optimizing disproportionate permeability reduction involves controlling the inherent permeability of the gel to water. So, we examined the performance of gels as a function of polymer content.

Entries 9 through 11 examines three Berea cores that were saturated with three different compositions of $\mathrm{Cr}$ (III)-acetate-HPAM gel, with HPAM concentrations of $0.5 \%, 0.4 \%$, and $0.3 \%$, respectively. The cores had the same dimensions and were cut from the same slab of Berea sandstone, but interestingly, the initial rock permeabilities (before gel placement) varied from 40 to $274 \mathrm{md}$. We should mention one "trick" to ensure that pore-filling $\mathrm{Cr}(\mathrm{III})$-acetate-HPAM gels form in Berea sandstone. That trick involved flushing the cores with a few pore volumes of brine containing $0.12 \% \mathrm{Cr}(\mathrm{III})$ acetate before injecting the gelant. This process saturates chromium adsorption sites and minimizes depletion of chromium from the gelant during placement. Prior to using this procedure, we often had problems with incomplete gel formation in Berea sandstone.

During brine flow (at $58 \mathrm{psi} / \mathrm{ft}$ ) after gelation, post-gel $k_{w}$ values were $0.01 \mathrm{md}, 0.019 \mathrm{md}$, and $0.055 \mathrm{md}$, respectively. For the gel with $0.5 \%$ HPAM, the 0.01 -md value (Entry 9) was similar to the 0.015 -md and $0.005-\mathrm{md}$ values associated with Entries 7 and 8 -indicating a reasonable degree of reproducibility for the results. However, these values were substantially lower than the 1-md value predicted from Eq. 1. This discrepancy may be tied to differences in porosity of the porous media. In particular, Eq. 1 was based on flow through solid pieces of gel-i.e., 100\% porosity with no rock..$^{23,24}$

On the other hand, if we accept the 0.01-md value for the gel with $0.5 \%$ HPAM, the post-gel $k_{w}$ values for Entries 10 and 11 were consistent with the cubic relation of Eq. 1 between polymer concentration and inherent gel permeability. In particular, for Entries 9-11, post-gel $k_{w}$ values of $0.01 \mathrm{md}, 0.019 \mathrm{md}$, and $0.055 \mathrm{md}$ were observed, while Eq. 1 predicted values of $0.01 \mathrm{md}, 0.019$ $\mathrm{md}$, and $0.046 \mathrm{md}$ (if $0.01 \mathrm{md}$ was accepted as correct for the $0.5 \%$-HPAM case).

Entries 12 and 13 in Table 1 provide another set for comparison, using low-permeability Berea sandstone cores with residual oil present before gelant placement. Here, the gel with $0.3 \%$ HPAM (Entry 13) exhibited a post-gel $k_{w}$ value that was 13 times that for the gel with $0.5 \%$ HPAM (Entry 12). 
For yet another comparison, Entry 17 in Table 1 lists results for a gel that contained $0.125 \%$ xanthan (Kelco Kelzan $\mathrm{XCD}^{\mathrm{TM}}$ ), $0.0125 \% \mathrm{Cr}(\mathrm{III})$ acetate, $1 \% \mathrm{NaCl}$, and $0.1 \% \mathrm{CaCl}_{2}$. Before adding crosslinker, the xanthan polymer solution was clarified by filtration to remove cellular debris. Use of xanthan allowed formation of a pore-filling gel with a considerably lower polymer concentration than when using HPAM. Presumably, the semi-rigid-rod structure of xanthan allows the critical overlap concentration to be much lower than that for the random-coil structure of HPAM in solution.

The Cr(III)-acetate-xanthan gelant was placed in a 3.2-darcy polyethylene core (no residual oil present), and a brine pressure gradient of $24.5 \mathrm{psi} / \mathrm{ft}$ was applied $\left(41^{\circ} \mathrm{C}\right)$. During brine injection, the post-gel $k_{w}$ quickly stabilized at $0.23 \mathrm{md}$. This value can be compared with 0.06 md for Entry 14 or with 0.14 for Entry 16 (which used gels with $0.5 \%$ HPAM in porous polyethylene). Since the polymer concentration for Entry 17 was four times less than those for Entries 14 and 16, the post-gel $k_{w}$ for Entry 17 might be expected to be 64 times (i.e., $4^{3}$ ) greater than those for Entries 14 or 16 -i.e., $3.8 \mathrm{md}(0.06 \mathrm{md} \times 64)$ or $9.0 \mathrm{md}(0.14 \mathrm{md} \times 64)$ instead of $0.23 \mathrm{md}$. Alternatively, Entry 17 can be compared with Entry 11, where the post-gel $k_{w}$ was 0.055 md for a gel with $0.3 \%$ HPAM in Berea sandstone. For this case, since the polymer concentration for Entry 17 was 2.4 times less than that for Entry 11, the post-gel $k_{w}$ for Entry 17 might be expected to be 13.8 times (i.e., $\left.2.4^{3}\right)$ greater than that for Entry 11 -i.e., a value of $0.76 \mathrm{md}(0.055 \mathrm{md} \mathrm{x}$ 13.8) instead of $0.23 \mathrm{md}$. Thus, for both comparisons, the post-gel $k_{w}$ for the $\mathrm{Cr}$ (III)-acetatexanthan gel was significantly less than expected from the behavior of $\mathrm{Cr}$ (III)-acetate-HPAM gels. This result reveals limitations in using Eq. 1 for estimating post-gel $k_{w}$ values. Nevertheless, the post-gel $k_{w}$ value for Entry 17 was the highest for any pore-filling gel examined to date for cases where residual oil was not present during gelant placement.

Stability of Post-Gel $\boldsymbol{k}_{w}$. In the next section, we show that permeability to oil $\left(k_{o}\right)$ after gel placement was a strong function of time and throughput. In contrast, if the gel was not compromised (e.g., by exposure to high pressure gradients), Fig. 5 demonstrates that the post-gel $k_{w}$ was stable for a substantial period. In particular, for the gel associated with Entry 14 in Table $1, k_{w}$ held a value of about $60 \mu \mathrm{d}(0.06 \mathrm{md})$ for over 500 days during continuous exposure to a pressure gradient of $30 \mathrm{psi} / \mathrm{ft}$ at $41^{\circ} \mathrm{C}$. At 530 days, the permeability jumped from 60 to $80 \mu \mathrm{d}$, for unexplained reasons. Since then, the permeability has been stable.

Are $\boldsymbol{F}_{\boldsymbol{r r w}}$ Values High Enough? For radial flow, the residual resistance factor in the water zone should be at least 20 (Fig. 1). Will the results from Table 1 meet this requirement? The highest post-gel $k_{w}$ value listed was $0.37 \mathrm{md}$. Given the definition of water residual resistance factor (i.e., permeability to water before gel placement divided by permeability to water after gel placement), $F_{r r w}$ will be $\geq 20$ if the permeability to water before gel placement is greater than 7.4 md (i.e., 20x0.37). Many engineers and geologists (including the author) believe that if a productive oil reservoir has a matrix permeability below $20 \mathrm{md}$, fractures or fracture-like features probably play a major role in allowing fluid to flow to the wells. Consequently, these gels would provide acceptable $F_{r r w}$ values for radial flow applications. 


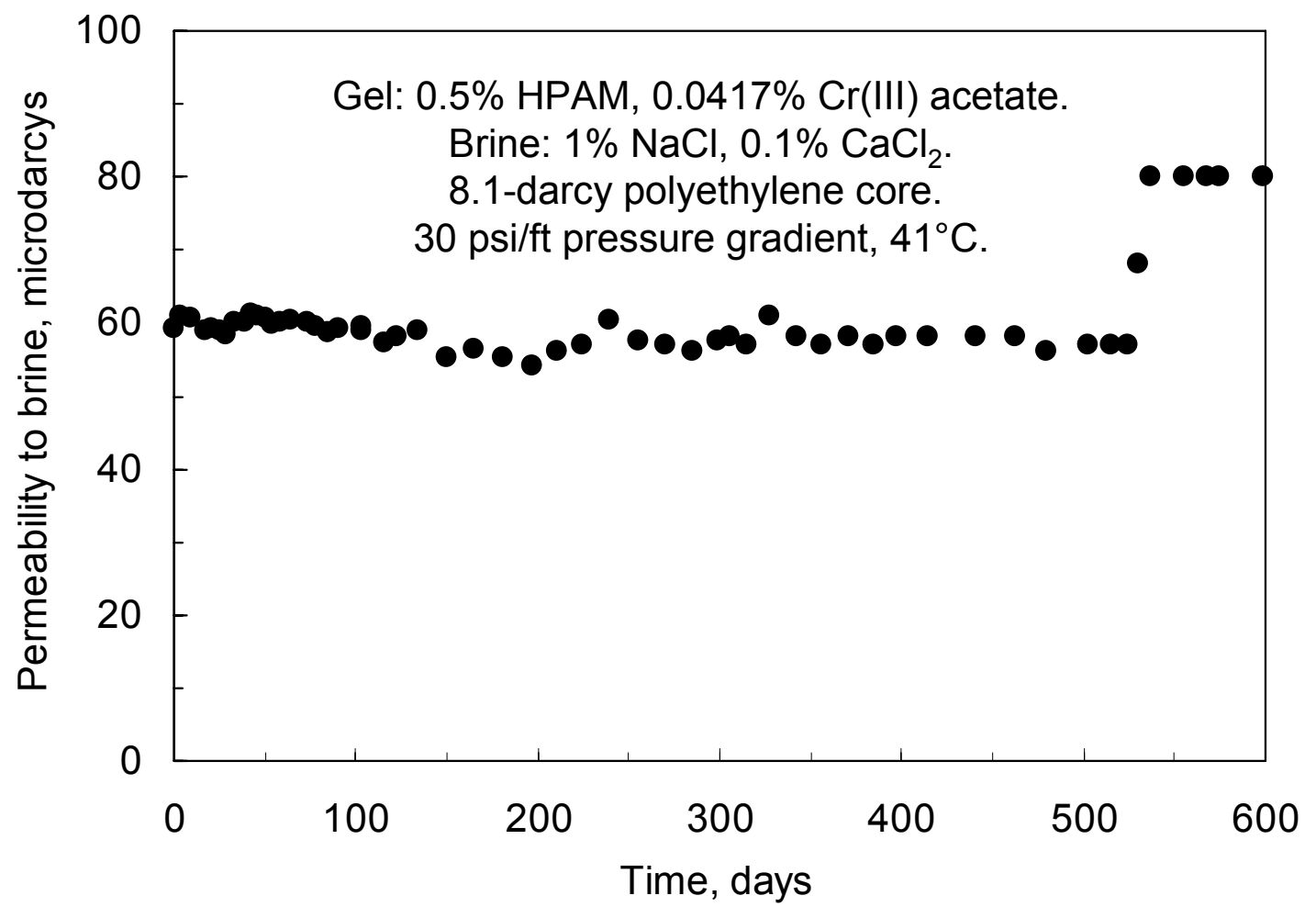

Fig. $5-k_{w}$ versus time after gel placement.

When treating fractures using the concept illustrated in Fig. 2, will the post-gel $k_{w}$ values from Table 1 be satisfactory? The degree of productivity impairment $\left(q / q_{o}\right)$ for flow into a fracture can be estimated using Eq. 2:

$$
q / q_{o} \approx L_{e} /\left(F_{r r} L_{p}+L_{e}\right),
$$

where $L_{p}$ is the distance of polymer or gelant leakoff from the fracture face and $L_{e}$ is the effective external drainage distance. From Eq. 2, reducing water productivity index by at least $50 \%$ requires that $F_{r r w} L_{p} \geq L_{e}$. If $L_{e}$ is $100 \mathrm{ft}, F_{r r w}$ must be at least 10 if $L_{p}$ is $10 \mathrm{ft}$ and at least 100 if $L_{p}$ is $1 \mathrm{ft}$. If $k_{w}$ after gel placement is $0.37 \mathrm{md}$ (Table 1), a $F_{r r w}$ value of at least 10 can be achieved if $k_{w}$ before gel placement is at least $3.7 \mathrm{md}$ (i.e., $10 \mathrm{x} 0.37 \mathrm{md}$ ). A $F_{r r w}$ value of at least $100 \mathrm{can}$ be attained if $k_{w}$ before gel placement is at least $37 \mathrm{md}$ (i.e., $100 \times 0.37 \mathrm{md}$ ).

These calculations reveal that gels can achieve beneficial reductions in water productivity for both linear and radial flow problems. However, they will not be effective in all situations. Effective applications require attention to ensure that the distance of gelant penetration is adequate for rock of a given permeability. For example, many West TX fractured dolomite reservoirs have rock permeability around $10 \mathrm{md}$. If a gel provides a $k_{w}$ after gel placement of $0.24 \mathrm{md}$ (average of Entries 1-6 in Table 1), a fairly large gelant leakoff distance may be needed (i.e., $>2.4 \mathrm{ft}$ ) to reduce water productivity by more than $50 \%$. Alternatively, if a gel provides a $k_{w}$ after gel placement of 0.007 md (Entry 12 in Table 1), a much smaller gelant leakoff distance (i.e., $0.07 \mathrm{ft}$ ) may provide the same effect. Large gelant leakoff distances present challengesespecially with respect to penetration of high molecular weight polymers into tight rock. 
Different gel formulations-e.g., using higher concentrations of lower molecular weight polymers - may require consideration for these applications. Additional work is needed to determine $k_{w}$ values after gel placement in low-permeability rock.

\section{Permeability to Oil after Gel Placement}

Of course, the key to utilizing disproportionate permeability reduction is to identify conditions where a polymer or gel will reduce permeability to water much more than that to hydrocarbon. The previous section was concerned with whether water residual resistance factors were sufficiently high. This section will examine whether oil residual resistance factors can be sufficiently low. We also investigate how fast oil zones regain oil productivity after gel placement.

Concepts from Previous Work. Previous work ${ }^{5,25,26}$ revealed that gels can dehydrate during oil injection, thus causing disproportionate permeability reduction. Although oil cannot enter or flow through the gel matrix, pressure applied by the oil forces water to flow through and out from the gel. In locations where the gel has been compressed and dehydrated, oil forms "fingers" or "wormhole" pathways. These oil wormholes grow with time (and the entire gel structure continues to dehydrate with time under pressure), gradually increasing permeability to oil (note the solid circles in Fig. 3).

This time- and throughput-dependent behavior during oil flow through gel-filled cores has important consequences for the time required for wells to "clean up" or regain oil productivity after a gel treatment. ${ }^{22}$ For applications in production wells where hydrocarbon zones are not protected during placement, polymers and gelants necessarily penetrate some distance into the hydrocarbon zones. After the well is returned to production, oil can force its way through the gel to reach the well, but this process takes time. Our previous analysis suggested that the clean up time varies (1) with the cube of the distance of polymer or gelant penetration from the well, (2) inversely with pressure drawdown, and (3) inversely with $k_{w}$ in the gel-treated region. ${ }^{22}$

This last finding (i.e., that clean up time varies inversely with $k_{w}$ in the gel-treated region) inspired our current approach to optimizing disproportionate permeability reduction. A high $k_{w}$ value is desired to allow rapid dehydration and clean up of gel-treated areas during hydrocarbon flow. In contrast, a low $k_{w}$ value is desired to restrict flow from the water zones. Thus, an optimum $k_{w}$ value may be needed to maximize disproportionate permeability reduction.

For many of the experiments described in Table 1, oil (hexadecane for Entries 1-6 and Soltrol $130^{\mathrm{TM}}$ for Entries 7-17) was injected after gel placement and determination of $k_{w}$. The apparent permeability to oil $\left(k_{o}\right)$ for these experiments are summarized in Table 2 and are detailed in Figs. 6 through 13.

Effect of Pressure Gradient. The effects of pressure gradient on the $k_{o}$-versus- $P V$ curves are shown for gel in polyethylene cores in Fig. 6, in Berea sandstone cores in Fig. 7, and in fused silica cores in Fig. 8. For a given porous medium, the curves generally had similar shapes. However, in polyethylene and Berea, the curves shifted to greater $P V$ throughput values as the pressure gradients were increased. If pressure gradient had no effect on the gel structure, the curves should have overlapped. If the gel structure was compromised by applications of higher 
pressure gradients, the curves should have shifted to the left (i.e., higher permeabilities achieved with smaller throughput values.). Instead, the curves in Figs. 6 and 7 shifted right. It is possible that the differences are inherent variations associated with the gel and porous media. In fused silica (Fig. 8), the curves shifted left with increased pressure gradient.

Table 2-Ultimate $k_{o}$ and $F_{\text {rro }}$ during oil flow after gel placement.

\begin{tabular}{|c|c|c|c|c|c|c|c|c|}
\hline Entry & $\begin{array}{c}\text { Core } \\
\text { material }\end{array}$ & $\begin{array}{c}\text { Initial } \\
k, \\
\text { md }\end{array}$ & $\begin{array}{c}k_{o} \text { at } S_{w r} \\
\text { before gel, } \\
\text { md }\end{array}$ & $\begin{array}{c}\text { HPAM } \\
\text { in } \\
\text { gel, \% }\end{array}$ & $\begin{array}{c}d p / d l, \\
\text { psi/ft }\end{array}$ & $\begin{array}{c}\text { Final } \\
k_{o}, \\
\text { md }\end{array}$ & $\begin{array}{c}\text { Final } \\
F_{\text {rro }}\end{array}$ & $\begin{array}{c}\text { Final } \\
F_{\text {rrw }} \\
/ F_{\text {rro }}\end{array}$ \\
\hline 1 & Berea & 746 & 508 & 0.5 & 40 & 105 & 4.8 & 147 \\
\hline 2 & fused silica & 1,820 & 1,240 & 0.5 & 30 & 307 & 4.0 & 485 \\
\hline 3 & fused silica & 2,390 & 1,632 & 0.5 & 10 & 208 & 7.8 & 683 \\
\hline 4 & polyethylene & 6,400 & 6,400 & 0.5 & 100 & 515 & 12.4 & 1,210 \\
\hline 5 & polyethylene & 9,530 & 9,530 & 0.5 & 30 & 531 & 17.9 & 1,363 \\
\hline 6 & polyethylene & 15,270 & 11,410 & 0.5 & 10 & 637 & 17.9 & 983 \\
\hline 7 & Berea & 356 & $242^{*}$ & 0.5 & 2.7 & 209 & 1.2 & 19,800 \\
\hline 8 & Berea & 389 & 389 & 0.5 & 4.9 & 330 & 1.2 & 64,800 \\
\hline 9 & Berea & 100 & $68^{*}$ & 0.5 & 12 & 16.8 & 4.0 & 2,500 \\
\hline 10 & Berea & 40 & $27.2^{*}$ & 0.4 & 12 & 13.4 & 2.0 & 1,050 \\
\hline 11 & Berea & 274 & $186^{*}$ & 0.3 & 12 & 110 & 1.7 & 2,930 \\
\hline 12 & Berea & 98 & 132 & 0.5 & 21.6 & 60.1 & 2.2 & 2,010 \\
\hline 15 & sand pack & 8,100 & $8,100^{* *}$ & 0.5 & 1.5 & 1,840 & 4.4 & 63,400 \\
\hline 16 & polyethylene & 2,000 & $2,000^{* *}$ & 0.5 & 9.8 & 1,450 & 1.4 & 10,200 \\
\hline 17 & polyethylene & 3,200 & $3,200^{* *}$ & $0.125 \dagger$ & 9.2 & 1,052 & 3.0 & 4,630 \\
\hline
\end{tabular}

* Estimate based on Entry 1. ** Estimate based on Entries 4 and 5. $†$ xanthan instead of HPAM.

Interestingly, $k$ jumped from $\sim 0.2$ md to $\sim 2$ md between 0 and $0.1 P V$ in the polyethylene and silica cores. In Berea, $k_{o}$ at $0.1 P V$ was more similar to $k_{w}$ before oil flow. As a possible explanation, a rapid increase in $k_{o}$ may have been facilitated by imbibition of oil along the oilwet surfaces of the porous polyethylene. Upon first consideration, this explanation is not very satisfying for the same behavior in fused silica (Fig. 8), which we assumed was water-wet. However, since we did not measure the wetting characteristics of the fused silica cores, they may have been more oil-wet than we expected.

Effect of HPAM Content in the Gel. In Table 1, post-gel $k_{w}$ values increased with decreased polymer content in the gel-from $0.01 \mathrm{md}$ for the gel with $0.5 \%$ HPAM (Entry 9) to $0.055 \mathrm{md}$ for the gel with $0.3 \%$ HPAM (Entry 11). Fig. 9 reveals that for early oil throughput values, the $k_{o}$ values were qualitatively consistent with the post-gel $k_{w}$ trends. However, at higher throughput values, the separation of the curves became less evident. Fortunately, because the clean up behavior is dominated by the post-gel $k_{w}$ values and the early $k_{o}$ values, ${ }^{22}$ an advantage in clean up time can be realized by using gels with low polymer concentrations. However, this suggestion must be moderated by a realization that if the polymer content is too low, a pore-filling gel will not form. Instead, a weak gel or suspension of gel particles will form. 


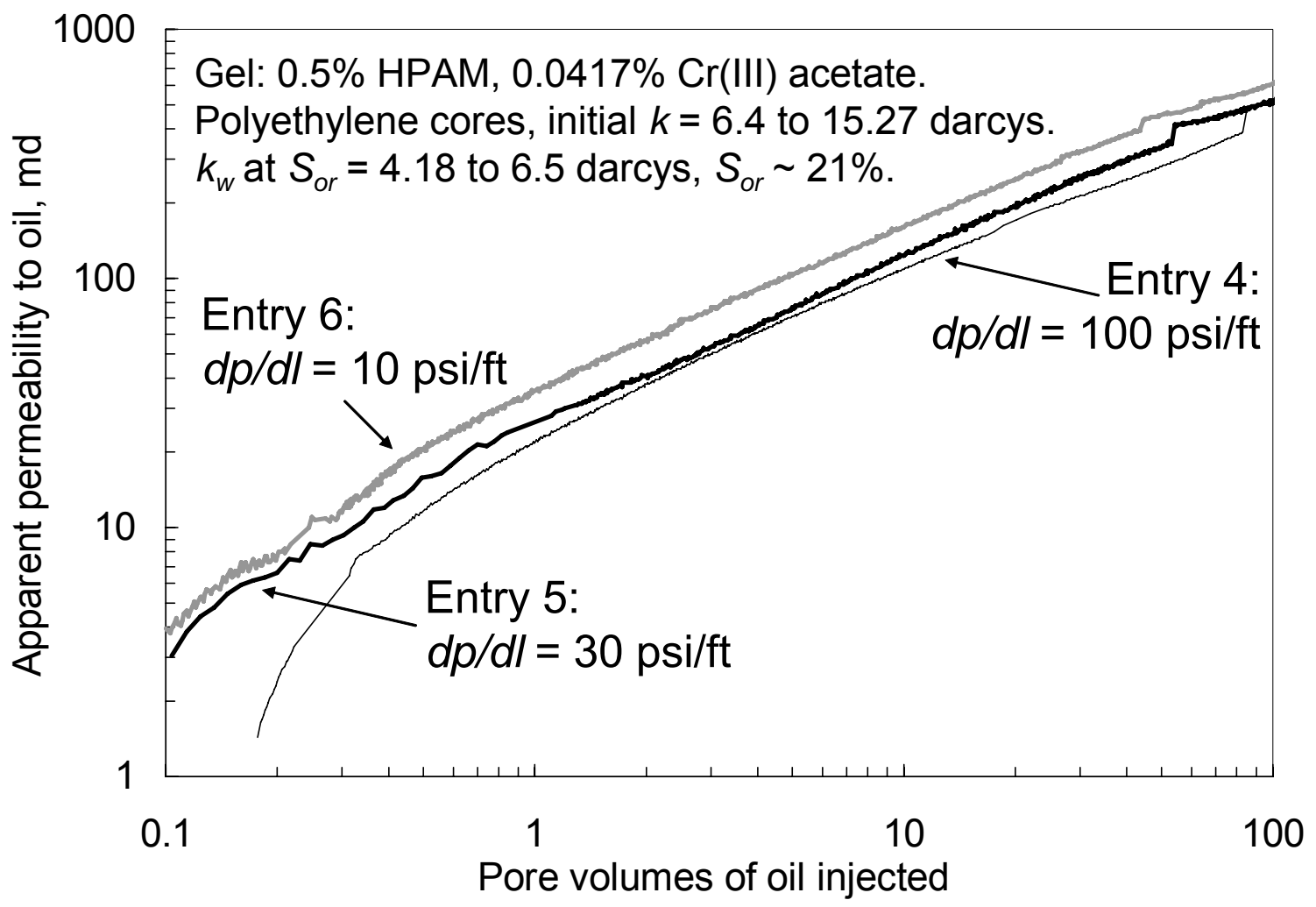

Fig. $6-k_{o}$ versus $P V$ and pressure gradient in polyethylene.

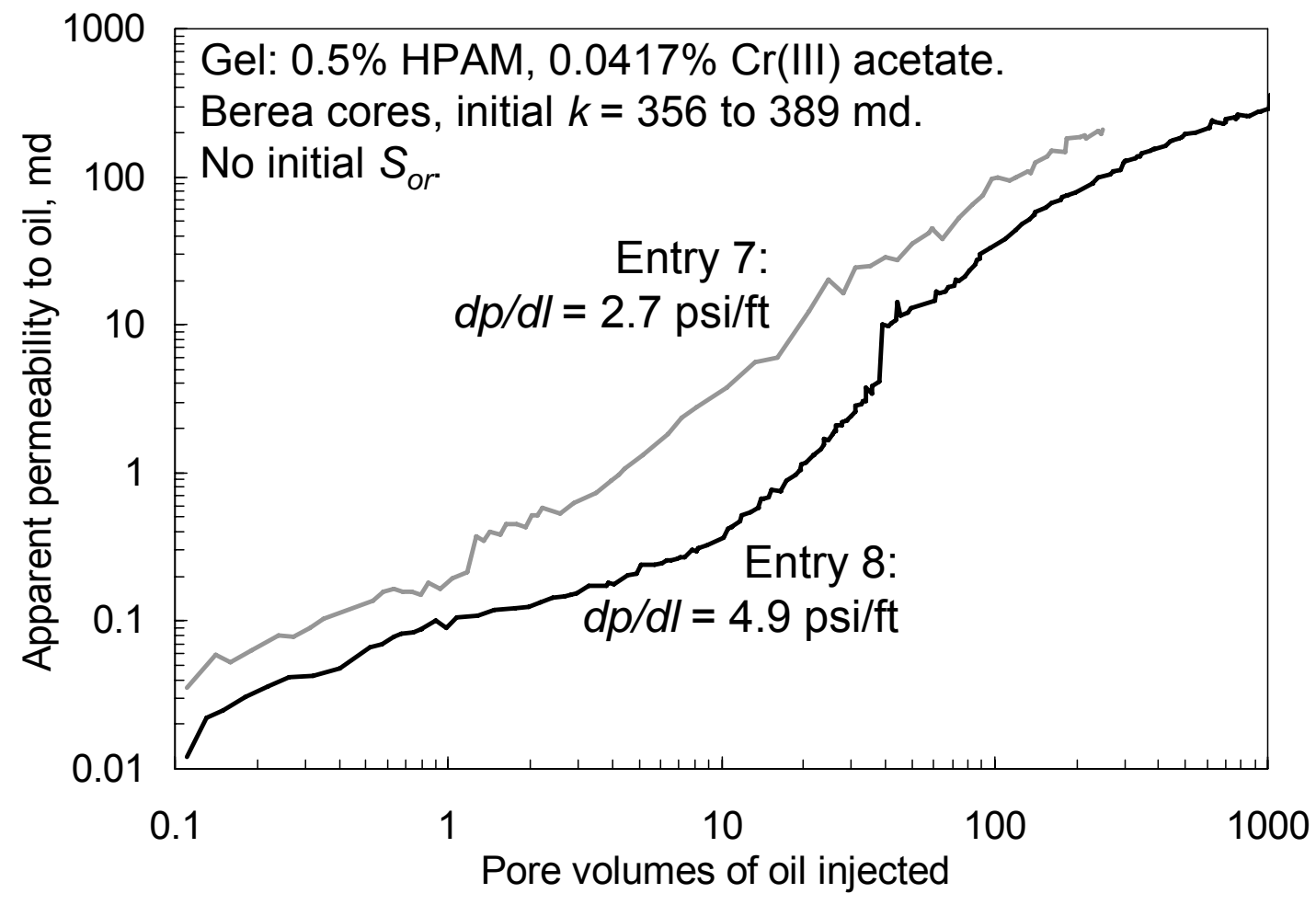

Fig. $7 \multimap k_{o}$ versus $P V$ and pressure gradient in Berea sandstone. 


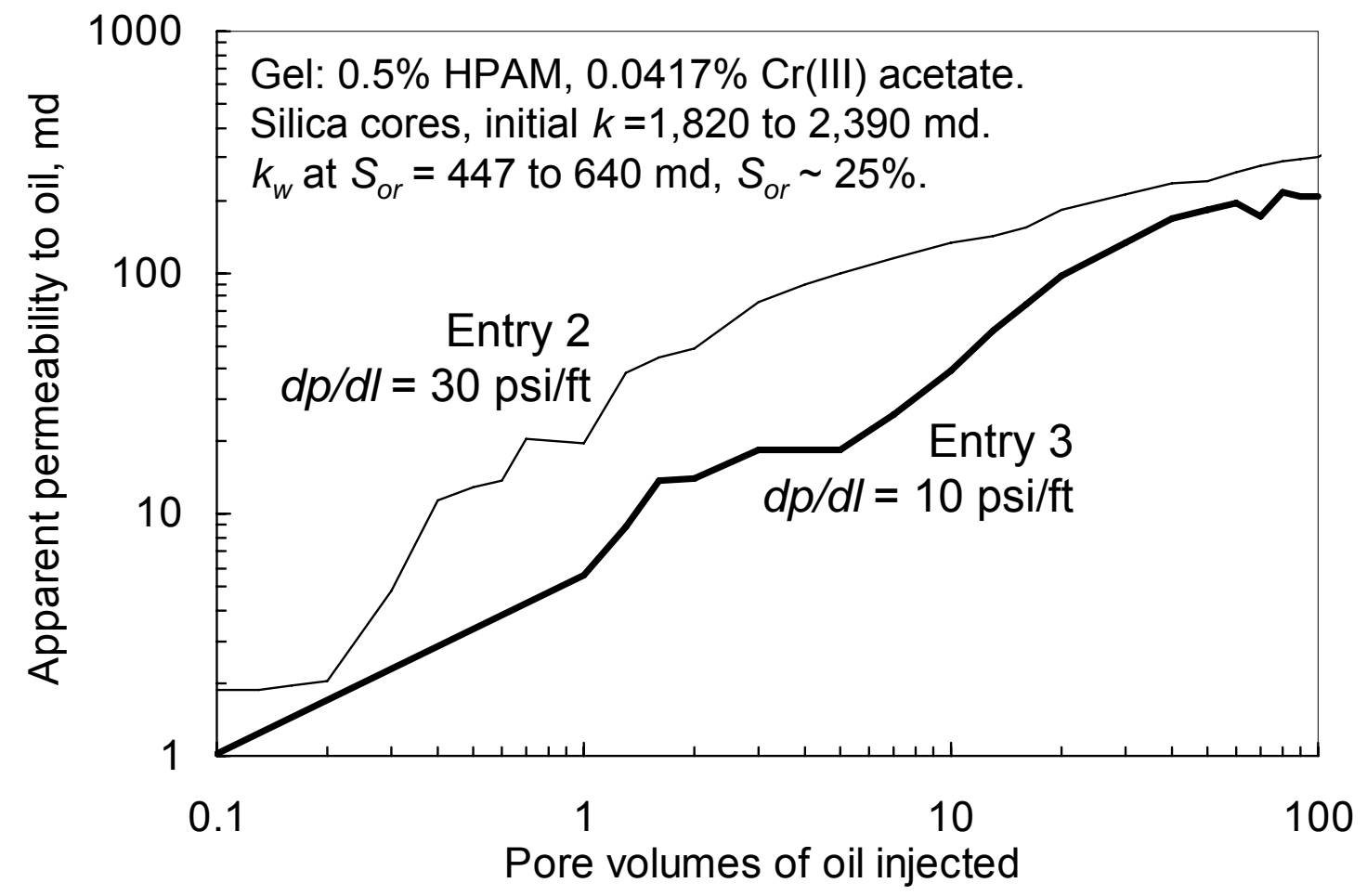

Fig. $8-k_{o}$ versus $P V$ and pressure gradient in fused silica.

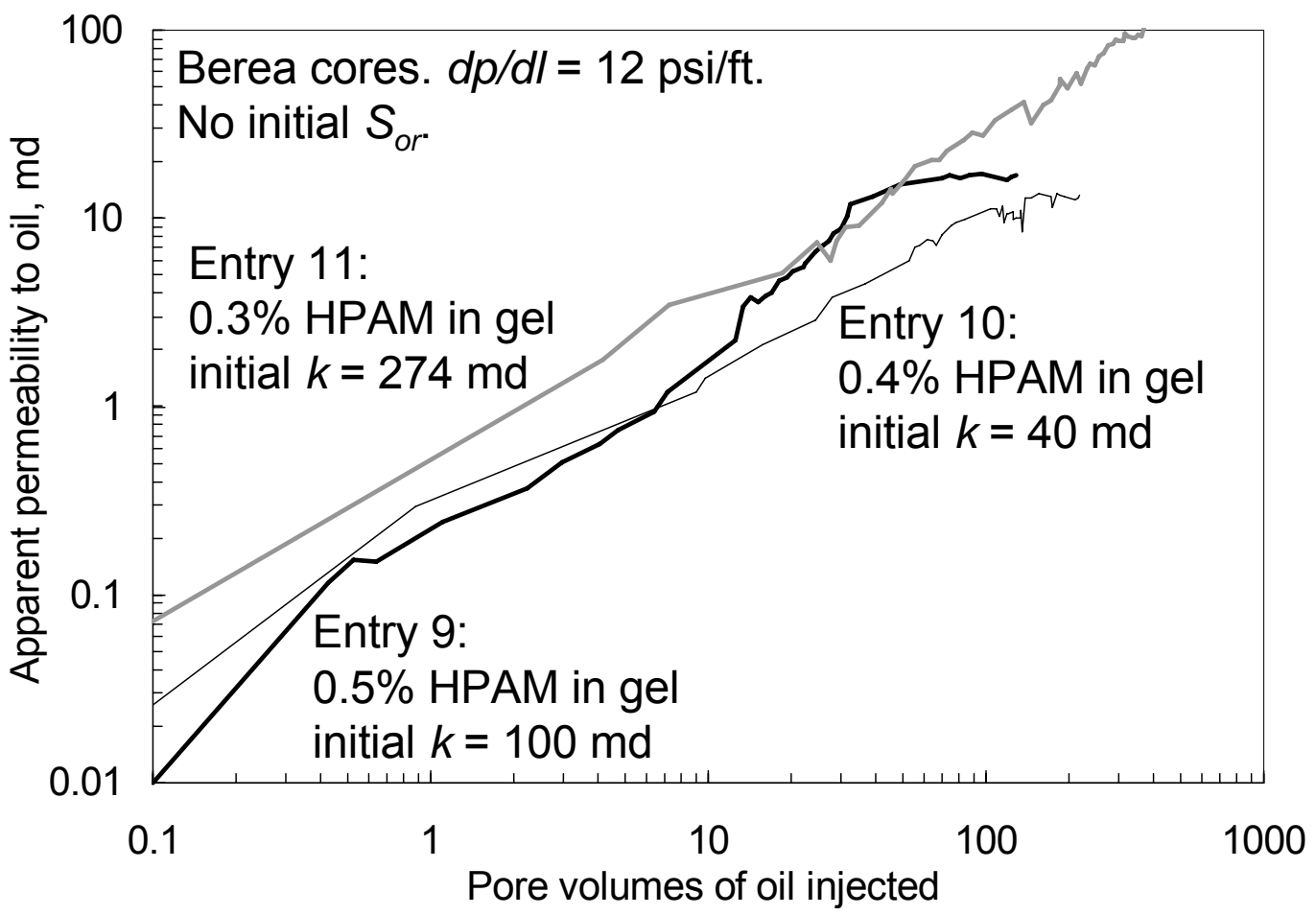

Fig. $9-k_{o}$ versus $P V$ for different HPAM concentrations. 
Effect of Initial $\boldsymbol{S}_{\text {or }}$. Figs. 10 and 11 show $k_{o}$ versus $P V$ for cases with and without an initial residual oil saturation. In Fig. 10, Berea sandstone cores were used with an initial (absolute) permeability (to water) of about $100 \mathrm{md}$. Both Berea cores had the same dimensions, and both were flooded with the same gel $\left(0.5 \%\right.$ HPAM, $\left.0.0417 \%, 1 \% \mathrm{NaCl}, 0.1 \% \mathrm{CaCl}_{2}\right)$. One core had no residual oil saturation before gelant placement (Entry 9). The other core (Entry 12) had a $S_{o r}$ of $31 \%$ before gelant placement and a $k_{w}$ value (at $S_{o r}$ ) of $31 \mathrm{md}$. Table 1 reveals that the $k_{w}$ values during brine injection (at $d p / d l=58 \mathrm{psi} / \mathrm{ft}$ ) immediately after gel placement were similar for the two cases $(0.01$ versus $0.007 \mathrm{md})$. Fig. 10 shows that the development of $k_{o}$ versus $P V$ was also similar for the two cases.

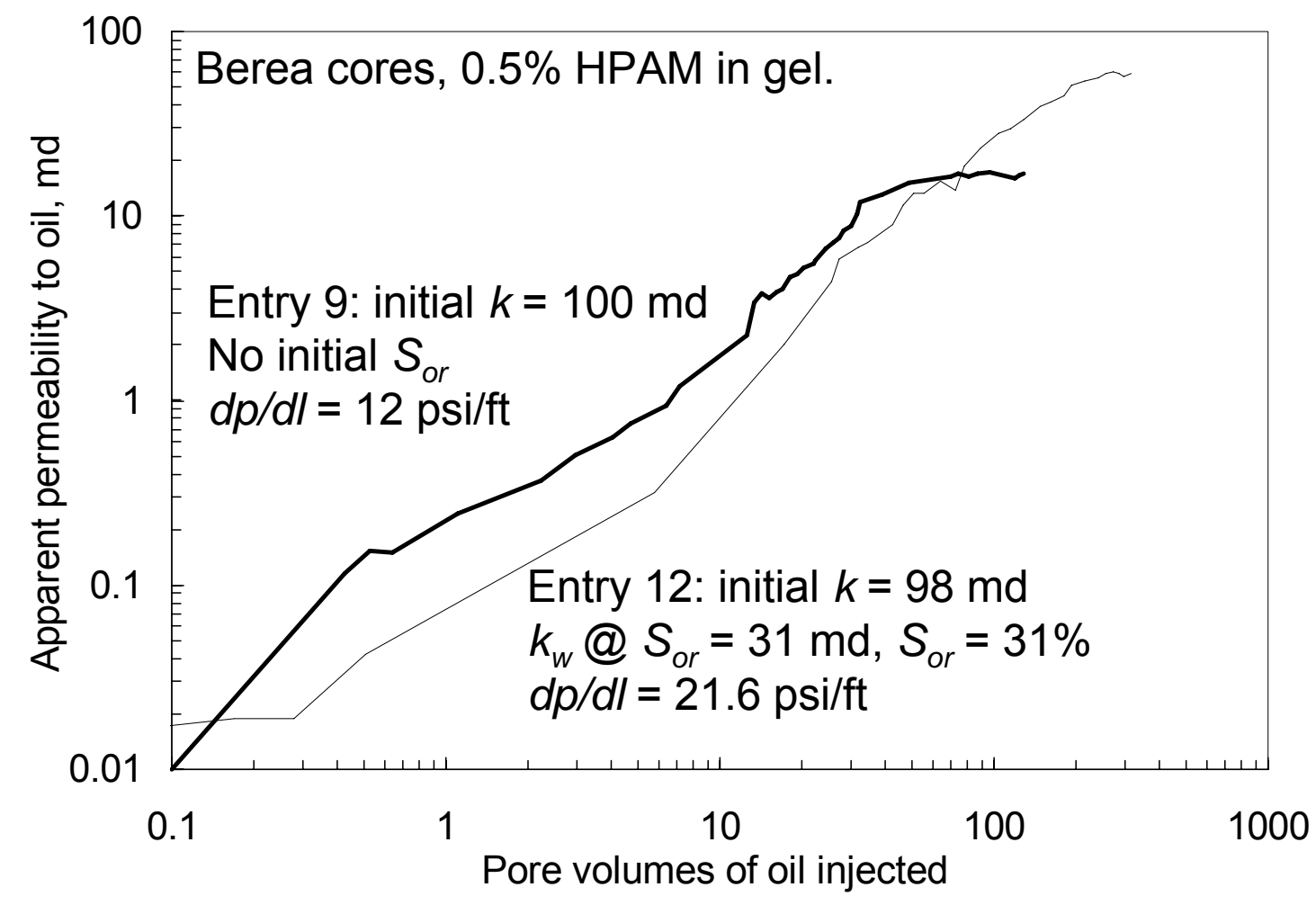

Fig. $10 — k_{o}$ with/without an intial $S_{o r}$ in Berea.

In contrast to the Berea cases, the presence of initial oil saturation had a significant effect in porous polyethylene. When $S_{o r}$ was present before gel placement (Entry 6 in Fig. 11), $k$ jumped from $0.37 \mathrm{md}$ to $4 \mathrm{md}$ between 0 and $0.1 P V$. Thus, in oil-wet porous polyethylene, the residual oil promoted a rapid initial increase in $k_{o}$. A similar jump did not happen for Entry 16 (where no residual oil was present before gelant placement) until about $10 P V$ of oil injection, when the $k_{o}$ curve increased to match the curve for Entry 6 . The large initial jump in $k_{o}$ was noted for the three polyethylene cores with $S_{o r}$ before gel (Fig. 6 and Entries 4, 5, and 6 in Table 1). It was also noted for the two fused silica cores (Fig. 8 and Entries 2 and 3 in Table 1), which we assumed (perhaps incorrectly) were strongly water-wet. In water-wet Berea, this rapid initial increase in $k_{o}$ was not observed, with or without the presence of residual oil before gelant placement (Fig. 10). 


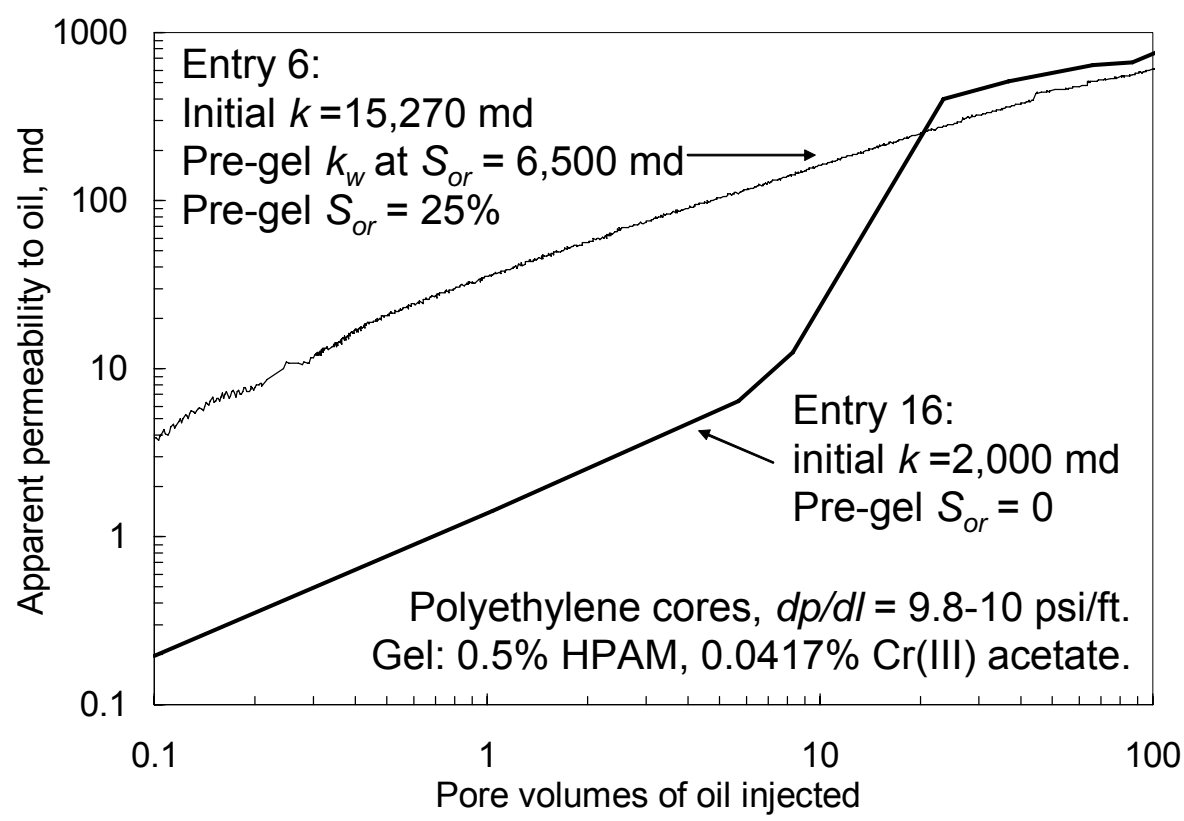

Fig. $11 — k_{o}$ with/without an initial $S_{o r}$ in polyethylene.

Effect of Porous Medium. Fig. 12 shows $k_{o}$ versus pore volume for different porous media (with no residual oil before gel placement). The three porous media, Berea sandstone, quartz sandpack, and porous polyethylene, show significantly different curves for $k_{o}$ versus pore volume. However, for Entries 8, 9, and 15 in Table 2 and Fig. 12 (i.e., the cases with strongly water-wet porous media) the early-throughput $k_{o}$ values were fairly similar.

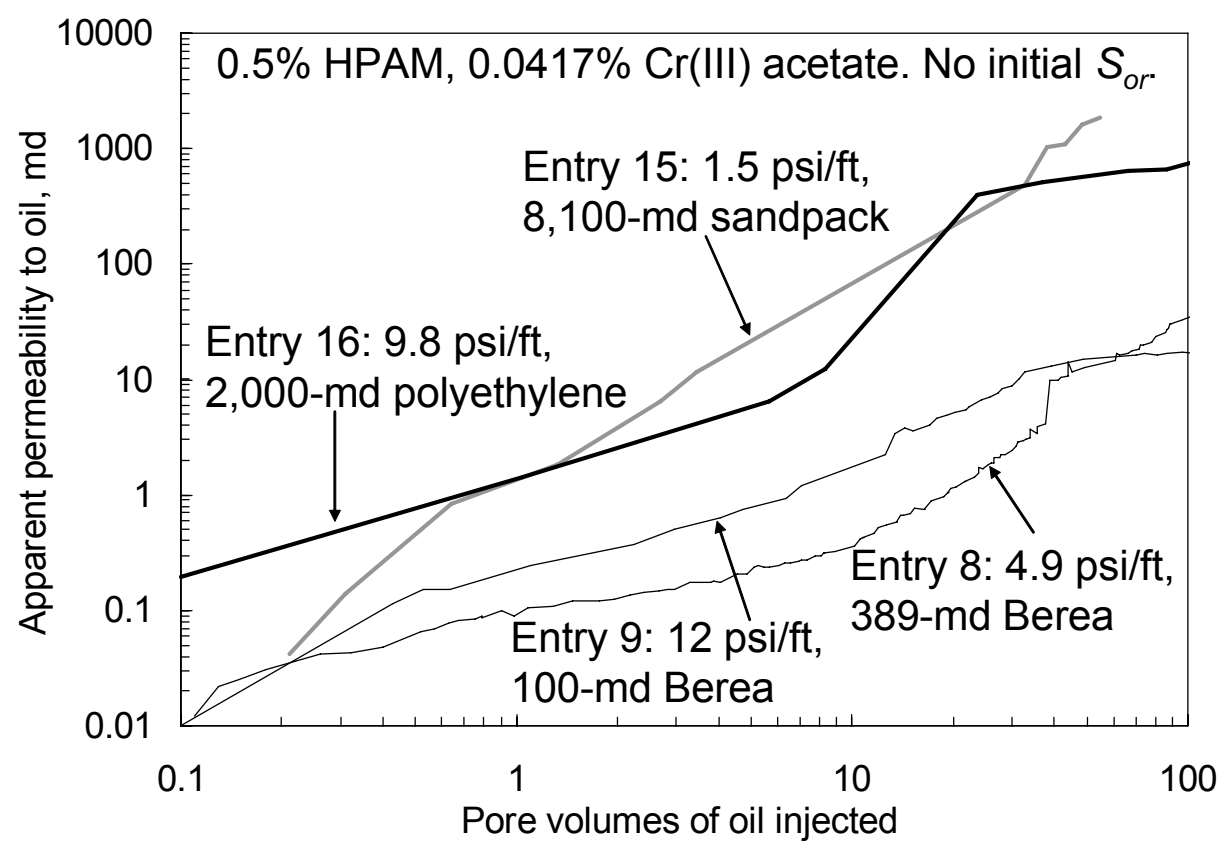

Fig. $12-k_{o}$ versus $P V$ for different porous media. 
Effect of Polymer Type in the Gel. For Entry 17, the gel contained $0.125 \%$ xanthan. During oil injection for Entry 17, $k_{o}$ increased steadily with throughput, as shown in Fig. 13. For comparison, results from a similar experiment (Entry 16) using a $\mathrm{Cr}(\mathrm{III})$-acetate-HPAM gel (with $0.5 \% \mathrm{HPAM}$ ) are also shown. The low-concentration, pore-filling $\mathrm{Cr}$ (III)-acetate-xanthan gel showed behavior similar to the $\mathrm{Cr}$ (III)-acetate-HPAM gel during the first $10 \mathrm{PV}$ of oil flow. Beyond $10 P V$, the curves deviated markedly, as the curve for Entry 16 experienced a sharp jump. Perhaps the xanthan gel adhered more tenaciously to the polyethylene pore walls than did the HPAM gel.

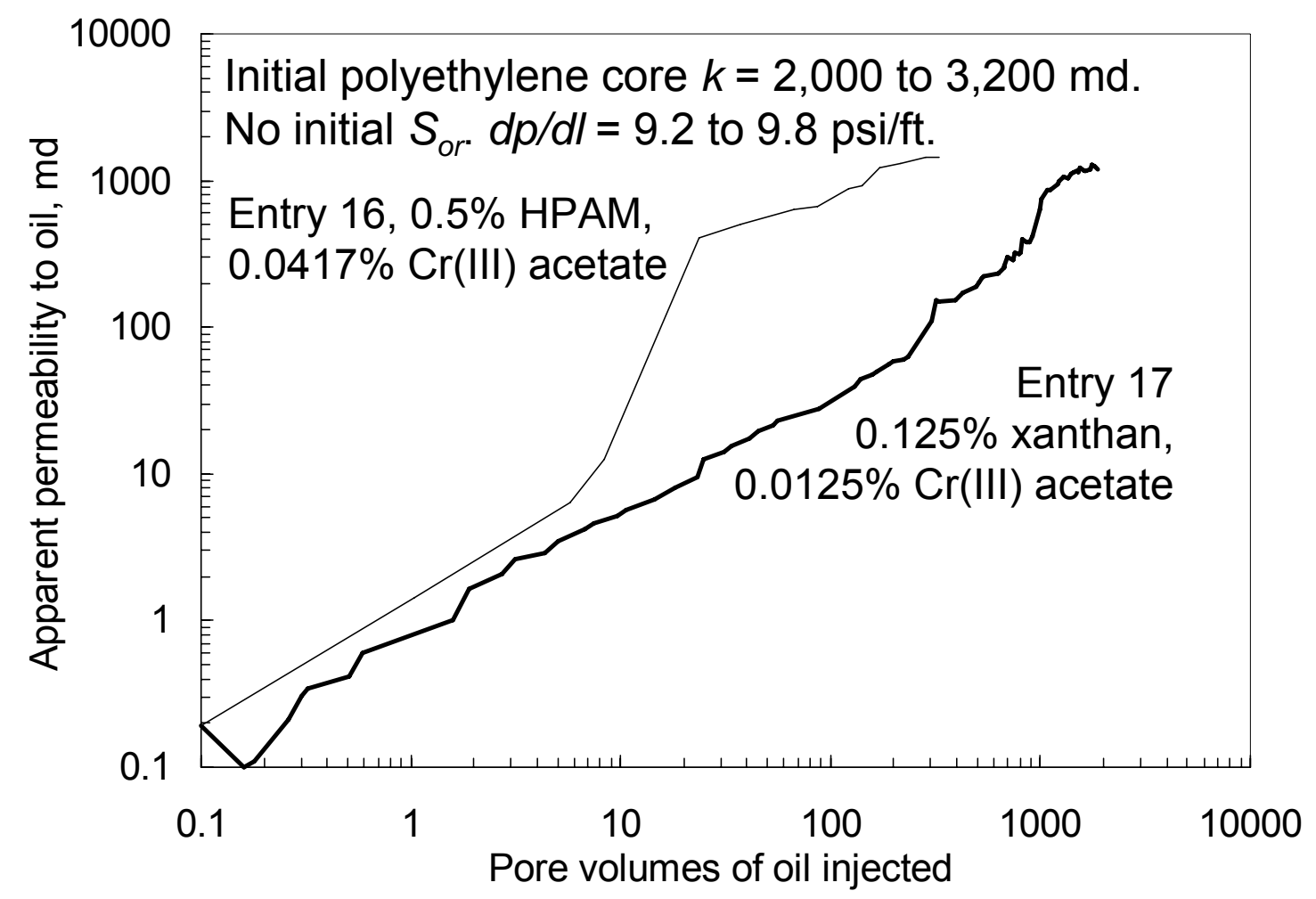

Fig. 13-Xanthan versus HPAM gels.

Are $\boldsymbol{F}_{\text {rro }}$ Values Low Enough? With radial flow, oil residual resistance factors must be less than 2 to mitigate damage caused by polymer or gel to oil productive zones. Can $F_{\text {rro }}$ values this low be attained using pore-filling gels? The seventh and eigth columns in Table 2 list the final $k_{o}$ and $F_{\text {rro }}$ values for each case. Typically, these values were measured after over $100 \mathrm{PV}$ of oil were injected. The lowest $F_{\text {rro }}$ value was 1.2. We found five cases where gels provided water residual resistance factors greater than 2,100 and ultimate $F_{\text {rro }}$ values of 2 or less (see Entries 7, 8, 10, 11, and 16 in Tables 1 and 2). These cases used gels with $0.3 \%$ to $0.5 \%$ HPAM. As noted above, $F_{\text {rro }}$ must be less than 2 for radial flow treatments where hydrocarbon zones are not protected during gel placement. So, our recent results provide hope that our current approach will identify a gel that can successfully and reliably treat either fractured or unfractured production wells without zone isolation.

For linear flow applications (wells that intersect fractures), our main requirement was that the gel reduce permeability to water much more than that to oil. The last column in Table 2 lists the 
ratio, $F_{r r w} / F_{r r o}$. These ratios range from 147 to 64,800 . These values would be very acceptable for applications in very permeable media. Unfortunately, fractures and fracture-like features are less likely to present channeling problems as the permeability of the media increases. The greatest need for high $F_{r r w} / F_{r r o}$ ratios exists in tight rock. As mentioned earlier, if the $F_{r r w}$ value is too low when treating a fracture, the gelant must leakoff a substantial distance from the fracture faces. In addition to the expense of requiring large gelant volumes, this situation may be limiting because many polymer-based gelants cannot penetrate into tight rock. ${ }^{18}$ On a positive note, the case for Entry 10 (using gel with $0.4 \%$ HPAM in 40-md Berea) exhibited $F_{r r w}=2,110$, $F_{r r o}=2.0$, and $F_{r r w} / F_{r r o}=1,050$. These values would be quite acceptable for applications in either fractured or unfractured wells. Since they were measured in 40-md rock, we see hope that our approach will identify a gel that can successfully and reliably treat either fractured or unfractured production wells without zone isolation.

\section{How Fast Will Oil Zones Clean Up?}

Previous Approach. In Ref. 22, a means was described to estimate how rapidly productivity can be restored in an oil zone that was invaded by a polymer or gel. A simple mobility-ratio model was used to predict clean up times for both fractured and unfractured wells after a gel treatment. Conventional relative permeability equations were used (Eqs. 3 and 4).

$$
\begin{aligned}
& k_{r w}=k_{r w}{ }^{o}\left(\left(S_{w}-S_{w r}\right) /\left(1-S_{o r}-S_{w r}\right)\right)^{n w} . \\
& k_{r o}=k_{r o}{ }^{o}\left(\left(1-S_{o r}-S_{w}\right) /\left(1-S_{o r}-S_{w r}\right)\right)^{n o}
\end{aligned}
$$

The time to restore productivity to a gel-treated oil zone (1) was similar for radial versus linear flow, (2) varied roughly with the cube of distance of gel penetration, (3) varied inversely with pressure drawdown, (4) varied inversely with the $k_{w}$ at $S_{o r}$ in the gel-treated region, and (5) was not sensitive to the final $k_{o}$ at $S_{w r}$. Although $k_{o}$ at $S_{w r}$ (after gel placement) had no effect on the clean up time, it strongly affected how much of the original oil productivity was ultimately regained. The results from this model were described quite well using an empirical equation (Eq. 5), so long as the distance of gelant penetration was not too small. Eq. 5 predicts the level of oil productivity that may be regained $\left(q_{\text {oil }} / q_{\text {end }}\right)$ as a function of time $(t$ in days), distance of gelant penetration into the porous rock ( $L_{p}$ in $\mathrm{ft}$ ), permeability to water in the gel-treated region ( $k_{w}$ in $\mathrm{md})$, pressure drawdown ( $\Delta p$ in psi), and fluid viscosities $\left(\mu_{o}\right.$ and $\left.\mu_{w}\right)$.

$$
q_{\text {oil }} / q_{\text {end }}=(2 / \pi) \arctan \left[\left(64 t \Delta p k_{w} \mu_{w} /\left(\mu_{o} L_{p}^{3}\right)\right)^{\pi / 4}\right]
$$

New Approach. The approach taken in Ref. 22 and Eq. 5 assumed that recovery of oil productivity was dominated by the inherent permeability of the gel to water. It neglected the detailed shapes of the $k_{o}$-versus- $P V$ curves in Figs. 6-13. Consequently, an effort was made to consider how clean up time was influenced by these curves. A power regression worked reasonably well in fitting the curves in Figs. 6-13. Columns 8-10 in Table 3 list regression parameters associated with fitting the data with the equation,

$$
k_{o}=k_{w}+B(P V)^{n}
$$


Table 3-Actual versus predicted times to recover $50 \%$ of ultimate $k_{o}$.

\begin{tabular}{|c|c|c|c|c|c|c|c|c|c|c|c|c|}
\hline \multicolumn{6}{|c|}{ Core properties } & \multirow[b]{2}{*}{$\begin{array}{c}\text { Post- gel } \\
k_{w}, \text { md }\end{array}$} & \multicolumn{3}{|c|}{$\begin{array}{l}\text { Parameters from power } \\
\text { regression, (Eq. 6) }\end{array}$} & \multicolumn{3}{|c|}{$\begin{array}{c}\text { Time to reach } 50 \% \text { of } \\
\text { ultimate } k_{o} \text {, days }\end{array}$} \\
\hline Entry & $\begin{array}{c}\text { Core } \\
\text { material }\end{array}$ & $\begin{array}{l}\text { Initial } k \text {, } \\
\text { md }\end{array}$ & $\begin{array}{c}S_{o r} \\
\text { present? }\end{array}$ & $\begin{array}{l}d p / d l, \\
\mathrm{psi} / \mathrm{ft}\end{array}$ & $\begin{array}{c}\text { Core } \\
\text { length, } \\
\mathrm{ft}\end{array}$ & & $B$ & $n$ & $R^{2}$ & Actual & $\begin{array}{c}\text { Old } \\
\text { model }\end{array}$ & $\begin{array}{l}\text { New } \\
\text { model }\end{array}$ \\
\hline 1 & Berea & 746 & yes & 40 & 0.256 & 0.17 & 13 & 0.52 & 0.956 & 0.37 & 0.14 & 0.40 \\
\hline 2 & fused silica & 1,820 & yes & 30 & 0.231 & 0.23 & 17.2 & 0.73 & 0.923 & 0.08 & 0.13 & 0.6 \\
\hline 3 & fused silica & 2,390 & yes & 10 & 0.244 & 0.12 & 8.9 & 0.72 & 0.972 & 0.9 & 0.8 & 3.5 \\
\hline 4 & polyethylene & 6,400 & yes & 100 & 0.238 & 0.32 & 19.8 & 0.72 & 0.971 & 0.84 & 0.03 & 0.24 \\
\hline 5 & polyethylene & 9,530 & yes & 30 & 0.245 & 0.24 & 25 & 0.68 & 0.997 & 1.2 & 0.14 & 0.68 \\
\hline 6 & polyethylene & 15,270 & yes & 10 & 0.256 & 0.37 & 31 & 0.69 & 0.991 & 2.1 & 0.28 & 1.6 \\
\hline 7 & Berea & 356 & $\overline{\text { no }}$ & 2.7 & 0.438 & 0.015 & 0.244 & 0.86 & 0.941 & 162 & $\overline{45}$ & 330 \\
\hline 8 & Berea & 389 & no & 4.9 & 0.462 & 0.005 & 0.05 & 1.26 & 0.955 & 255 & 81 & 363 \\
\hline 9 & Berea & 100 & no & 12 & 0.104 & 0.01 & 0.183 & 1.1 & 0.978 & 7.2 & 3.9 & 10.2 \\
\hline 10 & Berea & 40 & no & 12 & 0.104 & 0.019 & 0.22 & 0.86 & 0.985 & 8.4 & 2.2 & 10.2 \\
\hline 11 & Berea & 274 & no & 12 & 0.104 & 0.055 & 0.45 & 0.91 & 0.991 & 4.4 & 0.73 & 5.9 \\
\hline 12 & Berea & 98 & yes & 21.6 & 0.104 & 0.007 & 0.10 & 1.17 & 0.988 & 9.5 & 1.8 & 10.8 \\
\hline 15 & sand pack & 8,100 & no & 1.5 & 0.405 & 0.029 & 1.13 & 1.84 & 0.996 & 94 & $\overline{42}$ & 129 \\
\hline 16 & polyethylene & 2,000 & no & 9.8 & 0.229 & 0.14 & 2.2 & 1.2 & 0.947 & 2.5 & 0.4 & 4.5 \\
\hline 17 & polyethylene & 3,200 & no & 9.2 & 0.245 & 0.23 & 0.743 & 0.88 & 0.992 & 25 & 0.54 & 20.6 \\
\hline
\end{tabular}


This equation was used as the basis for our new model. The last column in Table 3 lists the calculated time (i.e., the estimated clean up time) for $k_{o}$ to reach $50 \%$ of its final value. For comparison, the second to last column in Table 3 lists clean up times using our previous model. ${ }^{22}$ Calculated clean up times from the new method were consistently greater than those from the old method and were generally closer to the actual laboratory values (third to last column in Table 3 ), except for the cases using fused silica cores.

Using parameters associated with Entry 1 in Tables 1-3, Fig. 14 was generated to compare predictions from the two models as a function of distance of gelant penetration into porous rock. For the old model, the saturation exponents, $n w$ and no were $2, S_{w r}$ was $0.3, S_{o r}$ was $0.3, k_{w}$ (at $S_{o r}$ after gel placement) was $0.17 \mathrm{md}, k_{o}$ (ultimate value after gel placement and $100 \mathrm{PV}$ of oil injection) was $105 \mathrm{md}$, oil viscosity, $\mu_{o}$, was $3.34 \mathrm{cp}$, and water viscosity was $1 \mathrm{cp}$. For the new model, $k_{w}$ was $0.17 \mathrm{md}, B$ was 13 and $n$ was 0.52 .

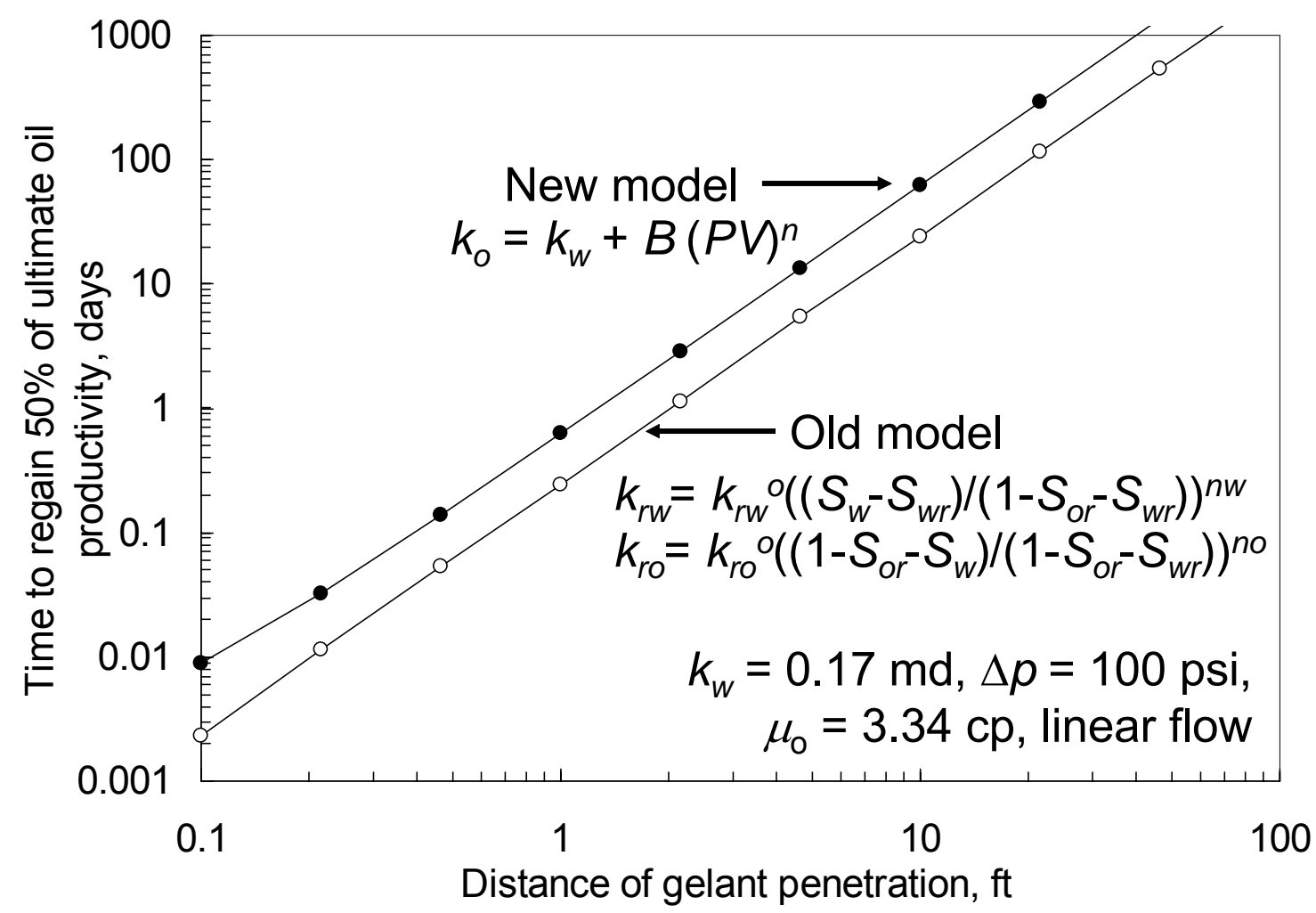

Fig. 14 Predicted clean up times: old versus new models.

For a given distance of gelant penetration, predicted clean up times from the new model were consistently about 2.5 times longer than those from the old model. For the conditions in Fig. 14, the new model indicates that the distance of gelant penetration must be less than $4 \mathrm{ft}$ in order to keep the clean up time less than 10 days. For both models, the clean up time increased with the square of the distance of gelant penetration. In contrast in Ref. 22, we reported that the clean up time should increase with the cube of the distance of gelant penetration. A detailed reconsideration of the old analysis revealed that the apparent dependence of clean up time on length was influenced by the saturation exponents used and the range of lengths examined. 
Laboratory results and the predictions from both models suggest that clean up time is strongly influenced by the magnitute of $k_{w}$ immediately after gel formation. Consequently, there are three main choices to speed clean up time after a gel treatment: (1) limit gelant penetration into porous rock to small distances (i.e., no more than a few feet with current gels), (2) maximize pressure drawdown for the well, and (3) attempt to increase $k_{w}$ in the gel-treated rock. The first two options should be employed as much as practical, but limits exist on how much they can be exploited. Our future work will focus on the third option: identifying a gel that provides higher, stable $k_{w}$ values in the gel-treated rock.

Reliability. Reliability and reproducibility of performance are central issues for field applications of polymers and gels. Fig. 4 and Column 9 of Table 1 indicate that pore-filling gels can consistently reduce $k_{w}$ to low values, regardless of the nature of the porous medium. Figs. 613 and Column 8 of Table 2 reveals that given enough oil throughput, pore-filling gels consistently provide low $F_{\text {rro }}$ values - although not necessarily values below two. The main area of variability is the time or throughput dependence of achieving low $F_{\text {rro }}$ values. If the distance of gelant penetration into porous rock is small, this variability will not matter, since all clean up times will be fast. However for intermediate distances of penetration (i.e., multiple feet), the variability may be of concern. For a given type of porous medium, the curves for $k_{o}$ versus $P V$ were fairly similar (see Figs. 6-10). Consequently, variability of clean up times may be manageable for wells within a given field. Since the greatest differences amoung the curves for $k_{o}$ versus $P V$ were seen when comparing different porous media (Fig. 12), significant variations in clean up times might be expected after gel treatments in wells from different fields.

\section{Second Water Flow after Oil Flow}

Water was injected again after the oil-flow experiments described in Table 2 and in Figs. 6-13. In all cases, the permeability to water stabilized quite quickly. Column 7 in Table 4 lists $k_{w}$ values for these experiments. The last column in Table 4 converts the $k_{w}$ values to water residual resistance factors. (For Entries 7-11 and 15-17, the initial core permeability to water was used when calculating $F_{r r w}$ values, because $k_{w}$ values were not measured at $S_{o r}$ before gel placement.) For Entries 1-5 in Table 4, the second $k_{w}$ values were quite low- 0.22 to $1.17 \mathrm{md}$. In most cases, these values were higher than the first $k_{w}$ values after gel placement (Table 1), but were still very low relative to the $k_{o}$ values (Table 2). An explanation was provided in Refs. 25 and 26. (The explanation involves trapping of high residual oil saturations.)

For Entries 6-17 in Table 4, the second $F_{r r w}$ values were between 126 and 29,400 times less than the values before oil injection (compare the last columns of Tables 1 and 4). Thus, extended oil injection caused substantial damage to the gel for these cases. Interestingly, this damage was much less severe for the cases associated with Entries 1-5, where residual oil was present during gel placement. For Entries 1-5, the second $F_{r r w}$ values were between one and seven times less than the values measured before oil injection.

The results in this section (i.e., for water flow following both gel placement and subsequent oil flow) are generally more of academic interest than of practical interest. After a polymer or gel treatment has been applied, brine is the first flowing fluid to contact the polymer or gel in the water zones, so the results from our "Permeability to Water after Gel Placement" section are of direct practical interest. Similarly, oil is the first flowing fluid to contact the polymer or gel in the 
oil zones, so the results from our "Permeability to Oil after Gel Placement" section are also of direct practical interest. The results from this current section could have practical application mainly if an oil zone becomes watered out after being treated by a polymer or gel.

Table 4-Ultimate $k_{w}$ and $F_{r r w}$ during the second water flow after gel placement.

\begin{tabular}{|c|c|c|c|c|c|c|c|}
\hline Entry & $\begin{array}{c}\text { Core } \\
\text { material }\end{array}$ & $\begin{array}{c}\text { Initial } k, \\
\mathrm{md}\end{array}$ & $\begin{array}{c}k_{w} \text { at } S_{o r} \\
\text { before gel, } \mathrm{md}\end{array}$ & $\begin{array}{c}\text { HPAM in } \\
\text { gel, } \%\end{array}$ & $\begin{array}{c}d p / d l, \\
\mathrm{psi} / \mathrm{ft}\end{array}$ & $\begin{array}{c}2 \mathrm{nd} k_{w}, \\
\mathrm{md}\end{array}$ & $\begin{array}{c}\text { 2nd } \\
F_{r r w}\end{array}$ \\
\hline 1 & Berea & 746 & 120 & 0.5 & 40 & 1.11 & 108 \\
\hline 2 & fused silica & 1,820 & 447 & 0.5 & 30 & 0.22 & 2,030 \\
\hline 3 & fused silica & 2,390 & 640 & 0.5 & 10 & 0.35 & 1,830 \\
\hline 4 & polyethylene & 6,400 & 4,810 & 0.5 & 100 & 0.74 & 6,500 \\
\hline 5 & polyethylene & 9,530 & 5,860 & 0.5 & 30 & 1.17 & 5,008 \\
\hline 6 & polyethylene & 15,270 & 6,500 & 0.5 & 10 & 106 & 61.3 \\
\hline 7 & Berea & 356 & $*$ & 0.5 & 13.7 & 42 & 8.5 \\
\hline 8 & Berea & 389 & $*$ & 0.5 & 30 & 26 & 15 \\
\hline 9 & Berea & 100 & $*$ & 0.5 & 58 & 2.5 & 40 \\
\hline 10 & Berea & 40 & $*$ & 0.4 & 58 & 2.4 & 16.7 \\
\hline 11 & Berea & 274 & $*$ & 0.3 & 58 & 18.5 & 14.8 \\
\hline 12 & Berea & 98 & 31 & 0.5 & 58 & 2.2 & 14.1 \\
\hline 15 & sand pack & 8,100 & $*$ & 0.5 & 14.8 & 850 & 9.5 \\
\hline 16 & polyethylene & 2,000 & $*$ & 0.5 & 24.5 & 860 & 2.3 \\
\hline 17 & polyethylene & 3,200 & $*$ & $0.125 \dagger$ & 24.5 & 210 & 15.2 \\
\hline
\end{tabular}

* Since no residual oil was present, $F_{r r w}$ was calculated using the initial core permeability. $†$ xanthan instead of HPAM.

\section{Conclusions}

We investigated the merits of pore-filling gels in providing disproportionate permeability reduction.

1. For porous media with pre-gel $k_{w}$ (at $S_{o r}$ ) ranging from 120 to $6,500 \mathrm{md}$, one strong porefilling $\mathrm{Cr}$ (III)-acetate-HPAM gel (with $0.5 \%$ HPAM) consistently reduced $k_{w}$ to about 0.24 md (ranging from 0.12 to $0.37 \mathrm{md}$ ). In contrast, in Berea sandstone with $k_{w}$ (at $S_{o r}$ ) ranging from 222 to $363 \mathrm{md}$, a weak gel (with $0.18 \%$ polymer) exhibited a much wider range of postpolymer $k_{w}$ values - from 0.75 to $202 \mathrm{md}$. Thus, strong pore-filling gels can provide greater reliability and behavior that is insensitive to the initial rock permeability.

2. With sufficient oil throughput, pore-filling gels can be dehydrated, thus increasing permeability to oil. We found several formulations where gels provided water residual resistance factors greater than 2,000 and ultimate $F_{\text {rro }}$ values of 2 or less. These results provide hope that our current approach will identify gels that can successfully and reliably treat either fractured or unfractured production wells without zone isolation.

3. Significant oil throughput was required to achieve low $F_{\text {rro }}$ values, suggesting that gelant penetration into porous rock must be small (a few feet or less) for existing pore-filling gels to provide effective disproportionate permeability reduction. 


\section{GUIDELINES FOR RELATIVE PERMEABILITY MODIFICATION TREATMENTS}

Relative permeability modification (RPM) is a property that is exploited during certain oilfield water-shutoff (WSO) treatments and a property whereby many water-soluble polymers and aqueous polymer gels reduce the permeability to water flow to a greater extent than to oil or gas flow. Refs. 2, 11, and 27-44 are illustrative literature references that discuss the RPM phenomenon. RPM WSO treatments are applicable to both oil and gas production wells.

RPM is also referred to as disproportionate permeability reduction (DPR). Some practitioners reserve the term "DPR" for relatively strong polymer gels that impart a large degree of disproportionate permeability reduction and a large reduction in water permeability. These practitioners reserve the term "RPM" for systems such as solutions of water-soluble polymers or relatively "weak" gels that impart more subtle disproportionate permeability reductions and more subtle reductions in water permeability. However, in this chapter, the terms RPM and DPR will be considered synonyms. At times in the literature, DPR and RPM have also been referred to as "selective permeability reduction" and "selective permeability blocking."

In this chapter, the term "WSO treatment" refers to a chemical treatment that is applied (to an oil or gas producing reservoir) to either reduce or totally shut off water production from a well.

Historically, RPM/DPR is a phenomenon that was believed limited to fluid flow in matrix rock porous media. More recently, it has been reported that certain relatively strong WSO gels impart RPM/DPR to fluid flow within gel-filled fractures. ${ }^{45}$ However, because such relatively strong gels also significantly reduce the permeability to oil flow in fractures, these gels are better characterized as total shutoff gels than as RPM/DPR gels.

DPR is only of value for water-shutoff treatments applied to production wells. DPR has little, or no, value for application from the injection-well side.

A distinction that has not been clearly made in the past is RPM/DPR treatments that promote long-term ("permanent") versus short-term (transient) WSO. In this chapter, "long-term" means months to years and hopefully for the economic life of the treated well, and "short-term" or "transient" means hours up to a month or two (often hours to days). Long-term and short-term RPM/DPR treatments will be discussed and differentiated in this chapter. This distinction helps to explain some of the historically disappointing field results of these treatments.

The objectives of this chapter are as follows. First, we will outline when, where, and how RPM/DPR treatments can be successfully applied. Second, issues, potential pitfalls, and limitations relating to the successful application of RPM/DPR treatments will be reviewed.

\section{Background}

Historical Review. The ability of acrylamide polymers to impart RPM/DPR to water and oil flow in porous media was recognized as early as 1964 by Sandiford ${ }^{27}$ and 1973 by White et al. ${ }^{28}$ The mechanism(s) by which numerous water-soluble polymers and aqueous gels impart RPM and DPR has been the subject of a number of investigations - with Refs. 5, 21, 26, 46-56 being representative. More recently, a plausible mechanism was proposed that explains how 
chromium(III)-carboxylate/acrylamide-polymer (CC/AP) gels impart DPR. ${ }^{25}$ A detailed discussion of the mechanism by which water-soluble polymers and aqueous polymer gels impart $\mathrm{RPM} / \mathrm{DPR}$ is beyond the scope of this chapter.

Historically, a large number of ineffective, underperforming, and/or disappointing RPM/DPR water-shutoff treatments were applied by the petroleum industry. ${ }^{38,39,11,57-63}$ This chapter will provide insight into the reasons for the historically uninspiring field success rate for RPM/DPR treatments.

Why RPM/DPR Treatments Are Attractive. The reason that there is so much active interest in the petroleum industry regarding bullheadable DPR water-shutoff treatments is that they normally do not require the use of mechanical zone isolation during treatment-fluid placement. In contrast, when applied to wells of matrix rock reservoirs involving radial flow, conventional (relatively strong and total-fluid-shutoff) polymer-gel WSO treatments normally require the use of mechanical zone isolation during treatment placement. ${ }^{70}$ Mechanical zone isolation often requires costly workover operations. In addition, the use of mechanical zone isolation during water-shutoff-treatment placement is normally not feasible when the well possesses a slottedliner or gravel-pack completion or when the well involves a sub-sea tieback flow line. Presently, $\mathrm{RPM} / \mathrm{DPR}$ treatments are a technology that is in vogue within the industry, and many individuals and organizations are attempting to develop and exploit these treatments.

RPM/DPR Does Occur. When numerous of the early RPM/DPR treatments did not perform as well as expected, a number of oil-industry professionals questioned whether RPM/DPR actually occurs. As it turns out, it does. ${ }^{1-63}$ Thus, the challenge is to learn when, where, and how RPM/DPR can be successfully employed in WSO treatments. Addressing this challenge will be the focus of the remainder of this chapter.

\section{Ideal RPM/DPR Treatments}

As used in this chapter, "ideal" RPM/DPR treatment means the following. First, an ideal $\mathrm{RPM} / \mathrm{DPR}$ treatment does not reduce oil permeability at all in the volume of matrix reservoir rock where it is placed. Second, in the field setting, an ideal RPM/DPR treatment does not promote any reduction in the post-treatment oil-production rate.

In this chapter when considering polymer-alone and weak-gel WSO treatments in unfractured reservoirs, we will, for the most part, discuss ideal RPM/DPR treatments. Unfortunately, an ideal RPM/DPR technology does not yet exist commercially. When an operator is considering the application of a RPM/DPR treatment that does impart some permeability reduction to oil flow in the treated reservoir volume, he or she must factor this into the treatment design and the expected treatment performance.

The application of a non-ideal RPM/DPR treatment could be an attractive business venture for an operator, for example, for a treatment that reduced oil production by only $5 \%$, but reduced water production by $90 \%$.

Additionally, an ideal RPM/DPR treatment does not wash out with time and is not back produced in the field setting. 


\section{When and Where Applicable}

Matrix-Reservoir-Rock Radial-Flow Vertical Wells. In this section, we assume gravity effects are negligible and assume the application of an "ideal" RPM/DPR technology. An ideal RPM/DPR treatment imparts no permeability reduction to oil or gas flow, but imparts a relatively large permeability reduction to water flow in the treated reservoir volume. This section of the chapter is limited to discussion of RPM/DPR treatments of unfractured production wells (radial flow through matrix rock or sand).

Fully Drawn Down Wells: If a well is fully drawn down before a treatment, we normally expect it to remain so after the treatment (so long as the treatment is not applied over fracture pressure). We assume here that the production conditions and equipment are the same before and after the treatment. The following discussion is specifically targeted at oil-producing wells, but the same general arguments also hold for gas-producing wells.

When a well is fully drawn down, the application of a RPM/DPR treatment (alone) provides no opportunity to increase the post-treatment oil-production rate.

Long-Term WSO: In this subsection, we discuss when and where long-term (months to years) RPM/DPR treatments can be successfully applied to production wells in matrix rock reservoirs where flow is radial into the wellbore. Alternatively by "long-term," we mean the target longevity of the treatment life is the economic life of the treated well - the ideal longevity goal for any highly effective and durable WSO treatment. By "successful" WSO treatments, we mean treatments that reduce the water production rate, while not simultaneously reducing the pretreatment oil-production rate.

- Single oil-producing zone (geological strata or formation): $R P M / D P R$ treatments are not applicable. RPM/DPR treatments are of little practical value (for providing long-term [e.g., years of] water shutoff) when applied to a single zone (relatively homogeneous) reservoir that is producing at a high water cut. As shown in Fig. 15, this is because after the treated well is put back on production, a relative-permeability water block will form just beyond the outermost penetration of the treatment. ${ }^{38,39,57,58,63,64}$

The relative-permeability water block occurs because after the RPM/DPR treatment, water and oil in the far wellbore region continue to flow to the well at the originally produced water/oil ratio. When this oil/water fluid stream reaches the outer radial penetration of the treatment, the water flow is impeded, whereas no permeability reduction and impediment (for an ideal treatment) is encountered by the oil flow. Thus, with time, the water saturation builds up just beyond the treatment material (polymer or gel). As the water saturation builds up, the relative permeability to oil flow is reduced. As a result, the oil permeability is also reduced in this volume. In this chapter, the term "water-block problem" refers to this treatment-induced reduced oil relative permeability and the consequential reduction in oil productivity from the treated zone.

The best that anyone can do in this single oil-producing-zone situation over the long term is to end up with the final/equilibrium water cut being the same as the pre-treatment water cut, but the well producing at lower production rate. ${ }^{38,39,57,58,63,64}$ This is a lose/lose result. The 
WOR ratio is not reduced, and the oil-production rate is reduced. In the proceeding discussion, the single zone was considered to be homogeneous; however, from a practical point of view, this argument normally still holds if the single oil-producing zone is mildly or somewhat heterogeneous. Stated another way, RPM/DPR treatments are of no value for promoting long-term WSO within any single, isolated oil-producing zone that is nearly watered out.

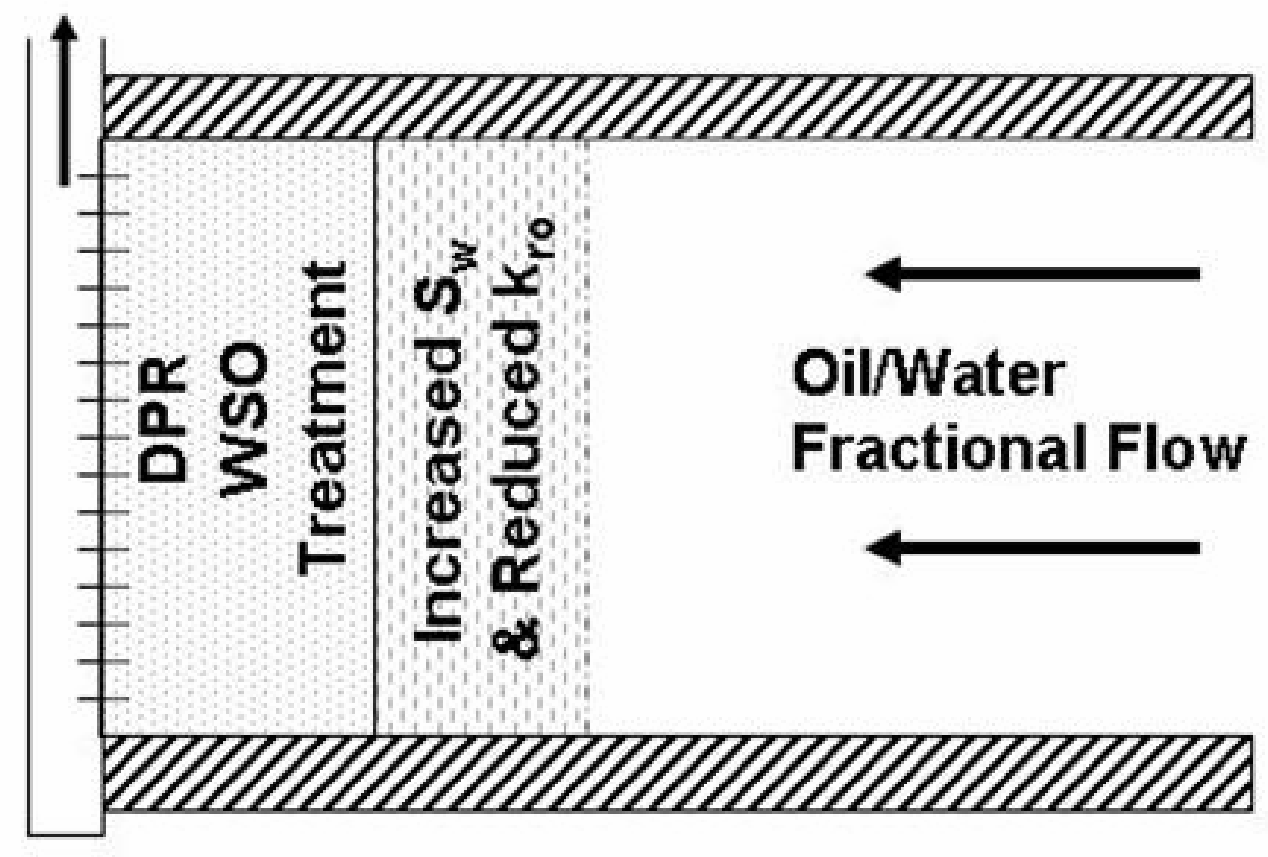

Fig. 15-DPR treatment in a single strata producing at a fixed fractional flow.

- Multiple producing intervals in the reservoir:

$>$ Crossflow exists between reservoir zones/strata: $R P M / D P R$ treatments are not applicable. ${ }^{46}$ By crossflow, we mean that the various reservoir geological strata within the producing reservoir are in vertical pressure and fluid communication (e.g., continuous impermeable shale barriers do not exist between the reservoir geological strata). Stated another way, a finite $k_{v}$ exists across the reservoir intervals in question. In view of the previous discussion under the "Single oil-producing zone" bullet item, what is a little less obvious is that for the same basic reason when producing from matrix rock reservoirs in the radial-flow mode, RPM/DPR treatments are not effective at promoting long-term water shutoff/reduction anytime crossflow exists between the oil- and water-producing zones. This is shown in Figs. 16 and 17. The phenomenon depicted in Figs. 16 and 17 will also occur if the water-producing interval overlies the oil-producing interval. The situation depicted in Figs. 16 and 17 is not representative of water coning because: (1) the lower zone in the figures is implicitly of much higher permeability than the upper zone, and (2) operators do not normally perforate below the oil/water contact. 


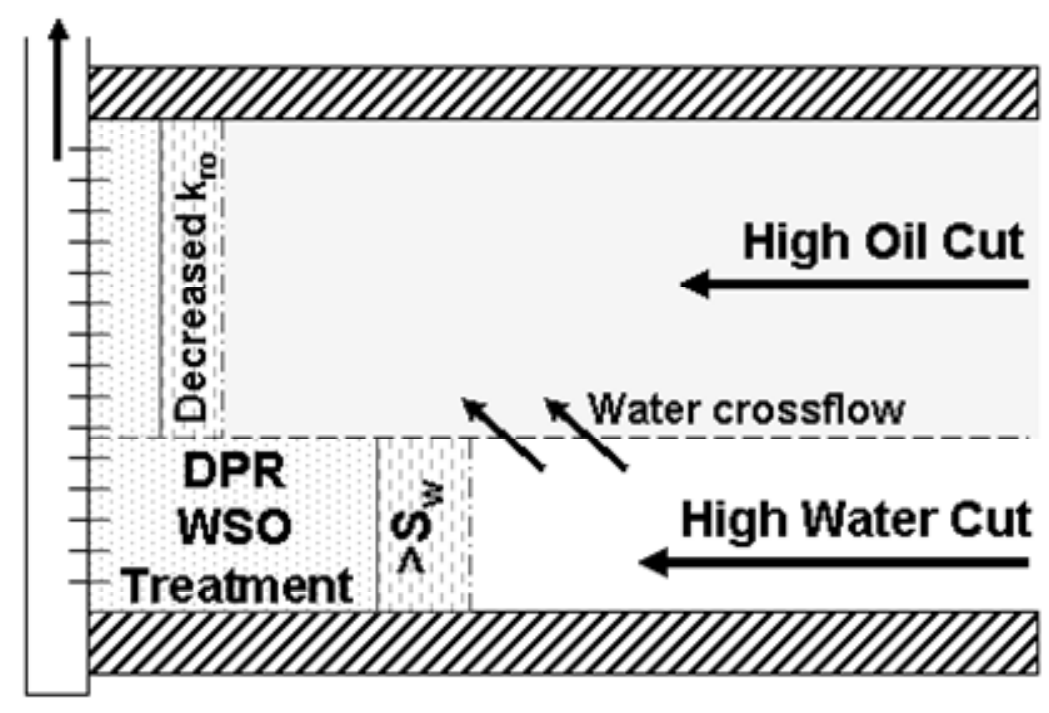

Fig. 16-DPR treatment in a reservoir with crossflow.

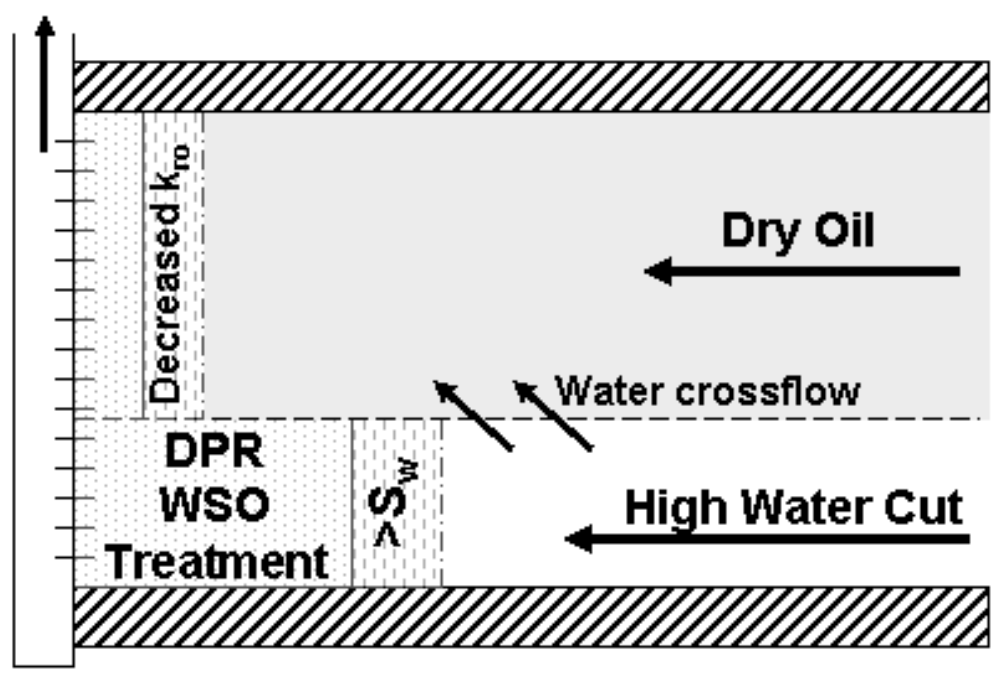

Fig. 17-DPR treatment in a reservoir having a dry-oil producing strata with crossflow.

$>$ Crossflow between reservoir zones/strata does not exist:

- Oil zone(s) is producing at $100 \%$ oil cut (dry oil): $R P M / D P R$ treatments are applicable. As shown in Fig. 18, this favorable result occurs because no water-block problem forms in the oil-producing zone(s). This is a type of excessive waterproduction problem that is amenable to successful RPM/DPR treatments (for wells that are fully drawn down). ${ }^{59-61,63}$ To maintain this favorable result, the oil-producing zone(s) must continue to produce dry oil for the economic life of the treatment. 


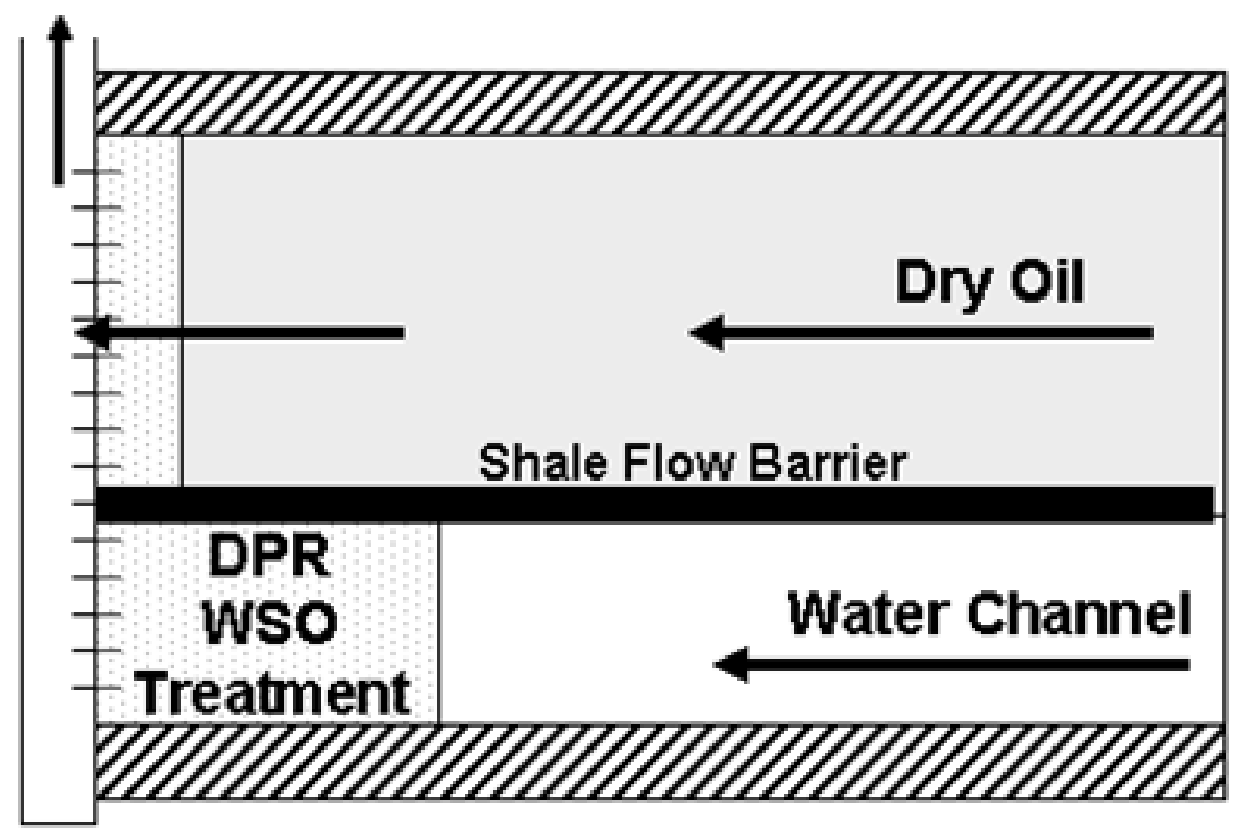

Fig. 18-DPR treatment in a reservoir with no crossflow.

- Oil zone(s) producing at a finite (intermediate) water cut: Long-term RPM/DPR treatments are not applicable. This problem degenerates to the problem of a series of isolated oil-producing zones producing at a finite water cut and the associated geltreatment-induced water-block problem (as discussed previously). As mentioned above, any treatment cannot change the steady state fractional flow from a given zone. If a zone produces at an intermediate water cut (e.g., 20\% water and $80 \%$ oil) before treatment, the water cut must have the same value after treatment. ${ }^{6}$ Thus, if polymer or gel enters and causes a two-fold loss of water productivity from this zone, it must also cause a two-fold loss of oil productivity. On the other hand, circumstances may exist where some loss of oil productivity may be acceptable if dramatic reductions in productivity can be achieved from other prolific waterproducing zones (e.g., the bottom zone in Fig. 18).

- Water coning through unfractured matrix rock: RPM/DPR treatments are generally not applicable. For justification of this assertion, see Refs. 6, 8, 59, and 60. Except under rare circumstances, ${ }^{6,8}$ RPM/DPR treatments can only delay (normally for a relatively short period of time) the water from coning around the emplaced treatment.

Short-Term WSO: In this subsection, we discuss when and where short-term ("transient") $\mathrm{RPM} / \mathrm{DPR}$ treatments can be applied with some success to production wells in matrix rock (unfractured) reservoirs where radial flow is occurring. By "short-term", we mean treatments that promote WSO for hours to a month or two (but often hours to days). 
- Single (homogeneous or nearly homogeneous) oil-producing zone: Short-term RPM/DPR treatments can possibly be applicable. Short-term RPM/DPR treatments, in theory, can be applied with some success ${ }^{39}$ because immediately after treatment placement and during initial post-treatment production, oil can "readily" pass through the gel-treated matrix rock volume (for an ideal treatment), while simultaneously water production is significantly impaired. However, the post-treatment production rate in the oil zones will decrease to an equilibrium level as the water-block is established at a point just beyond the outer radial penetration of the treatment material. As a result, favorable long-term WSO will not result. The economics of applying RPM/DPR treatments that impart short-term/transient WSO are often marginal, and these are relatively high risk WSO treatments, where each treatment needs to be custom designed, evaluated, and engineered. The treatment design and expected performance needs to be carefully evaluated in terms of both technical and economic considerations. Refer to Fig. 15 when considering this particular problem.

- Crossflow between reservoir zones/strata does not exist:

$>$ Oil zone(s) producing at 100\% oil cut: Short-term RPM/DPR treatments are applicable. However in this case, the RPM/DPR treatment will also promote long-term WSO which is a more favorable outcome.

$>$ Oil zone(s) producing at a finite water cut: Short-term RPM/DPR treatments can possibly be applicable. This problem degenerates to the problem of a series of isolated oilproducing zones producing at a finite water cut and to a version of the problem described in the first bullet item of this subsection. That is, a series of isolated producing intervals, as depicted in Fig. 15, where the zones overlay one and other.

- Crossflow exists between the oil- and water-producing zones with the oil-producing zone(s) either producing at $100 \%$ oil cut or at a finite water cut: Short-term RPM/DPR treatments can possibly be applicable. This is because following the application of a RPM/DPR treatment, it takes a finite period of time for the water block to establish itself just outside of the treatment penetration radius in the oil-producing zone(s). Beginning with first posttreatment production, the oil production rate in the oil-producing zones will be decreasing to an equilibrium level as the water block is established. This can be seen by carefully studying Figs. 16 and 17. The economics of applying RPM/DPR treatments that impart shortterm/transient WSO are often marginal, and these are relatively high-risk WSO treatments. Each of these treatments needs to be custom designed and engineered. The treatment design and expected performance needs to be carefully evaluated in terms of both technical and economic considerations.

Wells Not Fully Drawn Down: Production wells that are not fully drawn down before application of a RPM/DPR treatment often experience increased drawdown pressure after application of the WSO treatment. The ability to increase drawdown pressure provides the means to possibly increase the oil-production rate after the WSO treatment. In order for a RPM/DPR treatment to increase the oil-production rate, the treatment must "significantly" increase the drawdown pressure. By significantly increasing the post-treatment drawdown pressure, we mean that in the Darcy radial-flow equation, the magnitude of the post-treatment drawdown pressure increase exceeds the magnitude of the loss of the overall effective permeability to oil flow. That is, oil permeability lost due to treatment-induced damage to oil flow in the treated reservoir volume and/or any treatment-induced water-block problem(s). To generate an increased oilproduction rate, the effect of the increased drawdown pressure must exceed the effect of the loss 
in well's productivity due to treatment-induced loss of oil permeability and due to the formation of any treatment-induced water block.

This can be quantitatively seen by considering Darcy's radial-flow equation,

$q_{o}=\left(\Delta p k_{o}\right)\left(h /\left[141.2 \mu \ln \left\{r_{e} / r_{w}\right\}\right]\right)$

where $q_{o}$ is the oil production rate in BPD, $\Delta p$ is differential pressure in psi, $k_{o}$ is the effective permeability (in md) for oil flow from the entire producing interval, $h$ is the total height of the producing interval, $\mu$ is oil viscosity in $\mathrm{cp}, r_{e}$ is the external drainage radius in $\mathrm{ft}$, and $r_{w}$ is the wellbore radius in ft. In Eq. 7, $\Delta p$ and $k_{o}$ are the two key variables of interest. The other variables on the right side of Eq. 7 are fixed. Oil production will only increase if the magnitude of the increased drawdown pressure exceeds the magnitude of the loss of oil flow capacity caused by the treatment.

Viewed in another way, treatment-induced increased drawdown pressure in a treated well provides a countervailing phenomenon to help, or possibly fully, offset RPM/DPR treatmentinduced damage to oil permeability in the treated reservoir volume (for a "non-ideal" WSO treatment) and/or treatment-induced water-block problems.

Single oil-producing zone: RPM/DPR treatments are not applicable. Under all post-treatment drawdown-pressure conditions, single (homogeneous or nearly homogeneous) oil-producing zones cannot be successfully treated with long-term RPM/DPR treatments due to the water-block problem (discussed previously) that occurs outside the outer radial penetration of the WSO treatment.

Multiple zones with, or without, crossflow, and with, or without, the oil zone(s) producing at 100\% oil cut: RPM/DPR treatments can possibly be applicable. The technical and economic applicability, in this instance, of a RPM/DPR treatment to wells that are now fully drawn down must be evaluated on a well-by-well basis.

Treatments Exploiting Gravity Effects. RPM/DPR treatments that exploit gravity effects in matrix rock reservoirs may be beyond the primary scope of this chapter. However, there have been a few isolated instances where aqueous gel RPM/DPR treatments were based on the exploitation of the gravity concept. Fig. 19 shows how capitalizing on gravity might be exploited. For the sake of completeness, this subject is briefly covered in this subsection.

We emphasize the difference between the case considered here (Fig. 19) and conventional 3D coning. In normal 3D coning in matrix rock, the absolute permeability of the underlying aquifer is typically about the same as in the hydrocarbon zone. For the case in Fig. 19, the water zone is much more permeable than the overlying hydrocarbon zone.

In order for a RPM/DPR treatment to exploit gravity effects, the water-producing interval must be located at the bottom of the producing interval and there must be good pressure and fluid communication (good $k_{v}$ ) between the oil and water producing zones. 


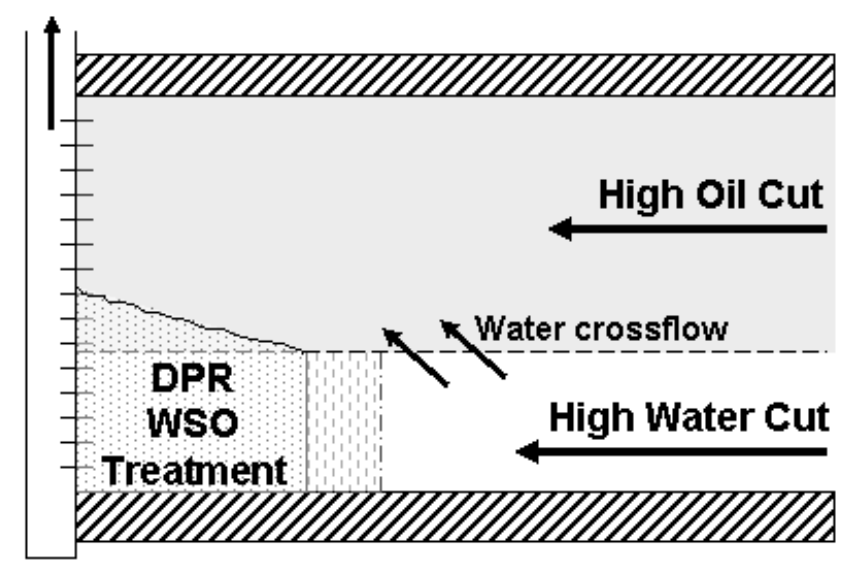

Fig. 19-A gravity-exploiting DPR treatment.

The successful application of RPM/DPR treatments that exploit gravity effects are favored by:

- High permeability producing intervals

- Long gel onset times

- Low oil viscosity

- High density contrast between the treatment fluid and the oil

- Thick hydrocarbon-producing zones

However, in this instance, the use of an appropriate classical "total shutoff" WSO gel would work just as well, if not better. Also, a plug back operation (e.g., sand back plug) within the wellbore is operationally less complex, usually less costly for this application, and often nearly as effective.

\section{Matrix-Reservoir-Rock Radial-Flow Horizontal Wells.}

Coning via Matrix Rock: RPM/DPR long-term WSO treatments are not applicable. Such WSO treatments are not applicable to water coning into a horizontal well for basically the same set of general reasons ${ }^{6,8,59,60}$ that WSO treatments cannot be effectively applied to promote long-term WSO when the excessive water production is coning into a vertical well producing from a matrix rock reservoir. That is, any such WSO treatment will only delay the water coning.

\section{Fractured Wells.}

Hydraulically Fractured Production Wells. When production wells are hydraulically fractured, the fracture often unintentionally breaks into water zones, causing substantially increased water production. RPM/DPR gel WSO treatments (involving robust, relatively strong, and pore-filling polymer gels) have significant potential to correct this problem. These gel WSO treatments rely on the ability of these gels to be placed in the rock matrix adjacent to the fractures and to reduce permeability to water flow much more than that to hydrocarbon flow (DPR). An engineeringbased method has been developed for designing and sizing gelant treatments in hydraulically fractured production wells. 7,13 
These gel WSO treatments permit the use of: (1) gel that reduces the permeability to oil flow significantly (greater than a factor of 2) within the reservoir volume where the gel is placed, and (2) pore-filling, robust, relatively strong, and more classical polymer gels.

In these matrix rock treatments, the gelant fluid (gel fluid in which "no" gelation has yet occurred) flows along the fracture and leaks off a short, predictable distance into the matrix rock of all the zones (water, oil, gas). Success for such a treatment requires that the gel reduce permeability to water much more than that to hydrocarbon (oil or gas) in the treated matrix rock (Fig. 20). The ability of the gel to reduce water entry into the fracture is determined by the product of gelant leakoff distance (from the fracture face) and the residual resistance factor (permeability reduction factor) provided by the gel. For example, consider the case where the gelant leaks off $0.2 \mathrm{ft}$ into both water and oil zones, and in the gel-contacted rock, permeabilities to water and oil are reduced by factors of 50,000 and 50, respectively. In this case, the gel only adds, effectively, the equivalent of 10 feet of additional rock that the oil must flow through to enter the fracture (i.e., $0.2 \mathrm{ft} \times 50$ ). In contrast, for the water zone, the water must flow through the equivalent of $10,000 \mathrm{ft}$ of additional rock to enter the fracture (i.e., $0.2 \mathrm{ft} \times 50,000$ ). Thus, in this circumstance, the gel can substantially reduce water production without significantly affecting oil productivity.

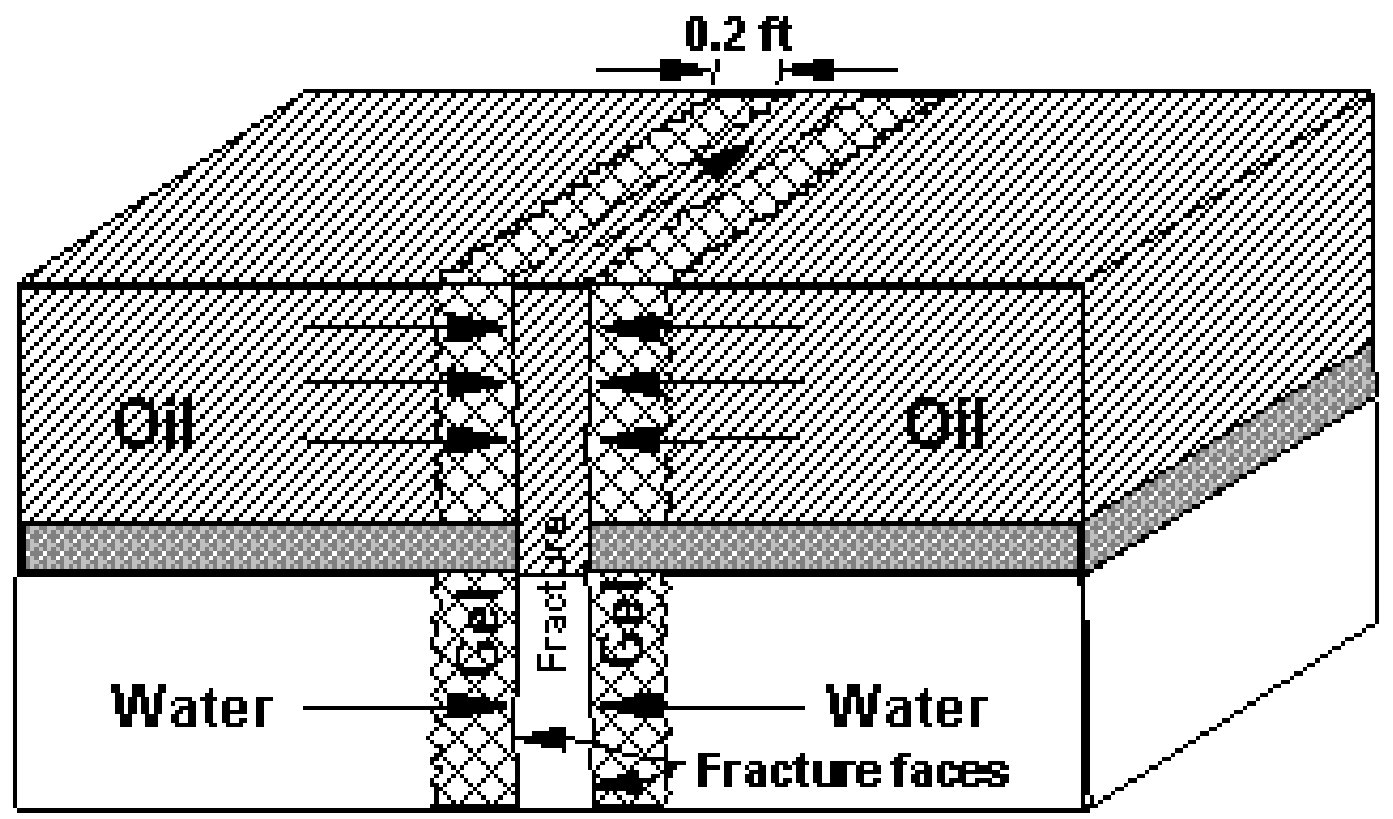

\section{Equivalent resistance to flow added by the gel In oil zone: 0.2 ft $x \mathbf{5 0}=\mathbf{1 0} \mathrm{ft}$. In water zone: $0.2 \mathrm{ft} x \mathbf{5 0 , 0 0 0}=10,000 \mathrm{ft}$.}

Fig. 20-Use of DPR to inhibit water entry into a fracture or fracture system 
In this method, fluid entry into the fracture is controlled by the gel in rock next to the fracture. ${ }^{7,13}$ Ideally, fracture conductivity should not be reduced significantly, since it allows a conductive path for hydrocarbon flow into the wellbore. To some extent, gravity segregation of the gelant (between placement and gelation) will mitigate damage to the fracture when the excessive water production originates from an underlying aquifer. However, to minimize fracture damage, an oil or water post-flush could be used to displace gelant from the fracture.

From a rigorous viewpoint, the method assumes that impermeable barriers (e.g., shale or calcite) separate adjacent zones. ${ }^{7}$ However, the method should frequently provide acceptable results even if crossflow can occur between the water bearing and oil bearing zones. For example, consider the case where oil lies on top of water in a single formation (i.e., a common situation where coning becomes a problem). Previous work ${ }^{6,8}$ showed that gravity alone can retard water influx into oil zones much more effectively when the water must "cusp" to a linear pressure sink (i.e., a vertical fracture or a horizontal well) than when the water "cones" to a point pressure sink (i.e., a partially penetrating vertical well). For the type of gel treatment that we are proposing for application in hydraulic fractures, in many cases, gravity may be sufficient to minimize water invasion into the hydrocarbon zone of a single formation. Of course, the degree of water invasion (coning) into hydrocarbon zones increases with increased production rate, pressure drawdown, vertical permeability, and hydrocarbon viscosity, and decreases with increased waterhydrocarbon density difference and oil column thickness. ${ }^{6,8}$ If water invades too far into the hydrocarbon zone, a water block could form that reduces hydrocarbon productivity.

To use this procedure to reduce water production from a hydraulic fracture, field data are needed, coupled with results from two simple laboratory experiments. ${ }^{7}$ The needed field data include: (1) fluid production rates before the gel treatment, (2) downhole static and flowing pressures before the gel treatment, (3) permeabilities, porosities, and thickness of the relevant zones, (4) water and oil viscosities at reservoir temperature, and (5) well spacing or distance between wells. These parameters are often available during conventional gel treatments. The downhole pressure drops are critically important for this method. They must be reasonably current and measured specifically for the well to be treated.

Use of the procedure also requires oil and water residual resistance factors from laboratory core experiments. $^{7,13}$ These experiments must be conducted using the gelant, oil, brine, rock, and temperature that are representative of the intended application. In the absence of laboratory oil and water residual resistance factors, the model can use field data to back-calculate these values in situ after a gel treatment. This information may be useful when designing similar treatments in nearby wells. For cases where residual resistance factors are calculated from field data, three parameters (from a similar, previous gelant treatment) are required in addition to the five items listed in the previous paragraph. These three parameters are: (1) fluid production rates after the gel treatment, (2) accurate downhole static and flowing pressures after the gel treatment, and (3) the volume of gelant injected.

Naturally Fractured Production Wells. The previously discussed concepts have also been applied to applications in more complex naturally fractured vertical wells where fractures connect to a water source, although additional work is needed is this area., 95 
Horizontal Wells with Fractures that Lead to an Aquifer. Horizontal wells often intersect fractures that lead to an aquifer. Field cases exist where a partially formed or fully formed (mature) gel of the classical type was extruded down the length of the well and into a fracture. ${ }^{66,67}$ Classical fracture-problem gels are relatively "strong" and total-fluid-flow-shutoff gels. See Ref. 64 for a discussion of strong gel application for this excessive water-production problem. Because the formed gel cannot enter the porous rock, it causes no significant damage to hydrocarbon-productive zones. However, when extruding through the water-producing fracture, the gel dehydrates (concentrates). When the well is returned to production, the concentrated gel remains in the fracture (if the treatment is designed correctly) and prevents water from entering the well from the underlying aquifer.

An alternative to the above approach could exploit DPR. Instead of a formed gel, gelant (gel fluid in which "no" gelation has yet occurred) could flow down the horizontal well and into the offending fracture, leaking off into porous rock during the entire placement procedure. When the gelant sets up in the porous rock next to the fracture within the aquifer, the gel effectively encapsulates the fracture and greatly restricts water entry. In contrast, although gelant has entered hydrocarbon-productive zones along the well, the DPR effect (if properly designed and sized) could allow hydrocarbon to enter the well with limited loss of productivity.

\section{Guidelines}

The following provides guidelines as to when and where RPM/DPR treatments can be successfully applied, especially with regard to reservoir, geological, and production conditions.

The following guidelines assume the application of an "ideal" RPM/DPR treatment, where the treatment does not impart any significant reduction to oil permeability in the treated reservoir volume. If the treatment does reduce the permeability to oil flow in the treated reservoir volume, this must be factored in separately to the following guidelines.

In the following Guidelines (Table 5) under the Applicable column, "Depends" means depending on whether the magnitude of the post-treatment drawdown pressure increase exceeds the magnitude of the loss of the overall effective permeability to oil flow occurring from the treated well. 
Table 5-Guidelines for RPM/DPR treatments.

\section{Guidelines - When \& Where Ideal RPM/DPR Treatments Are Applicable}

- Matrix-reservoir-rock radial-flow vertical wells

\section{Applicable}

$>$ Fully drawn down wells

- Long-term WSO

A Single homogeneous oil-producing

No zone

A Multiple zones

- Cross flow exists

- No crossflow exists

- Oil zone(s) producing at $100 \%$ oil cut

- Oil zone(s) producing at a finite-water cut

- Short-term WSO

A Single homogeneous oil-producing zone

ᄉ Multiple zones

\section{Possibly \\ Possibly}

Wells not fully drawn down

- Long-term WSO

A Single oil-producing zone

No

A Multiple zones

- Oil zone(s) producing at $100 \%$ oil cut

- Oil zone(s) producing at a finite water cut

- Short-term WSO

A Single homogeneous oil-producing zone

ᄉ Multiple zones

No

Yes

No

Yes

Depends

Possibly

Possibly

- Matrix-reservoir-rock radial-flow horizontal wells (long-term WSO)

$>$ Water coning

No

- Fractured wells

$>$ Vertical wells

- Hydraulic fracture extending into a fracture

Yes

- Single natural-fracture problem

Yes

- Limited natural-fracture-network problem

Yes

- Extensive natural-fracture-network problem

$>$ Horizontal wells

- Fracture(s) connected to an aquifer

\section{Challenging}

Yes 


\section{Treatment Limitations and Potential Pitfalls}

This section will briefly discuss a series of limitations and potential pitfalls that often apply to RPM/DPR treatments.

Treatments for Matrix-Reservoir-Rock Radial Flow Wells. Presently available RPM/DPR treatments for application to wells in matrix rock reservoirs producing under radial flow conditions usually involve the use of water-soluble polymers alone or relatively weak polymer gels. The following treatment limitations and potential pitfalls pertain to RPM/DPR treatments that are to be applied to wells of matrix-reservoir-rock reservoirs producing under radial flow conditions.

Oil Permeability Always Reduced: To date, all known commercial RPM/DPR treatment technologies for this application reduce the permeability to oil flow to some degree in the treated reservoir volume. $^{40}$ The goal of these WSO treatments should be to not reduce the permeability to oil flow by a factor exceeding two. $22,39,44$

However, for the sake of completeness, it should be noted that Ref. 35 discusses a laboratory study of gel and polymer-alone use for WSO purposes in gas wells. In this chapter during certain instances, the gas permeability was observed to be fully maintained, or to increase somewhat, following application of the WSO treatment. However, these laboratory studies were not of just classical RPM/DPR treatments, but treatments that additionally involved the sequential injection of gas slugs during the WSO treatment fluid placement.

Permeability Dependence: For adsorbing polymers and weak gels, residual resistance factors increase with decreasing permeability. ${ }^{21,14,15,18-20}$ In other words, these materials damage lowpermeability rock more than high-permeability rock. Depending on the magnitude of this effect, these polymers and gels can harm production flow profiles in wells. ${ }^{6,13,17}$

This phenomenon is counterproductive for RPM/DPR treatments because adsorbing polymers and weak gels often reduce the flow capacity more in the low-permeability oil-producing zones than in the high-permeability water-producing zones/channels. This is the opposite of what is desired of a WSO treatment.

Limited Permeability Range of Applicability: All presently available RPM/DPR treatment technologies have a limited range of absolute permeability over which they are applicable. ${ }^{11,60,63}$ This is especially true for the polymer-alone RPM/DPR technologies. Since operators often underestimate the permeability of their water-producing reservoir channels, ${ }^{68}$ this has proven historically to be an especially acute problem and the explanation for many field failures of RPM/DPR treatments. Thus, it is critical that the operator correctly estimate the permeability of his water-producing channels and/or reservoir water flow paths if he or she is considering the application of a RPM/DPR treatment.

Erratic Performance: The performance of currently available RPM/DPR treatment technologies, in both the laboratory and field setting, has proven to be quite erratic. ${ }^{11,22,63}$ This is true even for the same treatment applied two or more times under "identical" conditions in the "same" core 
material in the laboratory or the same treatment applied in the same field to highly similar wells. Erratic behavior and performance is more acute for those RPM/DPR treatments that are meant to promote short-term/transient WSO. This limitation reduces the attractiveness of RPM/DPR treatments and increases the uncertainty when applying such treatments in an oil or gas field.

Variability of residual resistance factors may be an inherent flaw for adsorbed polymers and weak gels. Permeability reduction by adsorbed polymers can be strongly influenced by rock mineralogy. In turn, rock mineralogy typically exhibits significant variations locally within a porous medium. Consequently, these mineralogical variations could lead to wide variations in performance for adsorbing polymers.

Weak gels are typically suspensions of gel particles - not a continuous three-dimensional gel structiure. $^{12,14,15}$ These particle suspensions have a particle size distribution-they are not monodisperse. Pores and pore throats within a rock also have a size distribution. Since the particles reduce permeability by lodging in pore throats, the ratio of particle size to pore-throat size is important in determining residual resistance factors for these suspensions. Variations in particle size distribution (especially resulting from unknown or uncontrolled particle generation) and variations in pore-throat size distribution (resulting from normal geologic processes) may cause wide variations in WSO performance for weak gels.

Back Production and Washout: Another significant limitation and potential concern and pitfall for RPM/DPR treatments is the tendency of the emplaced WSO material (for many such treatment technologies) to be back produced and washout, especially when placed in the highdifferential-pressure region adjacent to a radial flow production well. In addition, this is especially true for RPM/DPR treatment technologies that are based on the use of water-soluble polymers alone, where the WSO mechanism involves the adsorption of the polymer onto pore walls and/or in pore-throat constrictions. This can also be a serious problem for RPM/DPR technologies that are based on the use of weak polymer gels. Use of pore-filling RPM/DPR gels may mitigate this problem. ${ }^{44}$

Slow Restoration of Oil Permeability: The slow clean up (restoration) of oil permeability in treated matrix porous media, ${ }^{22}$ as is exhibited by numerous RPM/DPR systems, could possibly prove to be problematic and a limitation.

A simple mobility-ratio model was developed to predict cleanup times for both fractured and unfractured wells after a gel treatment. ${ }^{22}$ The time to restore productivity to a gel-treated oil zone: (1) was similar for radial versus linear flow, (2) varied with the cube of distance of gel penetration, (3) varied inversely with pressure drawdown, (4) varied inversely with the $k_{w}$ at $S_{o r}$ in the gel-treated region, and (5) was not sensitive to the final $k_{o}$ at $S_{w r}$. Although $k_{o}$ at $S_{w r}$ (after gel placement) had no effect on the cleanup time, it strongly affected how much of the original oil productivity was ultimately regained.

Treatments for Fractured Wells. To follow is a brief listing and discussion of limitations of $\mathrm{RPM} / \mathrm{DPR}$ treatments where the treatment material is placed in the matrix rock that is adjacent to the treated fractures. 
Size of the Fracture System: RPM/DPR treatments can be used effectively to treat water production that emanates from finite-volume hydraulic fractures that extend out of zone into an aquifer or water strata. ${ }^{7}$ Also, these WSO treatments have been successfully applied to natural fracture networks of limited extent and size. ${ }^{9}$

However, the successful application of these WSO treatments to extensive fracture networks is more challenging for two reasons. First, as further discussed in the next section, obtaining a uniform depth of gel placement into the matrix reservoir rock (adjacent to the treated fracture) becomes more challenging as the size of the fracture network increases. Second, for RPM/DPR treatments that are to be placed to any significant depth in the matrix rock, the treatment volume and cost may become prohibitive as the size of the fracture network increases beyond some critical value.

Obtaining Uniform Depth of Treatment Placement: As the size of the fracture(s) or fracture network increases, it becomes more difficult to obtain uniform depth of placement of the $\mathrm{RPM} / \mathrm{DPR}$ treatment material into the matrix rock that is adjacent to the fracture(s). There are two major factors contributing to this problem. First, for large volume treatments that take a long time to inject (many hours to days), the fracture faces nearer the wellbore experience more contact time with the injected treatment fluid, and thus will experience deeper penetration of the treatment fluid into the matrix rock. Second, and especially in fractures having significant aperture widths (e.g., greater or equal to $1 \mathrm{~mm}$ ), significant gravity effects may occur during aqueous treatment-fluid placement where the aqueous treatment fluid may segregate to the lower portion of the fracture. Of course, this could prove to be an advantage if water is being produced from the lower portion of the fracture, and oil is being produced from the upper portion.

Question of the Water Source: When considering the application of a RPM/DPR treatment involving placing the gel into the matrix rock that is adjacent to the water-producing fracture(s), the water source is an important issue. If water is being co-produced with oil from the matrix reservoir rock into a fracture or fracture system, this is a good gel WSO scheme. However, if the majority of the oil is produced into the fracture or fracture system from the matrix reservoir rock, but the majority of the water is produced through the fracture from a source far from the wellbore, this is not a good gel WSO scheme.

\section{Discussion}

RPM/DPR Treatments of Gas Wells. Although this chapter has implicitly emphasized the application of RPM/DPR treatments to oil production wells, these treatments are also very applicable to gas production wells. Ref. 2 describes a number of gels that impart disproportionately large permeability reductions to water flow, relative to oil and gas flow. We feel that the application of RPM/DPR treatments is nearly equally applicable to both oil and gas production wells and that the application of RPM/DPR treatments to gas production wells has been, to date, under exploited.

Determine or Deduce the Water-Production Problem. It is imperative that an operator correctly deduce the source and nature of the excessive and unnecessary water-production problem before considering, designing, and implementing a RPM/DPR treatment. 
Need for Custom Engineering. If a RPM/DPR treatment is to be applied in a new field for the first time, the WSO treatment must be custom designed and engineered. Under these circumstances, RPM/DPR jobs are not routine, low-risk, "cookie cutter" treatments. Operators, who are not experienced with the application of RPM/DPR treatments, should proceed with caution when considering applying such a treatment.

Fractional Flow. For treatments that are to be placed in fully drawn down radial-flow wells of matrix rock reservoirs, oil/water fractional flow in any given geological strata (zone) is a serious challenge to presently available RPM/DPR treatments that are intended to promote long-term WSO. ${ }^{11}$ This is because of the water-block problem ${ }^{38,39,57,58,63,64}$ that occurs just beyond the outer radial penetration of the RPM/DPR treatment. After treatment placement, the fractional flow in the far-wellbore region remains unchanged. At the outer radial penetration of the RPM/DPR treatment, water flow is impeded, water saturation builds up at this point, the relative permeability to oil is reduced, and oil production is thus impeded.

As a result, RPM/DPR treatments that are intended to promote long-term WSO are not applicable to a single-zone reservoir.

In the case of a multi-zoned reservoir, where the water-producing zone is not in fluid and pressure communication with the other zones and the well is fully drawn down, RPM/DPR treatments will cause a loss in oil-production rate from zones that have a finite fractional flow (i.e., not $100 \%$ oil cut) due to the water-block problem. ${ }^{38,39,57,58,63,64}$ In this case, RPM/DPR treatments are of dubious value unless in the case of wells that are not fully drawn down, the post-treatment drawdown pressure can be substantially increased and incremental oil production can possibly be obtained from the other strata resulting from increased drawdown pressure.

For a zone that is producing at a high water cut and fluid crossflow can occur into adjacent zones, the resultant water-block problem will cause detrimental water crossflow as depicted in Fig. 16.

Water Crossflow. After treating matrix rock reservoirs with a RPM/DPR long-term WSO treatment and where water crossflow can occur between the water- and oil-producing zones, water crossflow into oil-producing strata can be problematic, especially when the drawdown pressure on the producing formation after the treatment is not, or cannot be, significantly increased.

As can be seen by studying Fig. 17, this can prove to be especially troublesome when the oil cut is $100 \%$ in the oil-producing zone. In this case, water crossflow creates a detrimental water-block in the oil-producing zone.

Drawdown Pressure. For unfractured production wells (i.e., radial flow from matrix rock) that are not fully drawn down before a treatment, the magnitude of the increase in the drawdown pressure after a RPM/DPR long-term WSO treatment has major implications. If the posttreatment drawdown pressure is not significantly increased, then unless oil is produced at $100 \%$ oil cut (dry oil) from isolated strata, RPM/DPR treatments are unable to promote increased oil 
production rates and/or substantially compensate for any treatment-induced loss in oil productivity.

Stated another way, any treatment-promoted increased oil production rate is proportional to the increase in the post-treatment drawdown pressure (beyond a critical value that is related to the treatment-induced loss in oil productivity).

The implication of this observation is that applying RPM/DPR treatments to wells that are not fully drawn down holds the possibility (if a whole set of conditions can be met) to increase the oil production rate following the treatment. On the other hand, if the treated wells are initially fully drawn down and all the oil production is produced at finite fractional flow, some oil production rate will always be lost when applying a RPM/DPR treatment.

For wells that are not fully drawn down, post-treatment increased drawdown pressure provides a countervailing phenomenon to help, or possibly, fully mitigate oil productivity losses caused by oil permeability damage in the treated reservoir volume and/or by any water-block problems that may be caused by RPM/DPR treatment.

DPR \& Reduction in Water-Producing Rate Do Not Necessarily Correlate. For unfractured production wells, some oilfield personnel have naively believed that the degree of water permeability reduction in the treated reservoir volume will be directly proportional to the degree of reduction in the water production rate that results from a RPM/DPR treatment. This is not true for two reasons.

First, the post-treatment water production rate is dictated by the average overall permeability of the producing interval. After a RPM/DPR long-term WSO treatment, the composite permeability of the producing zone averages two volumes that are in series flow - namely, the bulk of the untreated intermediate- and far-wellbore volume of the producing interval and the near-wellbore volume containing the WSO treatment material. Consequently, the final overall reduction in water permeability of the producing interval is less than the permeability reduction imparted in the near-wellbore-treated reservoir volume. However, because we are dealing with radial flow in these instances, this is often a second order effect and consideration.

Second (and more importantly for unfractured radial flow wells of matrix rock reservoirs that are treated with a RPM/DPR long-term WSO treatment and wells that are fully drawn down before and after the treatment), the following applies. The treatment-induced water-block problem (discussed earlier), which occurs just beyond the outer radial penetration of the WSO treatment material when fractional oil/water flow is occurring in the producing interval, will cause the well's post-treatment reduction in the water production rate to be less than the treatment-induced reduction in water permeability imparted in the treated reservoir volume. This is because the water block causes an overall reduction of the production rate for the treated well. This is not an issue when the oil is being produced at 100\% oil cut (dry oil) from geological strata that are not in fluid and pressure communication with the other strata of the producing interval.

Issue of Possibly Shutting Off Oil Production. Compared to conventional total-fluid-shutoff polymer-gel WSO treatments, some oilfield professionals assert that RPM/DPR treatments 
present little risk of shutting off (totally) oil production if the treatment is inadvertently placed in the oil-producing interval of a vertical well.

If mistakenly placed in the oil-producing strata, numerous conventional polymer-gel WSO treatments are capable of essentially totally shutting off oil production. In some cases, this damage may not be reversible. The concern, in this regard, with RPM/DPR treatments is that a number of oilfield operators infer that such treatments are not likely to damage oil production. There are two reasons that this inference is not necessarily correct. First, many presently available RPM/DPR treatments do reduce oil permeability to a significant extent in the treated matrix rock reservoir volume. Second, and possibly more importantly, the treatment-induced water-block problem ${ }^{38,39,57,58,63,64}$ (described earlier) will often cause reduction of the posttreatment oil-production rate in wells that were initially fully drawn down.

Slow Treatment Cleanup. There is a second water-block problem that can adversely affect the performance of RPM/DPR treatments in unfractured production wells. This problem involves the water of an aqueous-based RPM/DPR treatment that invades the near-wellbore oil-producing zone during treatment injection and placement. ${ }^{39,59,61}$ This problem is especially noticeable when the oil zone is producing dry oil. The problem here is the slow cleanup of the injected treatment water from the oil-producing zone and the associated transient post-treatment reduction in the oil-production rate of the zone in question. As long as the oil-producing zone was at residual water saturation prior to the treatment and there are no clay sensitivity issues, this oilproductivity cleanup and water-block problem usually lasts only for a relatively short duration.

The primary difference between the water-block phenomenon resulting from the injected aqueous treatment fluid and any RPM/DPR-WSO-treatment-induced water-block problems is the cause of the water block. The water block resulting from injecting an aqueous WSO treatment fluid into an oil zone is caused by the water of the injected treatment fluid. The RPM/DPRWSO-treatment-induced water block occurs just beyond the radial penetration of the treatment material. The cleanup mechanism and rules for these two "different" water-block problems are similar.

The cleanup problem involving the injection of the aqueous-based RPM/DPR fluid into an oil zone was not previously discussed in the Treatment Limitations section for two reasons. First, this is a potential problem that must be accounted for during any aqueous-based treatment that is injected for any reason into an oil zone of a matrix rock radial-flow well. Second, this problem can often be effectively managed and mitigated through the use of conventional petroleum engineering practices. Productivity damage caused by relative permeability issues when an aqueous treatment fluid is injected into an oil-producing zone of a matrix rock well can be mitigated, reduced, and/or greatly shortened in duration by either incorporating an appropriate surfactant or mutual solvent into the overall treatment design or injecting a post-treatment stimulation fluid containing an appropriate surfactant or mutual solvent.

Mobile Oil. Any operator considering the application of a RPM/DPR treatment (or any WSO treatment) needs to realize that an oilfield WSO treatment can only be successfully applied to a production well if there is an economically sufficient volume of moveable oil saturation in the 
reservoir surrounding the treated well. This is one of the first considerations that an operator should address when contemplating a WSO treatment.

Wettability. Wettability of the reservoir rock is another factor that an operator needs to consider before application of a RPM/DPR treatment if the WSO mechanism is based on adsorption of polymers or weak gel particles onto the pore wall surfaces of the reservoir rock. RPM/DPR treatments that are based on the adsorption of polymers onto pore walls reportedly often perform less well in oil-wet or intermediate-wet reservoirs. ${ }^{11,54,60,63}$

Deviated Well. Previously in this chapter, we discussed the applicability of RPM/DPR treatments for treating excess water-production problems occurring in vertical and horizontal wells. What happens if the well to be treated is deviated somewhere between vertical and horizontal? If the well is near vertical (within 15 degrees), then it can normally be considered to be a vertical well. Likewise, if the well is near horizontal (within 15 degrees), then it can normally be considered to be a horizontal well. If a deviated well is intermediate to the previously discussed ranges, then good engineering and geological judgment needs to be exercised in how to classify the well and how to design an effective RPM/DPR treatment for such a well.

Historical Performance. The relatively poor performance historically of RPM/DPR treatments has resulted from the following combination of factors:

1. Over expectations of operators regarding RPM/DPR. ${ }^{11}$

2. Over selling of RPM/DPR treatments by oilfield service companies.

3. Failure to recognize the limitations and constraints of RPM/DPR treatments (as discussed in this chapter).

Treatment Risk Factor. For reasons discussed in this chapter, the application of a RPM/DPR treatment for the first time in a new field by an inexperienced operator should not be considered to be a low-risk undertaking.

Treatments Can Be Bullheaded. The primary reason why bullheadable RPM/DPR treatments are of high interest is that they are one of the few options presently available to treat excessive water-production problems in matrix rock reservoirs where mechanical zone isolation is not possible or practical during treatment fluid placement.

Treatment Development \& Exploitation Activity. At the time of the writing of this chapter, laboratory studies, development, and exploitation of RPM/DPR treatments were actively being pursued by numerous petroleum-industry-sponsored R\&D efforts.

What Is Needed. A desired "next generation" matrix rock RPM/DPR (water selective) WSO technology ${ }^{61}$ would have the following properties.

- Greatly reduce (or, more desirable yet, totally eliminate) water permeability during "high" water-cut flow (i.e., provide water residual resistance factors that reliably exceed 100 , and preferably exceed 1,000).

- Totally inactivate (become nonfunctional) during "high" oil-cut flow - at a minimum, consistently provide oil residual resistance factors that are reliably less than two (and preferably near unity). 
- Possess a controllable set point between "low" and "high" water-cut flow where the WSO functionality would be activated.

- Be able to promote effective long-term (i.e., years to decades) WSO.

Extensive efforts are underway to fulfill some of these requirements. Ref. 44 reports several formulations where gels provided water residual resistance factors greater than 2,000 and ultimate oil residual resistance factors of 2 or less. These results provide hope that gels that can be found that successfully and reliably treat either fractured or unfractured production wells without zone isolation.

We also note work by IFP and Delft University in determining permeability reduction values at intermediate water saturations and fractional flows, particularly for adsorbing polymers. ${ }^{21,33,48,52}$ Additional work of this type is needed for other gels (especially pore-filling gels) if extensive RPM/DPR applications are to be applied in hydrocarbon zones that produce at intermediate fractional flows.

\section{Conclusions}

1. When properly designed and executed and when they function downhole as intended, polymer-gel or polymer-alone RPM/DPR treatments can be successfully applied to a limited range of excessive-water-production problems occurring in either oil or gas production wells.

2. When a treatable excessive-water-production problem occurs, RPM/DPR treatments can be applied using bullhead injection (not requiring the use of mechanical zone isolation).

3. When treating an excessive-water-production problem in a matrix rock reservoir where the water is being produced radially into the production well and the well is fully drawn down, the only situation where a RPM/DPR treatment can render long-term WSO, without reducing the pre-treatment hydrocarbon (oil or gas) rate, is when the hydrocarbon and water producing zones are not in fluid and pressure communication and the hydrocarbon zone(s) is producing at $100 \%$ cut (i.e., dry oil) and will continue to do so for the economic life of the WSO treatment.

4. When a multi-zoned unfractured production well (radial-flow through matrix rock) suffers from excessive water production and the well is not fully drawn down prior to the application of a RPM/DPR treatment that is applied for long-term WSO, the oil production rate can possibly be increased if the post-treatment drawdown pressure can be "substantially" increased (as defined in the chapter).

5. RPM/DPR treatments, which provide short-term (transient) decreased WOR, can be, in theory, applied to most production wells (that are not totally watered out) in matrix rock reservoirs where radial flow is occurring. However, each of these treatments must be custom designed and engineered on a well-by-well basis. Furthermore, for most wells and associated excessive water production problems, the performance and the economics of such transient WSO treatments are, at best, marginal.

6. A potentially attractive application of RPM/DPR treatments is the use and placement, in certain instances, of pore-filling and relatively robust gels in the matrix rock that is adjacent to a water-producing fracture(s). 
7. There are a substantial number of limitations and possible pitfalls to the successful application of RPM/DPR treatments, and their application for the first time by an inexperienced operator should not be consider a low-risk undertaking. 


\section{CORRELATING GEL RHEOLOGY WITH BEHAVIOR DURING EXTRUSION}

\section{Introduction}

Excess water production increasingly plagues oil and gas production worldwide. In naturally fractured reservoirs, large volumes of Cr(III)-acetate-HPAM gels have been successfully extruded into place to reduce channeling and excess water production. ${ }^{67,69-71}$ During gel placement in fractures, the pressure gradient for gel extrusion depends strongly on fracture width and gel composition. ${ }^{23,72,73}$ Extrusion experiments directly measure gel properties in fractures, but they are both expensive (for core materials and casting) and time-consuming (two to three days per experiment, with one to two weeks of setup time). In contrast, a single rheology test can be performed at a fraction of the cost (negligible cost for gel materials) and can be completed within several minutes (after one to two days of setup time). Therefore, we investigated whether rheology testing is a more cost-effective and efficient method for predicting gel behavior in fractures.

Earlier researchers ${ }^{74-83}$ employed rheology measurements to characterize gels and gelants that are used for water shutoff. They used dynamic viscometry measurements to monitor the gelation process and study gel properties after gelation. They found that $\mathrm{Cr}$ (III)-acetate-HPAM gels behaved as Bingham plastics under steady shear, exhibiting a linear relationship between shear stress and shear rate above a yield stress. Bird et al. ${ }^{84}$ used a simple force balance to develop an equation that related yield stress to the maximum pressure drawdown that a gel could withstand.

We encountered two apparent discrepancies, however, in using rheometers to predict gel behavior in fractures. First, a simple force balance predicted that the pressure gradient required for gel extrusion between two parallel plates should be inversely proportional to the open width between the plates. ${ }^{84}$ However, when gels extruded through fractures, the pressure gradient was (roughly) inversely proportional to the square of the fracture width. ${ }^{73,85}$ Second, the pressure gradients observed during gel extrusion through the fractures were much greater (by more than 10 times) than those predicted by the simple force balance approach. ${ }^{23,72,73}$

We studied wall-slip effects and first normal stress differences to understand why the simple force balance underestimated the pressure gradients required for gel extrusion. We also used two models based on power-law and shell momentum balance concepts to explain the discrepancy in the relationship between pressure gradient and fracture width, and to correlate pressure gradient, shear stress, flow rate, and shear rate.

\section{Rheology Measurements}

Materials. The polymer used for all experiments was a partially hydrolyzed polyacrylamide (HPAM): Ciba Alcoflood $935^{\mathrm{TM}}$ with a molecular weight of $5 \times 10^{6}$ daltons and $5-10 \%$ degree of hydrolysis. Chromium acetate was used as the crosslinker. Our standard 1X, 2X, and 3X gels were used in the measurements. The $1 \mathrm{X}$ gel contained $0.5 \%$ Alcoflood 935 HPAM, 0.0417\% $\mathrm{Cr}$ (III) acetate, $1 \% \mathrm{NaCl}$, and $0.1 \% \mathrm{CaCl}_{2}$. The multipliers of $\mathrm{X}$ refer to the HPAM and chromium acetate concentrations relative to those used in our standard $1 \mathrm{X}$ gel. In all cases, the $\mathrm{HPAM} / \mathrm{Cr}(\mathrm{III})$-acetate ratio was fixed at $12: 1$, and the gels were aged for 24 hours at $41^{\circ} \mathrm{C}$ before testing. 
Rheometer. Rheology measurements were performed with a Paar-Physica UDS $200^{\mathrm{TM}}$ Universal Dynamic Spectrometer fitted with a parallel-plate geometry MP 31 (50-mm diameter) for the wall-slip tests. The rheometer was equipped with a temperature control unit capable of maintaining the sample temperature at $41 \pm 0.2^{\circ} \mathrm{C}$. A parallel-plate geometry was used with double-rough surfaces to eliminate wall-slip effects. A cone-plate geometry MK 22 (25-mm diameter, $1^{0}$ ) was used for normal stress measurements. Samples were loaded using syringes without needles.

Procedures. First, steady shear measurements with controlled shear rate (CSR) were performed using different gap heights and various rough surfaces to identify the existence of wall-slip effects and to compare the results of wall-slip prevention measures. For the first set of tests, the gap between the parallel plates was set at $h_{1}=0.5 \mathrm{~mm}$. The upper plate was rotated at a fixed shear rate and the resultant stress measurement on the bottom plate was recorded. This test was repeated for a range of shear rates to obtain the flow curve (i.e., stress versus shear rate). For the next set of tests, the gap height was increased to $h_{2}=1 \mathrm{~mm}$, and the above processes were repeated to obtain another flow curve. These procedures were performed on smooth, sandblasted, profiled, and sandpaper-covered surfaces using our $1 \mathrm{X}, 2 \mathrm{X}$, and $3 \mathrm{X}$ gels.

Second, amplitude and frequency sweeps were performed to verify the existence of wall-slip effects and to determine the yielding area. Strain amplitude sweeps were performed at a constant frequency of $1 \mathrm{~Hz}$ over the strain range of $1-10^{5} \%$ (i.e., factor of 0.1 to 1000 total change) to obtain storage modulus $\left(G^{\prime}\right)$ and loss modulus $\left(G^{\prime \prime}\right)$. Frequency sweeps were conducted at $10 \%$ strain to ensure linear viscoelastic response over the frequency range from $0.1-10 \mathrm{~Hz}$. Frequency sweep was used to compare dynamic moduli with values obtained from the amplitude sweep. These measurements were conducted on smooth, sandblasted, profiled, and sandpaper-covered surfaces with our $1 \mathrm{X}, 2 \mathrm{X}$, and $3 \mathrm{X}$ gels.

Steady shear tests with controlled shear stress (CSS), also known as creep tests, were performed to determine yield stress. Stress was incremented stepwise from 10 to $500 \mathrm{~Pa}$, depending on the different gels used, and the resultant shear rate was recorded. When shear stress was applied above the yield stress, the gels typically reached steady state in less than 10 seconds. Yield stress values were defined as the lowest points at which stress produced steady state deformation. The time required for each step ranged from about 10 to 100 seconds.

Steady shear tests with controlled shear rate were also performed to measure first normal stress difference. Normal force $\left(F_{N}\right)$ was preset at zero prior to loading the sample; the sample was then loaded and a wait period ensued until the normal force returned to the original preset value, or a stable value close to zero. The shear rate was preset to increase stepwise from 0.005 to $1000 \mathrm{~s}^{-1}$, depending on the material, and the rheometer recorded the resultant first normal stress difference. The time to reach steady state ranged from about 0.1 to $100 \mathrm{~s}$, depending on the shear rate: the smaller the shear rate, the longer the time required to reach steady state. Measurements of first normal stress difference were performed using our $1 \mathrm{X}, 2 \mathrm{X}$, and $3 \mathrm{X}$ gels.

\section{Wall Slip}

Literature Review. When gel is extruded through a fracture, a minimum pressure gradient must be applied in order for the gel to flow, which suggests the gel material exhibits a yield 
stress. $^{23,72,73,85}$ The yield point is the end of a reversible elastic deformation and the beginning of an irreversible deformation, or viscoelastic/viscous flow. In the low deformation range, the material exhibits a stable, solid-like structure which displays elastic behavior. But when the deformation exceeds the tolerance of the 3-D network, the structure is destroyed and the material flows. For a gel with yield stress, $\tau_{y}$, under stationary (steady rate) conditions, Bird et al. ${ }^{84}$ used a simple force balance to calculate the minimum pressure gradient, $d p / d l$, required for gel to extrude through two parallel plates separated by a distance of $w$ :

$$
d p / d l=2 \tau y / w
$$

Employing a UDS 200 rheometer with a smooth surface, parallel-plate geometry, Liu ${ }^{82}$ estimated the yield stress of a $1 \mathrm{X} \mathrm{Cr}$ (III)-acetate-HPAM gel in the range of 10 to $88 \mathrm{~Pa}$. Using these values and an aperture of $1 \mathrm{~mm}(0.04 \mathrm{in})$, Eq. 8 predicted pressure gradients between 20 and $176 \mathrm{kPa} / \mathrm{m}$ $(0.9$ and $7.8 \mathrm{psi} / \mathrm{ft})$. In contrast, the experimentally measured values ranged from 633 to 1,130 $\mathrm{kPa} / \mathrm{m}$ (28 to $50 \mathrm{psi} / \mathrm{ft}$ ) when the fracture width was $1 \mathrm{~mm} .^{7}$ Therefore, the yield stresses from Liu greatly underestimated the pressure gradients required for gel extrusion through fractures.

What factors might lower the measured value of yield stress? Macosko ${ }^{86}$ mentioned that for viscoelastic materials, wall-slip effects were particularly prevalent during yield stress measurements. He noted that because of the impenetrability of the wall, the layer of particles adjacent to the rheometer wall was typically more dilute than the bulk dispersion. During flow, the shear rate gradient caused particles to migrate away from the wall. The thin, dilute layer near the wall had a much lower viscosity - creating the impression that the bulk fluid was slipping along the wall. Since the yield point was a flow/no-flow point as well as a transition point from elastic solid to viscous liquid, the existing thin, dilute layer acted as if the yield point had been reached and the material had already started to flow. The measured yield stress, in this case, was lower than its actual value. When measuring rheology properties using a smooth-surface geometry, wall-slip effects could exist and diminish the measured yield stress. ${ }^{81-84}$ Therefore, wall-slip effects must be identified and eliminated to ensure that the measured results are properties of the gels and not artifacts produced by the method of investigation.

In our research, we used the method outlined by Yoshimura and Prud'homme ${ }^{87,88}$ to analyze wall-slip effects. Two assumptions were made when using this method: 1) that wall layer thickness was small compared to the viscometer gap, and 2) that slip velocity was only a function of stress once steady state was achieved. Yoshimura and Prud'homme measured stress versus shear rate with two different gap heights, $h_{1}$ and $h_{2}$. They found that if wall slip did not exist, the sample was purely sheared and the rheometer gap size would not play any role in the measurements. On the other hand, if wall slip occurred, the apparent viscosity increased with greater gap height. As gap height increased, the thin lubricating layer that was responsible for slip became less important.

According to Yoshimura and Prud'homme, the actual (corrected) shear rate at a given stress, $\tau_{R \text {, }}$ is

$$
\dot{\gamma}_{R}\left(\tau_{R}\right)=\frac{h_{1} * \dot{\gamma}_{a R 1}\left(\tau_{R}\right)-h_{2} * \dot{\gamma}_{a R 2}\left(\tau_{R}\right)}{h_{1}-h_{2}}
$$


with slip velocity

$$
u_{s}\left(\tau_{R}\right)=\frac{\dot{\gamma}_{a R 1}\left(\tau_{R}\right)-\dot{\gamma}_{a R 2}\left(\tau_{R}\right)}{2 *\left(\frac{1}{h_{1}}-\frac{1}{h_{2}}\right)}
$$

and with corrected viscosity

$$
\eta\left(\dot{\gamma}_{R}\right)=\frac{\left(h_{1}-h_{2}\right){ }^{*} \tau_{R}}{h_{1} \dot{\gamma}_{a R 1}\left(\tau_{R}\right)-h_{2} \dot{\gamma}_{a R 2}\left(\tau_{R}\right)},
$$

where $\dot{\gamma}_{a R 1}\left(\tau_{R}\right), \dot{\gamma}_{a R 2}\left(\tau_{R}\right)$ are the apparent shear rates at a given shear stress.

Steady Shear Tests (CSR) for Flow Curve. Flow curves for 1X, 2X, and 3X gels using the smooth-surface rheometer at 0.5- and 1-mm gap heights were plotted (Fig. 21). All three gels were shear-thinning materials. The flow curves plotted for $1 \mathrm{X}$ and $2 \mathrm{X}$ gels clearly show higher shear stresses at the 1-mm gap height (solid squares) than those at $0.5 \mathrm{~mm}$ (open diamonds). The separation in the flow curves for these two gap heights suggests a wall-slip effect on the measurements. However, flow curves for the $3 X$ gel with gap heights of $0.5 \mathrm{~mm}$ and $1 \mathrm{~mm}$ overlapped, indicating that the wall-slip effect was negligible.

The above measurements were repeated using rough surfaces to eliminate wall-slip effects. Sandpaper was used to cover both the bottom and upper plates. Flow curves for $1 \mathrm{X}$ and $2 \mathrm{X}$ gels are shown in Fig. 22 for the double-rough surfaces at two different gap heights. For the 1X and $2 \mathrm{X}$ gels, the flow curves were fairly close; thus, the wall-slip effects were significantly reduced by the double-rough surface. For the $3 \mathrm{X}$ gel, flow curves for the various surfaces were not significantly different.

Using Yoshimura and Prud'homme's method to correct for wall slip on the smooth surface, we generated corrected flow curves for the $1 \mathrm{X}$ and $2 \mathrm{X}$ gels and compared these with those for double-rough surfaces. The corrected flow curves for smooth surfaces are shown by the solid curves in Fig. 22. The corrected flow curves generally overestimated the shear stress at low shear rates and underestimated the shear stress values at high shear rates. More work is needed to understand this behavior.

Oscillatory Measurements. Oscillatory measurements were also performed to detect wall-slip effects and to verify the viscoelastic property of the gels. Gap heights were varied from 0.5 to 2 $\mathrm{mm}$ and various surfaces were employed for evaluating the presence of wall-slip effects. Fig. 23 compares storage $\left(G^{\prime}\right)$ and loss $\left(G^{\prime \prime}\right)$ moduli for a $1 \mathrm{X}$ gel on smooth and rough surfaces at various gap heights. 

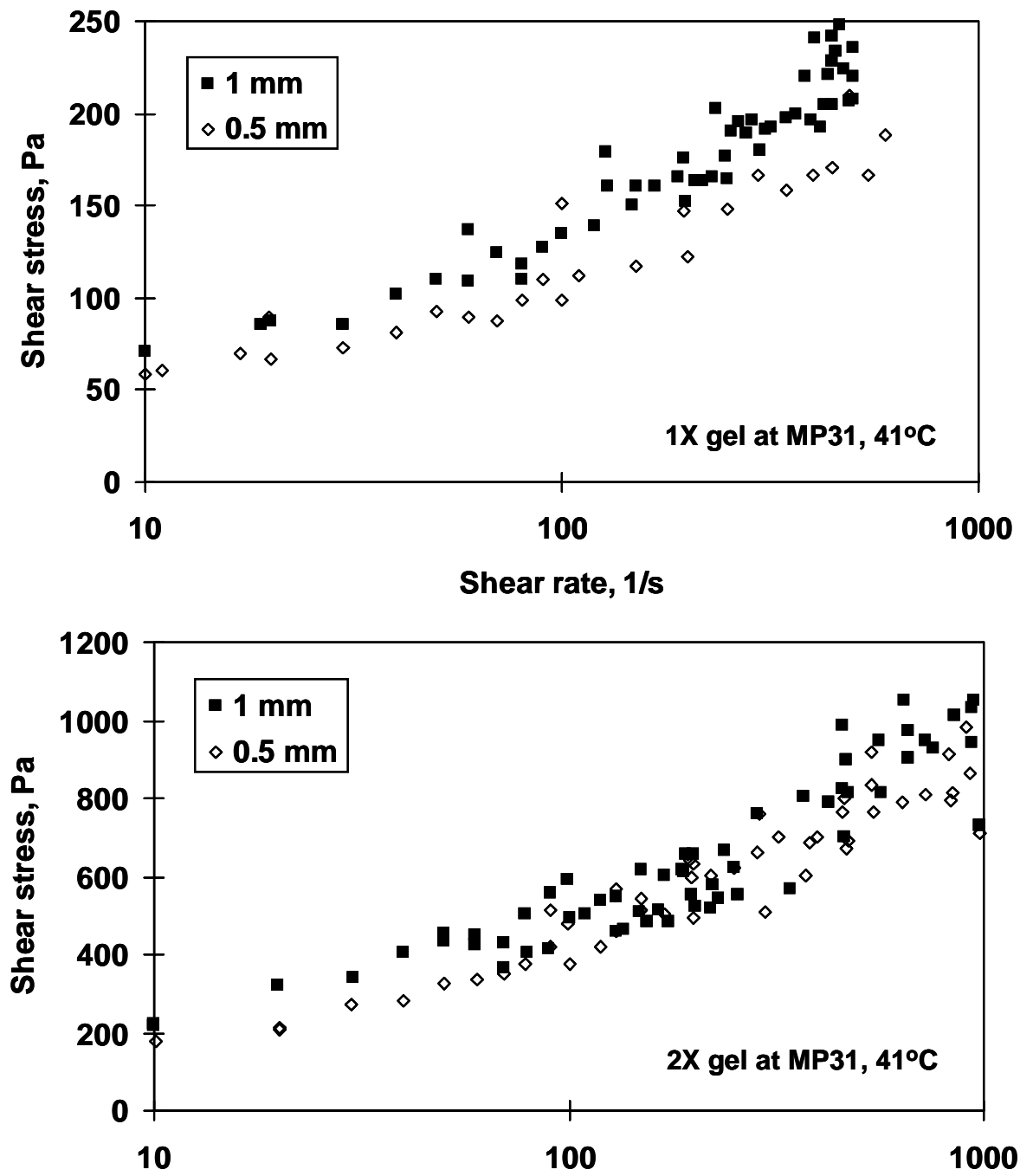

Shear rate, $1 / \mathrm{s}$

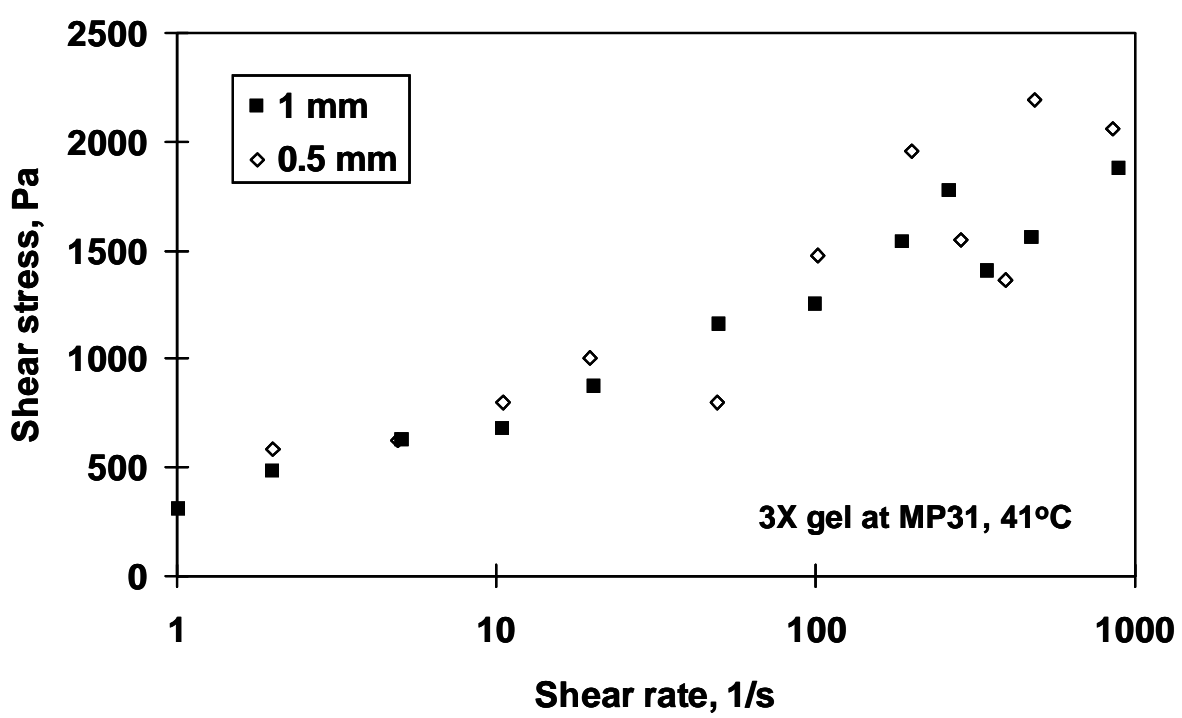

Fig. 21-Flow curves for $1 \mathrm{X}, 2 \mathrm{X}$, and $3 \mathrm{X}$ gels on smooth surfaces. 

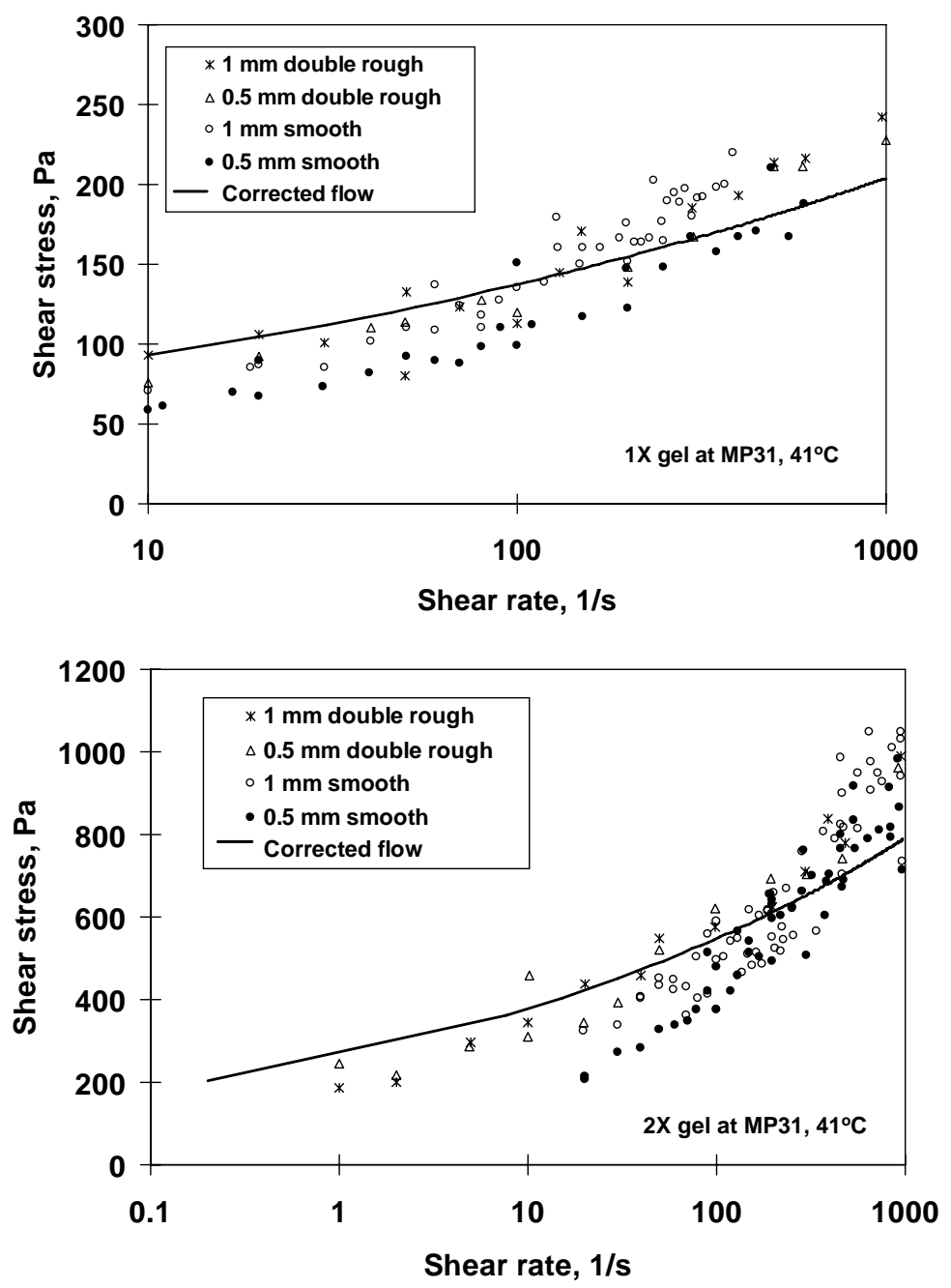

Fig. 22-Flow curve comparison of $1 \mathrm{X}$ and $2 \mathrm{X}$ gels on different surfaces.

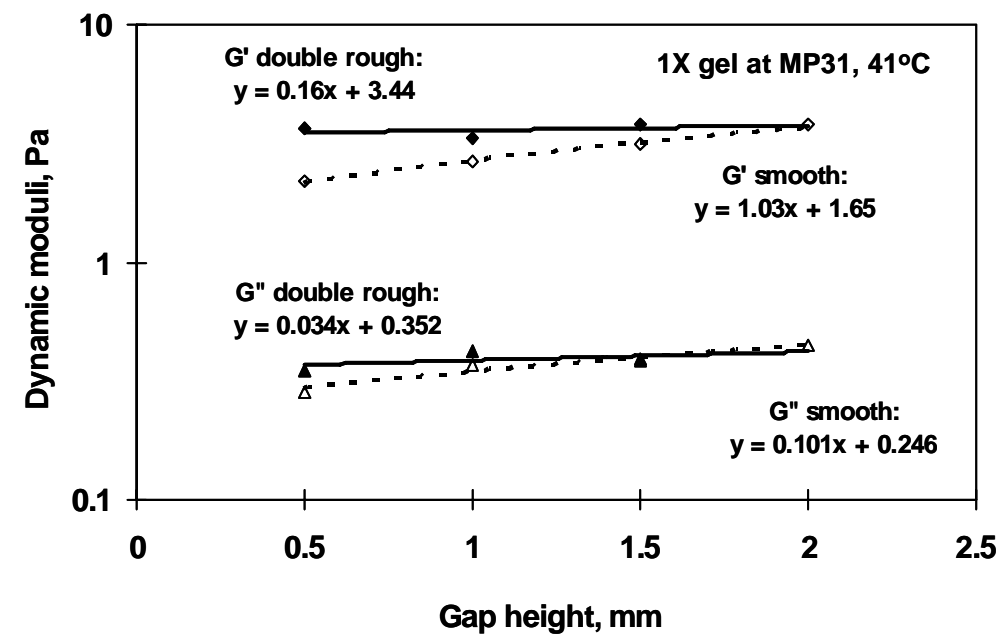

Fig. 23-Dynamic property comparison for a $1 \mathrm{X}$ gel versus gap on different surfaces. 
This figure reveals the following points for the $1 \mathrm{X}$ gel: (a) on smooth surfaces, both storage and loss moduli increased with gap height, indicating the presence of wall-slip effects; (b) on doublerough surfaces, storage and loss moduli were nearly independent of gap height, indicating that the wall-slip effects were reduced by the rough surfaces; and (c) storage and loss moduli on double-rough surfaces were larger than those measured on smooth surfaces, indicating that wallslip effects were reduced by double-rough surfaces. These conclusions were consistent with observations from the steady shear tests. Similar studies using the $2 \mathrm{X}$ and $3 \mathrm{X}$ gels were less definitive.

Creep Tests. Creep tests were performed using the double-rough surfaces to determine yield stress (i.e., when the gels began to flow). Fig. 24 shows creep test results for our $1 \mathrm{X}, 2 \mathrm{X}$, and $3 \mathrm{X}$ gels. The yield stress was identified when the strain-versus-time curves became sensitive to the applied stress. For example, for the 1X gel, the strain-versus-time curves were insensitive to the applied stress below $71 \mathrm{~Pa}$; above $71 \mathrm{~Pa}$ the strain-versus-time curves were significantly higher. From Fig. 24, the yield stress was $72 \mathrm{~Pa}$ for the $1 \mathrm{X}$ gel, $141 \mathrm{~Pa}$ for the $2 \mathrm{X}$ gel, and about $400 \mathrm{~Pa}$ for the $3 \mathrm{X}$ gel. Liu ${ }^{82,83}$ measured the yield stress of a $1 \mathrm{X}$ gel by tangent crossover on the flow curve. She listed three possible values for yield stress, from 10 to $88 \mathrm{~Pa}$. She had difficulty determining the actual yield point. The yield stress from our measurements on the $1 \mathrm{X}$ gel was closer to her highest value. The other two possible choices for yield point from Liu's work may have been influenced by wall slip.

Table 6 summarizes the above measurements and analysis of rheology properties for our 1X, 2X, and $3 \mathrm{X}$ gels.

Table 6-Rheology properties of Cr(III)-acetate-HPAM gels at $41^{\circ} \mathrm{C}$

\begin{tabular}{|c|c|c|c|c|c|}
\hline Material & $\begin{array}{c}\text { Yield } \\
\text { Stress } \\
\tau_{y}, \mathrm{~Pa}\end{array}$ & $\begin{array}{c}\text { Consistency } \\
\text { Index } \\
k\end{array}$ & $\begin{array}{c}\text { Power } \\
\text { Index } \\
n\end{array}$ & $\begin{array}{c}\text { Storage } \\
\text { Modulus } \\
G^{\prime}, \mathrm{Pa}\end{array}$ & $\begin{array}{c}\text { Loss } \\
\text { Modulus } \\
G^{\prime \prime}, \mathrm{Pa}\end{array}$ \\
\hline 1X gel & 72 & 41.5 & -0.75 & 3.81 & 0.444 \\
\hline 2X gel & 141 & 219 & -0.79 & 36.1 & 0.587 \\
\hline 3X gel & 400 & 537 & -0.81 & 117 & 1.90 \\
\hline
\end{tabular}

The consistency index and power index were obtained by fitting flow curves to the stress-versusshear-rate values from tests with controlled shear rate (Fig. 21). The flow behavior of 1X, 2X, and $3 \mathrm{X}$ gels were described by the Herschel-Bulkley model ${ }^{86}$ as follows:

For 1X gel: $\tau=72+41.5 \dot{\gamma}^{0.25}(\tau>72 \mathrm{~Pa})$

For 2X gel: $\tau=141+219 \dot{\gamma}^{0.21}(\tau>141 \mathrm{~Pa})$

For 3X gel: $\tau=400+537 \dot{\gamma}^{0.19}(\tau>400 \mathrm{~Pa})$

When the shear stresses were lower than the yield stress, shear rates were zero. 
Using the yield stress, we calculated the pressure gradient required for gel extrusion through the fracture using the simple force balance approach. Assuming 1X gel was injected into a 1-mm wide fracture, the pressure gradient was $143 \mathrm{kPa} / \mathrm{m}(6.3 \mathrm{psi} / \mathrm{ft})$. This prediction was comparable to that of Liu-still much less than the results from the extrusion experiments. The main value of this investigation was that we identified a more reliable means to determine the yield stresses for our 1X, 2X, and $3 \mathrm{X}$ gels. We also established that wall slip was not primarily responsible for the apparent discrepancy between rheology measurements and extrusion results in fractures.
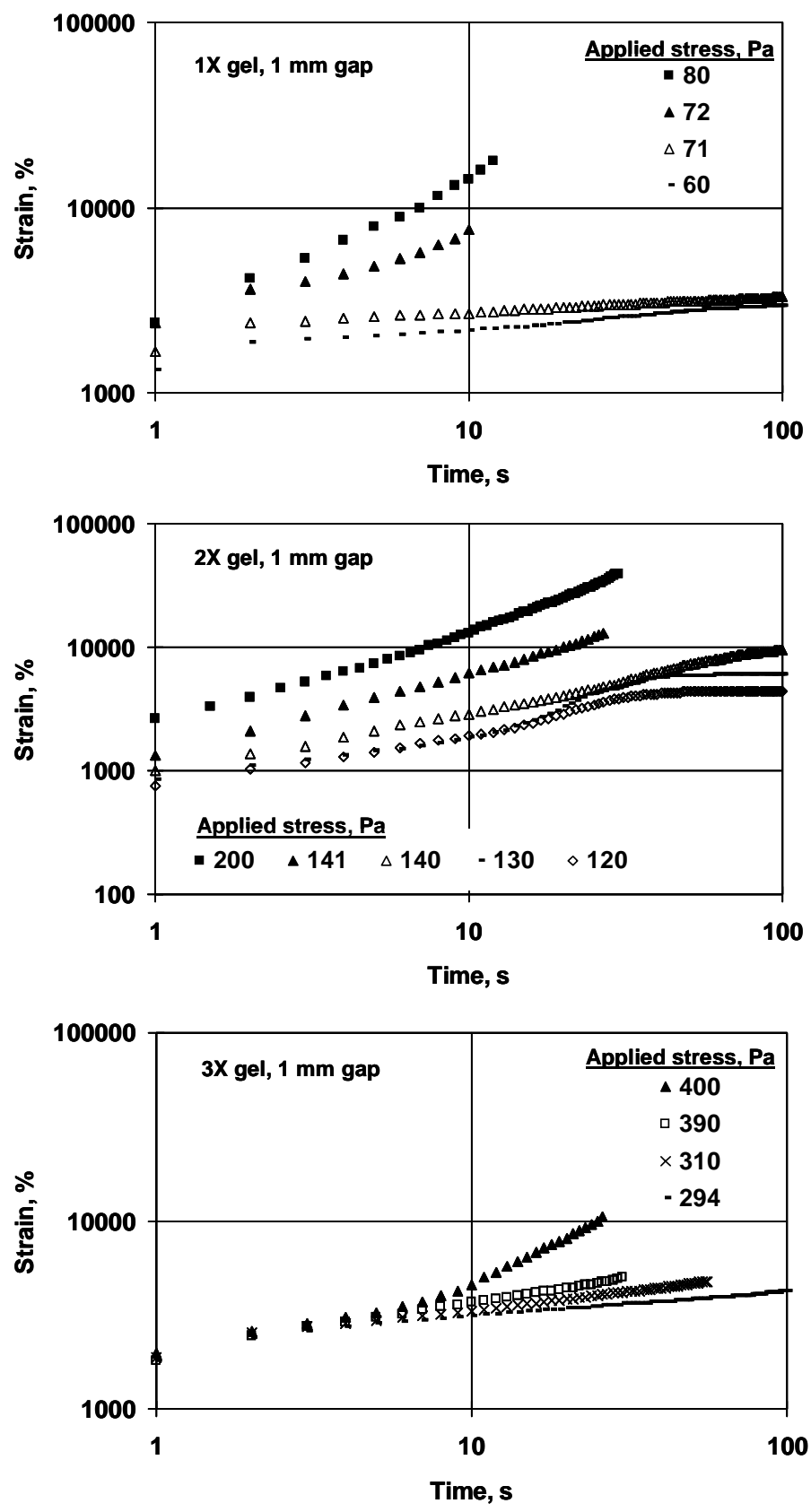

Fig. 24-Creep tests for $1 \mathrm{X}, 2 \mathrm{X}$, and $3 \mathrm{X}$ gels on MP31, double-rough surface at $41^{\circ} \mathrm{C}$. 


\section{First Normal Stress Difference}

Literature Review. We wondered whether normal stress differences were responsible for the unexpectedly high pressure gradients during gel extrusion through fractures. Previous researchers studied first normal stress difference in the form of extrudate, or die swell ${ }^{95-97}$ (i.e., the expansion that occurs when a viscoelastic liquid leaves a capillary die), or as the Weissenberg effect ${ }^{98,99}$ (i.e., during stirring, viscoelastic liquids creep up the stir shaft—also known as stir-rod climbing). In these previous studies, first normal stress difference was measured in steady shear flows ${ }^{95,100-104}$ or was predicted by models. ${ }^{95-97,105}$ For polymers, the steady shear response for the first normal stress difference, $N_{l}$, usually exhibits the maximum slope at low shear rates and then gradually flattens out as shear rate increases. ${ }^{102}$

Both the die swell and Weissenberg effects are related to first normal stress difference. With a simple viscous fluid, only the resistance force $(F)$ or the shear stress $(\tau)$ occurs and acts in the direction of flow, but no force acts perpendicular to the wall. With a viscoelastic material, a normal force $\left(F_{N}\right)$ can occur that presses the two shearing areas apart. The normal stress $(T)$ occurs due to anisotropic microstructures present in the viscoelastic material. When a viscoelastic material is sheared between two parallel surfaces (Fig. 25), two normal stress differences are produced in addition to the viscous shear stress; these two normal stress differences are $N_{1}=T_{11}-T_{22}$ and $N_{2}=T_{22}-T_{33}$. The subscripts are defined as follows: "1" is the flow direction, "2" is the direction perpendicular to the surfaces between which the fluid is sheared, and " 3 " is the neutral direction.

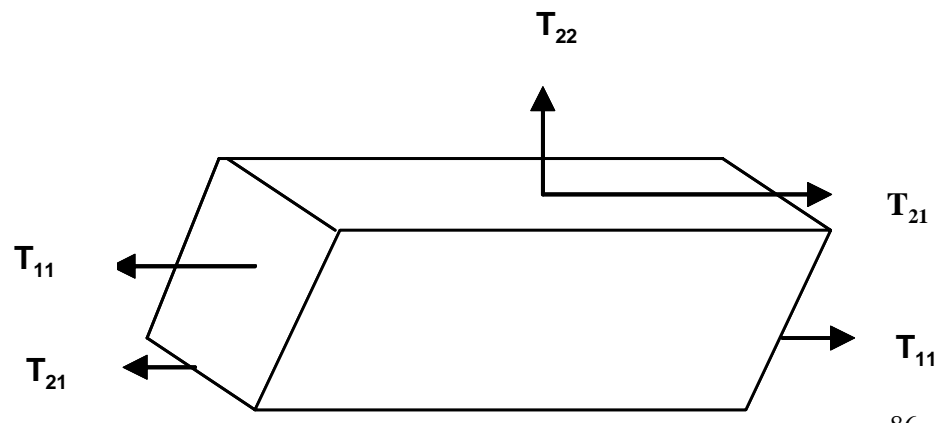

Fig. 25-Viscous shear stress and normal stresses. ${ }^{86}$

The larger of the two normal stress differences is $N_{l}$, and it is responsible for the rod-climbing phenomenon. For isotropic materials, $N_{l}$ is positive in sign (unless it is zero). In a rheometer with cone-plate geometry, $N_{l}$ causes the cone and plate surfaces to be pushed apart.

Results and Discussion. During our extrusion experiments in 1-mm wide fractures, the injection rates were between 0.046 and $23.4 \mathrm{~cm} / \mathrm{s}$, which translate to shear rates (at the wall) between 0.92 and $468 \mathrm{~s}^{-1}$. We performed steady shear measurements in our rheometer to determine the first normal stress difference within this range of shear rates.

As expected for our $1 \mathrm{X}, 2 \mathrm{X}$, and $3 \mathrm{X}$ gels, the first normal stress difference increased with shear rate for all three gels (Fig. 26). $N_{l}$ was larger for the more concentrated gels, which is reasonable since more concentrated gels have greater elasticity. 
For the 1X gel, the first normal stress difference increased from 442 to $19,000 \mathrm{~Pa}$ when the shear rate increased from 0.2 to $1,000 \mathrm{~s}^{-1}$. At shear rates below $100 \mathrm{~s}^{-1}, N_{l}$ was proportional to the square root of shear rate, $\dot{\gamma}^{0.5}$. For shear rates between 100 and $1,000 \mathrm{~s}^{-1}, N_{l}$ was proportional to $\dot{\gamma}^{0.25}$.

For the $2 \mathrm{X}$ gel, the first normal stress difference increased from 1,000 to $19,900 \mathrm{~Pa}$ when the shear rate increased from 0.05 to $100 \mathrm{~s}^{-1}$. When the shear rate was below $0.2 \mathrm{~s}^{-1}$, the slope of the $N_{1}$ curve was about 2. At higher shear rates, the first normal stress difference was fairly insensitive to shear rate.

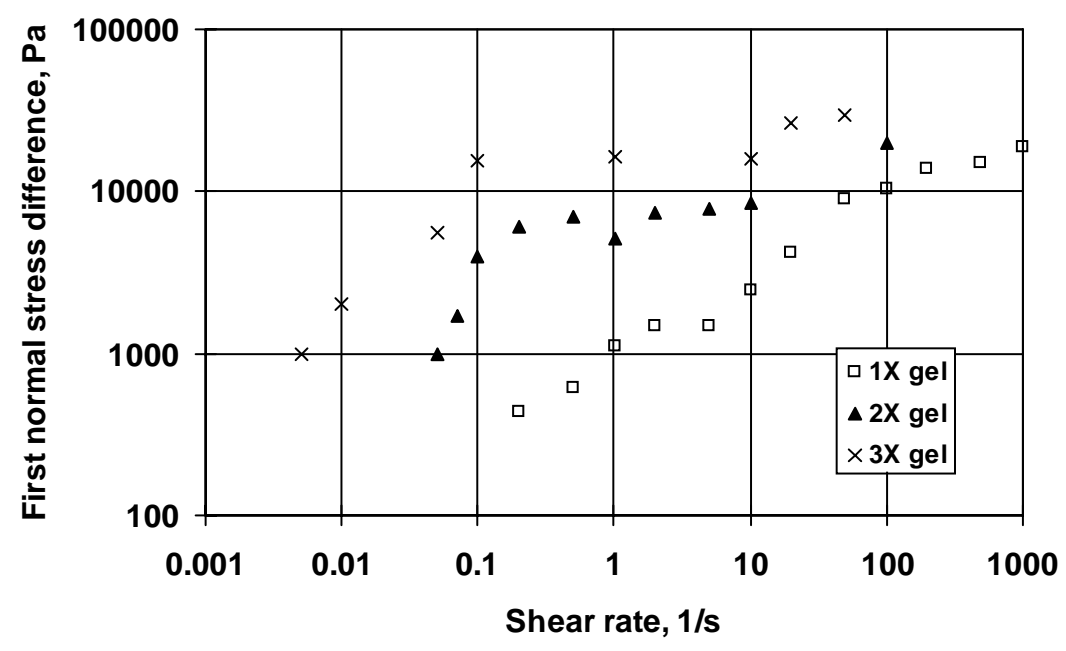

Fig. 26-First normal stress difference versus shear rate for $1 \mathrm{X}, 2 \mathrm{X}$, and $3 \mathrm{X}$ gels at $41^{\circ} \mathrm{C}$.

For the $3 \mathrm{X}$ gel, the first normal stress was between 1,000 and 43,200 $\mathrm{Pa}$ in the shear rate range from 0.005 to $60 \mathrm{~s}^{-1}$. For shear rates below $0.1 \mathrm{~s}^{-1}$, the slope of the $N_{l}$ curve was about 1 . For shear rates between 0.1 and $100 \mathrm{~s}^{-1}$, the first normal stress difference was fairly insensitive to shear rate. This behavior is qualitatively consistent with polymer behavior that was reported earlier. $^{102}$

The first normal stress difference depends on shear rate and flow geometry. ${ }^{101}$ Therefore, for steady state flow through a fracture with smooth faces, the first normal stress difference should remain constant along the fracture. However, since fracture faces typically have rough surfaces, fracture width typically varies along the fracture length. Thus, even at the same flow rate, the shear rate could be different in different portions of the fracture. As a result, the first normal stress difference could vary along the fracture and varying pressures might be exerted on the fracture faces. Can this observation explain the difference in pressure gradient found between gel extrusion experiments in fractures and expectations from rheology measurements?

Assuming that the fracture width was $1 \mathrm{~mm}$ when a $1 \mathrm{X}$ gel was injected, the predicted pressure gradient was $143 \mathrm{kPa} / \mathrm{m}(6.3 \mathrm{psi} / \mathrm{ft})$ from the simple force balance approach, compared to 633 to $1,130 \mathrm{kPa} / \mathrm{m}(28$ to $50 \mathrm{psi} / \mathrm{ft})$ in the extrusion experiments. ${ }^{23,73}$ Fig. 26 shows that the first normal stress for the 1X gel was between 442 and 19,000 Pa in the shear rate range from 0.2 to $1,000 \mathrm{~s}^{-1}$. 
If a constriction reduced the effective fracture width by $50 \%$, the shear rate should increase by a factor of 8 (i.e., $2^{3}$ ). From Fig. 26, this variation in shear rate should not change the normal stress by more than a factor of four for the $1 \mathrm{X}$ gel. If normal stress is reduced from $10,000 \mathrm{~Pa}$ to 2,500 $\mathrm{Pa}$ when passing through a constriction, a pressure change of 7,500 $\mathrm{Pa}$ (1.1 psi) might be experienced. If 20 such constrictions were encountered along the fracture, this behavior could explain the higher pressure gradients observed during gel extrusion through fractures. However, during our extrusion experiments, we generally used fractures that did not have pronounced constrictions. More importantly, we often used fractures with smooth faces, and the pressure gradients during extrusion of a $1 \mathrm{X}$ gel through a 1-mm wide fracture were still from 633 to 1,130 $\mathrm{kPa} / \mathrm{m}$ (28 to $50 \mathrm{psi} / \mathrm{ft}){ }^{23,73}$ Thus, although normal forces could add significantly to the pressure gradients observed when using fractures with many pronounced constrictions, they do not explain the high pressure gradients observed during the bulk of our gel extrusion experiments.

\section{Gel Dehydration and Wormholes in Fractures}

Before gelation, fluid gelant solutions can readily leak off from fractures into porous rock. However, after gelation, the crosslinked materials will not penetrate significantly into the porous rock. $^{23,72,73}$ Thus, formed gels must extrude through fractures during the placement process. $\mathrm{Cr}$ (III)-acetate-HPAM gels (as well as other gels) concentrate or lose water during extrusion through fractures. As water leaks off from the gel, the gel concentrates (by factors up to 50) to become immobile in the vicinity where dehydration occurs. The driving force for gel dehydration (and water leakoff) is the pressure difference between the fracture and the adjacent porous rock. Because fresh gel (i.e., mobile gel, with the original composition) is much more pliable and mobile than the concentrated gel, the fresh gel wormholes through the concentrated gel in order to advance the gel front. ${ }^{23,72,73-7}$ These wormholes may be significantly narrower than the fracture width. This possibility could explain the relatively high pressure gradients during gel extrusion.

By inputting a pressure gradient of $633 \mathrm{kPa} / \mathrm{m}(28 \mathrm{psi} / \mathrm{ft})$ and a yield stress of $35 \mathrm{~Pa}(0.005 \mathrm{psi})$ (from $\mathrm{Liu}^{82,83}$ ) into a simple force balance, Seright ${ }^{23}$ estimated the actual opening width to be about $0.1 \mathrm{~mm}$ - one-tenth of the fracture width. Similarly, using a pressure gradient range of 633 to $1,130 \mathrm{kPa} / \mathrm{m}$ (28 to $50 \mathrm{psi} / \mathrm{ft})$ with our measured yield stress value of $72 \mathrm{~Pa}(0.01 \mathrm{psi})$, the calculated apertures were from 0.13 to $0.23 \mathrm{~mm}$. Thus, the relatively high pressure gradients during gel extrusion through fractures may have resulted because the actual gel flow path (i.e., through wormholes) was significantly narrower than the fracture width.

\section{Pressure Gradient versus Fracture Width}

Literature Review. A force balance ${ }^{84}$ suggested that the minimum pressure gradients required for gel extrusion through fractures should be inversely proportional to fracture width or capillary radius. In a capillary of radius, $R$,

$$
d p / d l=2 \tau_{y} / R \text {. }
$$

Ganguly et $a l .{ }^{89}$ demonstrated that rupture pressure was inversely proportional to the inside diameter of the tube when gels $(0.75 \%$ Alcoflood 935, $0.0417 \% \mathrm{Cr}$ (III) acetate, $1 \% \mathrm{NaCl}$; gel time $=12$ hours, gelant aged 6 to 8 days) were placed in various lengths of nylon and polypropylene tubes with different inside diameters. Seright ${ }^{85}$ noted similar results when studying failure of the $1 \mathrm{X}$ gel in tubes made of stainless steel, Teflon, glass, and 
polyetheretherketone. A power regression on the data confirmed that the failure pressure gradient was inversely proportional to the tube diameter. However, the correlation coefficient for the regression was only 0.6 .

Seright $^{23,72,73}$ also noted that the pressure gradient required for gel extrusion was inversely proportional to the square of the fracture width (Fig. 27). For our 1X $\mathrm{Cr}$ (III)-acetate-HPAM gel, the required pressure gradient $(d p / d l$, in psi/ft) could be estimated using Eq. 9 (if fracture width, $w_{f}$, is expressed in inches):

$$
d p / d l=0.02 /\left(w_{f}\right)^{2}
$$

We wondered why the pressure gradient required for gel extrusion through an open channel was inversely proportional to the channel width for the simple force balance approach, but was inversely proportional to the square of the fracture width in the extrusion experiments.

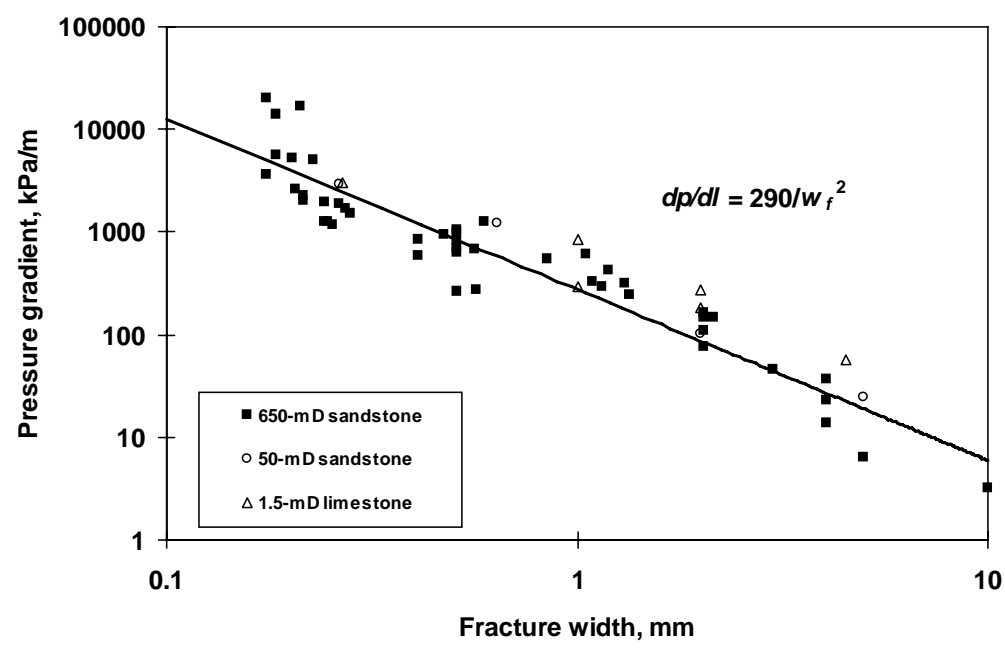

Fig. 27-Pressure gradients required for gel extrusion. ${ }^{73}$

To describe gel behavior in the fracture, Seright ${ }^{72}$ examined a Bingham model for extrusion of the $\mathrm{Cr}$ (III)-acetate-HPAM gels through fractures. In this model, a Newtonian fluid near the fracture wall lubricated the flow of the solid-like materials in the middle of the path. Water leaving the gel during the dehydration process could comprise the lubricating layer. The thickness of the lubricating layer should be small relative to the fracture width and increase linearly with fluid velocity.

Two flaws existed with the Bingham model. First, the pressure gradients predicted by this model should depend on the viscosity of the lubricating fluid-water in this case. Since the viscosity of water decreased by a factor of about three as temperature increased from $20^{\circ}$ to $80^{\circ} \mathrm{C}$, the measured pressure gradients for gel extrusion should also have decreased by roughly a factor of three. In reality, the pressure gradient was insensitive to temperature over this range. Second, the lubrication layer should have become thicker with increased flow rate. With a thicker lubrication layer (i.e., more water), the leakoff rate should have increased with the increased extrusion rate. Instead, the leakoff rate was independent of flow rate-it was primarily time-dependent. ${ }^{72,73}$ 
In view of the above deficiencies, we considered an alternative model that was based on the Herschel-Bulkley model, ${ }^{86}$ where the fluid exhibited a yield stress and flowed as a power-law fluid above the yield point. We employed a shell momentum balance ${ }^{91}$ and power-law equations to deduce these models.

Model Based on Shell Momentum Balance and Power-Law. For steady-state flow, a momentum balance for a shell of finite thickness was first applied. As the thickness approached zero, the corresponding differential equation describing the momentum flux distribution was obtained. Next, the appropriate Newtonian or non-Newtonian expression for the momentum flux was inserted to obtain a differential equation for the velocity distribution. Integration of these two differential equations yielded the momentum flux and the velocity distributions for the system. This information can then be used to calculate various other quantities, such as average velocity, maximum velocity, volumetric flow rate, pressure drop, and forces on boundaries.

For fluid flow inside a tube, ${ }^{91}$

$$
\tau=\frac{p_{0}-p_{L}}{2 L} R
$$

where $R$ is the radius and $L$ is the length of the tube, and $p_{0}$ and $p_{L}$ are the inlet and outlet pressures, respectively. For Newtonian materials,

$$
\tau=-\eta \frac{d \gamma}{d t}
$$

where $\tau$ is the shear stress, $\eta$ is the Newtonian viscosity, $\gamma$ is shear strain, and $t$ is time.

Combining Eqs. 17 and 18, the velocity distribution along the radial direction is

$$
v=-\frac{p_{0}-p_{L}}{4 \eta L} R^{2}\left[\left(\frac{r}{R}\right)^{2}-1\right]
$$

where $v$ is the velocity and $r$ is the distance from the tube center.

Integrating Eq. 19, the volumetric flow rate is

$$
q=\frac{\pi\left(p_{0}-p_{L}\right) R^{4}}{8 \eta L}
$$

The pressure gradient versus tube radius is

$$
\frac{d p}{d l}=8 \eta \frac{q}{\pi} \frac{1}{R^{4}}
$$

Thus, for Newtonian materials, the pressure gradient varied inversely with the fourth power of 
tube radius.

For non-Newtonian materials, the power-law is

$$
\tau=-k\left(\frac{d v}{d t}\right)^{n+1}
$$

where $k$ is the consistency index and $n$ is the power index.

The velocity distribution along the tube radius is

$$
v=\left(-\frac{p_{0}-p_{L}}{2 k L}\right)^{\frac{1}{n+1}} \frac{n+1}{n+2}\left(r^{\frac{n+2}{n+1}}-R^{\frac{n+2}{n+1}}\right),
$$

and the volumetric flow rate is

$$
q=\left(-\frac{p_{0}-p_{L}}{2 k L}\right)^{\frac{1}{n+1}} \pi\left(-\frac{n+1}{3 n+4}\right) R^{\frac{3 n+4}{n+1}}
$$

The pressure gradient versus tube radius is

$$
\frac{d p}{d l}=(-2 k)\left(-\frac{3 n+4}{n+1}\right)^{n+1}\left(\frac{q}{\pi}\right)^{n+1} \frac{1}{R^{3 n+4}}
$$

Therefore, the pressure gradient varied inversely with the tube radius raised to the power, $3 n+4$, for power-law, non-Newtonian materials.

For fluids flowing between two parallel plates, the shell momentum balance reveals that

$$
\tau=\left(\frac{p_{0}-p_{L}}{L}\right) x
$$

where $x$ is the distance from the center of the fracture to the fracture wall.

Following the same procedures used for tubes, for Newtonian materials, the velocity distribution along the fracture width $(w)$ is

$$
v_{z}=-\frac{p_{0}-p_{L}}{2 \eta L} w^{2}\left[\left(\frac{x}{w}\right)^{2}-\frac{1}{4}\right]
$$

The volumetric flow rate is

$$
q=\frac{1}{12} \frac{\left(p_{0}-p_{L}\right)}{\eta L} h w^{3},
$$


where $h$ is the fracture height and $L$ is the fracture length.

The pressure gradient versus fracture width is

$$
\frac{d p}{d l}=\frac{12 \eta q}{h} \frac{1}{w^{3}}
$$

Therefore, the pressure gradient varied inversely with the third power of fracture width for Newtonian materials.

For non-Newtonian materials following the power-law, the velocity distribution along the fracture width is

$$
v=\left(-\frac{p_{0}-p_{L}}{k L}\right)^{\frac{1}{n+1}} \frac{n+1}{n+2}\left(\frac{w}{2}\right)^{\frac{n+2}{n+1}}\left[\left(\frac{2 x}{w}\right)^{\frac{n+2}{n+1}}-1\right]
$$

and the volumetric flow rate is

$$
q=\left(-\frac{p_{0}-p_{L}}{2 k L}\right)^{\frac{1}{n+1}} \frac{h}{2}\left(-\frac{n+1}{2 n+3}\right) w^{\frac{2 n+3}{n+1}}
$$

The pressure gradient versus fracture width is

$$
\frac{d p}{d l}=(-2 k)\left(-\frac{2 n+3}{n+1}\right)^{n+1}\left(\frac{2 q}{h}\right)^{n+1} \frac{1}{w^{2 n+3}}
$$

Therefore, for power-law, non-Newtonian materials, the pressure gradient varied inversely with the fracture width, raised to the power, $2 n+3$.

Summarizing the above equations, the pressure gradients required for Newtonian materials to extrude varied inversely with the fourth power of tube radius and with the third power of fracture width. For power-law, non-Newtonian materials, the pressure gradients varied inversely with tube radius raised to the power, $3 n+4$, and with fracture width raised to the power, $2 n+3$.

The relationship of pressure gradient versus tube radius/fracture width for our $1 \mathrm{X}, 2 \mathrm{X}$, and $3 \mathrm{X}$ gels is summarized in Table 7. For the gels listed in this table, $q$ must have units $\mathrm{of} \mathrm{m}^{3} / \mathrm{s} ; R, w$, and $h$ must have units of $\mathrm{m} ; \eta$ must have units of Pa-s; and the calculated pressure gradients, $d p / d l$, must have units of $\mathrm{Pa} / \mathrm{m}$. 
Table 7-Pressure gradient versus tube radius/fracture width for Cr(III)-acetate-HPAM gels

\begin{tabular}{|c|c|c|}
\hline Material & Tube & Fracture \\
\hline Newtonian & $8 \eta \frac{q}{\pi} \frac{1}{R^{4}}$ & $\frac{12 \eta q}{h} \frac{1}{w^{3}}$ \\
\hline 1X gel & $101 q^{0.26} \frac{1}{R^{1.78}}$ & $156\left(\frac{q}{h}\right)^{0.26} \frac{1}{w^{1.52}}$ \\
\hline 2 X gel & $532 q^{0.21} \frac{1}{R^{1.63}}$ & $760\left(\frac{q}{h}\right)^{0.21} \frac{1}{w^{1.42}}$ \\
\hline 3 X gel & $1300 q^{0.19} \frac{1}{R^{1.57}}$ & $1796\left(\frac{q}{h}\right)^{0.19} \frac{1}{w^{1.38}}$ \\
\hline
\end{tabular}

During extrusion experiments, Seright ${ }^{23,72,73}$ noted that the pressure gradient varied inversely with the square of the fracture width. In examining the gel entries for Table 7, the exponents ranged from 1.57 to 1.78 for the $R$ variables and from 1.38 to 1.52 for the $w$ variables. These exponents were intermediate between the exponent of one (predicted from a force balance with Newtonian fluids) and two (the approximate value observed experimentally).

Pressure gradients versus fracture width were plotted (Fig. 28) for the $1 \mathrm{X}$ gel flowing through fractures at a fixed volumetric rate of $16,000 \mathrm{ml} / \mathrm{hr}$. The pressure gradient values estimated by the models were greater than those from the simple force balance approach. For aperture widths less than $2.54 \mathrm{~mm}(0.1 \mathrm{in})$, the model's calculated pressure gradients more closely matched the extrusion data than calculations from the simple force balance approach. The model described the relation between pressure gradient and aperture size fairly well as long as the aperture was not too wide (e.g., not greater than $2.54 \mathrm{~mm}$ ).

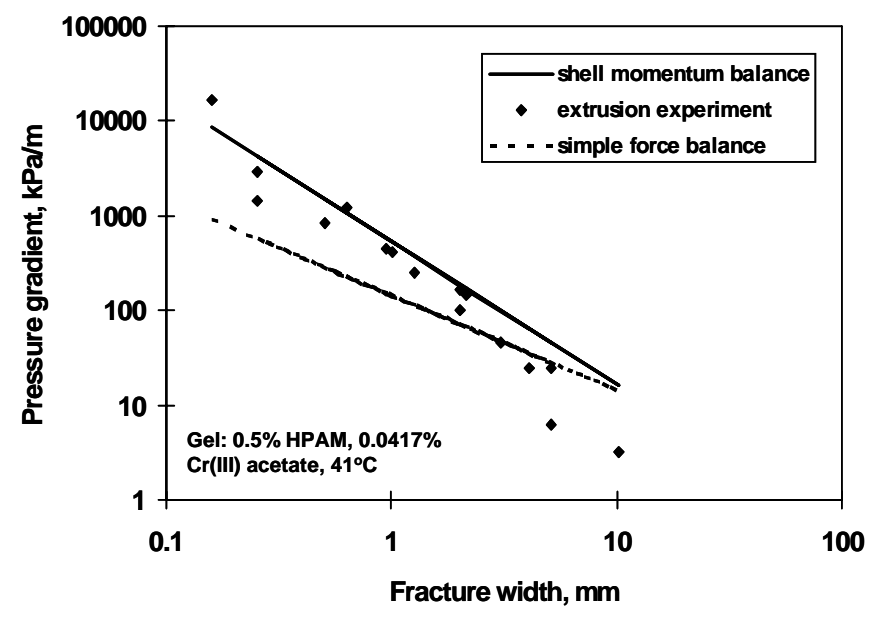

Fig. 28 - Comparison of pressure gradients versus aperture for $1 \mathrm{X}$ gel.

We also calculated the velocity distribution versus aperture for the $1 \mathrm{X}, 2 \mathrm{X}$, and $3 \mathrm{X}$ gels flowing through a $38.1-\mathrm{mm}$ high by $1-\mathrm{mm}$ wide fracture, and a tube with inside diameter of $2 \mathrm{~mm}$ with a flow rate of $16,000 \mathrm{ml} / \mathrm{hr}$. Fig. 29 indicates that the gels moved more like a piston through the apertures than did the Newtonian fluid. 

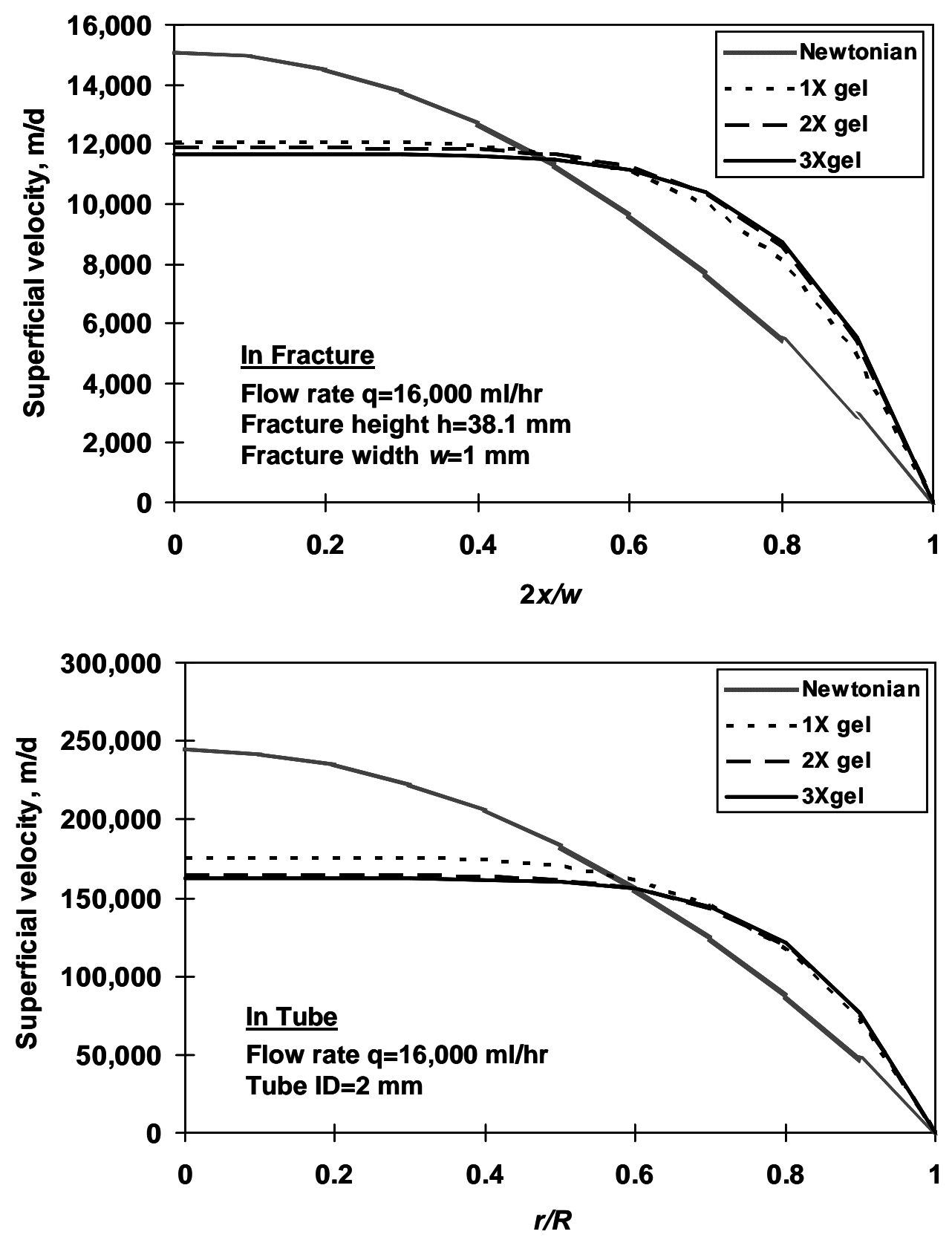

Fig. 29-Velocity distribution for $1 \mathrm{X}, 2 \mathrm{X}$, and $3 \mathrm{X}$ gels.

Pressure Gradient versus Flow Rate and Shear Stress versus Shear Rate. During steady shear measurements, $\mathrm{Liu}^{82,83}$ found that for the $1 \mathrm{X} \mathrm{Cr}(\mathrm{III})$-acetate-HPAM gel, shear stress was fairly insensitive to shear rate (Fig. 30). When the shear rate was increased by a factor of $10^{5}$, the shear stress increased only by a factor of 10 . This result was consistent with Seright's findings that the pressure gradient required to extrude gel through a fracture was fairly insensitive to injection velocity. A log-log plot of complex viscosity versus shear rate gave a slope of -0.8 , which was close to the slopes $(-0.83$ to -0.95$)$ for a log-log plot of resistance factor (apparent viscosity relative to water) versus injection velocity that were noted during gel extrusion through fractures. ${ }^{92,93}$ 


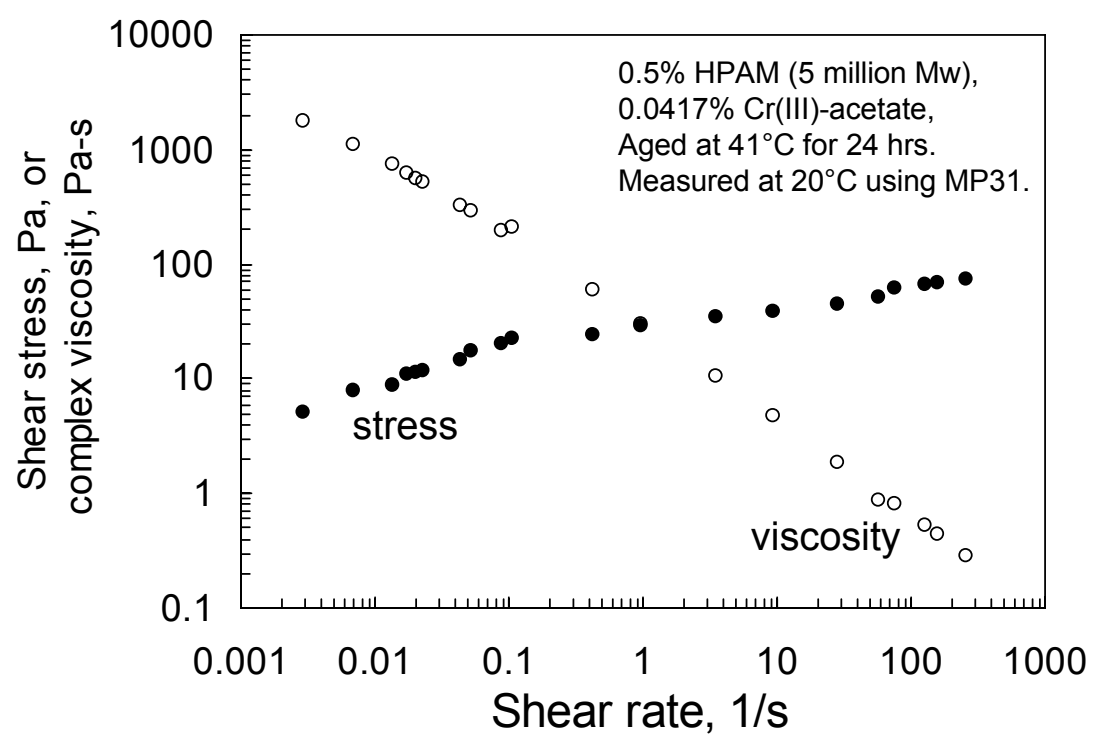

Fig. 30 - Stress and complex viscosity versus shear rate. . $^{82}$

This interesting similarity made us consider the relationship of gel behavior in fractures versus in a rheometer. Combining Eqs. 17, 18, and 20, relates shear rate to the flow rate for Newtonian fluids in tubes:

$$
\dot{\gamma}=-\frac{4 q}{\pi R^{4}} r
$$

Combining Eqs. 17, 23, and 25, gives the shear rate for power-law, non-Newtonian materials in tubes:

$$
\dot{\gamma}=-\frac{3 n+4}{n+1} \frac{q}{\pi} R^{-\frac{3 n+4}{n+1}} \frac{1}{n+1}
$$

Combining Eqs. 18, 27, and 29, gives the shear rate for Newtonian materials in fractures:

$$
\dot{\gamma}=-\frac{12 q}{h w^{3}} x
$$

Combining Eqs. 23, 27, and 32, gives the shear rate for power-law, non-Newtonian materials in fractures:

$$
\dot{\gamma}=-\frac{2 n+3}{n+1} \frac{2 q}{h} w^{-\frac{2 n+3}{n+1}}(2 x)^{\frac{1}{n+1}}
$$

Table 8 shows the correlations of pressure gradient, shear stress, flow rate, and shear rate at the wall, where $r=R$ or $x=w / 2$, for the $1 \mathrm{X}, 2 \mathrm{X}$, and $3 \mathrm{X}$ gels. 
Table 8-Correlation of pressure gradient, shear stress, flow rate, and shear rate at the wall

\begin{tabular}{|c|c|c|}
\hline Material & Tube & Fracture \\
\hline $\begin{array}{c}1 \mathrm{X} \\
\text { gel }\end{array}$ & $\dot{\gamma}=-6.93 \frac{q}{\pi R^{3}}$, & $\dot{\gamma}=-11.86 \frac{q}{h w^{2}}$, \\
\hline $\begin{array}{c}L \\
\text { gel }\end{array}$ & $\dot{\gamma}=-7.69 \frac{p_{0}-p_{L}}{\pi R^{3}}$, & $\tau=\frac{p_{0}-p_{L}}{L} \frac{w}{2}$ \\
\hline $3 \mathrm{X}$ & $\dot{\gamma}=-8.14 \frac{p_{0}-p_{L}}{2} \frac{R}{2}$, & $\tau=\frac{p_{0}-p_{L}}{L} \frac{w}{2}$ \\
gel & $\tau=\frac{p_{0}-p_{L}}{L} \frac{R}{2}$ & $\dot{\gamma}=-14.28 \frac{q}{h w^{2}}$, \\
\hline
\end{tabular}

Comparison in Tubes. Based on pressure gradients and flow rates from the extrusion experiments, we calculated shear stresses and shear rates for the $1 \mathrm{X}, 2 \mathrm{X}$, and $3 \mathrm{X}$ gels using the equations in Table 8. These calculations were compared to the rheology measurements made with a gap height of $1 \mathrm{~mm}$ on smooth and rough surfaces. In tubes (Fig. 31 for the case of a 1X gel), the calculated shear stress versus shear rate relation followed the same trend as the rheology measurements for shear rates above $100 \mathrm{~s}^{-1}$. However, the measured shear stresses were higher than the calculated values for tube flow for shear rates below $100 \mathrm{~s}^{-1}$. More work is needed to understand this behavior.

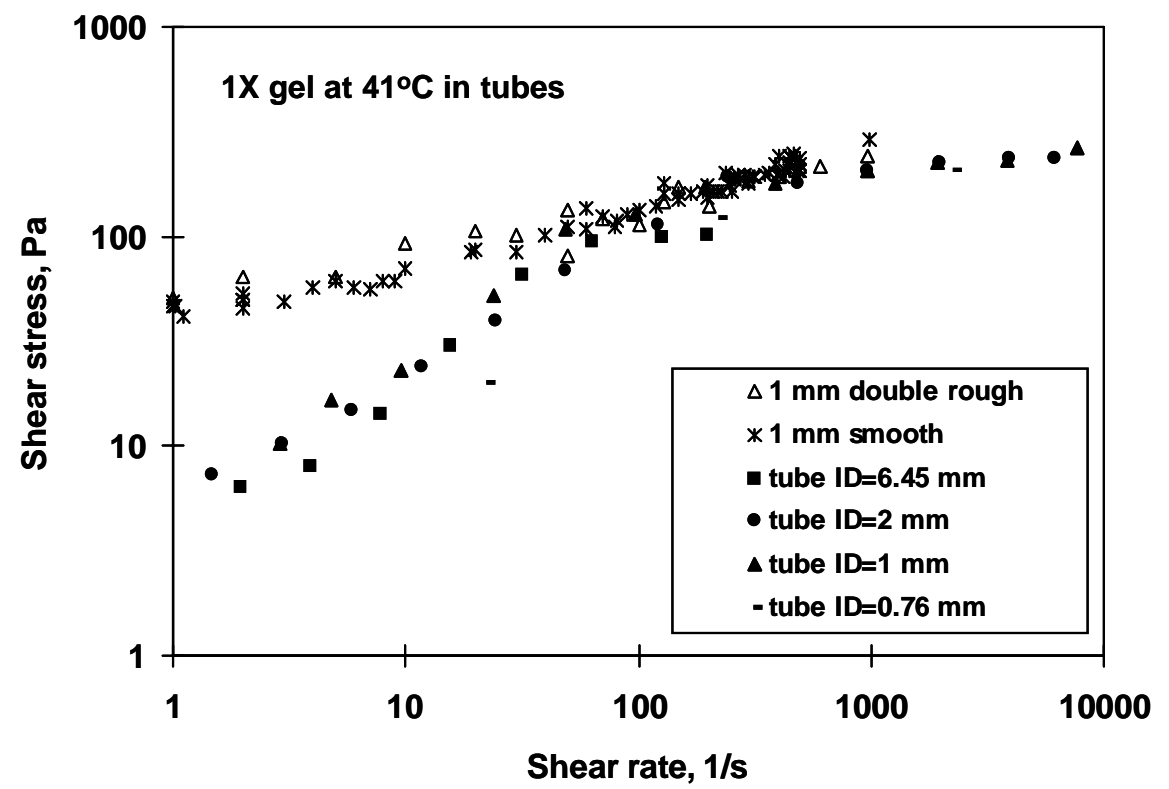

Fig. 31-Comparison of shear stress versus shear rate for $1 \mathrm{X}$ gel in tubes. 
Comparison in Fractures. For a 2-mm wide fracture (Fig. 32 for the case of a $1 \mathrm{X}$ gel), the calculated shear stress versus shear rate relation followed the same trend as the rheology measurements. However, for narrower fractures $(0.254 \mathrm{~mm}$ and $1 \mathrm{~mm})$, shear stress values calculated from the extrusion experiments were generally much higher than the rheology measurements. The highest shear stress values were noted in the narrowest fracture. This discrepancy could be explained by the fairly rough Berea sandstone surface seriously interfering with gel flow in the fracture, especially for very narrow fractures. For wider fractures (e.g., 2 $\mathrm{mm}$ ) this interference became negligible; thus, the flow curve was similar to our rheology measurements. The similarity between the flow curves from the fracture flow or tube flow and the rheology measurements helps to bridge the gap between gel behaviors in fractures or tubes with those seen in a rheometer. However, additional work is needed to fully explain the high pressure gradients observed during gel extrusion through narrow fractures.

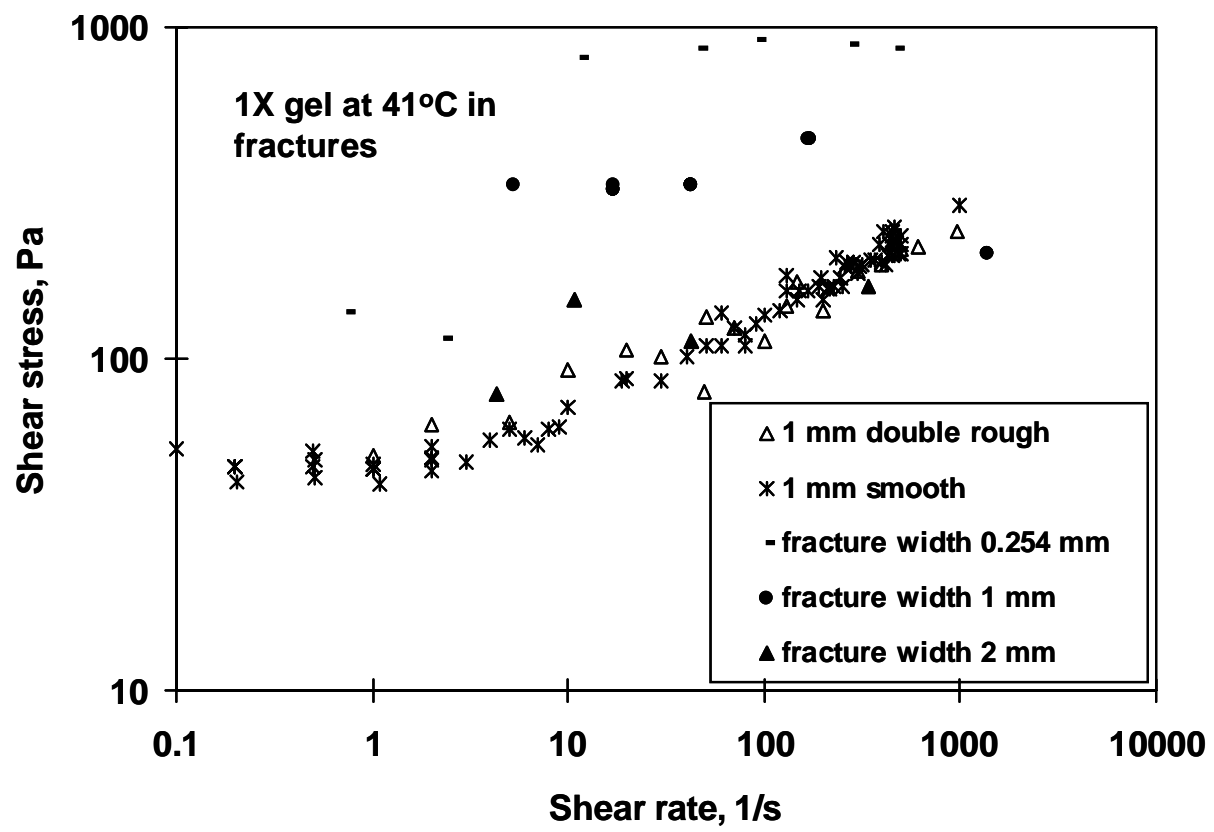

Fig. 32-Comparison of shear stress versus shear rate for $1 \mathrm{X}$ gel in fractures.

\section{Conclusions}

When evaluating the use of gels for conformance improvement in treating channeling through fractures, rheology measurements can be made much faster and with much lower cost than measurements made during extrusion of gels through fractured cores. However, the pressure gradient required to extrude a gel through a fracture is usually substantially greater than anticipated based on rheology measurements combined with a simple force balance. This chapter examined this discrepancy, in hopes of ultimately substituting the rheology measurements for extrusion experiments. The following conclusions were reached, based on studies of $\mathrm{Cr}(\mathrm{III})$ acetate-HPAM gels at $41^{\circ} \mathrm{C}$. 
1. Use of double-rough, parallel plates in our rheometer significantly reduced the importance of wall slip (compared to smooth plates). However, the higher stresses noted during the absence of slip effects were not enough to explain the high pressure gradients during gel extrusion experiments.

2. Consistent with expectations, the first normal stress difference increased with shear rate and polymer concentration for our $\mathrm{Cr}(\mathrm{III})$-acetate-HPAM gels. Although first normal stress might result in greater pressure gradients during extrusion through fractures with significant constrictions, it was not great enough to explain the high pressure gradients during extrusion experiments.

3. Earlier work revealed that gels propagate through fractures by wormholing through immobile concentrated (dehydrated) gel. Since these wormholes are narrower than the fracture width, this could partly explain the higher pressure gradients during gel extrusion experiments.

4. A model based on power-law and shell momentum balance predicted that pressure gradient should vary with fracture width raised to a power between -1 and -2 . This model fit our extrusion data reasonably well for fracture widths of $1 \mathrm{~mm}$ or less.

5. In 2-mm wide fractures, a second model did well in correlating pressure gradient with shear stress and flow rate with shear rate. However, in narrower fractures, the model was notably less successful. Additional work is needed to fully explain the high pressure gradients observed during gel extrusion through narrow fractures. 


\section{SWEEP IMPROVEMENT OPTIONS FOR THE DAQING OIL FIELD}

\section{Introduction}

Laboratory research began in the 1960s, investigating the potential of enhanced oil recovery (EOR) processes in the Daqing Oil Field. Use of polymer flooding was identified as a key method to improve areal and vertical sweep efficiency, as well as providing mobility control. ${ }^{106}$ Consequently, the world's largest polymer flood was implemented at Daqing, beginning in 1996. ${ }^{107,108}$ By 2004, 22.7\% of total production from the Daqing Oil Field was attributed to polymer flooding. Polymer flooding should boost the ultimate recovery for the field to over 50\% original oil in place (OOIP) - 10\% OOIP more than from waterflooding.

Alkaline/surfactant/polymer flooding (ASP) has also experienced extensive laboratory testing in China. The ASP technique was gradually perfected for application at Daqing and has been pilot tested on a large scale. ${ }^{109-111}$ Results from two typical pilot tests in the south and north parts of the Daqing field revealed incremental oil recovery values as high as $20 \%$ OOIP. By the end of 2003, the Center Xinger pilot site showed a definitive increase in oil production and decrease in water cut. In the east area of this site (the largest portion included within the ASP pattern area), the predicted EOR was about $18 \%$ OOIP more than from waterflooding.

Although field tests of polymer and ASP flooding have been very successful at Daqing, concerns about sweep efficiency persist-particularly the possibility of channeling expensive chemical formulations through the reservoir. Therefore, the sweep efficiency at Daqing is receiving intense scrutiny, and possibilities for improvement are being considered. In this chapter, we examine the nature of reservoir sweep in parts of the Daqing Oil Field that have been EOR targets. We investigate the potential of various approaches for improving sweep, including (1) gel treatments that are directed at channeling through fractures, (2) colloidal dispersion gels, (3) reduced polymer degradation, (4) more viscous polymer solutions, and (5) foams and other methods (e.g., thermal and microbial techniques). Our objective is to establish better options for improving sweep in the main producing zone in the future.

\section{Sweep Efficiency in the Daqing Oil Field}

Stratification. What is the nature of the sweep efficiency problem at Daqing? This study focused on the Eastern Berxi polymer flooding area of the Daqing field-specifically on 17 production wells and 8 injection wells. Layer PI, a principal target for EOR at Daqing, is composed of up to seven named "zones." The gross pay for Layer PI varied between 18 and 33 meters. Net pay varied from 11 and 31 meters, averaging 18 meters. Within the net pay, some degree of vertical stratification existed. Figs. 33-35 illustrate this stratification for three wells. For the 25 wells, the Dykstra-Parsons coefficient of permeability variation ranged from 0.14 to 0.89, averaging 0.70. Fig. 33 illustrates the distribution for Production Well B2-4-P43. In this well, five strata were identified, with the top zone being the most permeable zone $\left(1.2 \mu \mathrm{m}^{2}\right)$. Three intermediate zones had about the same permeability, ranging from 0.4 to $0.5 \mu \mathrm{m}^{2}$. A small zone at the bottom was the least permeable, with $0.232 \mu \mathrm{m}^{2}$. For this well, most flow capacity and net pay existed in a single interval where crossflow could freely occur. This feature was shared by $32 \%$ of the wells examined. 


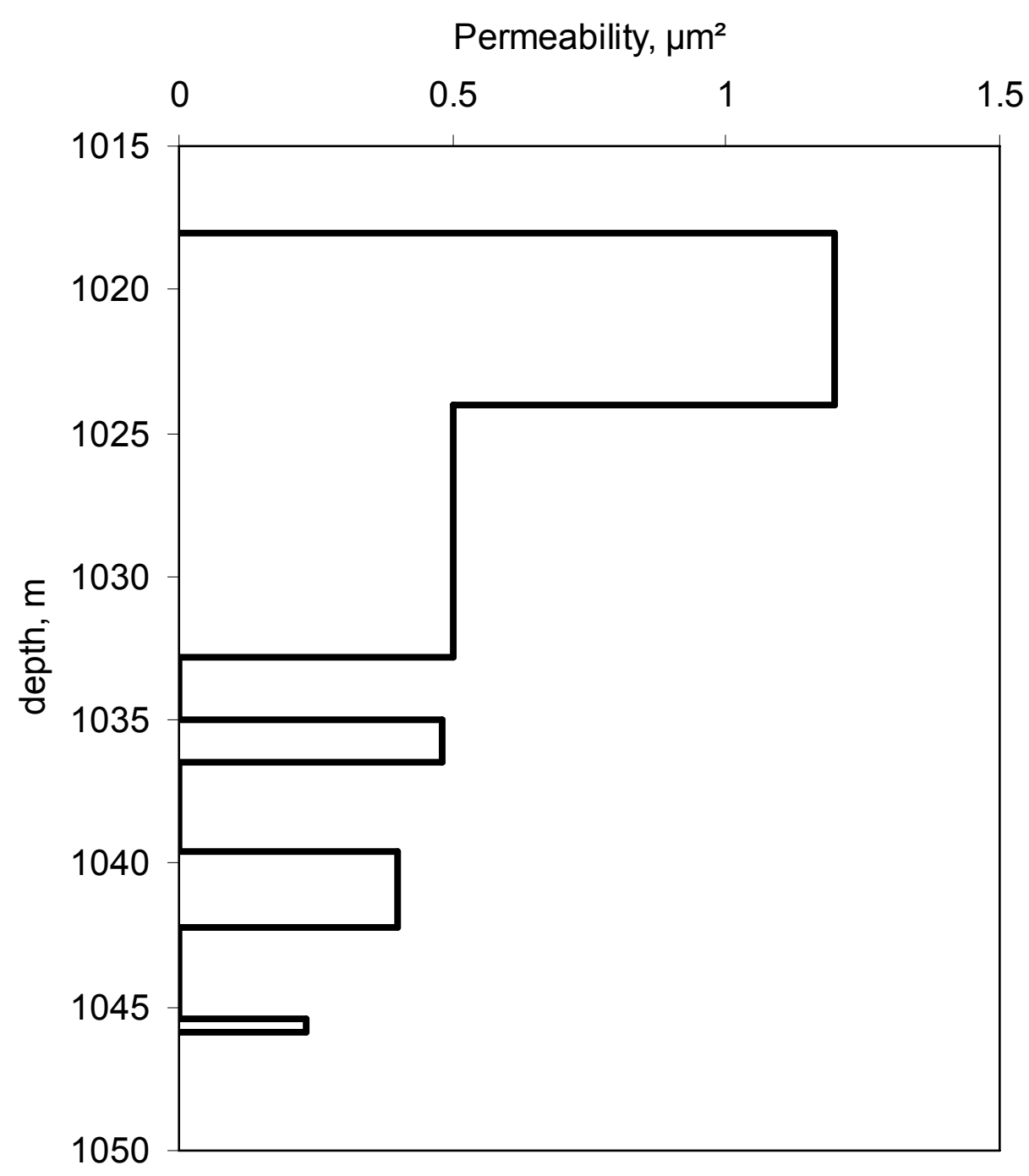

Fig. 33-Permeability distribution Producer B2-4-P43.

Fig. 34 illustrates the distribution for Production Well B2-D6-P45. Four strata were identified, with the top and bottom zones having similar permeabilities $\left(0.49-0.5 \mu \mathrm{m}^{2}\right)$. A $0.316-\mu \mathrm{m}^{2}$ zone at $1,017 \mathrm{~m}$ probably had direct flow contact (crossflow) with the bottom zone. A small 0.142 $\mu \mathrm{m}^{2}$ zone at $1,010 \mathrm{~m}$ appeared distinct from the other zones. For this well, most of the net pay existed in zones that had about the same permeability. This feature was exhibited by $36 \%$ of the wells examined.

Fig. 35 illustrates the distribution for Production Well B2-D6-P47, where seven strata were identified. The interval from 988 to $1,001 \mathrm{~m}$ should be considered as a single flow unit, with an average permeability around $0.5 \mu \mathrm{m}^{2}$. A $1.2-\mu \mathrm{m}^{2}$ zone existed at $1,017 \mathrm{~m}$. Between 1,005 and $1,013 \mathrm{~m}$, three small zones were present, with permeabilities ranging from 0.147 to $0.21 \mu \mathrm{m}^{2}$. Thus, for this well, the net pay existed in distinct zones with noticeably different permeabilities. This characterization was shared by $28 \%$ of the wells examined. 


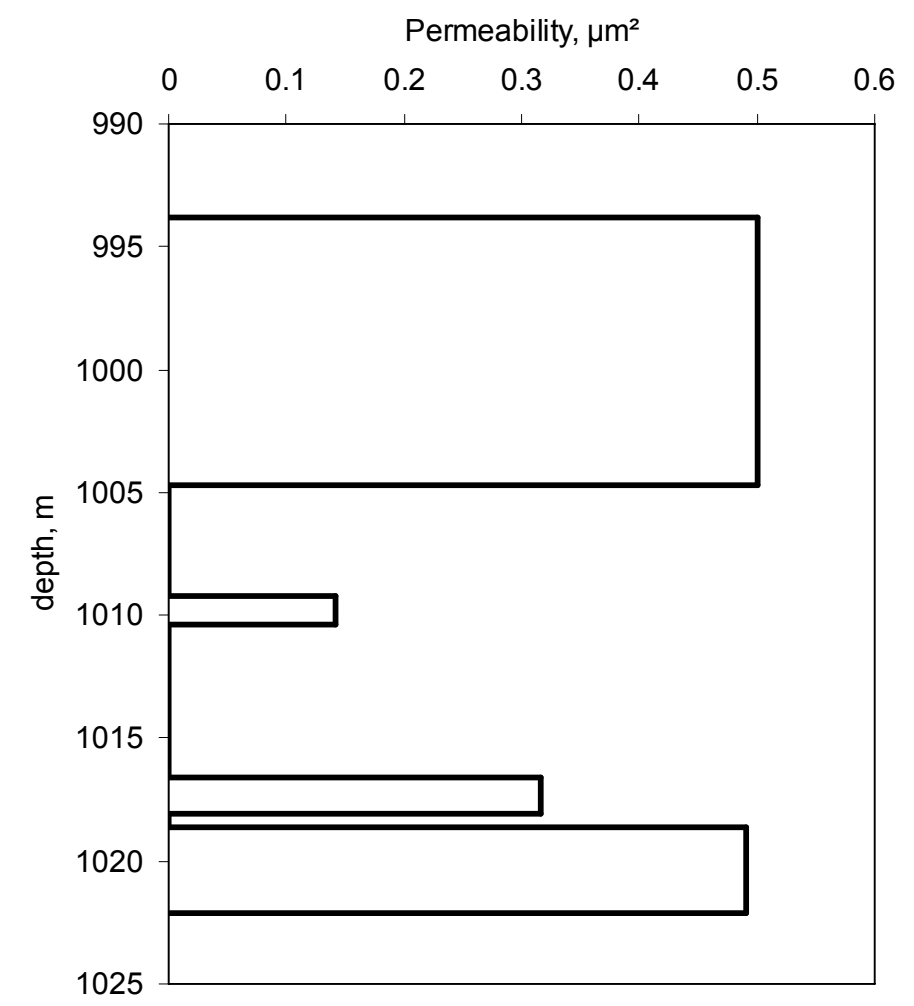

Fig. 34-Permeability distribution Producer B2-D6-P45.

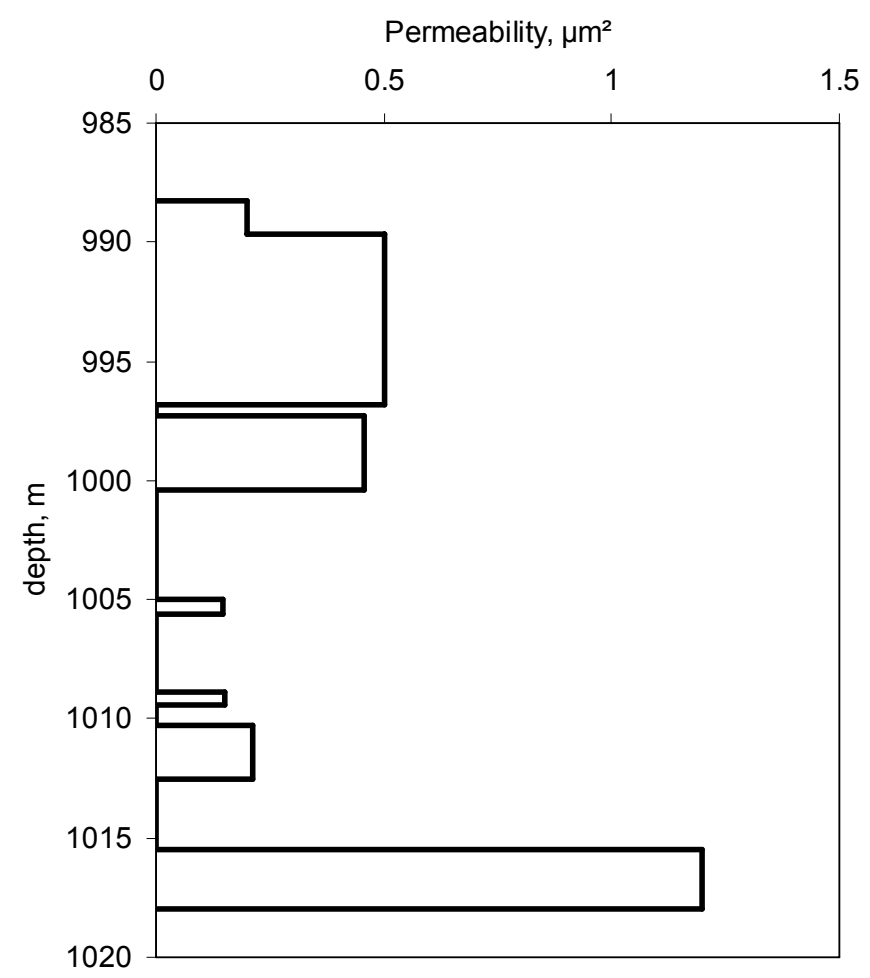

Fig. 35-Permeability distribution Producer B2-D6-P47. 
Mobility Ratios. At Daqing, the endpoint relative permeabilities were $k_{r w} \sim 0.5$ and $k_{r o} \sim 0.8$, oil viscosity was $9 \mathrm{mPa}-\mathrm{s}$, and water viscosity was $0.6 \mathrm{mPa}$-s. Thus, during waterflooding, the endpoint mobility ratio was 9.4. With this unfavorable mobility ratio, viscous fingers can form that lead to severe channeling, especially when zones with different permeability are present.

By injecting a viscous polymer solution, the mobility ratio was increased to the point of making it favorable. The viscosity of the injected polymer solution was typically 35 to $40 \mathrm{mPa}$-s. If polymer degradation was not significant, this level of viscosity decreased the mobility ratio from 9.4 to about 0.25 . When fluids can freely crossflow between strata, the rate of movement of a polymer front is independent of permeability, so long as the reciprocal of the mobility ratio is greater than the permeability contrast between the strata. ${ }^{12}$ The two upper strata in Fig. 33 had permeabilities of 0.5 and $1.2 \mu \mathrm{m}^{2}$ (i.e., a permeability contrast of 2.4 and combined average permeability of $0.783 \mu^{2}$ ) and were subject to unrestricted crossflow. Thus, during polymer flow where the reciprocal mobility ratio was 4 (i.e., 1/0.25), the upper strata in Well B2-4-P43 should have been swept quite efficiently.

The bottom three strata in Fig. 33 had permeabilities of $0.482,0.398$, and $0.232 \mu \mathrm{m}^{2}$, respectively. The relative rates of propagation for the polymer fronts in each stratum can be estimated using the methods in Refs. 13, 17, and 112. Given the permeabilities and the above mobility ratio, the relative rates of propagation of the polymer fronts for radial flow in the five zones would be $1,1,0.79,0.72$, and 0.56 , respectively. Some evidence exists that the polymer may experience up to $50 \%$ loss of viscosity during injection. If the reciprocal mobility ratio was 2 instead of 4 , the relative rates of propagation of the polymer fronts in the five zones were 1,1 , $0.78,0.71$, and 0.54 , respectively.

For the above circumstances, sweep efficiency during the polymer flood should be quite good, with the possible exception of the very small $0.232-\mu \mathrm{m}^{2}$ bottom zone. Similar calculations can be made for the wells illustrated in Figs. 34 and 35. These calculations reveal that the polymer flood should have provided excellent sweep in all but the small zone at 1,010 $\mathrm{m}$ for Well B2-D6-P45 (Fig. 34) and the small zones from 1,005 to 1,013 $\mathrm{m}$ in Well B2-D6-P47 (Fig. 35). More generally, these calculations indicated that the polymer flood should have provided excellent sweep throughout the vast majority of the patterns associated with the 25 wells under consideration. Thus, if sweep was truly inadequate during the polymer flood, a reason for this inadequacy must be identified.

\section{Mobility Control during ASP Flooding}

ASP flooding is being considered to increase recovery efficiency from the Daqing Oil Field. Mobility control and sweep improvement will be especially important during implementation of the ASP process.

Mobility Control Requirements for ASP Flooding after Polymer Flooding. During a chemical flood (e.g., an ASP flood), mobility control means each successive fluid bank injected must be less mobile than the bank ahead of it. Without mobility control, expensive chemical banks experience significant channeling (via viscous fingers) even in homogeneous reservoirs. In heterogeneous reservoirs, channeling is accentuated with unfavorable mobility ratios. 
If an ASP flood is the first EOR method applied in a reservoir, the ASP bank should be less mobile (more viscous) than the oil/water bank that is being displaced. Because an ASP formulation decreases the residual oil saturation, effective permeability to the aqueous phase (i.e., the ASP formulation) can be much greater than that in a preceding waterflood. Consequently, the level of viscosity enhancement (mobility reduction) needed to provide mobility control is significantly greater than that if no reduction in $S_{o r}$ occurred.

For some areas of the Daqing Oil Field, ASP floods may be applied in patterns that were previously polymer flooded. Since the preceding polymer bank was quite viscous (20-40 mPa-s), the ASP bank must be even more viscous to maintain mobility control.

Higher Salinity of ASP Reduces Viscosity. Compounding this mobility problem, alkaline agents significantly increase the ionic strength of ASP formulations. The viscosity of a given concentration of anionic polymer (e.g., HPAM) is much lower in saline solutions (e.g., an ASP formulation) than in fresh water (e.g., used during the Daqing polymer floods). ${ }^{2-6}$ Thus, the concentrations and expense for polymer may be unusually high when applying ASP flooding after a polymer flood.

Figs. 36 and 37 show viscosity versus polymer concentration for polymers with medium and ultra-high molecular weights. As expected, viscosity increased with the increased polymer concentration, and was significantly lower with $1.4 \%-1.5 \%$ alkali than without alkali.

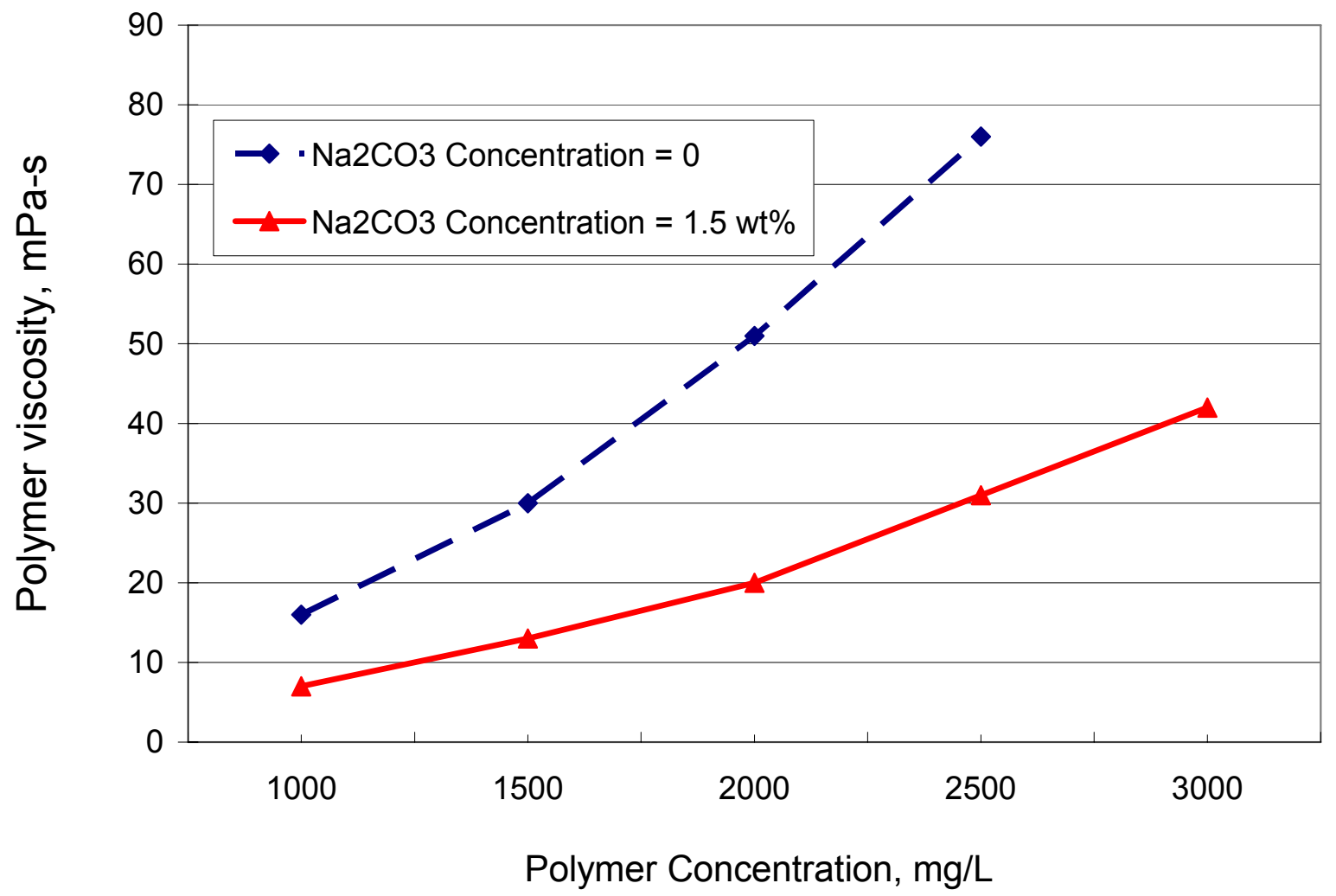

Fig. 36-Viscosity of medium molecular weight polymer (LIANHUA-1500). 


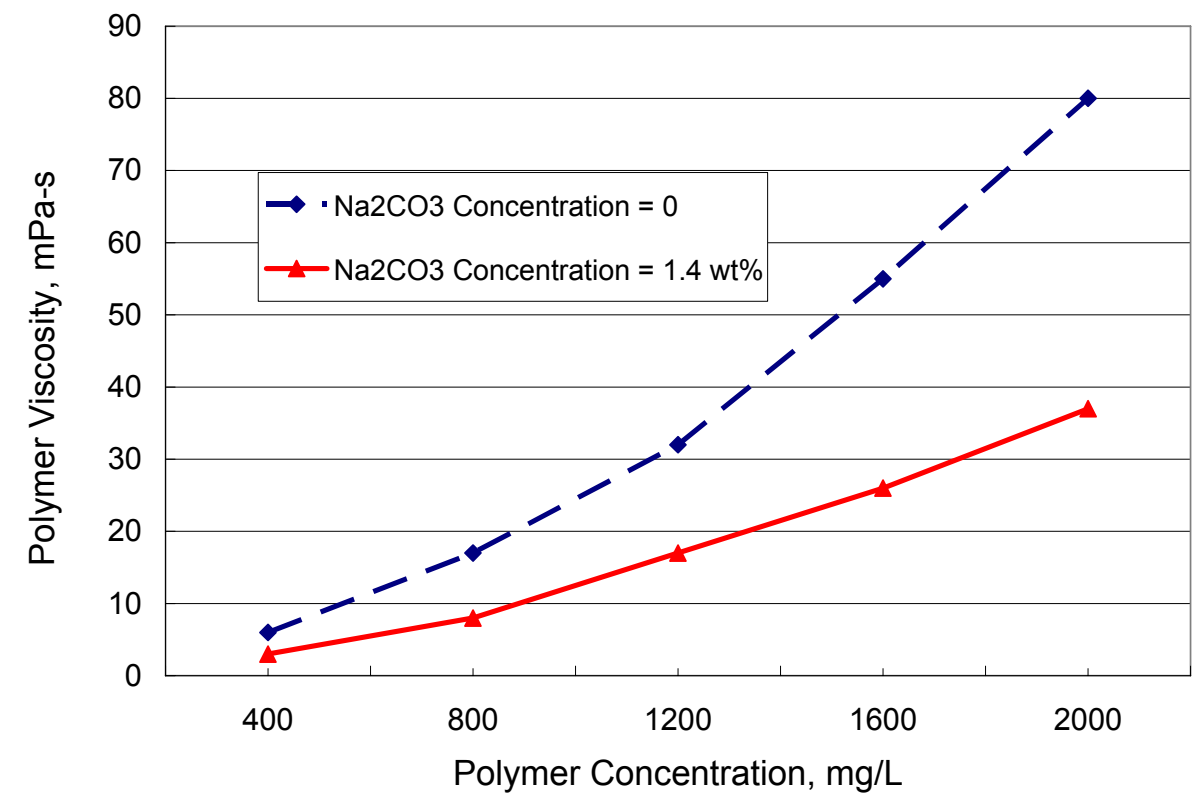

Fig. 37-Viscosity of ultra-high molecular weight polymer (HENGJU).

Polymer Stability in the Presence of Alkali. When HPAM solutions are exposed either to high temperatures or high $\mathrm{pH}$ values, the polymer's amide groups can hydrolyze. ${ }^{113,114}$ However, the carbon-carbon backbone of the polymer is quite stable, as long as oxygen and free radials are absent. Fig. 38 shows the stability of an ASP solution when the alkali concentration is $1.2 \%$. The curve demonstrates that the ASP viscosity can be kept stable for at least 3 months when the polymer concentration is $1,200 \mathrm{mg} / \mathrm{L}$.

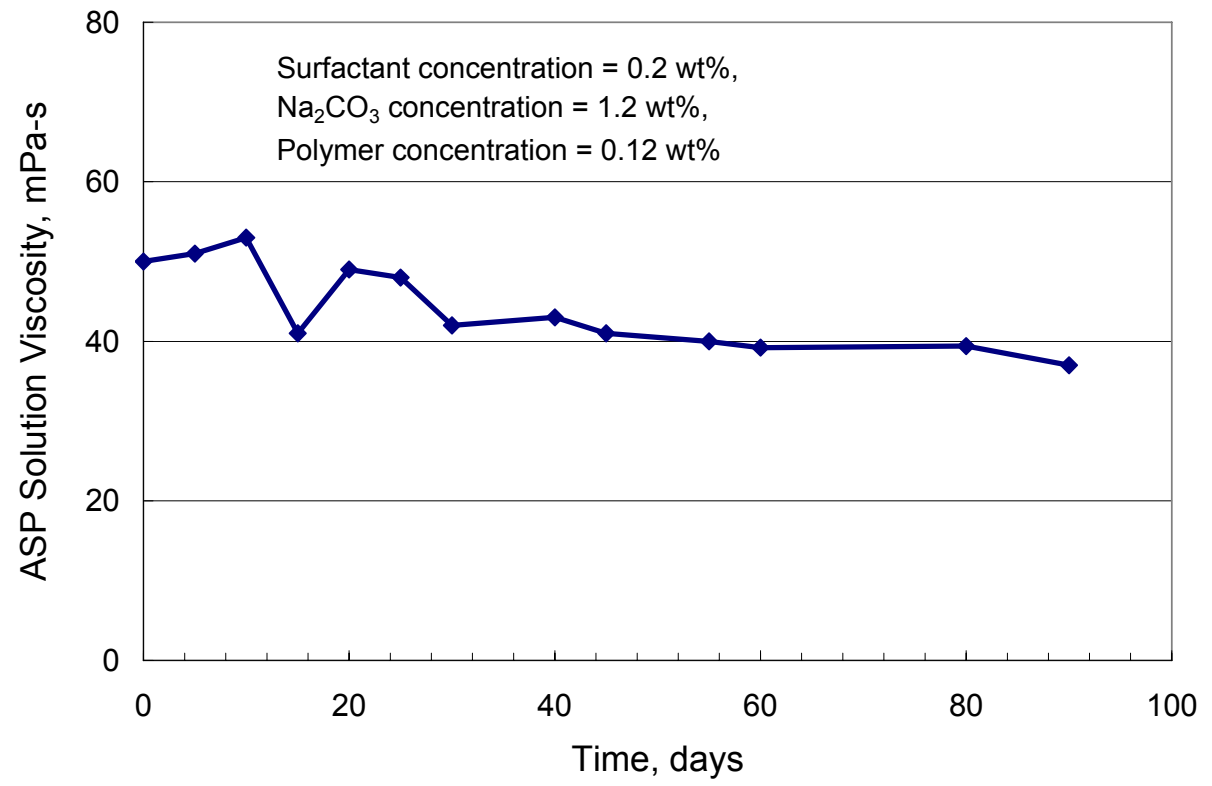

Fig. 38 - Stability of ASP solution viscosity. 
Reduced Injectivity for Viscous Solutions. ASP flooding displaces residual oil and increases the effective permeability, especially near injection wells. This effect enhances injectivity index. However, this beneficial effect is countered by the increased viscosity, reduced mobility and/or any permeability reduction associated with polymer in the ASP formulation. ${ }^{111}$

Figs. 39 and 40 show the predicted variation of injection pressures (based on the numerical simulation and the real data from the pilot site) before versus after ASP flooding in the First North-Fault from the West pilot test. Injection pressures increased gradually during injection of ASP solution. After 0.23 pore volumes $(P V)$ of ASP, the average injection pressure increased from $9.18 \mathrm{MPa}$ (during water drive) to $12.47 \mathrm{MPa}$ - an increase of 3.29 MPa. Here, the injection rate was $0.206 \mathrm{PV} /$ year, and the ASP injection viscosity was $37 \mathrm{mPa}$-s. Consequently, the injection ability and flow pressure decreased, but the injection pressure was still $1.03 \mathrm{MPa}$ below the formation fracture pressure. At the peak predicted injection pressure, the water injectivity index for ASP was $13.9 \%$ less than during water injection. Even so, this loss of injectivity was less than that observed during polymer flooding, see Tables 9 and 10.

Table 9-Variation of injection pressure

\begin{tabular}{|c|c|c|c|c|c|}
\hline \multirow{2}{*}{ Method } & \multirow{2}{*}{$\begin{array}{l}\text { Well } \\
\text { distance } \\
(\mathrm{m})\end{array}$} & \multirow{2}{*}{$\begin{array}{l}\text { Injection } \\
\text { intensity } \\
\left(\mathrm{m}^{3} / \mathrm{d} \cdot \mathrm{m}\right)\end{array}$} & \multicolumn{2}{|c|}{$\begin{array}{c}\text { Injection pressure } \\
(\mathrm{MPa})\end{array}$} & \multirow{2}{*}{$\begin{array}{l}\text { Pressure increase } \\
\qquad(\mathrm{MPa})\end{array}$} \\
\hline & & & $\begin{array}{l}\text { water } \\
\text { drive }\end{array}$ & $\begin{array}{c}\text { chemical } \\
\text { flood }\end{array}$ & \\
\hline ASP & 250 & 20.5 & 9.18 & 12.47 & 3.29 \\
\hline Polymer & 250 & 17.3 & 5.5 & 12.3 & 6.8 \\
\hline
\end{tabular}

Table 10 - Variation of water injectivity index

\begin{tabular}{|c|c|c|c|c|c|}
\hline \multirow{2}{*}{ Method } & \multirow{2}{*}{$\begin{array}{l}\text { Well } \\
\text { distance } \\
(\mathrm{m})\end{array}$} & \multirow{2}{*}{$\begin{array}{l}\text { Injection } \\
\text { intensity } \\
\left(\mathrm{m}^{3} / \mathrm{d} \cdot \mathrm{m}\right)\end{array}$} & \multicolumn{2}{|c|}{$\begin{array}{l}\text { Water injectivity index } \\
\left(\mathrm{m}^{3} / \mathrm{d} \cdot \mathrm{m} \cdot \mathrm{MPa}\right)\end{array}$} & \multirow{2}{*}{$\begin{array}{l}\text { Pressure increase } \\
\quad(\mathrm{MPa})\end{array}$} \\
\hline & & & $\begin{array}{l}\text { water } \\
\text { drive }\end{array}$ & $\begin{array}{l}\text { chemical } \\
\text { flood }\end{array}$ & \\
\hline ASP & 250 & 20.5 & 3.51 & 3.02 & 3.29 \\
\hline Polymer & 250 & 17.3 & 2.75 & 2.08 & 6.8 \\
\hline
\end{tabular}




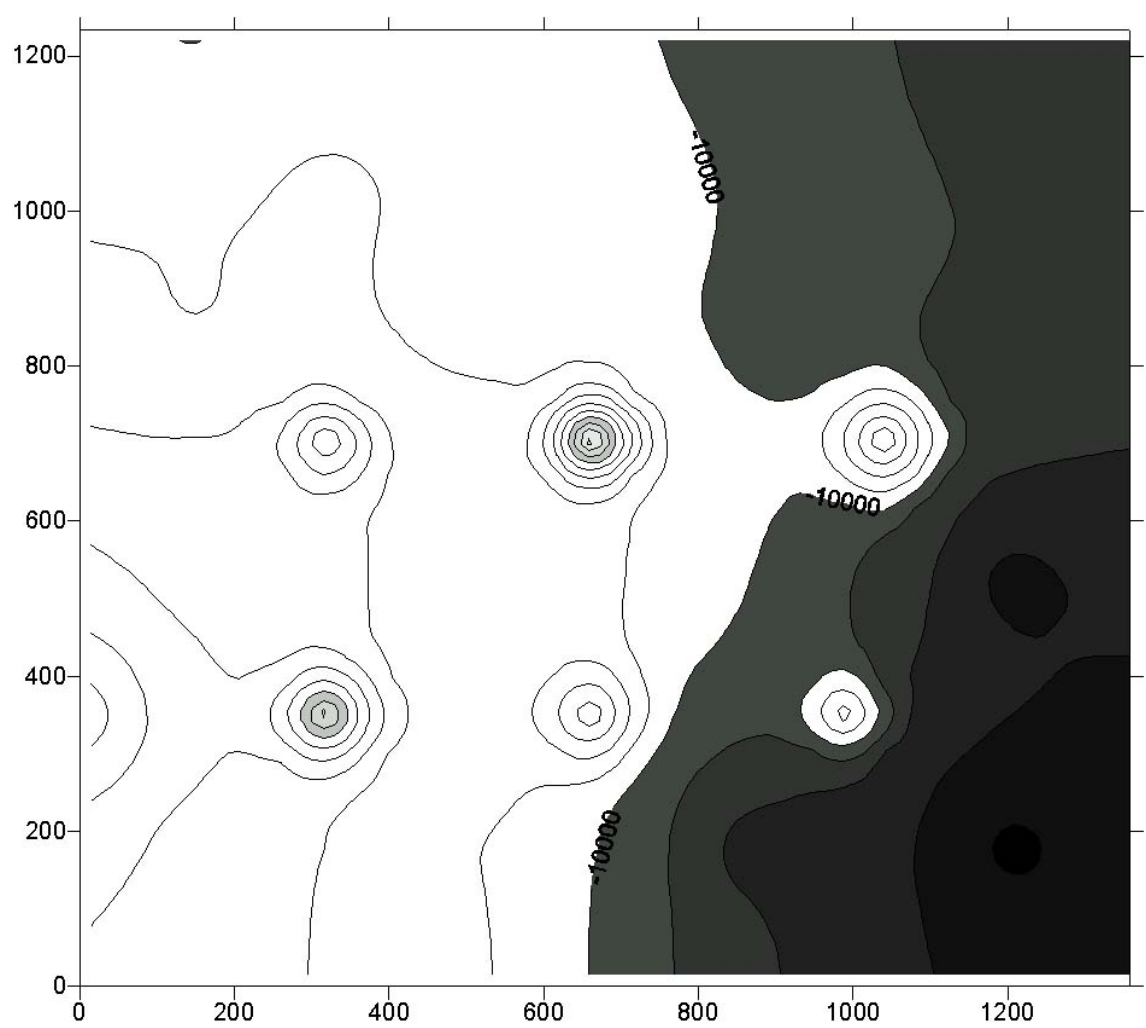

Fig. 39-Pattern pressures before ASP: Boundary is $10 \mathrm{MPa}$.

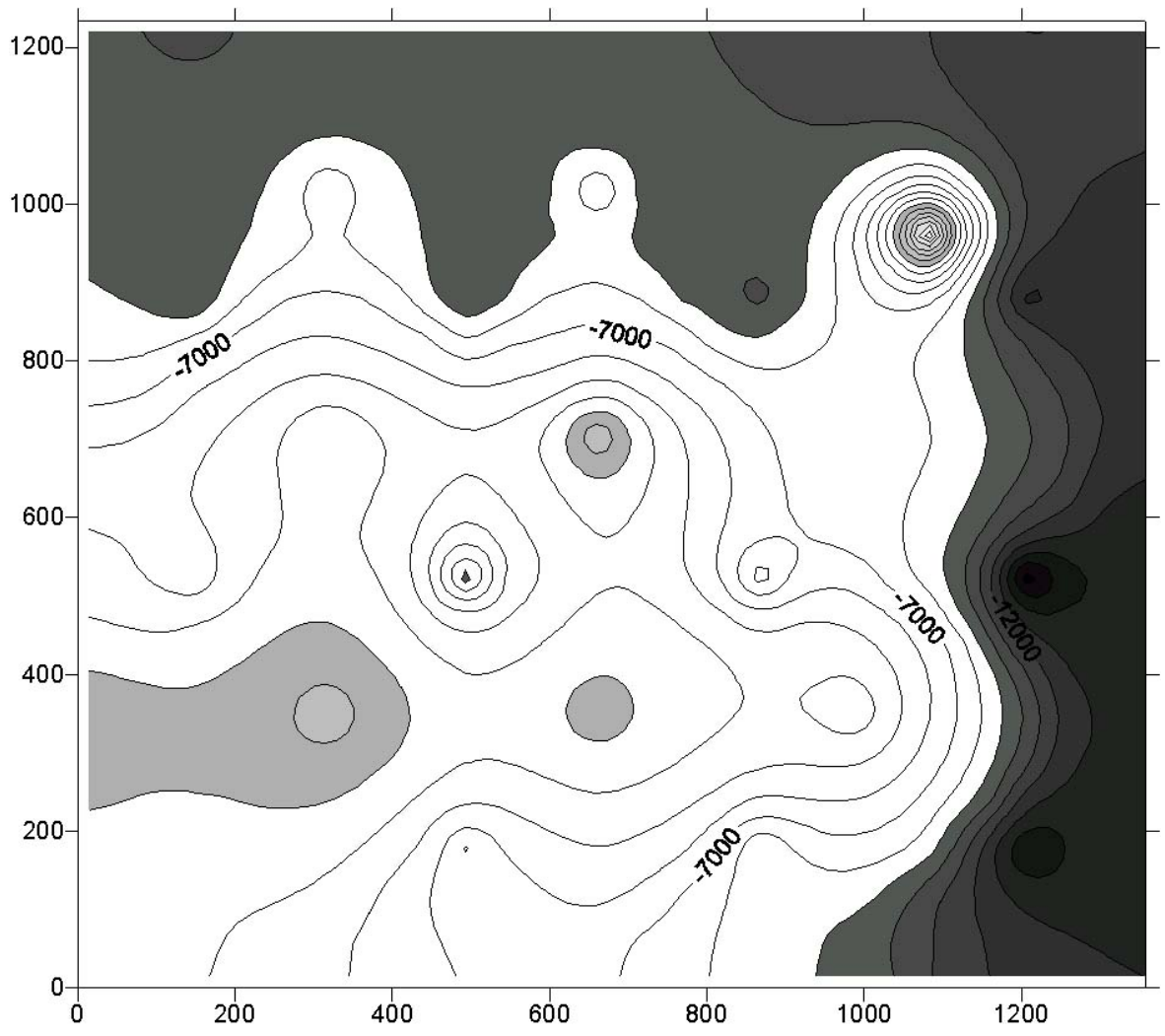

Fig. 40-Pattern pressures after ASP: Boundary is $12 \mathrm{MPa}$. 


\section{Are Fractures Important? Are Gels Viable to Reduce Channeling?}

We examined whether fractures might cause significant channeling of polymer between some injector-producer pairs at Daqing. Typically, around 2,000 wells were intentionally hydraulically fractured each year at Daqing. Unintentional fracturing of injection wells is a possibility during waterflood operations. Also, natural fractures may exist. If fractures are responsible for significant channeling between wells, gel treatments could be a viable solution. If fractures are not responsible for channeling, other sweep improvement options may be more appropriate.

In assessing whether fractures cause channeling, we considered three sets of data: (1) pattern oil recovery factors, (2) injectivity and productivity indexes, and (3) inter-well polymer breakthrough times.

Pattern Oil Recovery Factors. In examining our 25 wells, oil recovery factors were generally quite high for individual pattern areas - at least 33\% original oil in place (OOIP) and more typically around 50\% OOIP. Thus, if channeling through fractures was a problem, we must use means other than recovery factors to identify the offending fractures.

Injectivity/Productivity Indexes. One method to assess the importance of fractures is to compare the actual injectivity or productivity index for a well $(q / \Delta p)$ with the value calculated using the Darcy equation for radial flow. ${ }^{44}$

$$
q / \Delta p=\Sigma k h /\left[\mu \ln \left(r_{e} / r_{w}\right)\right]
$$

If the left side of Eq. 37 is substantially greater than the right side, a fracture or fracture-like feature probably intersects the well. On the other hand, if the left side of Eq. 37 is less than or equal to the right side, fractures may not contribute significantly to the flow capacity of the well. We applied this method to our 25 Daqing wells. Most wells showed behavior that was consistent with radial flow (no significant fractures). However, the analysis revealed that fractures were present in three injection wells and three production wells. During polymer flooding, the injectivity or productivity indexes for these wells were typically three to five times greater than values calculated using the right side of Eq. 37.

For those wells where fractures were present, injectivity or productivity indexes can be used to estimate fracture widths $\left(w_{f}\right)$.

$$
\begin{aligned}
& k_{f} w_{f}=\left\{\left[q \mu /\left(\Delta p h_{f}\right)\right]-\left[k_{m} / \ln \left(r_{e} / r_{w}\right)\right]\right\} \quad L_{f} / 2 \ldots \ldots \ldots . . . . \\
& w_{f}(\mathrm{~mm})=1.49\left(k_{f} w_{f}\right)^{1 / 3}, \text { where } k_{f} w_{f} \text { is in } \mu \mathrm{m}^{2}-\mathrm{m}
\end{aligned}
$$

In these equations, $k_{f}$ is effective fracture permeability, $q$ is the total fluid injection or production rate, $\mu$ is fluid viscosity, $\Delta p$ is the well-formation pressure difference, $h_{f}$ is fracture height, $L_{f}$ is fracture half length, $k_{m}$ is effective permeability of the porous rock (average $k_{w}=0.259 \mu \mathrm{m}^{2}$ at $\left.S_{o r}\right), r_{e}$ is external drainage radius $(\sim 150 \mathrm{~m})$, and $r_{w}$ is wellbore radius $(0.1 \mathrm{~m})$. Using Eqs. 38 and 39 and data applicable during polymer injection, calculated fracture widths for the six target wells ranged from 1.4 to $5.0 \mathrm{~mm}$. Interestingly, during water injection after polymer injection, these calculations suggested that the fracture widths ranged from 0 to $1.8 \mathrm{~mm}$. Injection 
pressures were noticeably higher during polymer injection than during the subsequent water injection, so the fractures may have been more open during polymer flooding.

Inter-Well Polymer Breakthrough. The inter-well transit time for a tracer can also be used to assess whether channeling through fractures is important. We estimated the expected transit time $(t)$ for flow through the matrix along the fastest streamline connecting an injection well and a production well. Eq. 40 was used to make this estimate.

$$
t \approx L^{2} \phi\left(1-S_{o r}\right) \mu /\left(k_{m} \Delta p\right)
$$

In this equation, $L$ is the distance between wells (300 $\mathrm{m}$ or $\sim 1,000 \mathrm{ft}$ ), $\phi$ is formation porosity (0.261), $S_{\text {or }}$ is residual oil saturation (0.28), and $\Delta p$ is pressure difference between the wells (20 $\mathrm{MPa}$ or $\sim 2,900 \mathrm{psi}$ ). For most wells examined, the actual polymer breakthrough times (6 to 8 months) were reasonably consistent with the times calculated using Eq. 40, assuming flow through matrix (porous rock). However, for the six wells where injectivity/productivity data suggested the presence of fractures, the actual polymer breakthrough times were noticeably faster than those estimated using Eq. 40. So initially, we viewed this result as confirmation that fractures caused significant channeling between three injector-producer pairs.

If a fracture is dominantly responsible for channeling between two wells, Eq. 41 can be used to estimate the effective permeability of the fracture, and Eq. 42 can convert the effective fracture permeability to an effective average fracture width.

$$
\begin{aligned}
& k_{m} \approx L^{2} \mu /(t \Delta p) \\
& w_{f}(\mathrm{~mm})=3.46 \times 10^{-3}\left(k_{f}\right)^{0.5} \text {, where } k_{f} \text { is in } \mu \mathrm{m}^{2}
\end{aligned}
$$

Applying Eqs. 41 and 42 to our six candidate wells yielded estimates of effective fracture widths around $0.01 \mathrm{~mm}$ - far smaller than the 1- to 5-mm values from Eqs. 38 and 39. How can this discrepancy be explained? A credible possibility is that the fractures are significantly wider near the wells than deep within the formation. Calculations based on injectivity/productivity data are dominated by fracture widths near the well, while calculations based on inter-well tracer data are dominated by the most restricted part of the fracture (presumably far from the wells).

Are Gel Treatments Needed? We performed a simulation study to consider whether a gel treatment would improve sweep efficiency between an injector-producer pair with a connecting fracture. We assumed (1) the pattern area was 300 by $300 \mathrm{~m}$, (2) the matrix permeability was uniformly $0.259 \mu^{2}$, and (3) a pressure drop $(\Delta p)$ of $20 \mathrm{MPa}$ was applied between the two wells. Additional details of the simulation can be found in Ref. 13. We focused on the importance of areal locations of gel plugs in reservoirs with fractures.

For a fracture width of $1 \mathrm{~mm}$, the pressure distribution is shown in Fig. 41. Because the pressure distribution was symmetrical about the fracture, only one-half of the pattern is illustrated. (The fracture is located on the front face of Fig. 41 and subsequent similar figures.) Fig. 42 shows the pressure distribution for the same half-pattern when no fracture connected the wells. For the open-fracture case, the flow rate through the half-pattern was 4.7 times greater than for the no- 
fracture case. On the positive side, the fracture allowed the pattern to experience much higher injectivity and productivity indexes than the no-fracture case. However, on the negative side, most of the injected fluid simply channeled through the fracture. Also on the positive side, higher pressure gradients were distributed more deeply through the pattern for the open-fracture case than for the no-fracture case (compare Figs. 41 and 42). For the open-fracture case, $75 \%$ of the pattern experienced a pressure gradient over $34 \mathrm{kPa} / \mathrm{m}$, while for the no-fracture case, only $26 \%$ of the pattern experienced a pressure gradient over $34 \mathrm{kPa} / \mathrm{m}$. Of course, higher pressure gradients aid in driving oil from deep within the pattern. However, from a practical view, a 20 MPa pressure difference may be difficult to maintain across the pattern when the fracture is fully open. If high flow rates overwhelm the pumps, a lower pressure drop may result - leading to lower pressure gradients throughout the pattern than indicated in Fig. 41. Incidentally, it may help to view the pressure distributions in Figs. 41-43 as "waterfalls" or inclined surfaces that direct drainage of fluid from the pattern. Near-horizontal surfaces indicate poor drainage, while steep surfaces indicate rapid drainage.

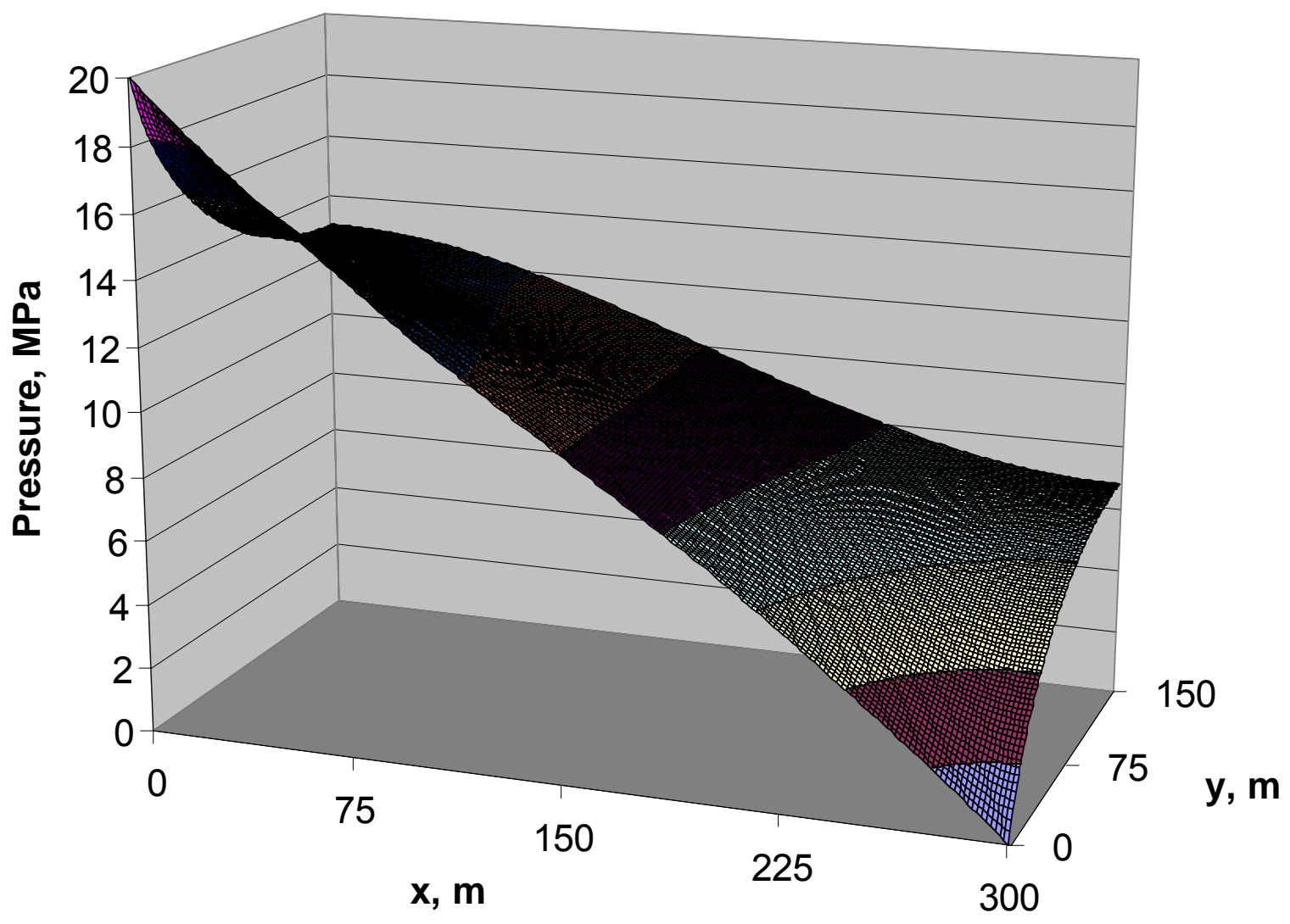

Fig. $41-$ Pressure distribution with a $1-\mathrm{mm}$ wide fracture in $0.259-\mu \mathrm{m}^{2}$ rock. 


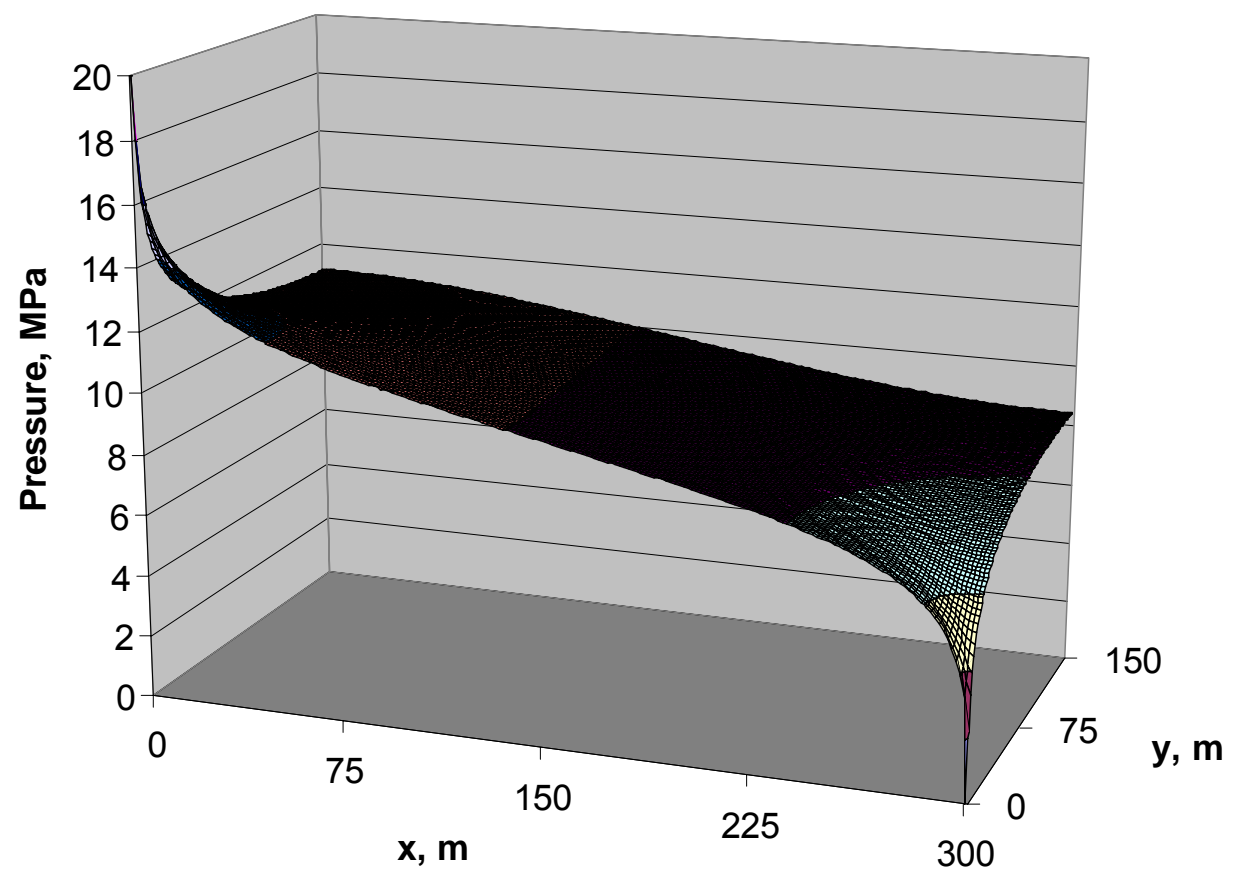

Fig. 42-Pressure distribution with no fracture present.

The greatest improvement in sweep occurs if the middle portion of the fracture can be plugged while leaving the fracture open near the wells. ${ }^{115}$ This situation allows a high fluid injectivity index, a high oil productivity index, and a fairly even sweep of most of the pattern.

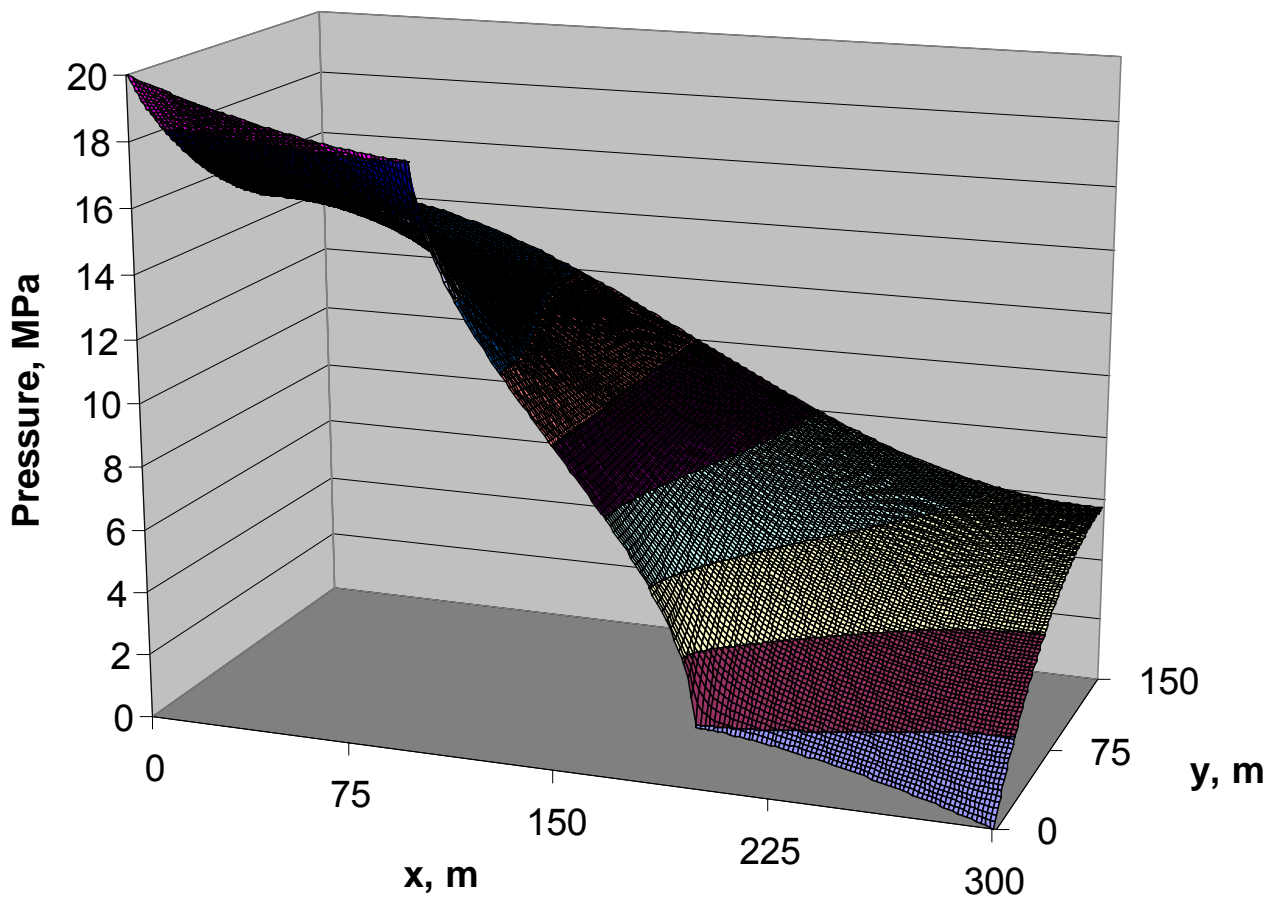

Fig. $43-w_{f}=1 \mathrm{~mm}$ for the $1 \mathrm{st}$ and $3 \mathrm{rd} 100-\mathrm{m}$ fracture sections; $w_{f}=0.15 \mathrm{~mm}$ in 2 nd section. 
For the three injector-producer pairs where we suspected channeling through fractures, the combined results from injectivity/productivity calculations and inter-well polymer breakthroughs indicated that fractures were more than $1-\mathrm{mm}$ wide near the wells, but less than $0.02-\mathrm{mm}$ wide far from the wells. Simulations were performed to examine sweep in the pattern with this and similar scenarios. Fig. 43 shows a representative result. In this case, the simulation assumed the fracture width was $1 \mathrm{~mm}$ from the injector to $100 \mathrm{~m}$ along the fracture, $0.15 \mathrm{~mm}$ for the middle $100 \mathrm{~m}$ of the fracture, and $1 \mathrm{~mm}$ for the final $100 \mathrm{~m}$ to the production well. Sweep efficiency was excellent, while injectivity and productivity indexes were high. The pressure gradient pattern shown in Fig. 43 was virtually identical to those from other simulations where the middle part of the fracture was narrower (or even when the middle fracture width was zero). The pattern in Fig. 43 was also very similar to cases where the near-wellbore parts of the fracture had widths greater than $1 \mathrm{~mm}$.

The results in Fig. 43 and from similar simulations suggest that gel treatments are not needed for the three injector-producer pairs where fractures were present. Because the fractures are narrow far from the wellbore, severe channeling does not occur. On the contrary, the existence of the fractures near the wellbore aids reservoir sweep (compare the no-fracture case in Fig. 42 with Fig. 43). Furthermore, the near-wellbore fractures substantially increase the injectivity index during injection of polymer solutions and increase oil productivity index in the production wells (i.e., by a factor of 1.8 for the cases illustrated in Figs. 42 and 43).

\section{Are “Colloidal Dispersion Gels” a Viable Option?}

"Colloidal dispersion gels" (HPAM crosslinked with aluminum citrate) were field tested at Daqing. ${ }^{116}$ Claims were made for the success of these treatments at Daqing and elsewhere. ${ }^{116-119}$ However, before committing to a more widespread application, the science and engineering of the "CDG" process should be carefully examined.

Consider how other gels perform in porous media. ${ }^{12,14,120-123}$ Early in the gelation process, most gelants behave like clean fluids that do not contain suspended particulate matter. ${ }^{12,14,120-123}$ However, after gel aggregates form and grow to the size of pore throats, gel filtration can radically increase the resistance to flow. ${ }^{120-122}$ The literature indicates that gelants can penetrate a significant distance into porous rock before gelation, but after gelation, gel propagation is extremely slow or negligible. ${ }^{12,14,120-123}$ Alternatively, if gelation is stopped sufficiently early or if gels are sufficiently sheared so that gel particles remain significantly smaller than pore throats, the gel suspensions can propagate through porous rock; however, the level of mobility reduction is generally small (less than 2). ${ }^{16}$ Also, suspensions of gel particles and adsorbed polymers provide resistance factors and residual resistance factors that increase with decreasing permeability. ${ }^{14,15,18-21}$ Not surprisingly, suspensions of small gel particles are more effective at restricting flow through small pores than through large pores. Straight-forward calculations using the Darcy equation reveal that this behavior can be detrimental for sweep improvement-both during polymer flooding and gel treatments. ${ }^{17}$

In contrast to normal gel behavior, the aluminum-citrate-HPAM gels (after gel formation) were speculated to propagate through porous rock like viscous polymer solutions. ${ }^{116-119}$ These gels were purported to act like mobility-control agents. If true, this mechanism would be radically different from that for other gels, which act exclusively as permeability-reduction agents. 
The distinction between a blocking (or permeability-reducing) agent and a mobility-control agent is an important concept to understand. A mobility-control agent should penetrate as much as possible into the less-permeable zones so that oil can be displaced from poorly swept zones. In contrast, we wish to minimize penetration of blocking agents into the less-permeable, oilproductive zones. Any blocking agent that enters the less-permeable zones can hinder subsequent injected fluids (e.g., water) from entering and displacing oil from those zones.

Laboratory Behavior of Colloidal Dispersion Gels. We performed a coreflood to test how the aluminum-citrate-HPAM colloidal dispersion gels behave. The $0.7-\mu \mathrm{m}^{2}$ Berea sandstone core had a length of $14.7 \mathrm{~cm}$ and a diameter of $3.56 \mathrm{~cm}$. An internal pressure tap was located $2.3 \mathrm{~cm}$ from the inlet sandface. A formulation was prepared that contained 300-mg/L HPAM (Tiorco $\mathrm{HiVis} 350^{\mathrm{TM}}$ ), $15-\mathrm{mg} / \mathrm{L}$ aluminum (as citrate, Tiorco $677^{\mathrm{TM}}$ ), and $0.5 \% \mathrm{KCl}$. All experiments were performed at $41^{\circ} \mathrm{C}$. After saturating the $0.7-\mu \mathrm{m}^{2}$ Berea sandstone core with brine $(0.5 \%$ $\mathrm{KCl}), 13 P V$ of polymer solution $(300-\mathrm{mg} / \mathrm{L}$ HPAM without crosslinker) were injected and the same stable residual resistance factor was observed in both sections of the core. Later, polymer solution (also 300-mg/L HPAM) with crosslinker $(15-\mathrm{mg} / \mathrm{L} \mathrm{Al}$ as citrate) was injected at the same rate (6.8 $\mathrm{PV}$ per hr). This formulation was 1 hour old at the start of gelant injection. For the first $7 P V$ of gelant injection (requiring another hour), the resistance factors in the two core sections were stable, the same, and the same as during the previous injection of polymer solution without crosslinker. Thus, for this period, the behavior of the aluminum-citrate-HPAM colloidal dispersion gel was the same as that for other gelants. Specifically, before the gelation time, the formulation behaved the same as a polymer solution without crosslinker.

Beginning at $7 \mathrm{PV}$ of gelant injection (2 hours after the gelant was prepared), the resistance factor in the first core section increased (by more than a factor of 30), while the resistance factor in the second section decreased dramatically over the course of 3 additional $P V$ of gelant injection. These results indicate that the time required for polymer crosslinking and formation of "colloidal dispersion" gel particles was only two hours at $41^{\circ} \mathrm{C}$. The gel particles were clearly filtered out by the first core section-causing the resistance factors to rise rapidly. Resistance factors in the second section dropped because the polymer and gel were stripped from the solution in the first section.

Our results confirm that the aluminum-citrate-HPAM formulation basically behaves like other gels and gelants. ${ }^{12,14,120-123}$ Early in the gelation process, it propagates through sandstone like a polymer solution without crosslinker. After some point (presumably when gel aggregates grow to the size of pore throats), gel propagation is extremely slow or negligible. Thus, aluminumcitrate-HPAM formulations should not flow through porous rock as a "super polymer" after gel formation.

The University of $\mathrm{TX}^{124}$ and the University of Kansas ${ }^{125}$ reported results consistent with our results, where the gel caused substantial permeability reduction only in the first section of a core. Both sets of researchers also extensively studied the ability of aluminum to propagate through porous rock. They found that effective propagation required high citrate/aluminum ratios and appropriate $\mathrm{pH}$ values. ${ }^{124,125}$ Aluminum precipitation and ion exchange played important roles in inhibiting aluminum propagation. 
For conditions that allowed effective propagation of aluminum, researchers ${ }^{124,126}$ found that resistance factors (effective viscosities) provided by aluminum-citrate-HPAM colloidal dispersion gels within the core (i.e., beyond the first core section) were the same as those provided by polymer with no crosslinker. This observation suggests that either the gel particles were too small to interact significantly with pore throats or the crosslinking reaction did not take place to a significant extent. These possibilities are quite consistent with the behavior for other gels. If insufficient polymer or crosslinker is present, gel formation will not take place (even small gel particles may not form). With such low aluminum concentrations (as low as $15 \mathrm{mg} / \mathrm{L}$ ), it is not surprising that a small loss of crosslinker (e.g., by ion exchange or precipitation) could preclude formation of adequate gel particles. (Formation of conventional gels was reported using aluminum concentrations of at least $200 \mathrm{mg} / \mathrm{L}$, provided the ratio of citrate to aluminum was in the proper range. ${ }^{127,128}$ )

Requirements for a Viable Colloidal Dispersion Gel. If one wished to develop and demonstrate positive behavior for a new suspension of gel particles, a number of useful and informative experiments could be performed. First, experiments must be performed using cores with multiple sections (e.g., multiple internal pressure taps). The injected formulation (1) must propagate into the rock without causing progressive plugging of the inlet sand face, (2) must show uniform resistance factors and residual resistance factors along the various core sections, (3) must propagate these resistance factors through the porous rock at an acceptable rate (i.e., no excessive chemical retention), AND (4) must provide greater resistance factors/residual resistance factors than polymer solution alone. One must also be concerned about the magnitude of residual resistance factors as a function of rock permeability. ${ }^{14,15,18-21}$ This latter comparison must be made using cores that were completely filled with polymer or gel (i.e., NOT using misleading parallel corefloods). If residual resistance factors in low permeability rock are significantly greater than in high permeability rock, polymer or gel treatments can impair sweep efficiency. $^{13,17}$

Parallel corefloods, especially parallel linear corefloods, should NOT be used to evaluate the diversion properties of gels. Parallel corefloods are easily manipulated to give the appearance of successful fluid diversion. ${ }^{15}$ Test results from parallel linear corefloods, in particular, cannot be directly translated to profile modification in radial flow (i.e., unfractured wells). ${ }^{13,17}$ Also, with short laboratory cores, diffusion and dispersion can readily compromise small gelant banks in the less permeable of the parallel cores-giving the false impression that gelant does not significantly enter or damage less permeable oil zones. ${ }^{129}$ In real field applications, the distance of gelant penetration is several feet or more, even in the least permeable oil-productive zones. ${ }^{13,17,112}$ For these distances, diffusion and dispersion will not destroy gelant banks. ${ }^{129}$

Consideration of Field Data. In general, the field results for applications of colloidal dispersion gels fit into one of several categories. ${ }^{13}$ In the first category, the treated wells contained no fractures or linear flow features. For these cases, the aluminum was probably either removed (by adsorption on rock) or was present in concentrations too low to allow crosslinking-so the polymer could propagate through the formation and provide some benefit as a polymer flood. This scenario is very consistent with the laboratory findings from the University of $\mathrm{TX}^{124}$ and the University of Kansas. ${ }^{126}$ Since the aluminum provided no benefit for this scenario, the money spent on aluminum citrate would have been more effectively spent on more polymer. 
In the second field category, the treated wells probably contained fractures or fracture-like features (even though the operator or gel vendor may not have been aware of these before the treatments). For these cases, the gels may have provided some benefit from partially plugging the fractures. However, for moderate to wide fractures, other types of gels [e.g., Cr(III)-acetateHPAM) probably would have been more effective]. On a positive note for narrow fractures, colloidal dispersion gels conceivably may be more effective than other gels because of more effective penetration into tight fractures. ${ }^{130,131}$ This possibility requires further investigation.

In the third field category, the treated wells experienced general plugging of all open zones and flow paths. From an areal view, these wells were key to water channeling. By reducing the flow capacity of the treated wells, areal pressure gradients were altered so water injected into other (non-treated) wells pushed incremental oil toward offset production wells. This benefit could be realized by any means that reduced the flow capacity of the treated wells. Well flow capacity can usually be reduced more cost-effectively using other gels and methods.

In the fourth field category, the reported benefits and/or increases in oil recovery had nothing to do with the colloidal dispersion gels. In some cases, the reported benefits for particular wells and fields occurred because of other changes or improvements that were implemented. In other cases, no real benefit occurred. The reported benefit resulted from an overly pessimistic projection of the pre-treatment decline curve and/or an overly optimistic assignment of incremental oil to the gel treatment.

Careful analysis of the Daqing field data presented in Ref. 116 and additional unpublished data associated with the project indicates no significant difference between the "CDG" flood and the normal polymer flood. Injectivity behavior was not significantly different for the two cases. (Injectivity was actually slightly higher during CDG injection.) Also, water/oil ratios and production trends cannot be credibly distinguished for the two processes. These results are consistent with the first category of field results mentioned above: the aluminum was either removed (by adsorption or precipitation) or was present in concentrations too low to allow crosslinking.

\section{Mitigating Polymer Degradation}

If channeling truly occurred during the applied polymer floods, it is possible that polymer degradation was responsible for sweep efficiency being lower than expected. This section considers causes of polymer degradation and their mitigation.

Mechanical or Shear Degradation. During preparation and injection, the polymer solution passed through a number of mixers, pumps, valves, filters, and pipes. Flow through this equipment can break polymer chains and decrease solution viscosity to some extent (called shear or mechanical degradation). Fig. 44 shows viscosity data of samples at different points along the polymer flow stream. Most viscosity loss occurred from the high pressure injection pumps and mixing system to the near-wellbore - amounting to about $64 \%$ of the total loss. Consistent with other work, ${ }^{132}$ the greatest restriction to flow and the greatest mechanical degradation occurred from entering the porous rock at the high velocities at the injection sand face. 


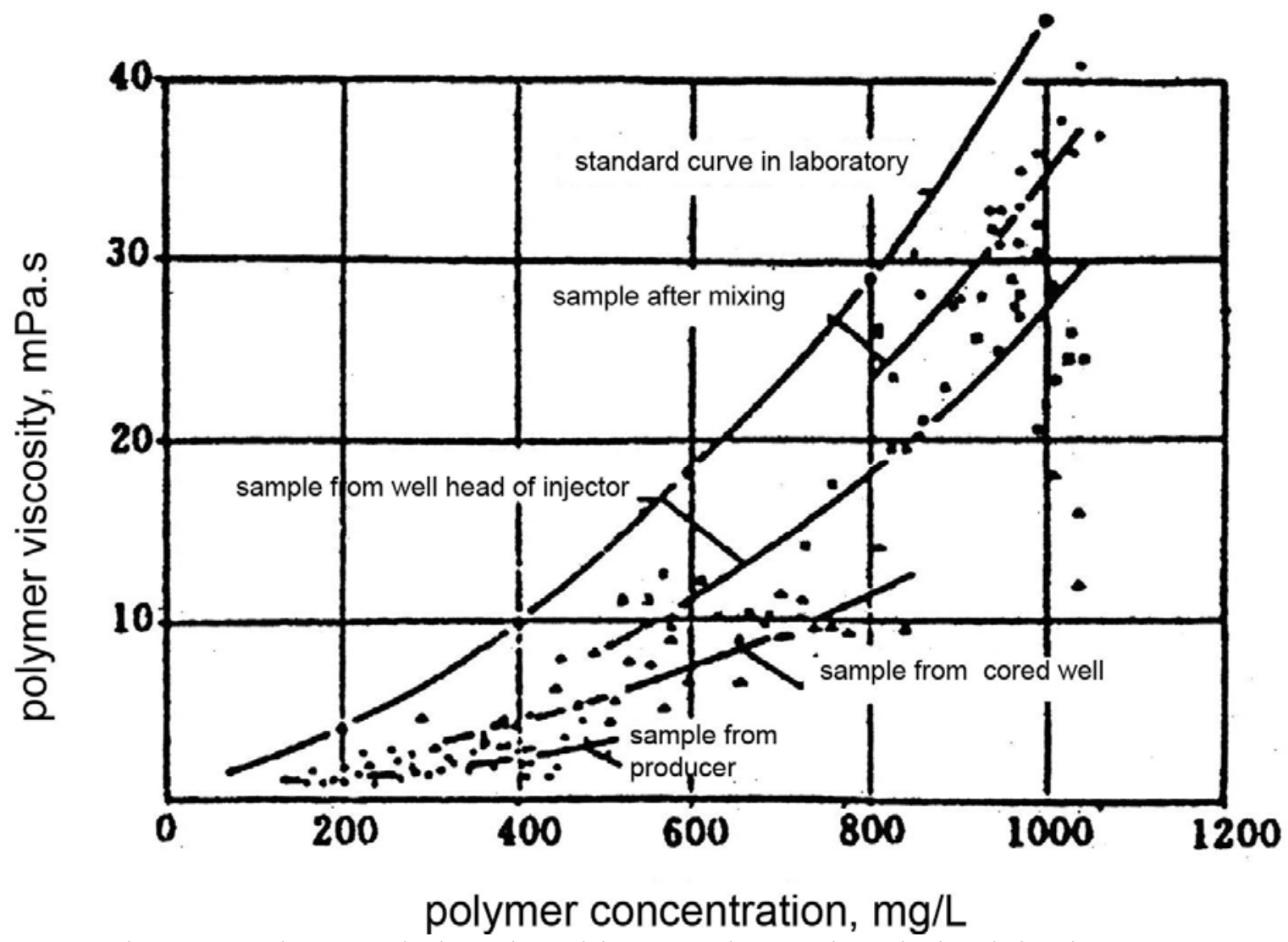

Fig. 44-Polymer solution viscosities at various points during injection.

The presence of a fracture in a well can mitigate mechanical degradation. ${ }^{133}$ Fractures provide a dramatic increase in flow area at the injection sand face, thus decreasing the velocity of the polymer solution at this important constriction, and thereby reducing mechanical degradation. Also, the presence of fractures in wells can significantly increase well injectivity and productivity indexes. However, if fractures are used for these purposes, care must be taken so that the orientation and length of the fractures are not such that they accentuate channeling. ${ }^{134,135}$ The analysis presented earlier indicates that fractures in the target Daqing wells satisfy this requirement. Consequently, there may be considerable value in intentionally fracturing (and perhaps propping) injection wells during future chemical flooding projects at Daqing.

Oxidative Degradation. Fresh water was used to prepare polymer solutions in the Daqing field projects. This water had a high concentration of dissolved oxygen, and no processing was used to remove it. The presence of dissolved oxygen, free radicals, and/or a redox couple can severely degrade HPAM polymers. ${ }^{136,137}$ Thus, oxidative degradation could be a serious concern. Three observations help to mitigate this concern. First, the reservoir temperature is relatively low $\left(45^{\circ} \mathrm{C}\right)$, which should inhibit free radical generation. Second, oil in the reservoir should combine with oxygen and (hopefully) eliminate it. Third (and most convincingly), polymer produced from the reservoir was not reduced in molecular weight by more than $50 \%$ compared to the injected polymer. If oxidative degradation was important, the polymer should have been degraded to a low molecular weight, and polymer solution viscosity should have been reduced to that near 
water. ${ }^{136,137}$ Thus, mechanical degradation (as discussed above) seems a more serious issue for Daqing than oxidative degradation. If future efforts are made to reduce dissolved oxygen in the water, gas stripping is probably a more technically and economically effective method than use of chemical oxygen scavengers.

Microbial Degradation. In concept, microbial attack could also lead to polymer degradation and viscosity loss. However, two factors indicated that microbial degradation was not responsible for the viscosity losses noted at Daqing to date. First, although microorganisms have been known to metabolize the side groups on HPAM and to flocculate with the polymer, degradation of the HPAM backbone has not been proven. Second, evidence of extensive microbial activity at Daqing has not been reported.

Of course, biocides can be used to mitigate concern about microbial degradation. A successful test was performed using a biocide in Shuanhe in the Henan oil field, where the reservoir temperature was $72^{\circ} \mathrm{C}$.

\section{More Viscous Polymer Solutions}

Using Higher Polymer Concentrations. Mobility ratios and reservoir sweep can be improved by injecting more viscous polymer solutions - i.e., solutions with higher polymer concentrations. This basic concept from accepted reservoir engineering concepts can readily be confirmed using numerical simulation. Given the same volume of injected polymer solution, higher polymer concentrations yield more oil production (OOIP). An added benefit is that the higher polymer concentrations are more tolerant to mechanical degradation. ${ }^{138}$ Furthermore, they impart greater solution viscoelasticity, which provides higher oil recoveries and lower residual oil saturations under Daqing conditions. ${ }^{139}$

From the two pilot tests ${ }^{140}$ at Daqing (specifically, at areas called "West of Center" and "4-4\# Station of the Northwest in Lamadian"), the following observations were noted: (1) Water intake profile was improved after injecting higher polymer concentrations. (2) During polymer flooding, the period of relatively low water cuts can be prolonged by injecting higher polymer concentrations. (3) Interestingly, injection of more concentrated polymer solutions $(2,000-2,500$ $\mathrm{mg} / \mathrm{L}$ rather than $1,000 \mathrm{mg} / \mathrm{L})$ did not result in substantially increased injection pressures. ${ }^{140}$ This result suggests that fractures exist near the wells. If these fractures are extended during injection of the more viscous polymer solutions, increases in injection pressure will be mitigated. Both tests involving higher polymer concentrations were implemented in the later stages of the main polymer flood. In both cases, the tests were begun after the main response to the polymer flood had occurred and water cut had resumed its rise. (As mentioned above, injection of the more concentrated polymer alleviated this rise in water cut.) These tests are still underway, so the economic viability of this process change must await further results, and be evaluated in light of the chemical costs, oil price, and geographic factors associated with the test site. Another pilot test was initiated in May 2005 in a Daqing pattern that was not previously polymer flooded (1\# of East Area of the First Area of South). This test may provide the most definitive evaluation of the high-polymer-concentration process. As of April 2006, about $0.1 \mathrm{PV}$ of polymer solution has been injected. Injection pressures are only $1 \mathrm{MPa}$ greater than an adjacent area where polymer solution with the original (lower) concentration was injected. Current injection pressures are 
thought to be below the fracture pressure (3 to $4 \mathrm{MPa}$ ). The project will continue to be monitored.

New Salinity-Tolerant Polymer. A new salinity-tolerant polymer ${ }^{141,142}$ (called "KYPAM") was developed at Daqing that provides higher viscosities in saline waters than those provided by conventional HPAM polymers. This new copolymer incorporates a small fraction of new monomers with acrylamide to form comb-like copolymers. There are currently two varieties of this "KYPAM" polymer. One has a high molecular weight (20-25 million daltons) and uses as the functional group-AHPE (aromatic hydrocarbon with ethylene). At present, we are most interested in the second variety, which has medium (12-17 million daltons) or low (3-11 million daltons) molecular weight. The medium molecular weight copolymer incorporates the monomer, 2-mercaptobenzimidazole, while the low molecular weight copolymer contains RSO (sulfhydryl).

Table 11 compares viscosities for the new polymer with conventional HPAM polymers-one with ultra-high molecular weight (35 million daltons) and one with low-to-medium molecular weight (7 15 million daltons). Viscosities were measured in waters with two salinities $-1,000$ $\mathrm{mg} / \mathrm{L}$ and 4,000 $\mathrm{mg} / \mathrm{L}$. The salinity-tolerant polymer provided the highest viscosity in the more saline water, and it experienced a smaller percentage change in viscosity in the two waters.

Table $11-$ Viscosities for solutions with $1,000 \mathrm{mg} / \mathrm{L}$ polymer, $45^{\circ} \mathrm{C}$

\begin{tabular}{|c|c|c|c|}
\hline Salinity (mg/L) & 1,000 & 4,000 & Ratio, \% \\
\hline Ultra-high Mw HPAM & $82.8 \mathrm{mPa}-\mathrm{s}$ & $40.3 \mathrm{mPa}-\mathrm{s}$ & 48.7 \\
\hline $\begin{array}{c}\text { Salinity-tolerant polymer } \\
\text { With medium Mw }\end{array}$ & $62.2 \mathrm{mPa}-\mathrm{s}$ & $52.3 \mathrm{mPa}-\mathrm{s}$ & 84.1 \\
\hline Medium Mw HPAM & $38.9 \mathrm{mPa}-\mathrm{s}$ & $16.8 \mathrm{mPa}-\mathrm{s}$ & 43.2 \\
\hline
\end{tabular}

The above polymers can all be produced at about the same cost, so polymer performance is the main criterion of importance. In more saline waters, the salinity-tolerant polymer will be preferred. For very fresh waters and high-permeability rock, the ultra-high Mw polymer will be preferred. In very fresh water and less-permeable rock, the medium Mw polymer may be most functional.

Using Other New Polymers. A new water-soluble hydrophobic associative polymer (called APP4) was tested in Zhongyuan Oil Field in Central China. Based on preliminary results from the pilot, Well W94-4 produced 4.5 tons/day of incremental oil. Water cut declined 4\%.

This polymer can be dissolved as rapidly as HPAM - for example, a solution with 5,000 mg/L can be prepared in 2 hours if the temperature is greater than $35^{\circ} \mathrm{C} .{ }^{143}$ Compared to the KYPAM polymer, AP-P4 can provide greater viscosity.

At present, the cost of this new hydrophobic associative polymer is $10 \%$ more than HPAM polymers, but it can provide a larger financial internal rate of return. Further laboratory testing is underway to determine if the new hydrophobic associative polymer will provide superior performance to HPAM in the important areas of polymer stability and retention. 
Recently, displacement experiments were performed comparing xanthan and HPAM. ${ }^{140}$ For either a given polymer concentration or viscosity level, HPAM solutions provided significantly greater oil recovery levels under Daqing conditions. This result was believed to occur because HPAM solutions exhibited significantly greater viscoelasticity than xanthan solutions.

Use of Less Saline Water. HPAM and other anionic polymers are well known to provide much higher viscosities in fresh water than in saline waters. Maitin ${ }^{144}$ demonstrated that low salinity HPAM solutions can sweep effectively through high salinity reservoirs (i.e., without destructive mixing and salinity increase in the polymer bank) if mobility control is maintained. At Daqing, the lowest water salinity used for polymer mixing contained about $300 \mathrm{mg} / \mathrm{L}$ total dissolved solids (TDS). However, significant variations in water salinity occur. Water quality varies with the amount of rain, surface temperature, and humidity during the four seasons. Also, the content of $\mathrm{Ca}^{2+}$ and $\mathrm{Mg}^{2+}$ in the Daqing water is lower in summer and higher in winter.

Can reverse osmosis be practically applied to prepare polymer solutions with less than $200 \mathrm{mg} / \mathrm{L}$ TDS at Daqing? This question remains to be investigated. At present, use of available fresh water ( $\sim 400 \mathrm{mg} / \mathrm{L}$ TDS) for polymer mixing costs $\$ 0.49 / \mathrm{m}^{3}$ more than use of produced water $(\sim 3,000$ $\mathrm{mg} / \mathrm{L}$ TDS). Current thinking at Daqing is directed more toward development of polymers that provide the desired viscosities and resistance factors using Daqing produced water.

\section{Are Foams a Viable Option?}

Limiting Capillary Pressure. In concept, foams are low-mobility gas/water/surfactant formulations that could replace polymer solutions for mobility control. ${ }^{145,146}$ In addition to the sweep benefits from injecting a low-mobility fluid, the limiting capillary pressure phenomenon may provide an additional advantage: formation of a low-mobility foam in high-permeability zones while a non-foaming, high-mobility gas/water composition flows freely through lowpermeability zones. Foam flow generally occurs in one of two regimes. ${ }^{145}$ In one regime, foam quality is low (low gas fraction), and gas mobility is quite low. However, liquid velocity is quite dependent on pressure gradient. In the second regime, limiting capillary pressure is effective in collapsing the foam, foam "quality" is high (high gas fraction), and gas velocity is insensitive to pressure gradient over a wide range.

To briefly explain the limiting capillary pressure concept, consider two gas bubbles that are flowing through a porous medium. Because of their close proximity, these bubbles are separated by a film of water. A pressure difference, called the capillary pressure, exists between the gas phase and the liquid phase. The limiting-capillary-pressure concept recognizes that if the capillary pressure is too great, water will be sucked away from the film, the film separating the bubbles will collapse, and the bubbles will coalesce. The capillary pressure at which this coalescence occurs is called the limiting capillary pressure. Greater hydrophilic rock surface area associated with less-permeable rock promotes foam collapse in low-permeability rock. ${ }^{147}$

Although foams have significant potential, they have notable deficiencies and disadvantages. First, propagation through a reservoir rock can be severely limited by surfactant retention and partitioning into oil. Second, costs to compress and inject gas or foam can be quite high. Third, involved laboratory tests may be needed to identify conditions that allow effective foam generation and especially exploitation of the limiting-capillary-pressure effect. 
ASP Foam Flooding. ASP Foam (ASPF) flooding was developed as a new EOR technology, combining foam flooding and ASP flooding. Consisting of alkali, surfactant, polymer and gas, the ASPF system not only can greatly reduce the interfacial tension (IFT) between the crude and the complex system, and thus increase the displacement efficiency, but also can reduce the mobility ratio to enlarge the swept volume. Therefore, ASPF flooding could enhance oil recovery more than ASP flooding. A successful field test of the first ultra-low interfacial tension foam flood was performed at Daqing. ${ }^{148}$ This project increased volumetric sweep efficiency, and the final recovery should be around $70 \%$ OOIP.

Foam Stability in ASPF Floods. Surfactant, polymer, and alkali all impact foam stability. Of course, foam generation and stability are strongly influenced by the type and concentration of surfactant. ${ }^{149}$ Surfactant bilayers are essential to generate and stabilize the thin liquid films that separate the gas bubbles that comprise the foam. The surfactants are also needed to provide ultralow interfacial tension in the ASP process. Thus, either one surfactant must fulfill dual functions, or if two or more surfactants are used, their actions must not have a negative interference with the function of the other surfactant.

Polymer concentration and molecular weight are also well known as factors that impact foam stability. ${ }^{150}$ Foam stability is directly related to the rate of liquid drainage from films, which in turn is directly related to liquid viscosity-which is, of course, impacted by polymer concentration and molecular weight.

Since salinity impacts surfactant behavior and foam stability ${ }^{149,151}$ and since salinity is directly related to alkali concentration, foam stability is affected by the alkali. Increased salinity acts to screen charged surfactants in solution, thereby affecting surfactant aggregation (e.g., critical micelle formation and film formation). ${ }^{149,151}$ Alkali can neutralize organic acids in oils, generating additional surfactants that may act in either a positive or negative manner with existing surfactants that are intended to generate foams. ${ }^{152}$

During the Daqing field test of the first ultra-low interfacial tension foam flooding, ${ }^{148}$ a relatively stable foam slug was formed in the formation. Injection pressures during this ASPF pilot were higher than when injecting water, HPAM, or ASP formulations. The gas/oil ratio (GOR) after this pilot was significantly lower than when this same area was on WAG flooding. This result also supports that foam was formed in the formation and prevented gas fingering and channeling. ${ }^{148}$

\section{Thermal Recovery or MEOR}

For the ultra-low permeable areas in Daqing Oil Field, thermal recovery methods (i.e., steam cycling and steam drive) and "Bio-Huff-Puff " are also methods that are under consideration for sweep improvement.

A "Bio-Huff-Puff" pilot test was successfully conducted in 47 wells in the Chaoyanggou area of Daqing in 2002. The Bio-Huff-Puff process uses microorganisms to improve injection profiles. The 47-well test achieved a positive response in the main oil zones for $80 \%$ of the treated wells. Based on this successful pilot, the test scale was enlarged in 2003. Laboratory results indicate that $7.2 \%$ OOIP EOR can be achieved in some cases using microbial profile modification methods after polymer flooding. 
A field test of steam cycling was successful in ultra-low permeable oil zones in another area of Chaoyanggou - proving that this can be another choice for sweep improvement.

\section{Pattern Spacing}

Consideration of the effects of pattern spacing on sweep efficiency during polymer flooding is included in Ref. 153.

\section{Conclusions}

Sweep improvement in the Daqing Oil Field was considered using various approaches. Given that the reservoir description and polymer input data were correct, the polymer flood should have provided excellent sweep throughout the majority of the Daqing patterns under consideration. If ASP flooding is applied to increase recovery efficiency, mobility control and sweep improvement will be especially important and challenging during implementation.

In a number of Daqing wells (both injectors and producers), fractures were present. These fractures were narrow far from the wellbore, so channeling was not significant. On the contrary, these near-wellbore fractures aided reservoir sweep. They substantially increased the injectivity index during injection of polymer solutions and increased oil productivity index in the production wells. Near-wellbore fractures with the proper length, orientation, and width may play a crucial role during future floods if very viscous chemical (i.e., ASP) banks must be injected to maintain mobility control. Appropriate use of near-wellbore fractures may also mitigate mechanical degradation.

Cost-effective improvements at Daqing may be realized using one or more new polymers. ASP foams, steam, and microbes showed positive indications during field tests. Analyses of laboratory and field results raise doubts about the viability of colloidal dispersion gels. Additional work may be warranted concerning the potential of foams and polymer solutions prepared with reduced salinity. 


\section{ARE COLLOIDAL DISPERSION GELS REALLY A VIABLE TECHNOLOGY?}

\section{The Controversy}

February 2006 JPT. In the February 2006 issue of the Journal of Petroleum Technology (JPT) H.L. Chang et al. ${ }^{154}$ summarized some of the pilot and commercial-scale field activities on polymer flooding and ASP flooding that were performed in China. Unquestionably, polymer flooding and ASP flooding can be effective oil-recovery processes and have great potential. Unfortunately, the paper also advocated a controversial technology (flooding with aqueous colloidal dispersion gels) as being superior to polymer flooding. This claim is misleading and generally incorrect. "Colloidal dispersion gels" ("CDG" or relatively low concentrations of HPAM crosslinked with aluminum citrate) should not be applied without carefully examining the purported science and engineering behind this process.

Chang and the CDG vendor that he represents speculated that low-concentration aluminumcitrate-HPAM micro gels propagate through porous rock like super polymer solutions. ${ }^{116-118,154}$ Specifically, they suggested that these CDG formulations penetrate deep into porous matrix reservoir rock and subsequently provide higher resistance factors (effective viscosities in porous media) and residual resistance factors (permeability reduction factors) than comparable HPAM polymer solutions without crosslinker.

Because Chang's paper was published in the "SPE Distinguished Author Series," the Society of Petroleum Engineers may be perceived as endorsing colloidal dispersion gels as an accepted, state-of-the-art, better-than-polymer-flooding technology. The purpose of this chapter is to question that status.

Controversy Background. To understand this controversy, the reader must first recognize the distinction between a conformance treatment (i.e., permeability-reduction or blocking agent) and a polymer flood (mobility-control agent). ${ }^{13}$ Conventional gels used in "conformance control" are intended to block or reduce the flow capacity of high-permeability channels without damaging less-permeable hydrocarbon-productive zones (Fig. 45). In this situation, the objective is to minimize penetration of gelants or permeability-reducing agents into the less-permeable, oilproductive zones. Any gel or blocking agent that enters the less-permeable zones can hinder (or even shut off) subsequent injected fluids (e.g., water) from entering and displacing oil from those zones. In contrast, polymer floods and similar mobility-control methods are intended to directly displace oil from less-permeable zones (as well as improve mobility ratio and sweep in any given zone.) Consequently, a polymer solution should penetrate as much as possible into the lesspermeable zones so that oil can be displaced from these poorly swept zones. For any material that enters the hydrocarbon zones, the engineer must ask, Will this damage the flow capacity of my hydrocarbon zone more than that of the water zone? 


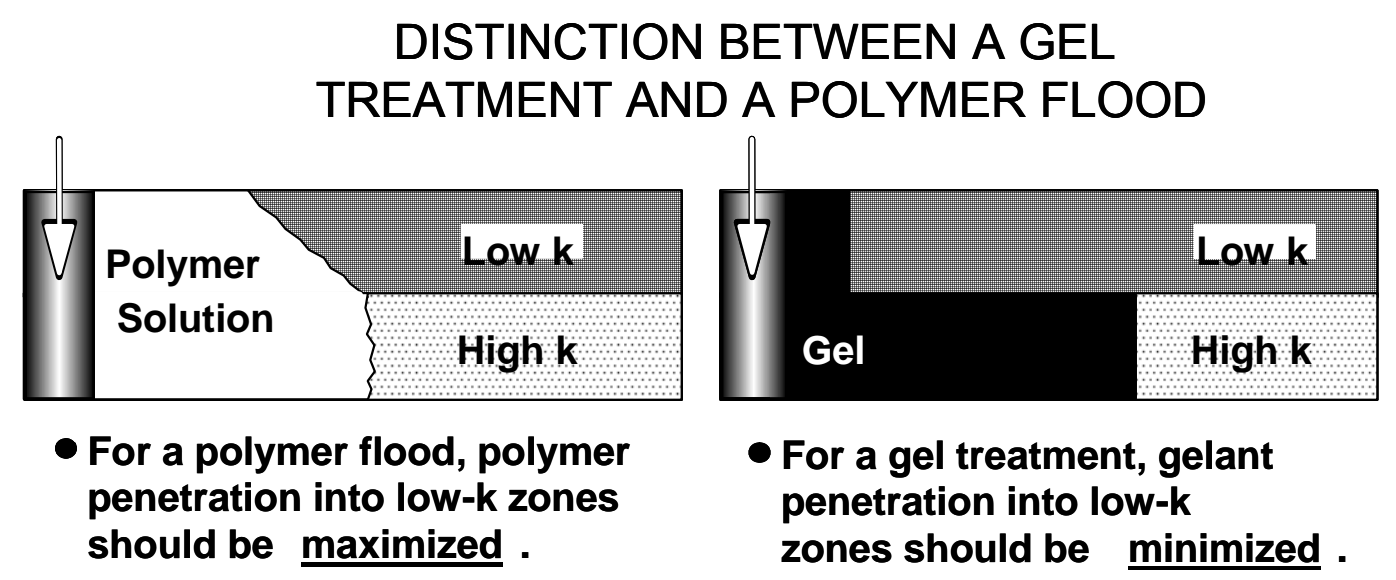

Fig. 45-Distinction between a gel treatment and a polymer flood.

Polymers and Gelants Can Flow Through Rock. Gels Do Not. Consider how crosslinked polymer gels perform in porous media during conformance-improvement treatments. . $^{12,14,120-123}$ Early in the gelation process, most gelants behave like clean fluids that do not contain suspended particulate matter. ${ }^{12,14,120-123}$ However, after the first gel aggregates form and grow to the size of pore throats, filtration of the gel aggregates (within the porous rock) can radically increase the resistance to flow. ${ }^{120-122}$ Gelants (e.g., polymer-crosslinker solutions prior to significant polymer crosslinking) can penetrate a significant distance into porous rock before gelation, but after gelation, gel propagation is extremely slow or non existent. ${ }^{12,14,120-123}$ The gelation onset time of aluminum-citrate-HPAM CDG formulations are relatively short (a few hours at $40-50^{\circ} \mathrm{C}$ ). If gelation is stopped sufficiently early or if gels are sufficiently sheared so that gel particles remain significantly smaller than pore throats, the gel suspensions can propagate through porous rock; however, the level of mobility reduction (residual resistance factor) is generally small (less than 2). ${ }^{16}$ Independent studies at several locations (the University of Kansas, ${ }^{125,126,155}$ the University of TX, ${ }^{124}$ New Mexico Tech, ${ }^{156,157}$ Stavanger College, ${ }^{128}$ and $\mathrm{BP}^{127}$ ) confirm that aluminum-citrateHPAM gelants and gels behave like other gelants and gels.

Chang's Claim Violates Darcy's Law. In Ref. 154, Chang claimed that "a large amount of CDG would preferentially enter the high-permeability or thief zones and divert polymer or water into medium- and low-permeability zones." This unsupported assertion contradicts basic calculations using Darcy's Law, as demonstrated by Refs. 13, 17, and 129. To make these calculations readily accessible, the reader can download and use the first spreadsheet from our web site at: http://baervan.nmt.edu/randy/designing\%20gel\%20treatments/Topics.htm. By experimenting with this spreadsheet, the reader should appreciate that the polymer or gelant DOES NOT "preferentially enter the high-permeability or thief zones." Instead, it penetrates into each zone in accordance with Darcy's Law. In fact, for a given volume of fluid injection, viscous solutions penetrate proportionately farther into low-permeability zones than water. That is a basic principle of polymer flooding and fluid displacement that has been known for many years. Table 12 gives an example to illustrate this point when no crossflow occurs between layers. If crossflow can occur, the distances of gelant penetration into the less-permeable zones (relative to that in the most-permeable zone) will be significantly greater. This point can be appreciated by viewing the videos at $\mathrm{http}$ ///baervan.nmt.edu/randy/Video.htm. 
Table 12-Viscous solutions penetrate proportionately more into low-k zones.

$100,000 \mathrm{bbl}$ gelant injected. All zones have $h=10 \mathrm{ft}, \phi=0.25, S_{o r}=0.3$.

Inaccessible $P V=0.2$. Polymer retention $=1 P V / P V$.

\begin{tabular}{|c|c|c|c|c|}
\hline & & \multicolumn{3}{|c|}{ Gelant radius, ft } \\
\hline Layer & $k$, md & 1-cp gelant & 10 -cp gelant & 40 -cp gelant \\
\hline 1 & 1,000 & 173.9 & 170.9 & 170.0 \\
\hline 2 & 500 & 123.0 & 123.6 & 123.7 \\
\hline 3 & 250 & 87.0 & 89.5 & 90.2 \\
\hline 4 & 125 & 61.5 & 64.5 & 65.9 \\
\hline
\end{tabular}

Polymers and Gels Plug Low-k Rock More than High-k Rock. Will the flow profile be improved if water is injected after the polymer or gel has been placed? Chang claims that after placement, the CDG gel will "divert water into medium- and low-permeability zones." For radial flow, this claim is untrue. Suspensions of gel particles and adsorbed polymers [and adsorbed gel aggregates] provide resistance factors and residual resistance factors that increase with decreasing absolute permeability of the treated matrix reservoir rock. ${ }^{14,15,18}$ (Fig. 46 illustrates this fact for a common HPAM solution.) Not surprisingly, polymers and suspensions of small gel particles are more effective at restricting flow through small pore throats than through large pore throats. That is, the polymers or gel particles reduce the permeability of low-permeability reservoir matrix rock more than they reduce the permeability of the problematic highpermeability reservoir rock - the opposite of what is desired. Straightforward calculations using the Darcy equation reveal that this behavior is detrimental for sweep improvement - both during polymer flooding and gel treatments. ${ }^{13,17,129}$

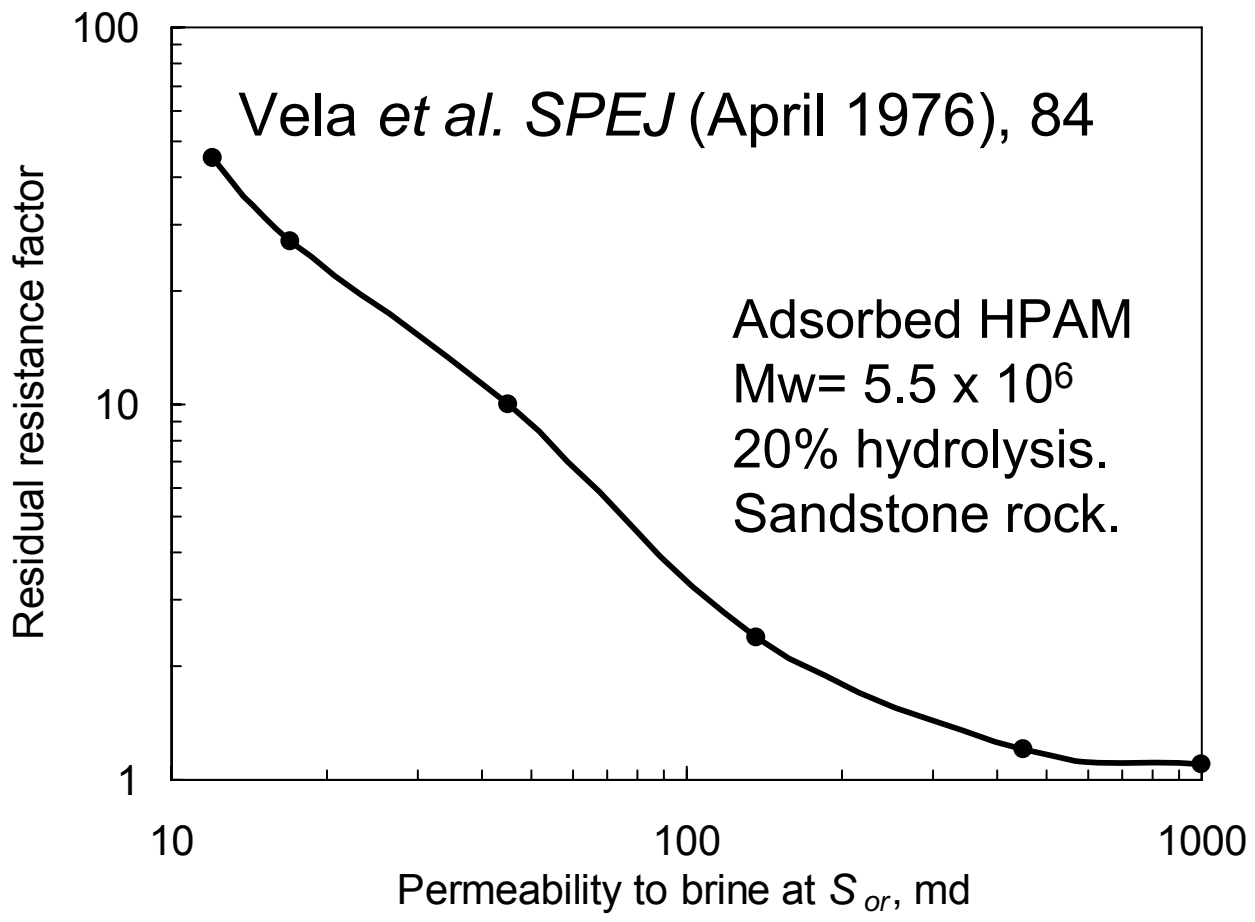

Fig. $46-F_{r r}$ versus $k$ for an HPAM solution. 
To appreciate this fact, the reader can again use the first spreadsheet on the website at http://baervan.nmt.edu/randy/designing\%20gel\%20treatments/Topics.htm. From Table 12, we used placement numbers (i.e., gelant radii) associated with the 10-cp polymer or gelant. Three cases were considered (Table 13). In each case, water was injected after polymer or gelant placement (again no crossflow), and for each of the four zones, we calculated the water injectivity index after polymer/gel placement relative to the value before placement $\left(I / I_{o}\right)$. Case 1, the most optimistic case, assumed that the residual resistance factors $\left(F_{r r}\right)$ were the same in all layers. For this case, the polymer treatment did reduce the flow capacity of Layer 1 slightly more than that of Layer 4 (0.86 versus 0.88$)$. However, the improvement was so slight that it would not be considered of practical significance in most applications. Further, existing knowledge (e.g., Fig. 46) lends no support that residual resistance factors would be the same in all layers.

Table 13-Individual zone flow capacities: after/before gel placement $\left(I / I_{o}\right)$.

$100,000 \mathrm{bbl}$ gelant injected. All zones have $h=10 \mathrm{ft}, \phi=0.25, S_{o r}=0.3$. Inaccessible $P V=0.2$. Polymer retention $=1 P V / P V$.

\begin{tabular}{|c|c|c|c|c|c|c|c|c|}
\hline & & & \multicolumn{2}{|c|}{ Case 1 } & \multicolumn{2}{c|}{ Case 2 } & \multicolumn{2}{c|}{ Case 3 } \\
\hline Layer & $k$, md & Gelant radius, $\mathrm{ft}$ & $F_{r r}$ & $\boldsymbol{I}_{\boldsymbol{o}}$ & $F_{r r}$ & $\boldsymbol{I}_{\boldsymbol{o}}$ & $F_{r r}$ & $\boldsymbol{I} / \boldsymbol{I}_{\boldsymbol{o}}$ \\
\hline 1 & 1,000 & 170.9 & 1.5 & $\mathbf{0 . 8 6}$ & 1.1 & $\mathbf{0 . 9 7}$ & 1.5 & $\mathbf{0 . 8 6}$ \\
\hline 2 & 500 & 123.6 & 1.5 & $\mathbf{0 . 8 6}$ & 1.2 & $\mathbf{0 . 9 4}$ & 2 & $\mathbf{0 . 6 8}$ \\
\hline 3 & 250 & 89.5 & 1.5 & $\mathbf{0 . 8 7}$ & 1.8 & $\mathbf{0 . 8 2}$ & 2.5 & $\mathbf{0 . 6 3}$ \\
\hline 4 & 125 & 64.5 & 1.5 & $\mathbf{0 . 8 8}$ & 2.3 & $\mathbf{0 . 7 5}$ & 3 & $\mathbf{0 . 5 9}$ \\
\hline
\end{tabular}

For Case 2, data was taken from the literature (Ref. 24), representing measured residual resistance factors for a polyacrylamide (HPAM) solution as a function of permeability (Fig. 46). Here, the residual resistance factor increased from 1.1 to 2.3 as layer permeability decreased from 1,000 to $125 \mathrm{md}$. The flow profile was noticeably harmed by the HPAM solution-because $I / I_{o}$ decreased from 0.97 to 0.75 as permeability decreased from 1,000 to $125 \mathrm{md}$.

For Case 3, the residual resistance factor increased moderately with decreasing permeability (from 1.5 to 3 as layer permeability decreased from 1,000 to $125 \mathrm{md}$ ). In this case, which might be representative of the behavior of a CDG gel, the flow profile was significantly harmed by the polymer/gel treatment - because $I / I_{o}$ decreased from 0.86 to 0.59 as permeability decreased from 1,000 to $125 \mathrm{md}$. Thus, if the CDG claim of Ref. 154 was true (that CDG gels provide higher resistance factors and residual resistance factors than polymer alone), CDG gels could actually harm sweep efficiency.

In summary, if credible choices are made for the residual resistance factors (factors by which polymer or gel reduces permeability in the polymer- or gel-contacted rock), no significant improvement in sweep will result, beyond the benefit achieved with a normal polymer flood. During water injection after the polymer or CDG flood, sweep efficiency will not generally be better than before the polymer flood. If CDG gels truly provide greater residual resistance factors than uncrosslinked polymer, then $\mathrm{CDG}$ residual resistance factors should increase with decreasing permeability in a way that is more extreme than for polymer alone. Consequently, CDG gels can actually HARM sweep efficiency, when compared to a normal polymer flood. The best that can be reasonably hoped for is that the aluminum citrate does nothing-i.e., has no effect on the polymer. In that case, why waste money on the aluminum citrate? 


\section{Laboratory Results}

Many Previous Lab Tests Show No Effect of Aluminum on Polymer. Independent tests from several university and industry laboratories confirm that aluminum-citrate-HPAM "colloidal dispersion" gelants and gels show the same basic traits as other gels used for conformance improvement. $^{124-128,155,156}$ That is, they are NOT super-polymer flooding agents. In particular, researchers from the University of Kansas ${ }^{125,126}$, the University of $\mathrm{TX}^{124}$, and New Mexico $\operatorname{Tech}^{156,157}$ found that resistance factors (effective viscosities) provided by aluminum-citrateHPAM colloidal dispersion gels within cores (i.e., beyond the inlet core section) were the same as those provided by polymer solution alone (i.e., containing no crosslinker). This observation suggests that either the gel particles were too small to interact significantly with pore throats or the crosslinking reaction did not take place to a significant extent. These possibilities are quite consistent with the behavior for other gels. If insufficient polymer or crosslinker is present, gel formation will not take place (even small gel particles may not form). With such low aluminum concentrations (as low as $15 \mathrm{mg} / \mathrm{L}$ ), it is not surprising that a small (and expected) loss of crosslinker (e.g., by ion exchange or precipitation) could preclude formation of adequate gel particles.

Other Lab Tests Show Permeability Reductions, But Gelation Times Are Short. In other cases, ${ }^{155,156} \mathrm{CDG}$ gelants were found to penetrate into sand packs or sandstone and ultimately provide significant residual resistance factors. However, relatively short gelation times (less than one day) preclude deep penetration into reservoirs (i.e., over the course of weeks or months). ${ }^{155,156}$

Parallel Corefloods Are a Poor Indicator of Diversion. Representing a vendor of the colloidal dispersion gels, Smith et al. ${ }^{117}$ argued in contradiction to the results published by the above independent laboratories. The work of Smith et al. focused on parallel linear corefloods, ${ }^{117}$ which can be easily manipulated and misinterpreted as suggesting successful fluid diversion. ${ }^{15}$ These laboratory test results cannot be directly translated to profile modification in radial flow (i.e., unfractured wells). ${ }^{13,17,129}$ Also, with short laboratory cores (as used in the above laboratory study), diffusion and dispersion can readily compromise small gelant banks placed in the less permeable of the parallel cores - giving the false impression that gelant does not significantly enter or damage less-permeable oil zones. ${ }^{129}$ In real field applications, the distance of gelant penetration is several feet or more, even in the least-permeable oil-productive zones. ${ }^{13,17,129}$ For these distances, diffusion and dispersion will not destroy gelant banks. ${ }^{129}$

The Vendor's Lab Tests Show No Surprises. Smith et al. ${ }^{117}$ also argued that the aluminumcitrate-HPAM gelants can effectively propagate deep into porous rock and still provide greater residual resistance factors (permeability reduction factors) than polymer alone. However, in their lab study, all gelant was injected (mostly at high rates) in less than 2.5 hours after gelant preparation. No internal pressure taps, along the core material length, were used in their work, so the degree of injection face-plugging versus in-depth gel propagation could not be assessed. Smith et al. argued that in-depth propagation was demonstrated because some of the effluent from the cores eventually formed gels. However, rapidly injected gelant would be expected to propagate through short cores and still form gels. Their results did not indicate that gelant (polymer AND crosslinker) would propagate for days, weeks, or months through porous rockas required, if CDG flooding were to replace polymer flooding. Furthermore, their results did not 
indicate that gel will form and reduce permeability deep in the reservoir (beyond that caused by the polymer alone). As mentioned above, data from independent laboratories indicate that aluminum-citrate-HPAM gels of the type discussed in Refs. 116-118,154 will not propagate deep into porous rock and still provide greater residual resistance factors than polymer alone.

Requirements for a Viable Colloidal Dispersion Gel. If one wished to develop and demonstrate positive behavior for a suspension of gel particles, a number of useful and informative experiments should be performed. First, experiments should be performed using cores with multiple sections (e.g., multiple internal pressure taps along the core's length). The gelant formulation must (1) be injectable into the rock without causing progressive plugging of the inlet sand face, (2) show uniform resistance factors and residual resistance factors along the entire length of the core, (3) propagate these resistance factors through the porous rock at an acceptable rate (i.e., no excessive chemical retention), AND (4) provide greater resistance factors/residual resistance factors than polymer solution alone. During this series of floodingexperiment studies, one must also be concerned about the magnitude of residual resistance factors as a function of rock permeability. ${ }^{15,18-21}$ This latter comparison should be made using cores that were completely filled with polymer or gel (i.e., NOT using misleading parallel corefloods). If residual resistance factors in low-permeability rock are significantly greater than in high-permeability rock, polymer or gel treatments will impair sweep efficiency (see Fig. 46 and Table 2). ${ }^{13,17}$

\section{Examination of Field Data}

The Field and Lab Data Are Right. Only Interpretations Are Wrong. To CDG customers, the most influential claim made by those who advocate use of colloidal dispersion gels is that "successful" field applications demonstrate their utility. ${ }^{116-118,154}$ The CDG vendor suggested that a discrepancy exists between laboratory and field results - and that field results justify their untenable explanations. However, Darcy's Law, principles of polymer flooding, and the behavior of gelants and gels in porous media are very well established. Proposed explanations for field results should not contradict these principles without good reason.

If Darcy's Law and independent and well-documented laboratory results are accepted, two lines of reasoning remain to be investigated for explaining CDG field results. One line of reasoning is that CDG gels provided a benefit through some unrecognized mechanism. The challenge here is to identify any possible new mechanism. The second line of reasoning is that the aluminum citrate provided no significant benefit, even though some benefit may have come from using the polymer. In other words, is it possible that the aluminum citrate gave no incremental benefit over using the polymer alone? We are investigating both possibilities.

Field Results Can Be Explained Using More Plausible Concepts. In our examination of CDG projects in the United States and elsewhere, we have yet to see a convincing case that supports the untenable claims made by Chang and the CDG vendor (i.e., that CDG gels act as superpolymer solutions and that CDG gels preferentially enter high-permeability strata and later divert water into low-permeability zones). Instead, credible explanations can be envisioned that are consistent both with field and laboratory findings.

In general, the CDG field results fit into one of several categories. In the first field-results 
category, the treated reservoirs (with matrix rock permeabilities less than 10,000 md) contained no fractures or fracture-like flow features. For these cases, the aluminum was probably either removed (by adsorption on rock) or, for some other reason, did not crosslink the polymer. So the polymer alone could propagate (in an uncrosslinked form) through the formation and provide some benefit as a polymer flood. This scenario is very consistent with the laboratory findings from the University of $\mathrm{TX}^{124}$ and the University of Kansas. ${ }^{126}$ Since the aluminum provided no benefit for this scenario, the money spent on aluminum citrate could have been more effectively spent on more polymer.

In the second field-results category, the treated wells contained fractures or fracture-like flow features (e.g., vugs, karst, very-permeable conglomerate), even though the operator or gel vendor may not have been aware of these high-permeability flow features before the treatments. For these cases, the gels may have provided some benefit by partially plugging the fractures, fracture-like features, or high-permeability anomalies. However, for moderate to wide fractures, other types of gels [e.g., Cr(III)-acetate-HPAM)] probably would have been more effective. On a positive note for narrow (e.g., micro) fractures, colloidal dispersion gels conceivably may be more effective than other gels because of more effective penetration into tight fractures. ${ }^{130,131}$ This possibility requires further investigation.

In the third field-results category, the treated wells experienced general plugging of all open zones and flow paths. From an areal view, these wells were key to water channeling. By reducing the flow capacity of the treated wells, areal pressure gradients were altered so water injected into other (non-treated) wells pushed incremental oil toward offset production wells. This benefit could be realized by any means that reduced the flow capacity of the treated wells-including just choking back the injection rate of the well. Injection-well flow capacity can usually be reduced more cost-effectively and more easily using methods other than the CDG technology.

In the fourth field-results category, the reported benefits and/or increases in oil recovery had nothing to do with the colloidal dispersion gels. In some cases, the reported benefits for particular wells and fields occurred because of other changes or improvements that were implemented. In other cases, no real benefit occurred. The reported benefit was an artificial result of an overly pessimistic projection of the pre-treatment decline curve and/or an overly optimistic assignment of incremental oil to the gel treatment.

Daqing Field Results Are Consistent with No Benefit from Aluminum Citrate. Careful, objective analysis of the Daqing CDG field data suggest no credible, unambiguous improvement of the "CDG" flood over the normal polymer flood (especially see Tables 13 and 15 and Figs 3, 4, 10, and 11 in Ref. 116). Injectivity behavior was not significantly different for the two cases. Also, water/oil ratios and production trends cannot be credibly or unambiguously distinguished for the two processes. These results are consistent with the first category of field results mentioned above: the aluminum was either removed (by adsorption or precipitation) or was present in concentrations too low to allow polymer crosslinking within the reservoir.

In Table 13 of Ref. 116, Chang listed resistance factors of $\mathbf{1 . 3 1 6}$ (increasing from 1.120 to 1.512) during injection of a $0.179 \mathrm{PV}$ CDG Slug 1, 1.50 during injection of a subsequent $0.155 \mathrm{PV}$ polymer bank (with the same polymer concentration), and $\mathbf{1 . 5 3 7}$ during injection of a final 0.196 
PV CDG Slug 2. Chang ${ }^{116}$ claimed "indeed, the CDG solution formed strong resistance deep in the reservoir." Several serious problems are evident with this statement. First, it is not credible that resistance factors could be measured to three decimal places using field data - or even using lab data. Measurements accurate to 1 decimal place are normally the best that could be hoped for. Second, it is not credible that a resistance factor of 1.50 is significantly different from 1.512 or 1.537. (In truth, considering normal field data, it is difficult to believe that a value of 1.5 is statistically different from 1.3). Third, why was the resistance factor only around 1.5 when the solutions injected had viscosities around $20 \mathrm{cp}$ ? Why wouldn't the resistance factor be at least 20? The fact that the resistance factors were so low suggests a deficiency in the method of measurement.

The similarity of behavior during polymer and CDG (polymer with aluminum citrate) injection was evident in many of the tables and figures in Ref. 116 (see Tables 13 and 15 and Figs 3, 4, 10, and 11). These observations suggest that the aluminum citrate provided no benefit over normal polymer flooding.

More Convincing Field Data Needed. Undoubtedly, Chang and the CDG vendor that he represents feel that their CDG process works better than polymer floods and that we have missed some important mechanism of action during this discussion. We encourage them to pursue and demonstrate their point. However, future demonstrations should include two important aspects. First and foremost, explanations for how the process works should be consistent with wellestablished physical principles (e.g., Darcy's Law). Second, if they feel that field results are demonstrating some new physical principle, the field results should be less ambiguous than those presented in the past.

\section{Searching for a New Mechanism of Action for CDG Gels Does Shearing Of Colloidal Dispersion Gels Allow For Effective, Deep Penetration?}

Vendor Speculation. The CDG vendor speculated that when CDG gelants are sheared at high velocities in rock near a wellbore, gelation is delayed substantially (i.e., by weeks or months) thus, allowing gelant to penetrate far into a formation before developing high resistance when flowing at low velocities. ${ }^{117}$ This was pure speculation-no credible data was provided to support the suggestion.

First Test: Polymer Solution Injection. To test the vendor's hypothesis, we performed two sets of two-part experiments. CDG formulations were prepared that contained 300-ppm HPAM (Tiorco HiVis 350 ${ }^{\mathrm{TM}}$ ), 15-ppm aluminum (as citrate, Tiorco $677^{\mathrm{TM}}$ ), and $0.5 \% \mathrm{KCl}$. All experiments were performed at $41{ }^{\circ} \mathrm{C}$. In the first set of experiments, 300-ppm HPAM (without aluminum) were forced through a 493-md, 0.43-ft-long (5.2-inches) Berea core using a superficial velocity of $143 \mathrm{ft} / \mathrm{d}$. This core had one internal pressure tap that was located 0.75 inches from the inlet sand face.

During the course of injecting $118 P V\left(3,800 \mathrm{~cm}^{3}\right)$ of polymer solution, the resistance factors (apparent viscosities) were fairly stable and about the same $(\sim 42)$ in both core sections (left side of Fig. 47). The polymer effluent from this short core was collected and then injected into a 234md, 4-ft-long Berea core using a much lower rate of $2.7 \mathrm{ft} / \mathrm{d}$. This core had four equally spaced internal pressure taps, dividing the core into five sections of equal length. When the polymer 
solution effluent from the short core was injected into the long core, resistance factors were fairly similar in all five core sections - averaging about 7 (left side of Fig. 48). Incidentally, resistance factors for HPAM solutions are well known to increase with increased velocity in porous media $^{132}$ - consistent with our value of 42 in the short core at $143 \mathrm{ft} / \mathrm{d}$ versus 7 in the long core at $2.7 \mathrm{ft} / \mathrm{d}$. This effect has been attributed to the viscoelastic character of the HPAM polymer. ${ }^{132}$

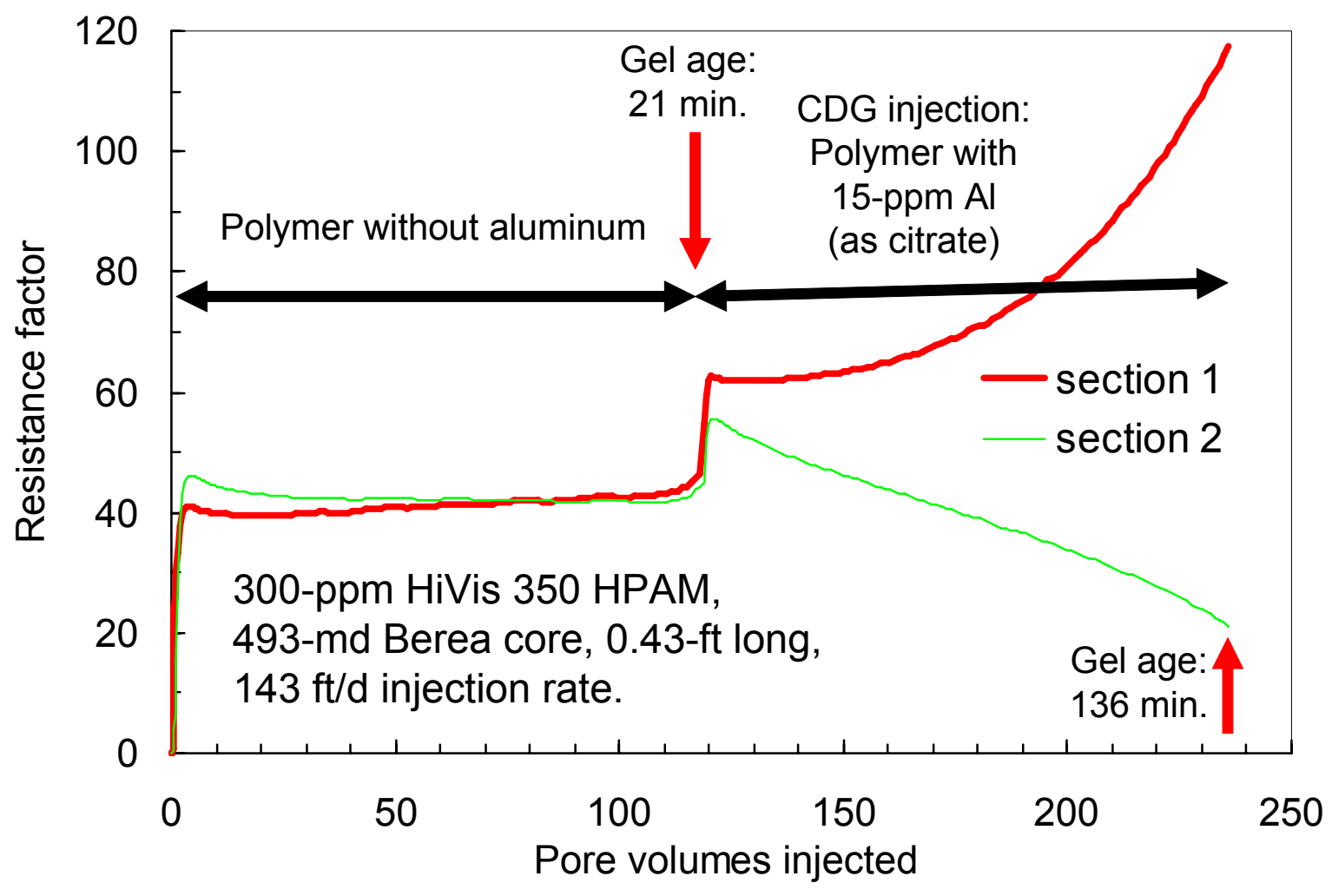

Fig. 47-Resistance factors in the first short core during injection at a high rate. 


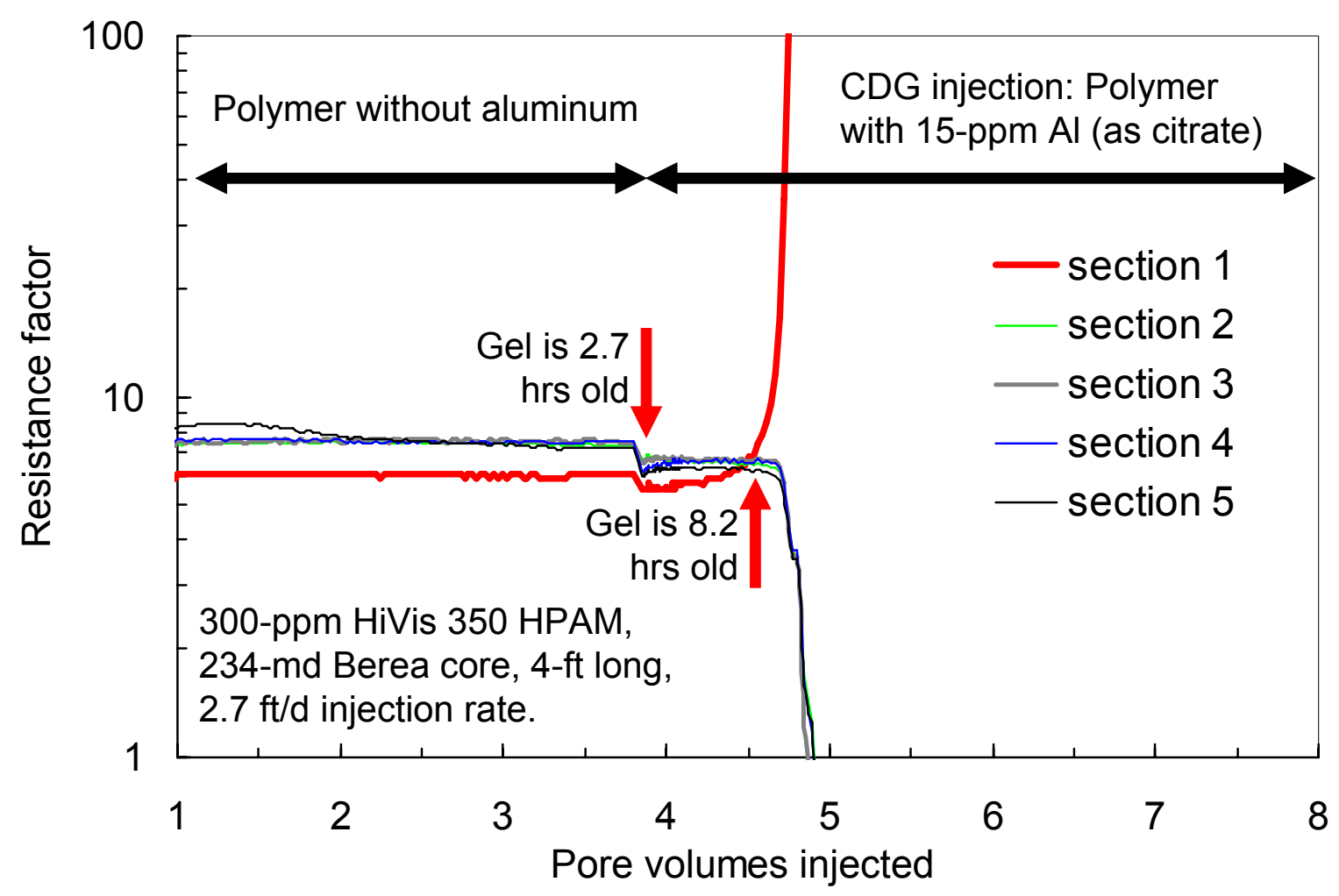

Fig. 48 - Resistance factors in the first long core during injection at a low rate.

First Test: CDG Injection. After injection of polymer solution, CDG formulation with the same polymer concentration (300-ppm HPAM) and with 15-ppm aluminum were injected into the 493-md, 0.43-ft-long Berea core, again at a rate of $143 \mathrm{ft} / \mathrm{d}$. For the first experiment (right side of Fig. 47), the CDG gel was 21 minutes old at the start of gelant injection and 136 minutes old at the end of gelant injection. During injection of $118 \mathrm{PV}$, the resistance factor increased to 117 in the first core section and decreased to 21 in the second core section. We concluded that some of the CDG gel was stripped from the solution during flow through the first core section.

CDG gelant that was effluent from the first core was 163 minutes $(2.7 \mathrm{hrs})$ old at the start of gelant injection into the 4-ft long core. For the next $5.5 \mathrm{hrs}(0.8 \mathrm{PV})$ of CDG injection (middle of Fig. 48), resistance factors remained stable in the five core sections. However, thereafter the resistance factor in the first core section quickly rose to a very high value, indicating severe faceplugging by the CDG gel. Simultaneously, resistance factors in the other four sections dropped to low values, indicating that the solution flowing through the downstream sections had been stripped of polymer. Thus, the concept advocated by the vendor does not appear to be valid.

Second Test: Polymer Solution Injection. To confirm the above results, these experiments were repeated. In the second set of experiments, 300-ppm HPAM (without aluminum) were forced through a 506-md, 0.43 -ft-long Berea core using a superficial velocity of $138 \mathrm{ft} / \mathrm{d}$. This core had one internal pressure tap that was located 0.83 inches from the inlet sand face. 
During the course of injecting $123 \mathrm{PV}\left(3,870 \mathrm{~cm}^{3}\right)$ of polymer solution, the resistance factors (apparent viscosities) were not as stable as those from the first set of experiments-steadily rising to 45 in the first section but decreasing to 28 in the second core section (left side of Fig. 49). The polymer effluent from this short core was collected and then injected into a 196-md, 4$\mathrm{ft}$-long Berea core using a much lower rate of $1.1 \mathrm{ft} / \mathrm{d}$. As in the first set of experiments, this core had four equally spaced internal pressure taps, dividing the core into five sections of equal length. When the polymer solution effluent from the short core was injected into the long core, resistance factors (left side of Fig. 50) were fairly similar in all five core sections - averaging about 6, close to the value of 7 observed during the first set of experiments (left side of Fig. 48).

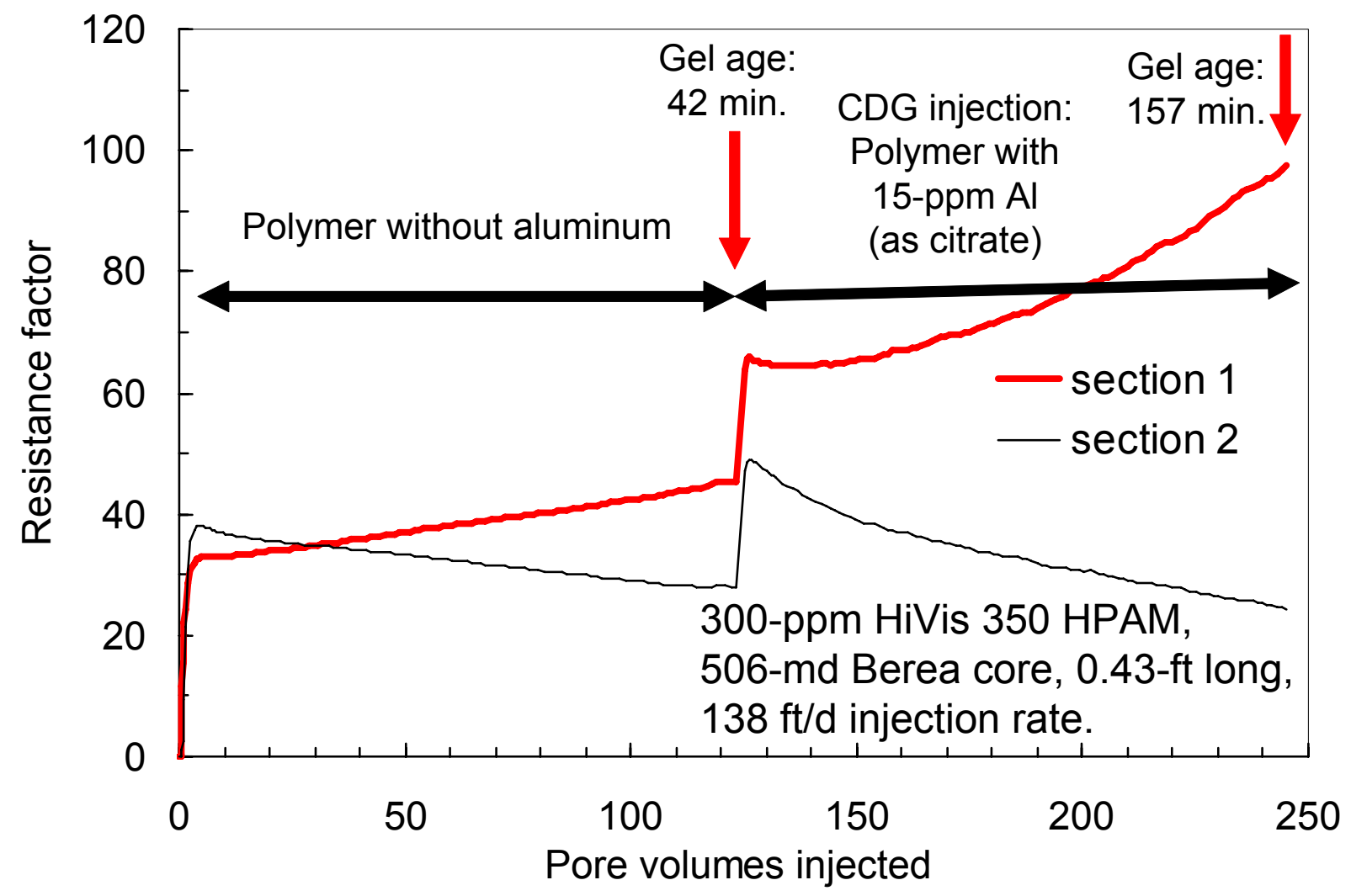

Fig. 49-Resistance factors in the second short core during injection at a high rate.

Second Test: CDG Injection. After injection of polymer solution, CDG formulation with the same polymer concentration (300-ppm HPAM) and with $15-\mathrm{ppm}$ aluminum were injected into the 506-md, 0.43 -ft-long Berea core, again at a rate of $138 \mathrm{ft} / \mathrm{d}$. For the first experiment (right side of Fig. 49), the CDG gel was 42 minutes old at the start of gelant injection and 157 minutes old at the end of gelant injection. During injection of $115 \mathrm{PV}$, the resistance factor increased to 98 in the first core section and decreased to 24 in the second core section. This behavior was similar to that seen during the analogous portion of the first set of experiments.

CDG gelant that was effluent from the first core was 192 minutes $(3.2 \mathrm{hrs})$ old at the start of gelant injection into the 4 -ft long core. For the next $5.7 \mathrm{hrs}(0.4 \mathrm{PV})$ of CDG injection (right side of Fig. 50), resistance factors remained stable in the final four core sections. However, the 
resistance factor in the first core section quickly rose to a very high value, indicating severe faceplugging by the CDG gel. After $0.4 P V$ of CDG injection, resistance factors in the other four sections dropped to low values, indicating that solution flowing through the downstream sections had been stripped of polymer. These results were reasonably consistent with those during the first set of experiments. Thus, the concept advocated by the vendor does not appear to be valid.

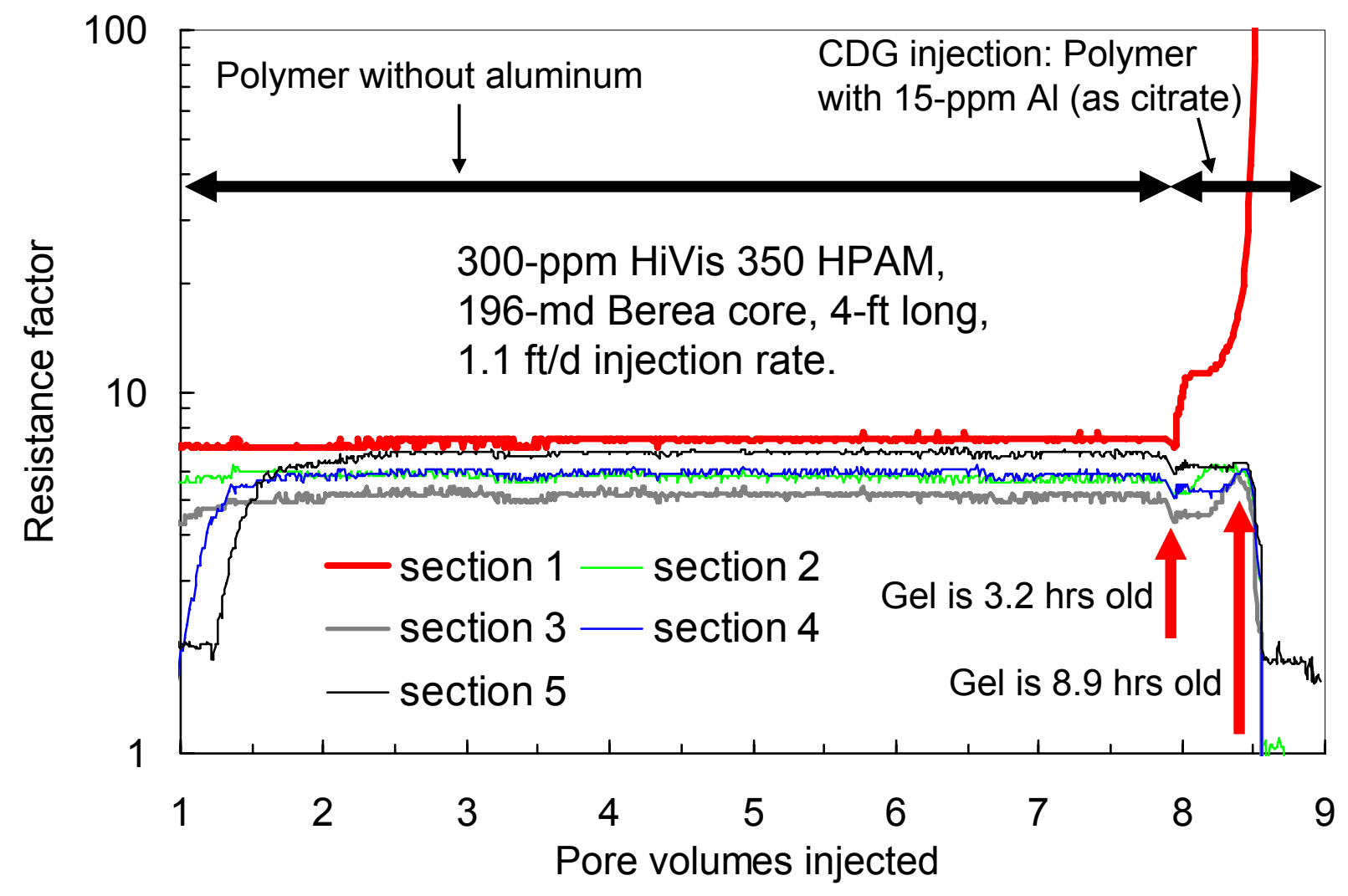

Fig. 50-Resistance factors in the second long core during injection at a low rate.

Conclusion. Shearing CDG gelants through porous rock may delay gelation and development of high resistance factors by a few hours, but certainly NOT for days, weeks, or months, as speculated (without support) by the CDG vendor. A relatively short time after gelant preparation ( 8.2 to $8.9 \mathrm{hrs}$ ), the sheared CDG gel caused severe plugging and did not propagate through 196to 234 -md rock.

\section{Can CDG Gels Flow in Porous Media with Permeabilities Less than 8 Darcys?}

Presumably, formed colloidal dispersion gels (or any other gel) could flow through a porous medium if the permeability was sufficiently high or if the pressure gradient was sufficiently large. For an extreme example, if the porous medium consisted of packed bowling balls, we suspect that many gels could readily be extruded through. Reservoir strata have been reported that have matrix permeabilities between 1 and 10 darcys-where fractures, vugs, and fracturelike features are not present. 
Experimental. We wondered whether formed CDG gels could enter and flow through a consolidated porous medium with a permeability up to 10 darcys. A 7.9-darcy porous polyethylene core was cast that was $2.6-\mathrm{ft}(78.4-\mathrm{cm})$ long and 1.55 inches $(3.94 \mathrm{~cm})$ in diameter. Porosity was $36.5 \%$. Four internal pressure taps were equally spaced along the core, creating five core sections of equal length. The core was saturated with brine $(0.5 \% \mathrm{KCl})$. All experiments were performed at $41^{\circ} \mathrm{C}$.

A colloidal dispersion gel was prepared that contained 300-ppm Tiorco HiVis350 HPAM, 15ppm aluminum (as citrate, Tiorco 677 ), and $0.5 \% \mathrm{KCl}$. This formulation was aged for one day at $41^{\circ} \mathrm{C}$. Then it was injected into the core using a rate of $26 \mathrm{ft} / \mathrm{d}\left(400 \mathrm{~cm}^{3} / \mathrm{hr}\right)$.

Results. Fig. 51 shows that resistance factors in the first core section rapidly rose and exceeded 1,000 during the first $0.08 P V$ of CDG injection. At this point (4 minutes after the start of injection), the throughput value for the inlet face was $2.2 \mathrm{~cm}^{3} / \mathrm{cm}^{2}$, and the pressure drop across the first core section was 179 psi. Thus, the gel caused severe face-plugging. In contrast, resistance factors for the other four sections of the core remained low-indicating no propagation of CDG gel beyond the first core section.

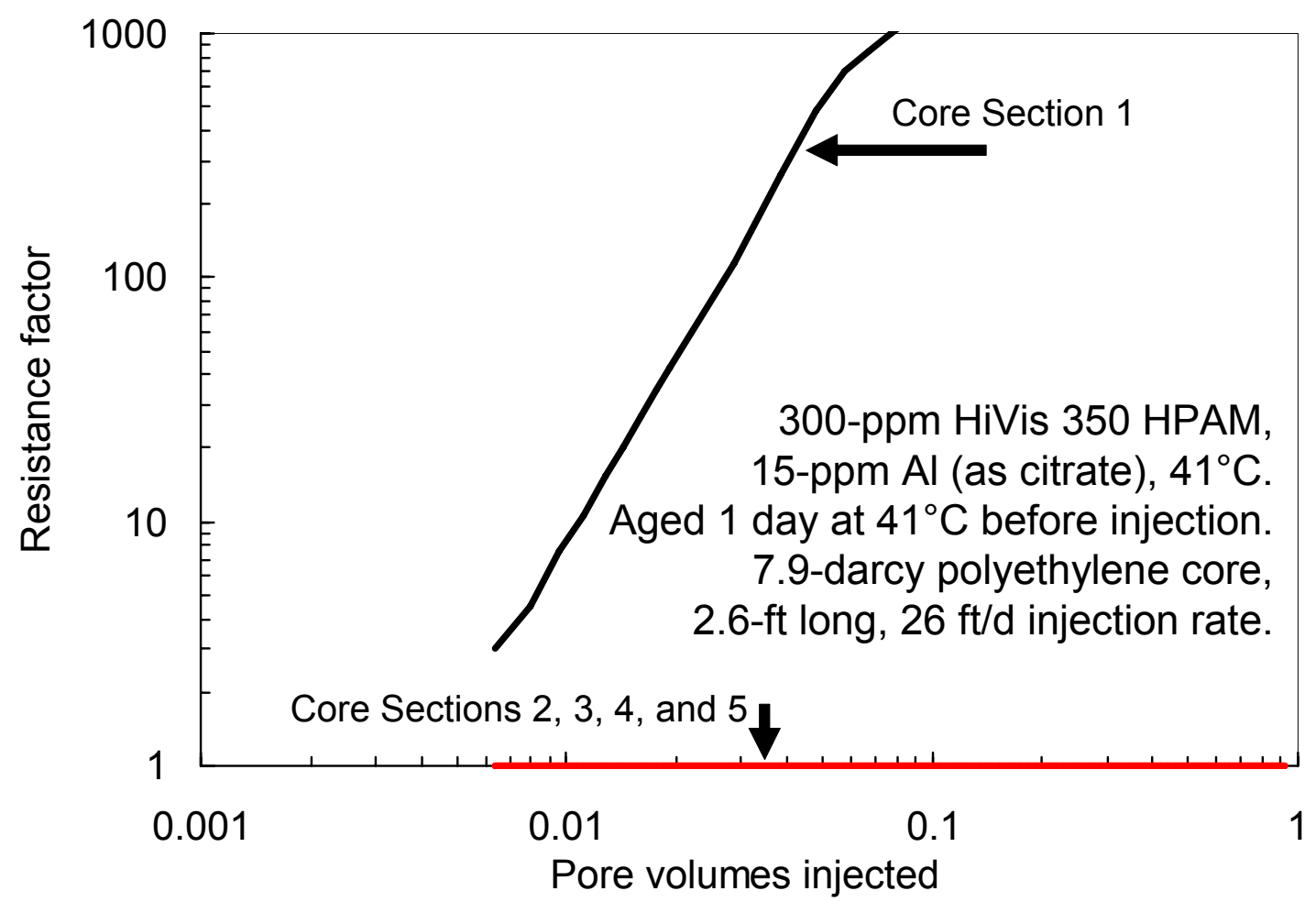

Fig. 51-Resistance factors for a 1-day-old CDG gel in a 7.9-darcy core.

Conclusion. One-day-old colloidal dispersion gels do not flow through porous media with permeabilities less than 8 darcys. 


\section{Can Low-Concentration Gels Be More Effective than Normal Gels in Tight Fractures?}

Can low-concentration gels penetrate into and plug narrow fractures more effectively than more concentrated gels? Our previous work revealed that the pressure gradient required to extrude a gel through a fracture varied inversely with the square of fracture width. ${ }^{72}$ For a one-day-old $\mathrm{Cr}$ (III)-acetate-HPAM gel with $0.5 \%$ Alcoflood $935,0.0417 \% \mathrm{Cr}$ (III) acetate, $1 \% \mathrm{NaCl}$, and $0.1 \% \mathrm{CaCl}_{2}$, the pressure gradient needed for extrusion through a 0.1 -mm-wide fracture was over $1,000 \mathrm{psi} / \mathrm{ft}$. During another set of experiments, we noted that a "partially formed" (i.e., gelant aged slightly longer than the gelation time) $\mathrm{Cr}(\mathrm{III})$-acetate-HPAM gel with $0.5 \%$ HPAM did not penetrate into a $0.05-\mathrm{mm}$-wide fracture with a pressure gradient of $65 \mathrm{psi} / \mathrm{ft} .{ }^{158}$ So for pressure gradients that are representative of field applications, $\mathrm{Cr}(\mathrm{III})$-acetate-HPAM gels with concentrations of $0.5 \%$ HPAM or more will not penetrate significant distances into narrow fractures.

We wondered whether low concentrations of gel might show value in penetrating into and plugging tight fractures more effectively than conventional gels with higher concentrations. (Baojun Bai et al. performed preliminary work investigating this idea ${ }^{130,131}$ ) We performed experiments using gels with four compositions: (1) $0.03 \%$ Tiorco HiVis $350 \mathrm{HPAM}$ and $0.0023 \% \mathrm{Al}(\mathrm{III})$ citrate $(0.0015 \% \mathrm{Al}),(2) \quad 0.15 \%$ Alcoflood $935 \mathrm{HPAM}$ and $0.0125 \% \mathrm{Cr}(\mathrm{III})$ acetate $(0.0028 \% \mathrm{Cr}),(3) 0.2 \%$ Alcoflood $935 \mathrm{HPAM}$ and $0.0167 \% \mathrm{Cr}(\mathrm{III})$ acetate $(0.0037 \%$ $\mathrm{Cr}$ ), and (4) $0.25 \%$ Alcoflood $935 \mathrm{HPAM}$ and $0.0209 \% \mathrm{Cr}(\mathrm{III})$ acetate $(0.0047 \% \mathrm{Cr})$. The first formulation contained $0.5 \% \mathrm{KCl}$ while the other three formulations contained $1 \% \mathrm{NaCl}$ and $0.1 \% \mathrm{CaCl}_{2}$. All experiments were performed at $41^{\circ} \mathrm{C}$. Fig. 52 shows viscosity versus shear rate for HPAM solutions with no crosslinker. Within the experimental error, the viscosities were fairly Newtonian for shear rates from 1 to $100 \mathrm{~s}^{-1}$, exhibiting average viscosities of $2.2 \mathrm{cp}$ for $0.03 \%$ HiVis 350 HPAM, $2.5 \mathrm{cp}$ for $0.15 \%$ Alcoflood 935 HPAM, $3.5 \mathrm{cp}$ for $0.2 \%$ Alcoflood $935 \mathrm{HPAM}$, and $6.5 \mathrm{cp}$ for $0.25 \%$ Alcoflood 935 HPAM. Incidentally, our interest in low concentration $\mathrm{Cr}(\mathrm{III})$-acetate-HPAM gels arose because of work performed in Argentina, ${ }^{159}$ where Tiorco appears to be applying the $\mathrm{Cr}(\mathrm{III})$-acetate-HPAM gels with similar objectives as those for their aluminum-citrate-HPAM "colloidal dispersion gels" (CDG). 


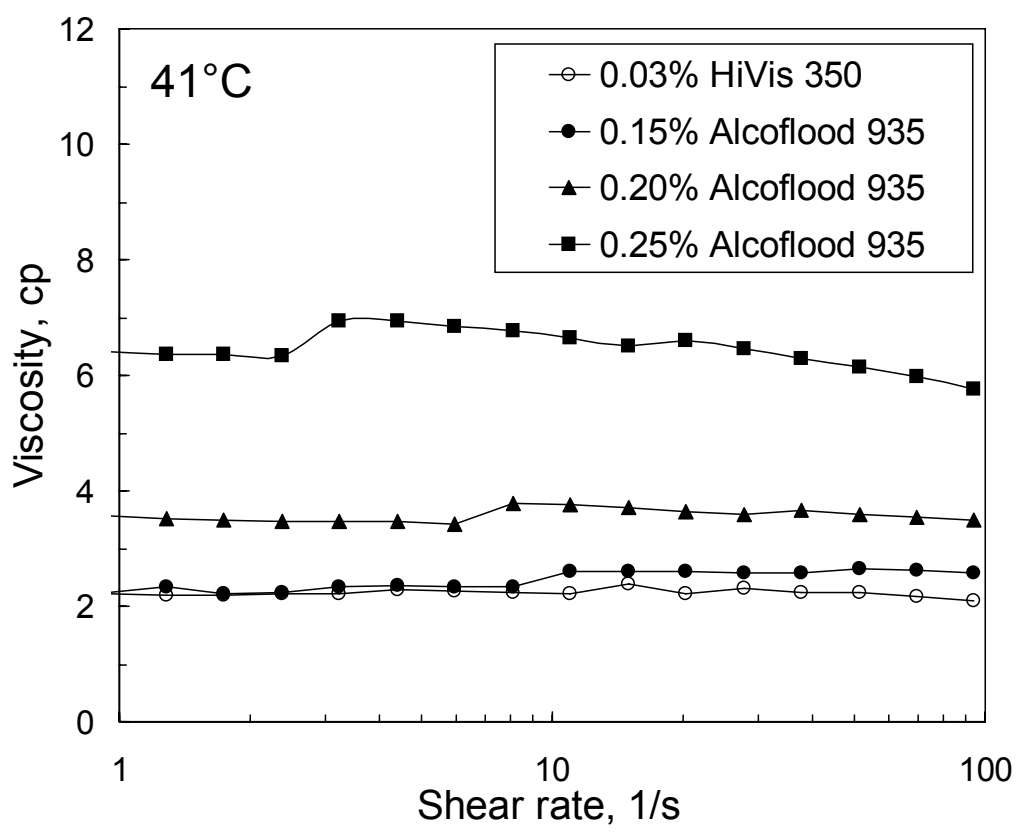

Fig. 52-Viscosity versus shear rate for HPAM solutions (no crosslinker).

With each gel composition, we performed experiments where $3,700 \mathrm{~cm}^{3}$ of one-day-old gel were extruded through fractures at $2,000 \mathrm{~cm}^{3} / \mathrm{hr}$. All fractures were $3.8 \mathrm{~cm}$ high in Berea sandstone cores that were $4-\mathrm{ft}(122-\mathrm{cm})$ long and $11.4 \mathrm{~cm}^{2}$ in cross-section. Four internal pressure taps (drilled into the fracture) divided the core into five sections of equal length $(0.8 \mathrm{ft})$. For each core, effluent could be produced from both the matrix and the fracture. The experiments used closed fractures, with calculated fracture widths ranging from 0.08 to $0.15 \mathrm{~mm}$. All fractures had smooth-sawed faces. After gel placement, brine was injected at a rate of $100 \mathrm{~cm}^{3} / \mathrm{hr}$. Table 14 summarizes the results.

Table 14-Use of low-concentration gels in tight fractures.

$L_{f}=4 \mathrm{ft}$, one-day-old gels, gel rate $=2,000 \mathrm{~cm}^{3} / \mathrm{hr}$, brine rate $=100 \mathrm{~cm}^{3} / \mathrm{hr}, 41^{\circ} \mathrm{C}$

\begin{tabular}{|l|c|c|c|c|}
\hline HPAM, \% & 0.03 & 0.15 & 0.2 & 0.25 \\
\hline HPAM type & HiVis 350 & Alcoflood 935 & Alcoflood 935 & Alcoflood 935 \\
\hline Crosslinker, \% & 0.0023 & 0.0125 & 0.0167 & 0.0209 \\
\hline Crosslinker type & $\mathrm{Al}(\mathrm{III})$ citrate & $\mathrm{Cr}(\mathrm{III})$ acetate & $\mathrm{Cr}(\mathrm{III})$ acetate & $\mathrm{Cr}(\mathrm{III})$ acetate \\
\hline Metal, \% & 0.0015 & 0.0028 & 0.0037 & 0.0047 \\
\hline$w_{f}, \mathrm{~mm}$ & 0.11 & 0.15 & 0.10 & 0.08 \\
\hline $\begin{array}{l}\text { Gel placement } \\
d p / d l, p s i / f t\end{array}$ & 56.7 & 4.9 & 27.8 & 71.8 \\
\hline $\begin{array}{l}\text { Effective } \mu \text { in } \\
\text { fracture, cp }\end{array}$ & 14.7 & 3.2 & 5.4 & 7.2 \\
\hline $\begin{array}{l}\text { Brine breaching } \\
d p / d l, \text { psi/ft }\end{array}$ & 3.2 & 0.19 & 29.7 & 72.1 \\
\hline $\begin{array}{l}\text { Final \% of flow } \\
\text { through matrix }\end{array}$ & 0 & 0 & 100 & 100 \\
\hline
\end{tabular}


Behavior during Gel Injection. As mentioned, the gel formulations were aged for one day before injection into the fractured cores at a rate of $2,000 \mathrm{~cm}^{3} / \mathrm{hr}$. Fig. 53 plots pressure gradient versus gel throughput (expressed in fracture volumes) for the four gels. Gel was detected at the fracture outlets after injecting from 3 to 10 fracture volumes. (One fracture volume $=4.6 \mathrm{~cm}^{3}$ if $w_{f}=0.1 \mathrm{~mm}$.) Between 10 and 500 fracture volumes of gel throughput, pressure gradients increased by 60 to $85 \%$ for the $\mathrm{Cr}$ (III)-acetate-HPAM gels. In contrast, for the Al(III)-citrateHPAM CDG gel, the pressure gradient increased by $460 \%$. The first data row below the solid line in Table 14 lists the average pressure gradients during gel extrusion. As expected, the average pressure gradient increased significantly with increased HPAM concentration for the $\mathrm{Cr}(\mathrm{III})$-acetate-HPAM gels. Interestingly, the average pressure gradient for the Al(III)-citrateHPAM CDG gel was quite high (56.7 psi/ft), considering that the gel contained only $0.03 \%$ HPAM. This result may have occurred because the molecular weight of HiVis 350 is significantly greater than that for Alcoflood 935.

The second data row below the solid line in Table 14 lists the effective viscosity exhibited by the gel in the fractures. For the $\mathrm{Cr}(\mathrm{III})$-acetate-HPAM gels, the effective viscosities were relatively low - on the order of the viscosities of the uncrosslinked polymer solutions (Fig. 52). These low effective viscosities should aid deep placement in narrow fractures. For the Al(III)-citrate-HPAM CDG gel, the effective viscosity in the fracture $(14.7 \mathrm{cp})$ was significantly greater than the polymer viscosity $(2.2 \mathrm{cp})$.

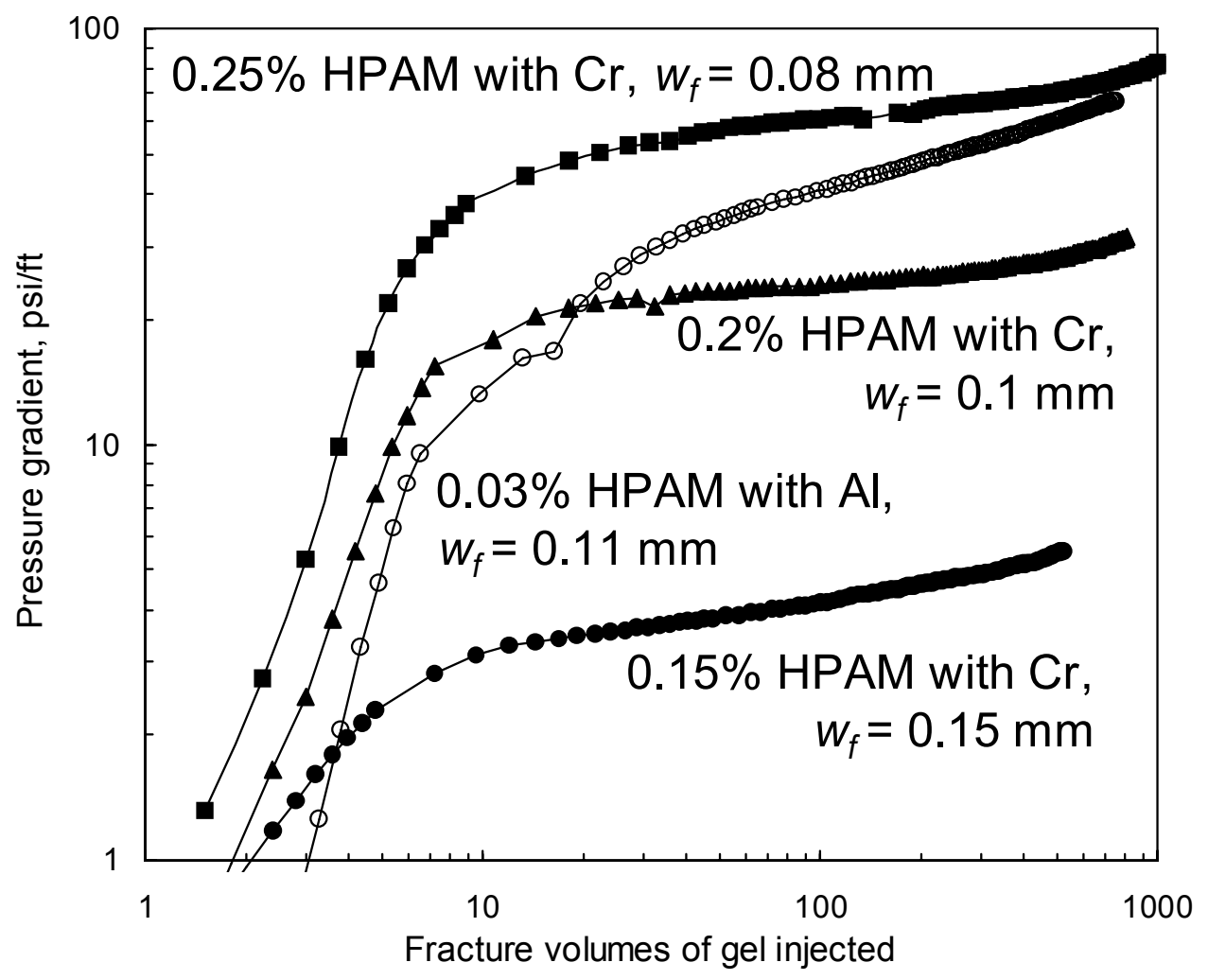

Fig. 53 - Pressure gradients versus volume throughput in tight fractures. 
Behavior during Brine Injection after Gel Placement. The second to last row in Table 14 lists the maximum pressure gradient observed during the first brine injection (at $100 \mathrm{~cm}^{3} / \mathrm{hr}$ ) after gel placement. This pressure gradient indicates the point where brine first breached the gel. ${ }^{160}$ For the two cases with $0.2 \%$ or more of HPAM, the brine breaching pressures were significant and on the order of the pressure gradients during gel placement. For the two cases with less than $0.2 \%$ HPAM, the brine breaching pressures were significantly less than the pressure gradients during gel placement.

The last row indicates that fraction of the injected brine that flowed through the matrix. The last row is particularly indicative of the gel diversion properties. Note that in two cases involving gels with $0.2 \%$ HPAM or $0.25 \%$ HPAM, $100 \%$ of the brine flow occurred through the matrix (i.e., $0 \%$ of the flow occurred through the fracture) after the gel treatment. For the other cases, little or no brine flowed through the matrix after the gel treatment. Thus, the two gels with higher HPAM concentrations were much more effective in plugging the tight fractures than those with the lower HPAM concentrations.

Conclusions. In fractures with widths around $0.1 \mathrm{~mm}$, one-day-old $\mathrm{Cr}(\mathrm{III})$-acetate-HPAM gels containing $0.15 \%, 0.2 \%$, or $0.25 \%$ HPAM propagated effectively, exhibiting effective viscosities that were similar to the viscosity of polymer solutions without crosslinker. In contrast, our previous work revealed that $\mathrm{Cr}$ (III)-acetate-HPAM gels with $0.5 \%$ HPAM would not enter these narrow fractures unless extremely high pressure gradients were applied. The gels containing $0.2 \%$ or $0.25 \%$ HPAM effectively healed these narrow fractures, forcing all post-gel-treatment brine to flow through the Berea sandstone matrix rather than the narrow fractures. Gels with lower HPAM concentrations were ineffective in preventing channeling through the fractures. An Al(III)-citrate-HPAM colloidal dispersion gel exhibited relatively high effective viscosity when extruding through a tight fracture and was ineffective in preventing channeling through the fracture. The Al(III)-citrate-HPAM gel appeared less attractive than Cr(III)-acetate-HPAM gels for treating tight fractures.

\section{Summary}

In summary, the suggestion ${ }^{116,154}$ that the CDG process was superior to normal polymer flooding was misleading and generally incorrect. Colloidal dispersion gels, in their present state of technological development, should not be advocated as an improvement to, or substitute for, polymer flooding. Laboratory results published by three independent universities confirm that CDG gelants and gels behave like other gels used in conformance control. In particular, before gelation (or before significant crosslinking occurs), gelants show the same flow properties in porous media as polymer solutions without crosslinker. After crosslinking, the gels do not flow through porous rock. Gelation times for CDG gels are fairly short-less than one day even at low temperatures. Shearing CDG gelants through porous rock at high velocities may delay gelation and development of high resistance factors by a few hours, but certainly NOT for days, weeks, or months, as speculated by the CDG vendor. One-day-old colloidal dispersion gels do not flow through porous media with permeabilities less than 8 darcys. 
We have yet to see a convincing case that supports the untenable claims made by the CDG vendor and their representative (i.e., that CDG gels act as super-polymer solutions and that CDG gels preferentially enter high-permeability strata and later divert water into low-permeability zones). Instead, credible explanations can be envisioned that are consistent both with field and laboratory findings. Careful objective analysis of the Daqing CDG field data suggest no credible, unambiguous improvement of the "CDG" flood over the normal polymer flood. Injectivity behavior was not significantly different for the two cases. Also, water/oil ratios and production trends could not be credibly or unambiguously distinguished for the two processes. These observations were consistent with laboratory results indicating that the aluminum was either removed (by adsorption or precipitation) or was present in concentrations too low to allow polymer crosslinking within the reservoir. 


\section{CAN FOAMS BE SUPERIOR TO POLYMERS FOR IMPROVING VERTICAL SWEEP EFFICIENCY IN RESERVOIRS?}

This chapter considers whether foams can be superior to polymers for improving vertical sweep efficiency in reservoirs. Our focus is on sweep improvement during waterflooding and chemical flooding rather than gas flooding. One special property of foams is their ability to collapse in low-permeability rock if capillary forces are sufficiently strong to drain water from foam films (i.e., the limiting-capillary-pressure effect). ${ }^{161-163}$ Under certain circumstances, this property may allow an injected gas/water/surfactant formulation to penetrate into low-permeability zones to a much greater extent than that using an aqueous polymer solution. Thus, vertical sweep could be more efficient during a foam flood than during a polymer flood. However, sweep efficiency could be poor within a low-permeability zone because of fingering associated with high mobility gas flow.

We first review vertical sweep behavior associated with polymer flooding in a two-layer reservoir with-versus-without crossflow. Then, we consider the distribution of flow for foam formulations compared with polymer solutions. Both linear and radial flow geometries are considered.

For simplicity, the following discussion will consider a two-layer reservoir. (The concepts presented here can easily be extended to more complex layering.) The most permeable layer will be called "Zone 1", while the second, less-permeable layer will be called "Zone 2." For a fluid injected to a distance, $L_{p 1}$ (for linear flow) or $r_{p 1}$ (for radial flow), in Zone 1, we are interested in the distance of fluid penetration, $L_{p 2}$ (for linear flow) or $r_{p 2}$ (for radial flow), in Zone 2. As a measure of vertical sweep efficiency, we are interested in the quantities, $L_{p 2} / L_{p 1}$ for linear flow or $r_{p 2} / r_{p 1}$ for radial flow. If one of these quantities has a value of unity, the injected fluid penetrates the same distance into both zones. In contrast, if the value is zero, no fluid penetrates into Zone 2.

\section{Normal Behavior of Viscous Fluids in Layered Reservoirs with No Crossflow}

Linear Flow. Consider a two-layer reservoir where an impermeable barrier separates the two zones. If flow is linear (e.g., leakoff into two zones that are cut by a vertical fracture) and if the injected fluid has the same mobility as the fluid being displaced, then the distance of injectant penetration should be proportional to the permeability/porosity ratio $(k / \phi)$ for a given zone. $^{6,17,129,164}$ For example (see Fig. 54), if Zones 1 and 2 have the same porosity but Zone 1 is ten times more permeable than Zone 2, the injectant flows 10 times farther in Zone 1 than in Zone $2\left(L_{p 2} / L_{p 1}=0.1\right)$. If the injected fluid is quite viscous, the distance of penetration should be roughly proportional to the square root of the permeability/porosity ratio for a given zone. $^{6,17,129,164}$ For example (see Fig. 55), if Zones 1 and 2 have the same porosity but Zone 1 is ten times more permeable than Zone 2, the injectant flows about three times farther in Zone 1 then in Zone $2\left(L_{p 2} / L_{p 1}=0.316\right)$. (Derivations of the equations for these calculations can be found in Appendix B.) For a spreadsheet that performs these calculations, download the file from: http://baervan.nmt.edu/randy/designing $\% 20$ gel $\% 20$ treatments/gelant $\% 20$ penetration $\% 20$ linear $\%$ 20flow1.xls 


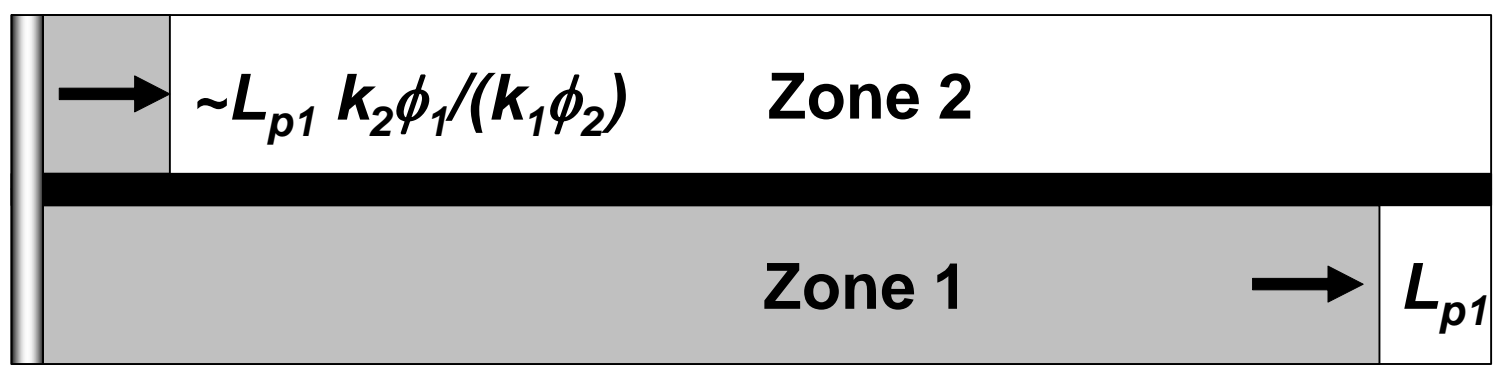

Fig. 54-Linear flow, no crossflow, unit-mobility displacement.

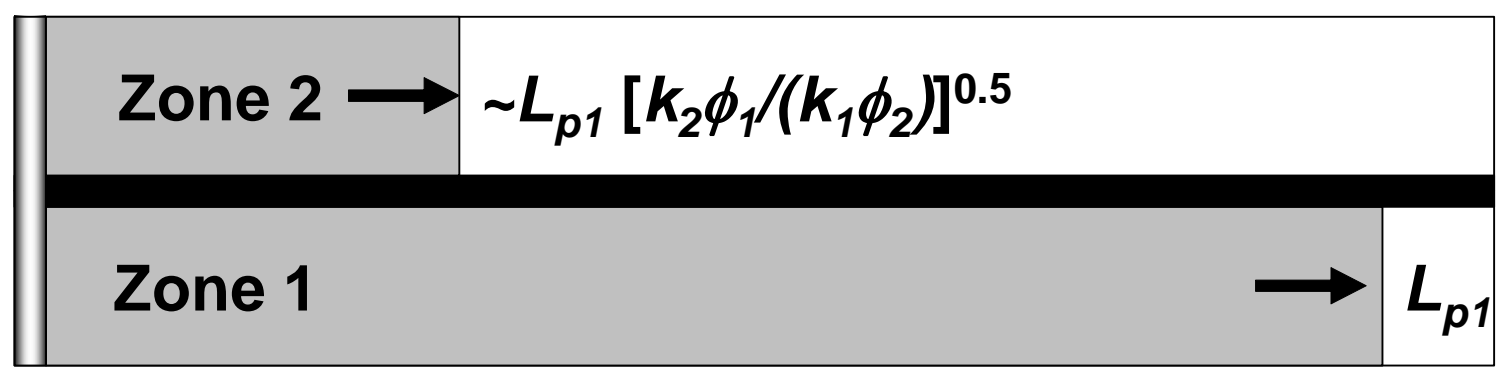

Fig. 55-Linear flow, no crossflow, normal Newtonian viscous fluid.

Radial Flow. For the case of radial flow with barriers to crossflow, ${ }^{6,17,129,164}$ the distance of penetration is roughly proportional to the square root of the permeability/porosity ratio for a given zone (Fig. 56). Certainly, more viscous fluids penetrate to a greater extent than lowviscosity fluids into low-permeability zones. Nonetheless, the square root of the permeability/porosity ratio provides a reasonable approximation for this case, regardless of injectant viscosity for normal Newtonian and non-Newtonian polymeric fluids. ${ }^{6,17,129,164}$ For a spreadsheet that performs these calculations, please download the file from our web site at: http://baervan.nmt.edu/randy/designing $\% 20$ gel $\% 20$ treatments $/$ gelant $\% 20$ penetration $\% 20 \mathrm{radial} \%$ 20flow1.xls.

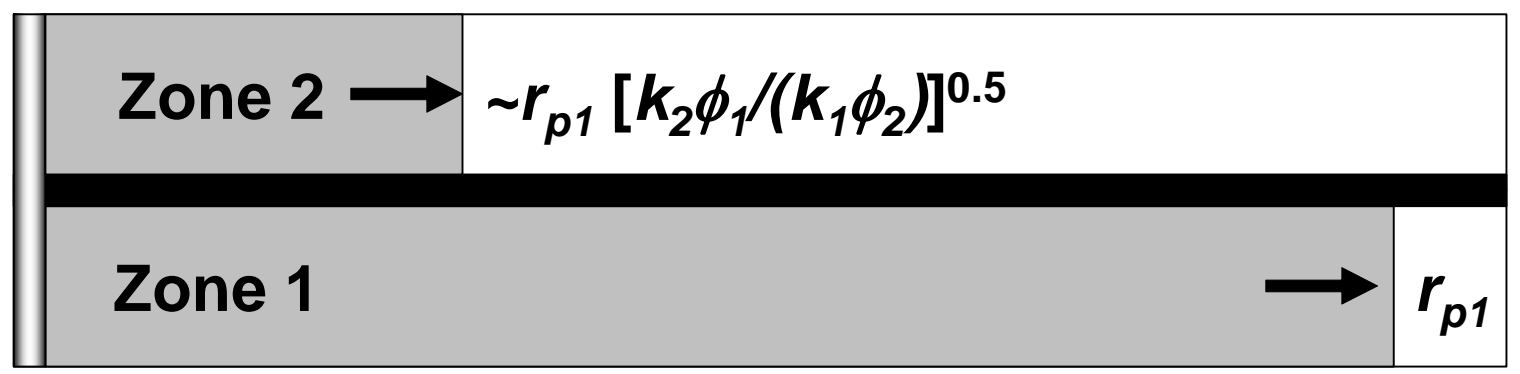

Fig. 56-Radial flow, no crossflow, Newtonian and non-Newtonian polymeric fluids.

\section{Normal Behavior of Viscous Fluids in Layered Reservoirs with Crossflow}

Unit-Mobility Displacement. Next, consider cases where fluids can freely crossflow between layers. For the case of linear flow and a unit-mobility displacement (e.g., a water-like fluid), the distance of injectant penetration should be proportional to the permeability/porosity ratio for a given zone- just as in the no-crossflow case (Fig. 57, compare with Fig. 54). For the case of 
radial flow and a unit-mobility displacement, ${ }^{112}$ the radius of penetration is roughly proportional to the square root of the permeability/porosity ratio for a given zone (Fig. 58).

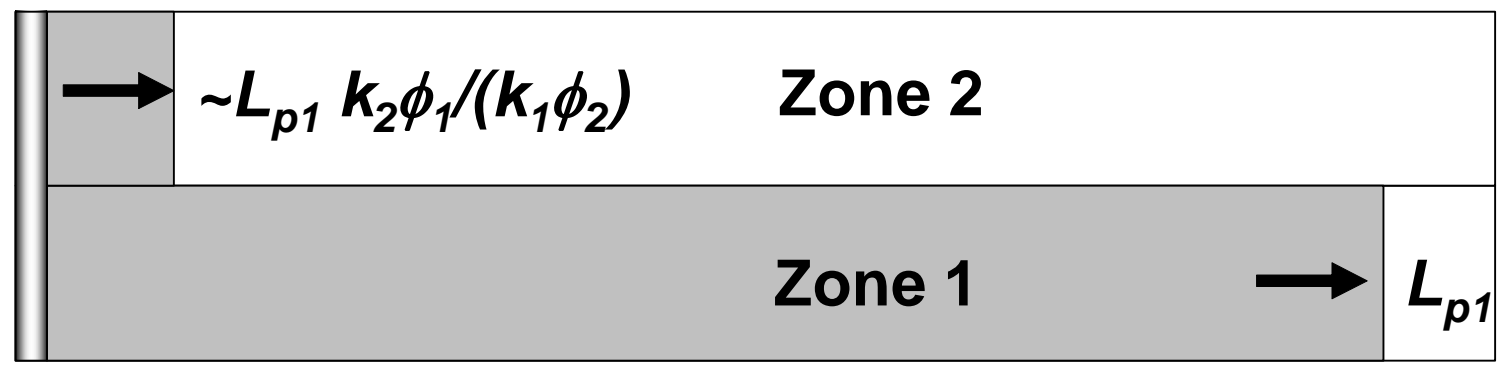

Fig. 57-Linear flow, free crossflow, unit-mobility displacement.

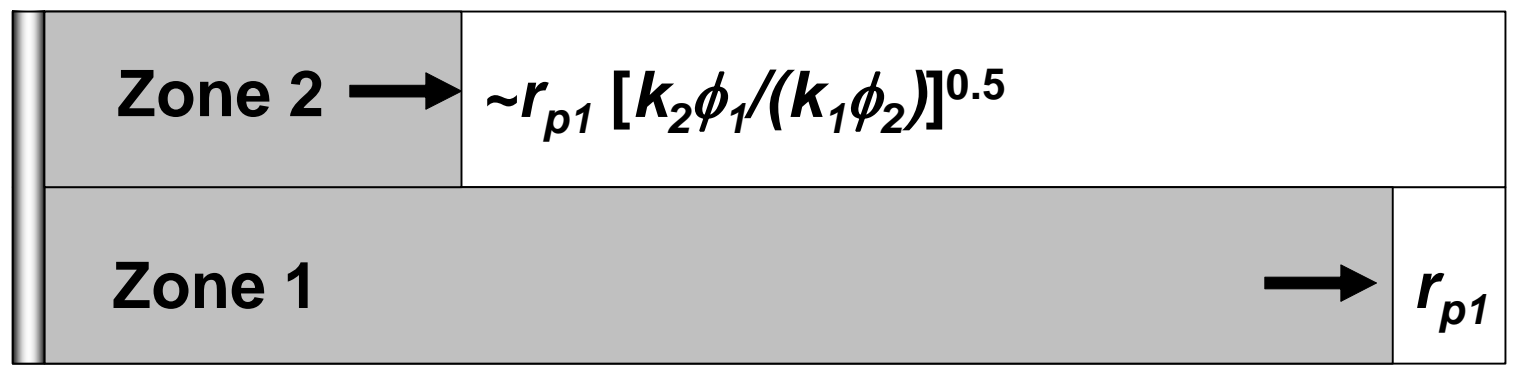

Fig. 58-Radial flow, free crossflow, unit-mobility displacement.

Normal Viscous Fluids, $\boldsymbol{F}_{\boldsymbol{r}}<\boldsymbol{k}_{1} \boldsymbol{\phi}_{2} /\left(\boldsymbol{k}_{2} \phi_{1}\right)$. Consider the case where the injectant is a normal viscous Newtonian or non-Newtonian polymeric fluid and fluids can freely crossflow. Also, let the resistance factor of the injectant $\left(F_{r}\right)$ be less than the permeability/porosity contrast for the two zones $\left(k_{1} \phi_{2} /\left(k_{2} \phi_{1}\right)\right)$. Then, for linear flow, the distance of injectant penetration should be proportional to the product of the resistance factor and the permeability/porosity ratio, ${ }^{112}$ as indicated in Fig. 59. For a spreadsheet that performs this calculation, download: http://baervan.nmt.edu/randy/designing $\% 20$ gel $\% 20$ treatments/gelant $\% 20$ penetration $\% 201$ inear $\%$ 20flow2.xls.

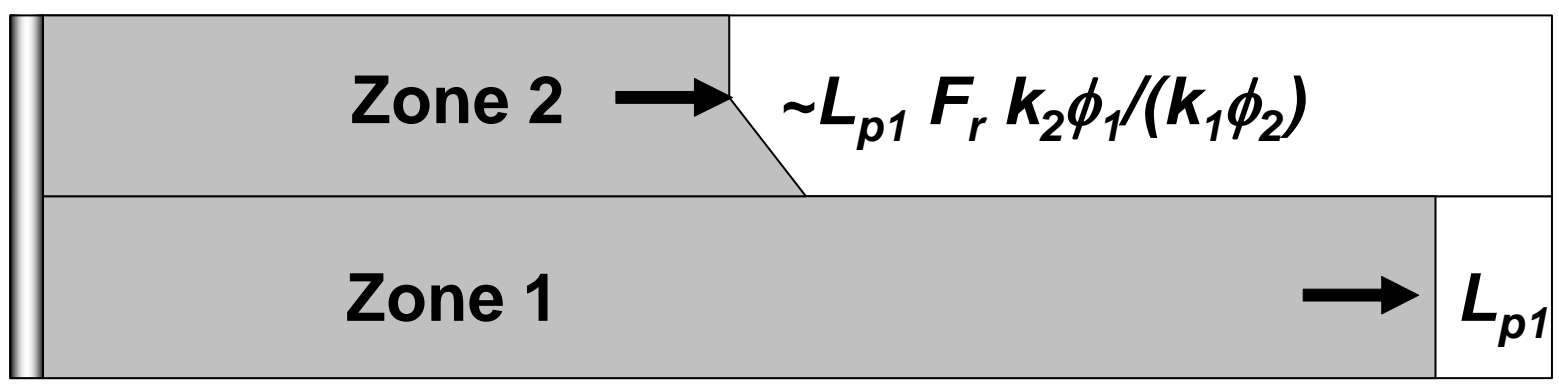

Fig. 59-Linear flow, free crossflow, $F_{r}<k_{1} \phi_{2} /\left(k_{2} \phi_{1}\right)$.

For radial flow, the distance of injectant penetration should be proportional to the square root of the product of the resistance factor and the permeability/porosity ratio, ${ }^{112}$ as indicated in Fig. 60. 
http://baervan.nmt.edu/randy/designing $\% 20$ gel $\% 20$ treatments/gelant $\% 20$ penetration $\% 20$ radial $\%$ 20flow2.xls provides a spreadsheet for this calculation.

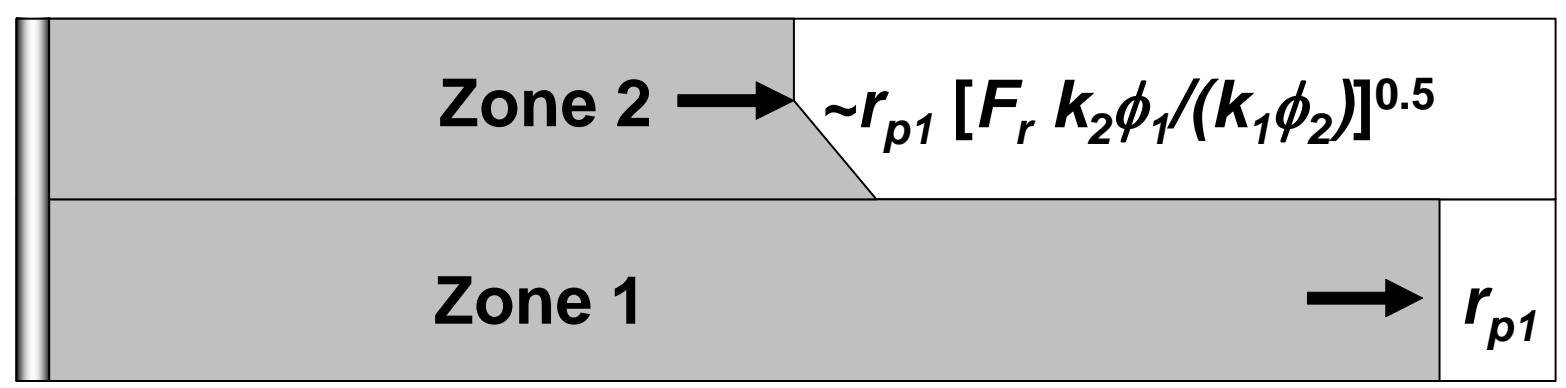

Fig. 60-Radial flow, free crossflow, $F_{r}<k_{1} \phi_{2} /\left(k_{2} \phi_{1}\right)$.

Normal Viscous Fluids, $\boldsymbol{F}_{r} \geq \boldsymbol{k}_{1} \phi_{2} /\left(\boldsymbol{k}_{2} \phi_{1}\right)$. Now consider the case where the resistance factor of a normal viscous fluid is greater than the permeability/porosity contrast, $F_{r} \geq k_{1} \phi_{2} /\left(k_{2} \phi_{1}\right)$. For this case, the distance of injectant penetration can be almost the same distance in both zones (Fig. 61). This observation is true for both linear and radial flow if free crossflow can occur for normal viscous fluids. ${ }^{12}$

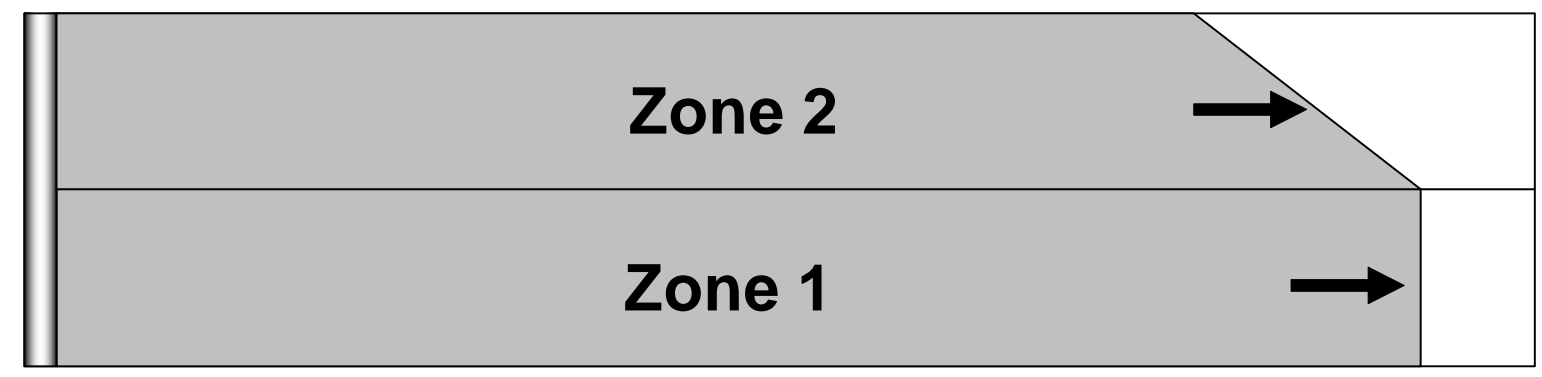

Fig. 61—Either linear or radial flow, free crossflow, $F_{r} \geq k_{1} \phi_{2} /\left(k_{2} \phi_{1}\right)$.

\section{Foam Flow}

Foam Forms in Both Zones. Now we consider cases where foam flows instead of polymer solutions or Newtonian fluids. If the foam forms in both zones, the cases illustrated in Figs. 5461 generally cover the range of possibilities. This statement assumes that the mobility of the foam (or of the injected polymer solution) is no greater than that of the oil/water banks that are being displaced.

Foam Forms in Zone 1, but Not in Zone 2. Free Crossflow. Under some circumstances, the limiting-capillary-pressure effect could allow a low-mobility foam to form in high-permeability zones, but not in low-permeability zones. In low-permeability zones, capillary forces may draw water from liquid films, minimizing bubble and foam formation. ${ }^{160-163}$ Consequently, the mobility of the gas/water/surfactant mixture could be quite high in low-permeability zones, while foam mobility remains low in high-permeability zones. 
If free crossflow can occur, the distance of penetration of the gas/water mixture in the lowpermeability zones (i.e., Zone 2 in our simple example) can be no greater than that in the highpermeability zone (Zone 1). If gas and water in Zone 2 attempt to outrun the foam bank in Zone 1, the fluids will simply crossflow back into Zone 1 near the foam front (see Fig. 62). Thus, sweep improvement from foams in this scenario is not expected to be significantly greater than that for viscous polymer solutions (Fig. 61).

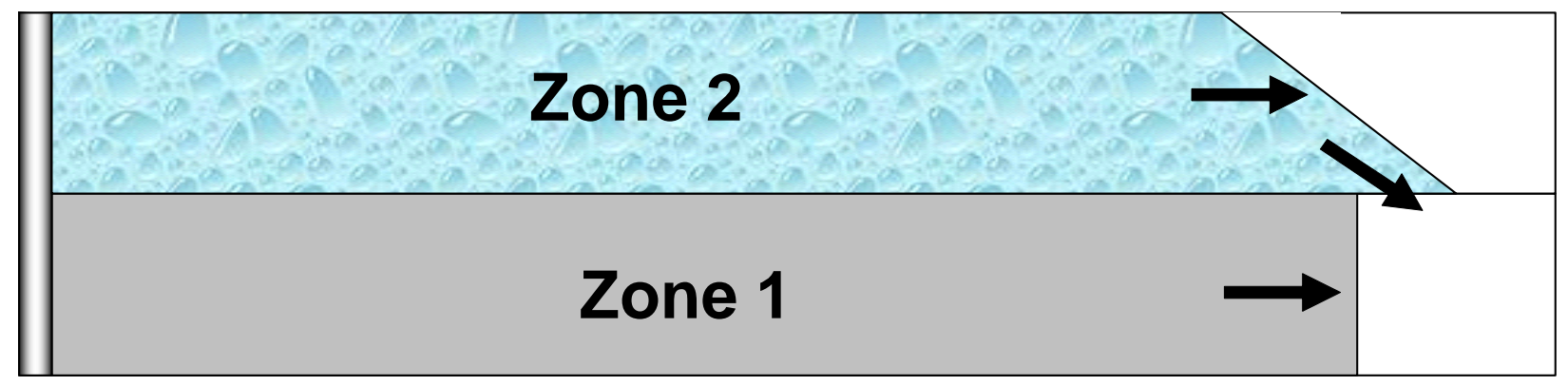

Fig. 62-Foam forms in Zone 1 but not in Zone 2, free crossflow.

Foam Forms in Zone 1, but Not in Zone 2. No Crossflow. We now come to the cases where foams are anticipated to provide superior mobility control to polymer solutions. These cases require that (1) foam forms in the high-permeability zones (i.e., Zone 1), (2) foam does not form in the low-permeability zones (i.e., Zone 2), and (3) no crossflow occurs between zones. If these conditions are met, upon first consideration, Fig. 63 illustrates an ideal scenario. That is, the foam bank in Zone 1 provides enough resistance to force the front of the gas/water/surfactant formulation in Zone 2 (the low-permeability zone) well beyond the foam front in Zone 1.

However, an important flaw exists with the picture illustrated by Fig. 63. In particular, the gas/water/surfactant formulation will have a high mobility and will form viscous fingers ${ }^{165}$ through the oil/water bank in Zone 2 (Figs. 64 and 65). The mobility of nitrogen (or a similar gas) might be 50 or more times greater than that for oil or water. In some cases (with dense phase $\mathrm{CO}_{2}$ or hydrocarbon gases), gas mobility might be only ten times greater than the mobility of oil or water. ${ }^{166}$ The severity of fingering increases with increasing mobility ratio. ${ }^{165}$ Inefficient displacement associated with these fingers will partially offset the improvement in vertical sweep provided by increased penetration of gas/water/surfactant formulation into Zone 2 .

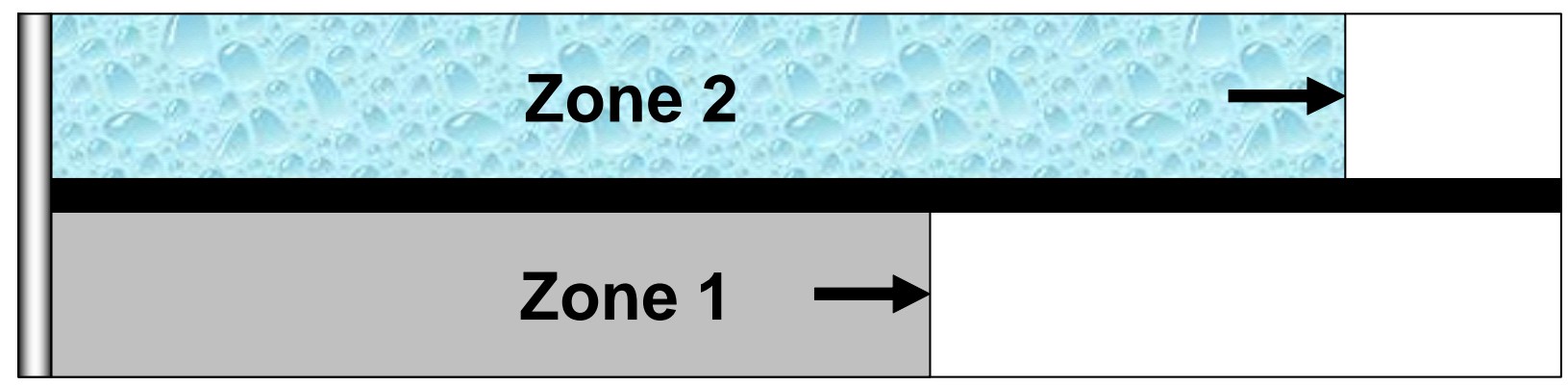

Fig. 63-Incorrect view of gas/water flow in Zone 2 after foam forms in Zone 1. 


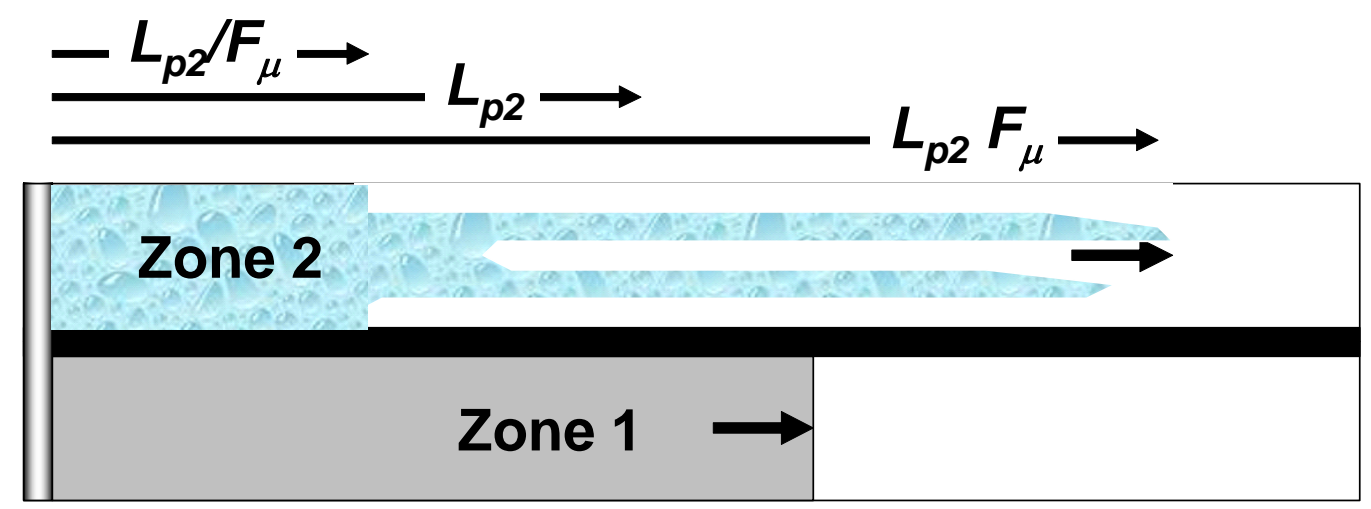

Fig. 64-Gas/water flow in Zone 2 after foam forms in Zone 1. No Crossflow. Linear flow.

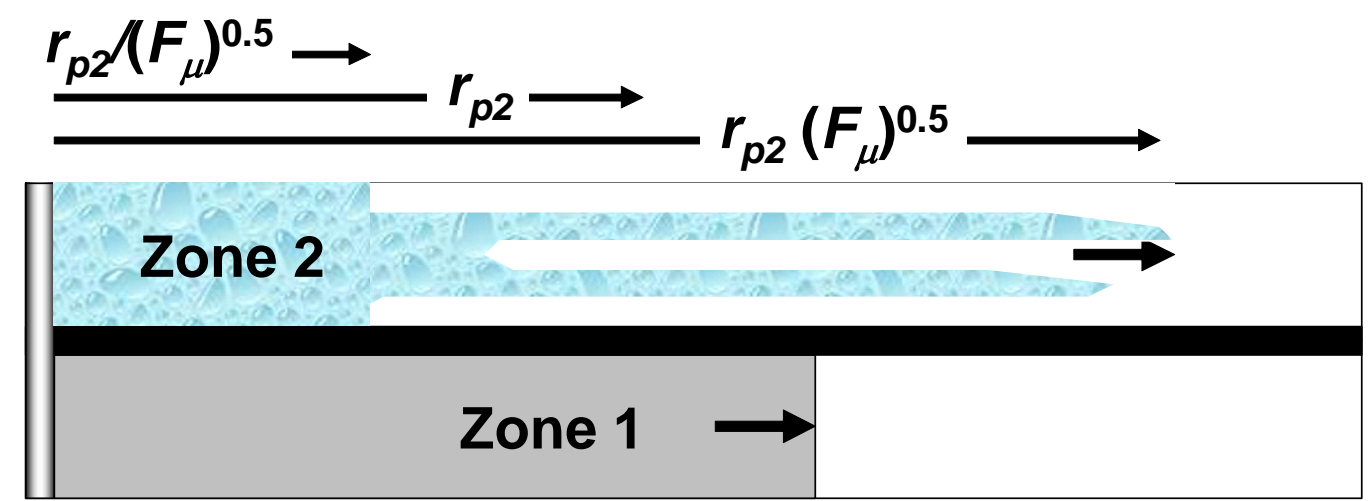

Fig. 65-Gas/water flow in Zone 2 after foam forms in Zone 1. No Crossflow. Radial flow.

Model for Unstable Displacement. Our objective now is to assess which effect will dominate sweep efficiency: (1) the positive effects associated with increased fluid diversion into Zone 2 by the foam or (2) the negative effects from the viscous fingers in Zone 2. To make this determination, we rely on the quarter-power mixing rule and the method of Koval. ${ }^{129,165}$ In this method, Zone 2 is divided into three regions. In the region farthest from the wellbore, the oil/water bank has a relatively constant saturation and fractional flow, with an average mobility of $\lambda_{o w}$. If Zone 2 is an unswept oil zone, oil will dominate the determination of this mobility. In the region closest to the wellbore, the gas/water/surfactant formulation has driven oil to a residual value and the average mobility is $\lambda_{g w}$. Since foams usually have high gas saturations and because gas is substantially more mobile than water, gas dominates the determination of this mobility. In the intermediate region, high-mobility gas forms viscous fingers through the oil/water bank. The average mobility in this region, $\lambda_{o w} F_{\mu}$, is estimated using the quarter-power mixing rule $^{165}$ (Eq. 43).

$F_{\mu}=\left[0.78+0.22\left(\lambda_{g w} / \lambda_{o w}\right)^{0.25}\right]^{4}$

If the total volume of gas/water/surfactant formulation that penetrates into Zone 2 can be determined, the bank sizes associated with each of the three regions can be estimated. ${ }^{17,129,165}$ From the total volume entering Zone 2 and the formation height and porosity, an average distance $\left(L_{p 2}\right)$ or radius $\left(r_{p 2}\right)$ of formulation penetration can be calculated. For linear flow (e.g., 
leakoff from fracture faces), the length of the gas/water region (see Fig. 64) in Zone 2 (i.e., the distance from the well or fracture face to the trailing edge of the fingering region) is given by Eq. 44 .

$L_{g w}=L_{p 2} / F_{\mu}$

For radial flow, the radius of the gas/water region (see Fig. 65) in Zone 2 is given by Eq. 45.

$r_{g w}=r_{p 2} /\left(F_{\mu}\right)^{0.5}$

For linear flow, the distance from the well or fracture face to the furthest extent of the fingering region (see Fig. 64) in Zone 2 is given by Eq. 46.

$L_{v f}=L_{p 2} F_{\mu}$

For radial flow, the radius from the well to the furthest extent of the fingering region (see Fig. 65) in Zone 2 is given by Eq. 47.

$r_{v f}=r_{p 2}\left(F_{\mu}\right)^{0.5}$

Given these equations, the methods from Refs. 17 and 129 can be used to estimate front positions and sweep efficiencies during foam injection under various circumstances. These methods simply employ the Darcy equations for flow in parallel and series. Appendix C provides derivations of the relevant equations.

\section{Results}

Two fronts are of interest in Zone 2. One is the trailing edge of the region of viscous fingering, and the other is the front (end) of the region of viscous fingering, as illustrated in Figs. 64 and 65. Figs. 66-71 show the positions of these fronts (relative to the foam front in Zone 1, $L_{p 1}$ or $r_{p 1}$ ) for various circumstances. The thick solid curves show the positions of the trailing edge of the viscous fingering regions, while the thin solid curves show the positions of the viscous fingering fronts. For comparison, the thick dashed curves show the behavior provided by polymer solution (10-cp, except for figures where resistance factor, $F_{r}$, is the variable).

In these figures, the finger fronts (the thin solid curves) are the most optimistic projections of sweep in Zone 2. The trailing edges of the fingering region (the solid curves) provide more realistic projections.

Linear Flow. Figs. 66-68 show behavior predicted for linear flow (e.g., leakoff from fracture faces). Fig. 66 considers cases where the permeability contrast $\left(k_{1} / k_{2}\right)$ is 10 and the foam resistance factor $\left(F_{r}\right)$ is 10 . Penetration into Zone 2 is plotted versus the ratio gas mobility $\left(\lambda_{g w}\right)$ to mobility of the oil/water bank $\left(\lambda_{o w}\right)$. If this ratio is greater than 10 (i.e., the value of the foam resistance factor in Zone 1), 10-cp polymer solutions provide a better vertical sweep (greater penetration into Zone 2) than the gas/water/surfactant formulation (compare dashed curve with the thick solid curve). 


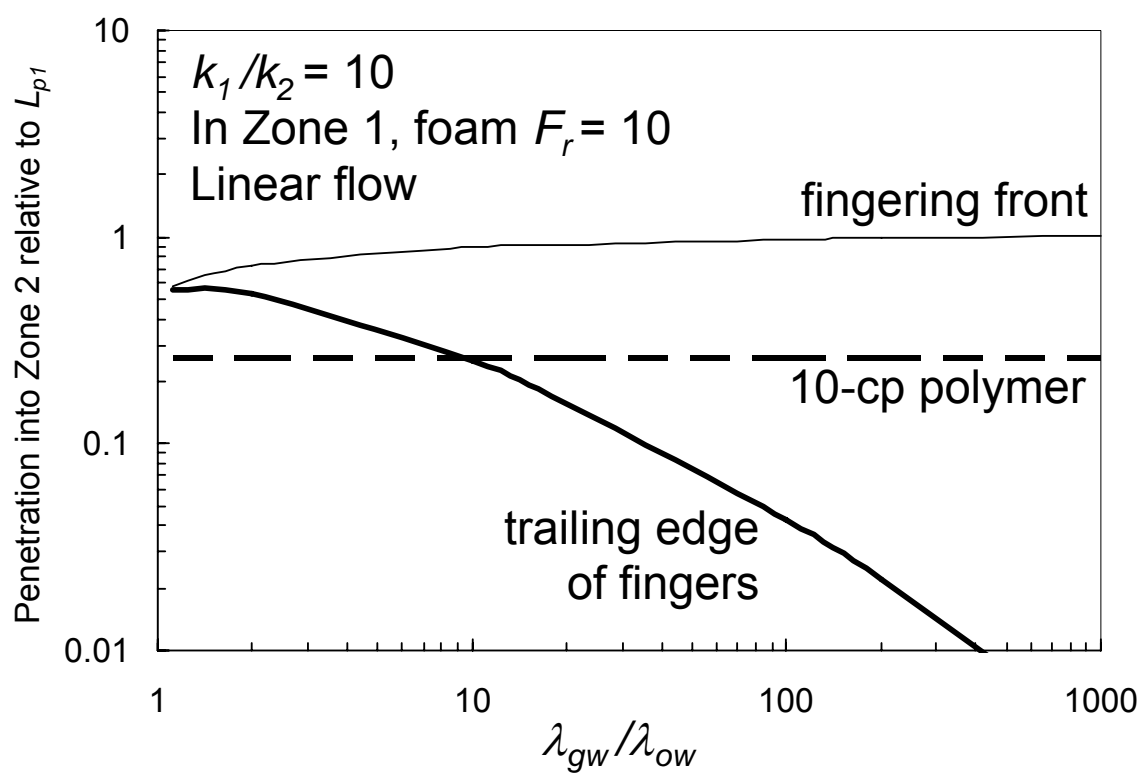

Fig. 66-Fronts in Zone 2 versus mobility ratio. Linear flow.

Fig. 67 shows the effects of resistance factor on vertical sweep for cases where the permeability contrast and the ratio, $\lambda_{g w} / \lambda_{o w}$, were both fixed at values of 10 . For the foam cases (thin and thick solid curves), the resistance factor in Zone 1 is indicated on the $x$-axis. In Zone 2, no foam forms, so front positions are determined by $\lambda_{g w}$ and $\lambda_{o w}$. For the polymer cases, the resistance factor indicated on the $x$-axis applies to both Zone 1 and Zone 2. Fig. 67 shows that foam resistance factor in Zone 1 has a major impact on vertical sweep. For the conditions given, polymer solutions provide better sweep for resistance factors below 10. For resistance factors above 10, vertical sweep favored the use of foams. In fact, for resistance factors above 100, gas/water/surfactant penetration into Zone 2 exceeded foam penetration into Zone 1.

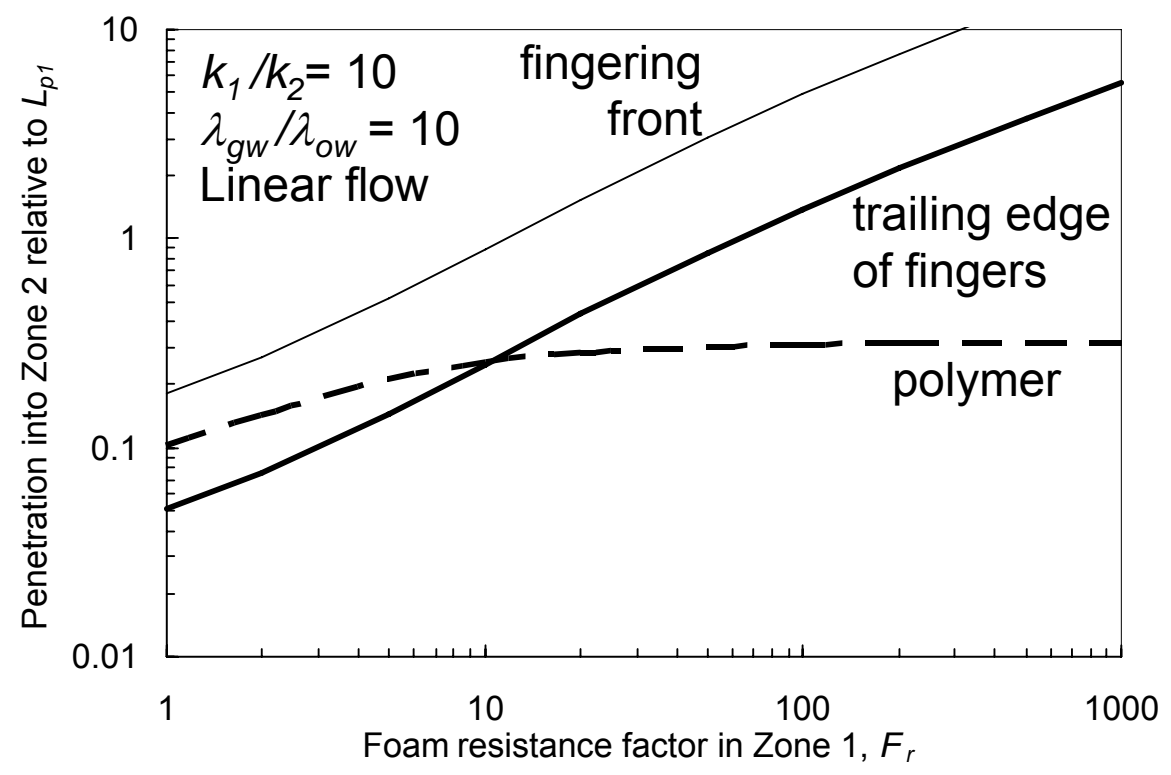

Fig. 67-Fronts in Zone 2 versus resistance factor in Zone 1. Linear flow. 
Fig. 68 shows the effects of permeability contrast on vertical sweep for cases where the resistance factor in Zone 1 and the ratio, $\lambda_{g w} / \lambda_{o w}$, were both fixed at values of 10 . For both foams and polymers, vertical sweep efficiency decreased significantly as permeability contrast $\left(k_{1} / k_{2}\right)$ increased.

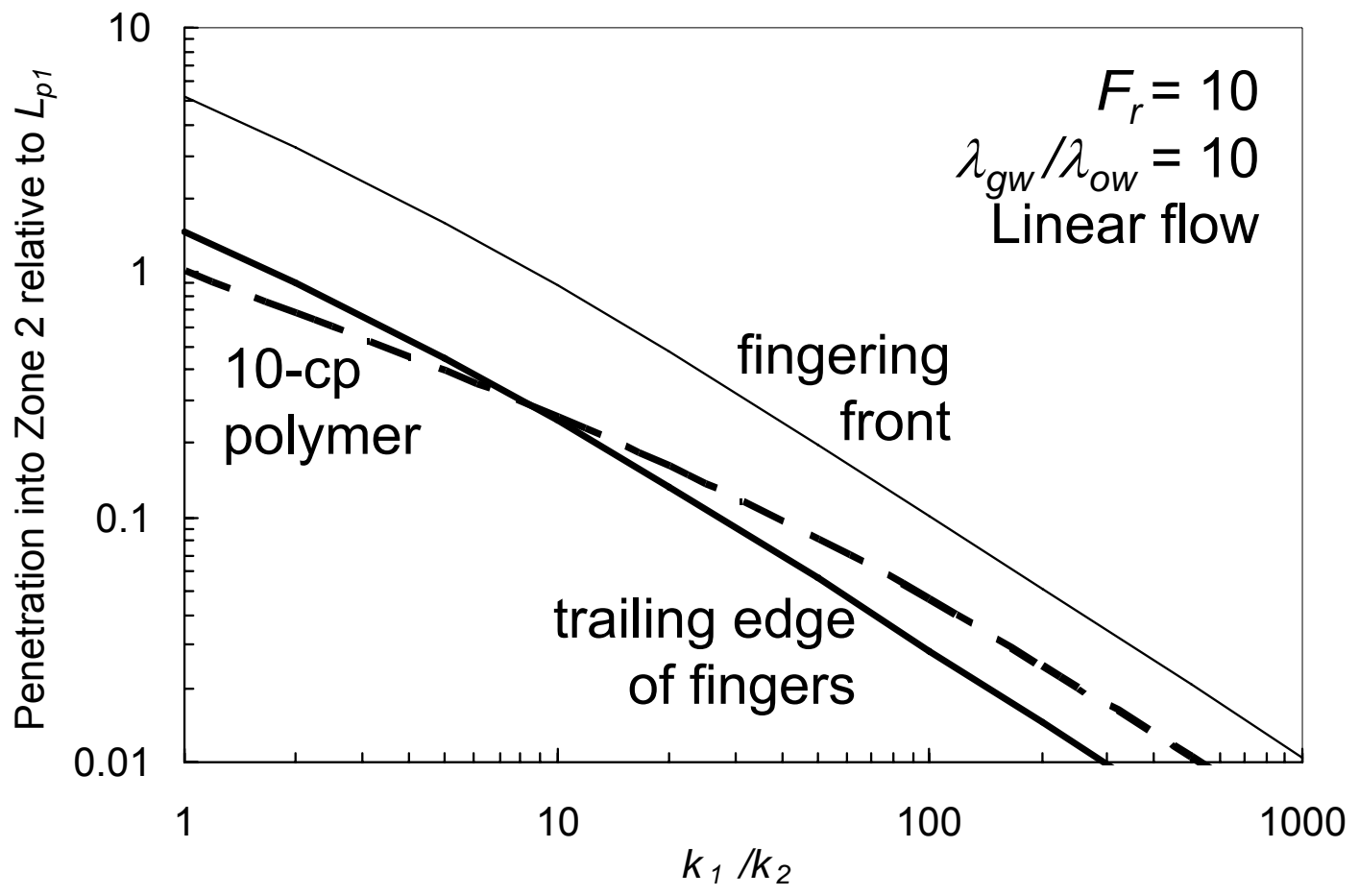

Fig. 68-Fronts in Zone 2 versus permeability contrast. Linear flow.

Radial Flow. Figs. 69-71 show behavior predicted for radial flow. Qualitatively, the trends and observations from these figures were similar to those from the linear-flow cases (Figs. 66-68). However, the effects of variations in mobility ratio, resistance factor, and permeability contrast were less dramatic for radial flow than for linear flow. 


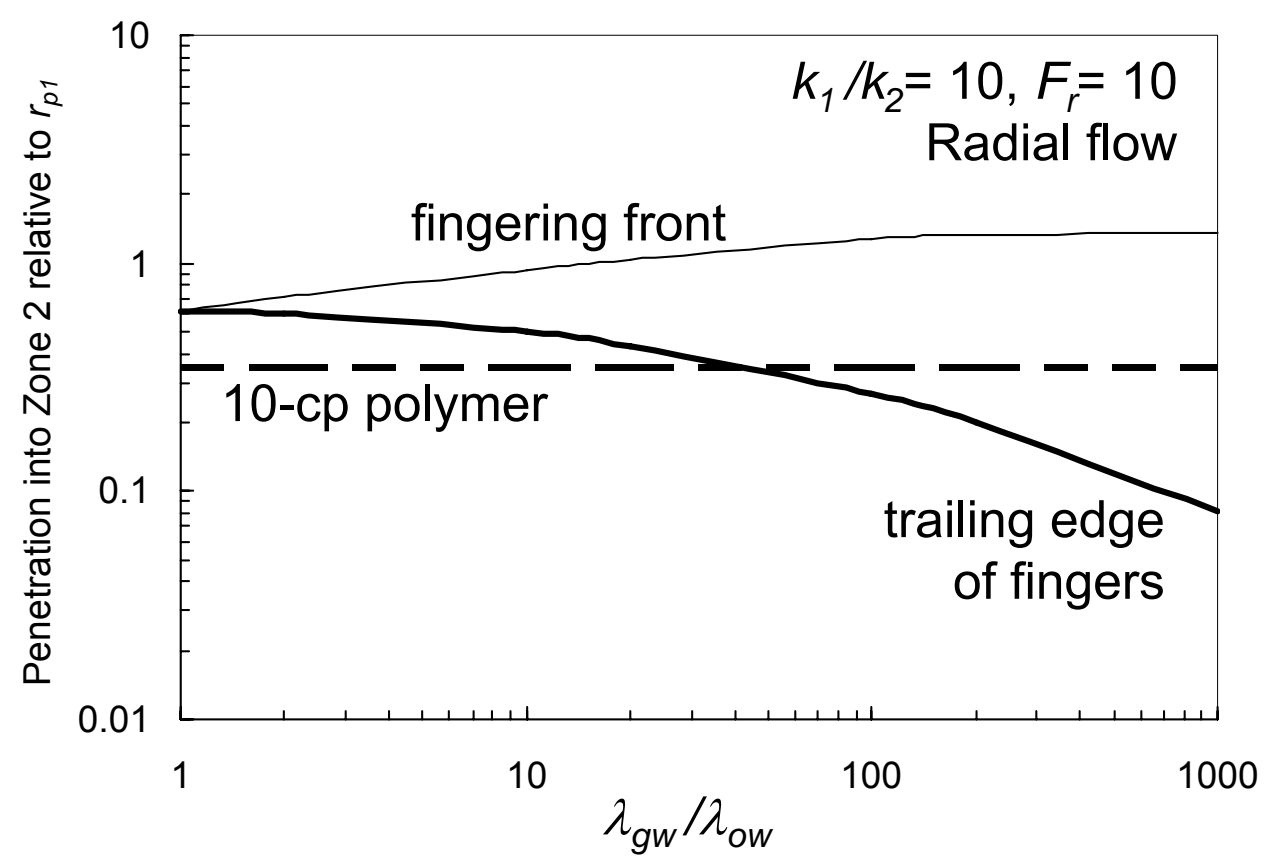

Fig. 69-Fronts in Zone 2 versus gas-water mobility. Radial flow.

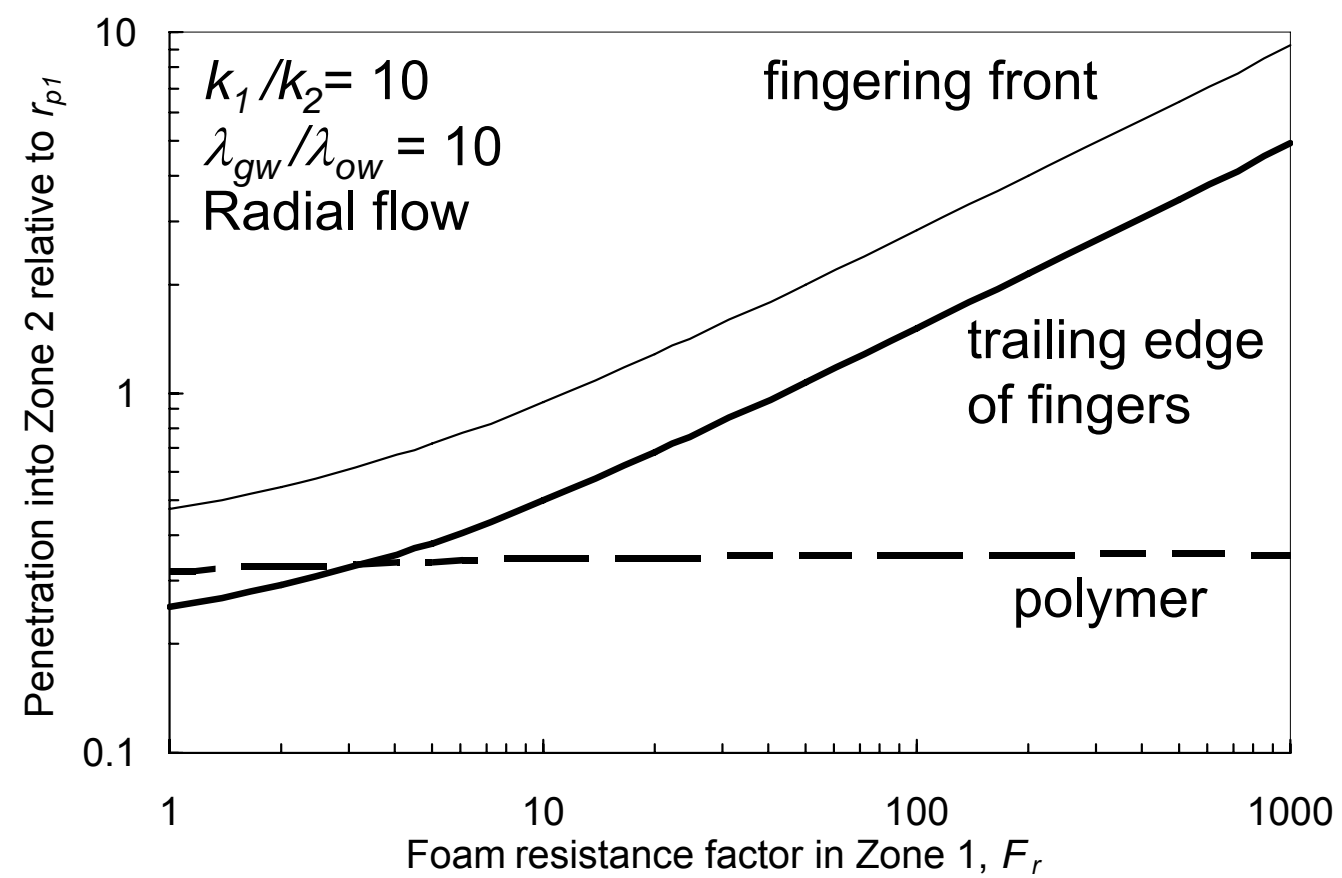

Fig. 70-Fronts in Zone 2 versus resistance factor in Zone 1. Radial flow. 


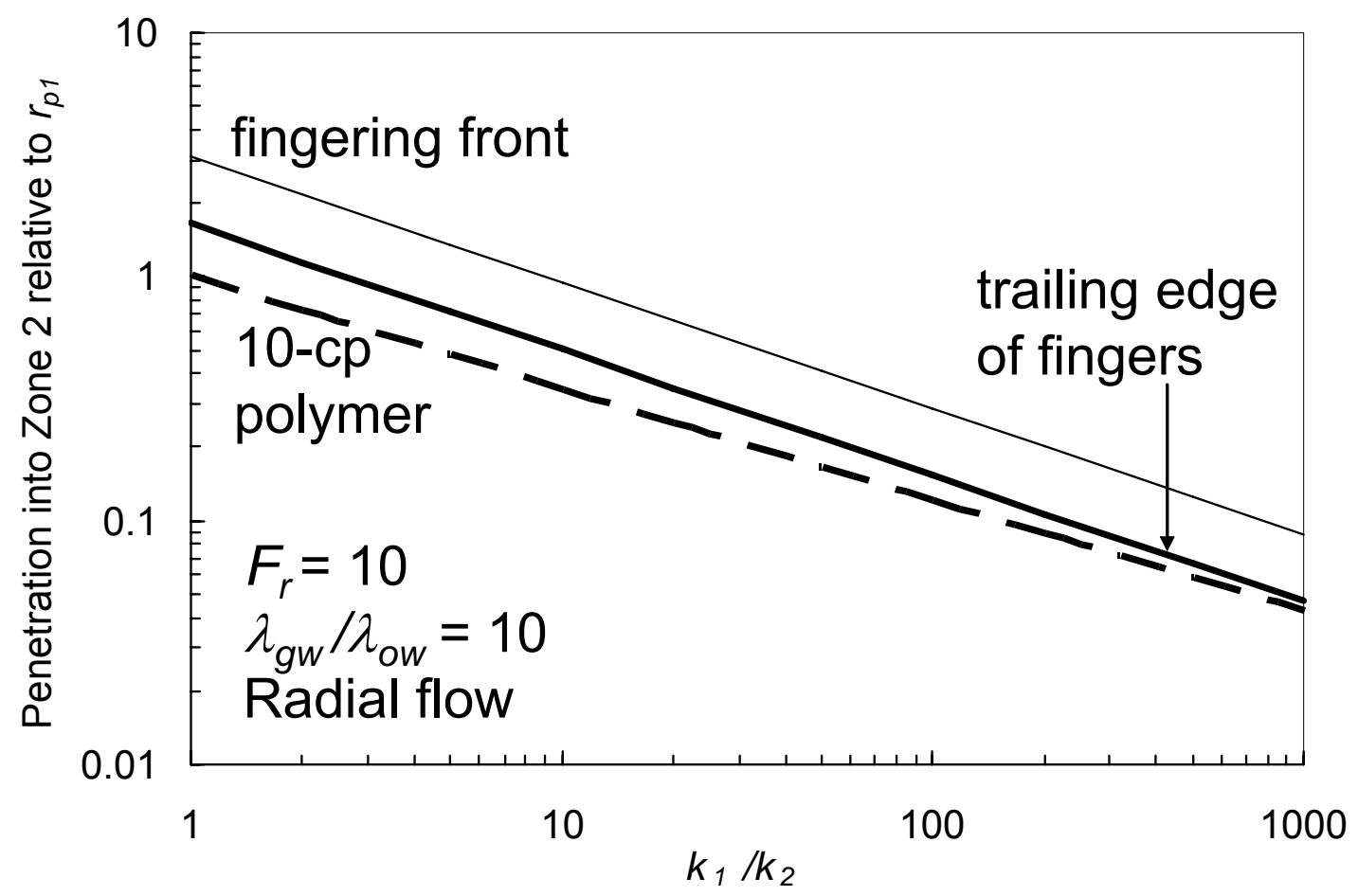

Fig. 71-Fronts in Zone 2 versus permeability contrast. Radial flow.

Examination of Figs. 66-71 indicates that achieving a high resistance factor in Zone 1 (while preventing foam formation in Zone 2) is the key to making foam flooding superior to polymer flooding. In Figs. 72 and 73, we plot penetration for a gas/water/surfactant formulation in Zone 2 versus permeability contrast and mobility ratio for cases where the product, $F_{r} k_{1} / k_{2}$, is held constant at unity. The flat curve profiles in these figures emphasize that a sufficiently large foam resistance factor in Zone 1 can overcome detrimental sweep associated with a large permeability contrast. As a reminder, with viscous polymer solutions, the distance of penetration into Zone 2 is limited by the square root of the permeability contrast ${ }^{6,17,129,164}$ (see Figs. 55 and 56).

\section{Conclusions}

Use of foams could provide superior vertical sweep compared to polymer solutions if (1) foam forms in the high-permeability zone(s) but not in the low-permeability zone(s), (2) no crossflow occurs between the high- and low-permeability zones, AND (3) the foam resistance factor in the high-permeability zone(s) is sufficiently high to overcome the permeability contrast and the unfavorable mobility ratio between the gas bank and the oil/water bank in the less-permeable zones. Foams will generally not be superior to polymers under other circumstances (unless gravity effects provide a fortuitous benefit ${ }^{167}$ ).

Other limitations associated with foam use must also be recognized, ${ }^{17,161-163,166,167}$ including (1) challenges with formulating foams for individual reservoirs to meet the above requirements, (2) limitations on foam propagation, especially due to surfactant retention, (3) compression costs associated with foam injection, and (4) limitations on foam stability under reservoir conditions. 


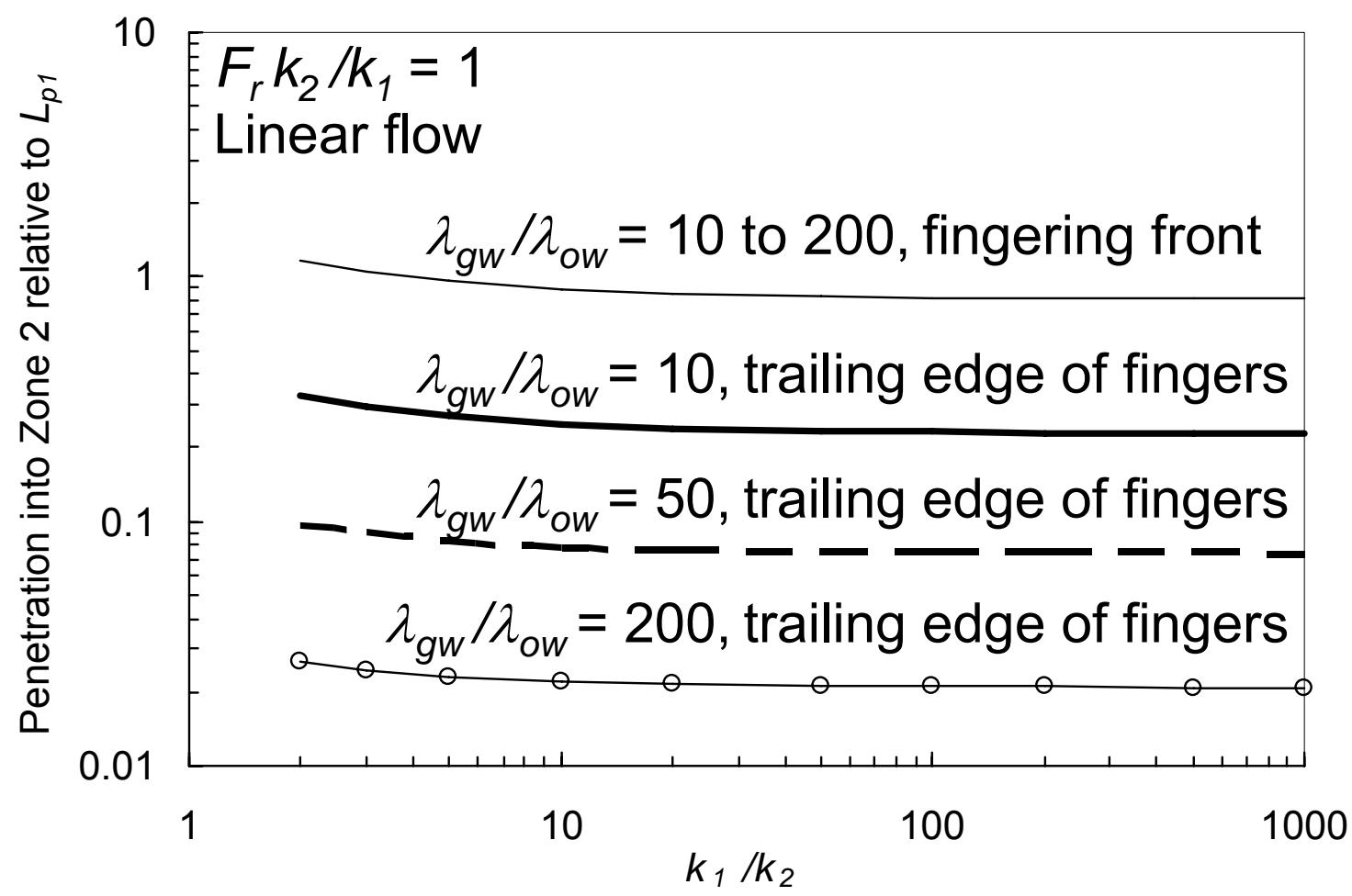

Fig. 72-Fronts in Zone 2 for constant $F_{r} k_{2} / k_{1}$. Linear flow.

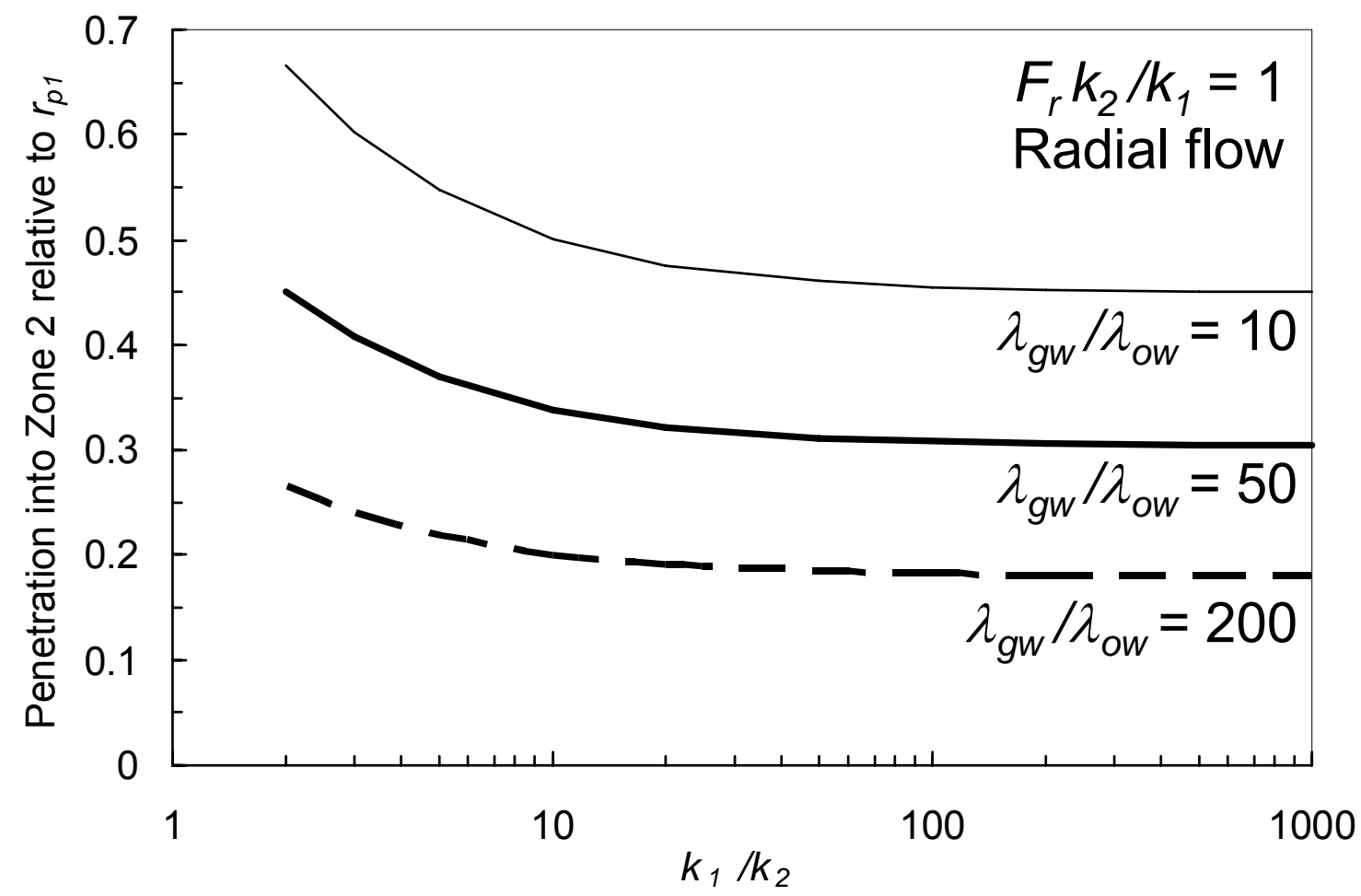

Fig. 73-Trailing edges of the fingering region in Zone 2 for constant $F_{r} k_{2} / k_{1}$. Radial flow. 


\section{HOW IS VERTICAL SWEEP AFFECTED WHEN RESISTANCE FACTORS VARY WITH PERMEABILITY?}

Early work ${ }^{19,20,169-171}$ recognized that high molecular weight partially hydrolyzed polyacrylamides (HPAM) sometimes reduced the mobility $(\lambda$ or $k / \mu)$ of aqueous solutions in porous media by a greater factor than can be rationalized based on the viscosity $(\mu)$ of the solution. The incremental reduction in mobility was attributed to reduction in permeability $(k)$, caused by adsorption or mechanical entrapment of the high molecular weight polymersespecially from the largest polymers in the molecular weight distribution for a given polymer. In the 1960 s and 1970 s, this effect was touted to be of great benefit ${ }^{19,169}$ for polymer floods (simply because the polymer appeared to provide significantly more apparent viscosity in porous media than expected from normal viscosity measurements). However, during the 1980s, at least some research labs (e.g., Exxon Production Research Company) recognized that unexpectedly high mobility reductions from HPAM solutions were either (1) often not achievable in practical field applications or (2) not necessarily of benefit. They were often not achievable in field applications because normal field handling and flow through an injection sand face at high velocities mechanically degraded the large molecules that were responsible for the permeability reduction. ${ }^{132,172}$ Also, the largest molecules were expected to be preferentially retained (i.e., by mechanical entrapment in pores) and stripped from the polymer solution before penetrating deep into the formation.

This chapter addresses the second concern that was raised: Even if high permeability reductions could be achieved, would this effect actually be of benefit? More specifically, the concern focuses on how the mobility reduction varies with permeability of porous media. For adsorbed polymers and suspensions of gel particles, resistance factors $\left(F_{r}\right.$, apparent viscosities in porous media relative to brine) and residual resistance factors ( $F_{r r}$, permeability reduction values) increase with decreasing permeability. ${ }^{14-16,18-21,169-171}$ In other words, these materials reduce the flow capacity of low-permeability rock by a greater factor than high-permeability rock. Depending on the magnitude of this effect, these polymers and gels can harm injection or production flow profiles in wells, even though the polymer or gelant penetrates significantly farther into the high-permeability rock. $7,13,17,129,164$

This chapter examines vertical sweep efficiency as a function of (1) permeability contrast, (2) presence or absence of crossflow, and (3) ratio of resistance factors in neighboring zones. Cases with no crossflow are considered first, followed by cases with crossflow.

\section{Cases with No Crossflow}

As in Chapter 7, our discussion will consider a two-layer reservoir. (The concepts presented here can easily be extended to more complex layering.) The most permeable layer will be called "Zone 1", while the second, less-permeable layer will be called "Zone 2." For a fluid injected to a distance, $L_{p 1}$ (for linear flow) or $r_{p 1}$ (for radial flow), in Zone 1, we are interested in the distance of fluid penetration, $L_{p 2}$ (for linear flow) or $r_{p 2}$ (for radial flow), in Zone 2. The vertical flow profile or vertical sweep efficiency can be assessed using the quantities, $L_{p 2} / L_{p 1}$ for linear flow or $r_{p 2} / r_{p 1}$ for radial flow. When a polymer solution is injected, we are especially interested in how these quantities compare with the permeability contrast between the layers, $k_{2} / k_{1}$. 
Same Resistance Factor in Both Zones. Chapter 7 (especially Figs. 54-56) illustrated the relative penetration of solutions into a two-layer reservoir where an impermeable barrier separated the zones and resistance factors for the injected solution was the same in both zones. If flow was linear (e.g., leakoff into two zones that were cut by a vertical fracture) and if the injected fluid had the same mobility as the fluid being displaced (in both zones), then the distance of injectant penetration should be proportional to the permeability/porosity ratio $(k / \phi)$ for a given zone (Fig. 54). ${ }^{7,13,17,129,164}$ If the injected fluid was quite viscous and the effective viscosity (resistance factor) was the same in both zones, the distance of penetration should be roughly proportional to the square root of the permeability/porosity ratio for a given zone (Fig. $55)^{7,13,17,129,164}$

For the case of radial flow with barriers to crossflow and where resistance factors were the same in both zones, $7,13,17,129,164$ the distance of penetration was roughly proportional to the square root of the permeability/porosity ratio for a given zone (Fig. 56). ${ }^{7,13,17,129,164}$

Linear Flow, Different Resistance Factor in the Zones. For the calculations illustrated in the above figures, the resistance factors (effective viscosities) in Zones 1 and 2 were assumed to be equal. The spreadsheets mentioned in Chapter 7 are capable of performing calculations with different resistance factors in different zones. We used these spreadsheets to generate Fig. 74 for linear flow. The $x$-axis plots the resistance factor in Zone $1\left(F_{r l}\right)$. We are interested in how high the resistance factor can be in Zone $2\left(F_{r 2}\right)$ without impairing sweep efficiency. For this criterion to be met, the ratio, $L_{p 2} / L_{p 1}$, must be greater than the zonal permeability contrast, $k_{2} / k_{1}$. Consequently, the $y$-axis plots the maximum allowable ratio, $F_{r 2} / F_{r 1}$, that meets this criterion. These calculations were made for several permeability ratios, $k_{1} / k_{2}$, between 2 and 20. Fig. 74 shows that for $F_{r 1}$ values greater than 10, the maximum allowable ratio of $F_{r 2} / F_{r 1}$ was about the same as the permeability ratio, $k_{1} / k_{2}$. Thus, linear flow applications can be reasonably forgiving if the permeability contrast and the polymer solution resistance factors are sufficiently large.

Radial Flow, Different Resistance Factor in the Zones. Similar calculations were performed for radial flow, and the results are shown in Fig. 75. These calculations reveal that radial flow is much less forgiving to high values of $F_{r 2} / F_{r 1}$. Even for high permeability contrasts (e.g., $k_{1} / k_{2}=20$ ), the maximum allowable $F_{r 2} / F_{r 1}$ values were less than 1.4 .

Comparison with Laboratory Data. Vela et al. ${ }^{18}$ and Hirasaki and Pope ${ }^{20}$ reported resistance factors obtained in cores with a range of permeability. Both groups used Dow Pusher $700^{\mathrm{TM}}$ HPAM, which had a molecular weight of $5-5.5 \times 10^{6}$ daltons and a degree of hydrolysis of about $30 \%$. Vela et al. used $0.06 \%$ HPAM in $13.3 \%$ total dissolved solids brine at $150^{\circ} \mathrm{F}$. Their reservoir cores were all polymer flooded at residual oil saturation $\left(S_{o r}\right)$ using cores with prepolymer $k_{w}$ at $S_{o r}$ ranging from 12 to $453 \mathrm{md}$. Measured resistance factors from Vela et al. are plotted in Fig. 76. The thin dashed line in Fig. 76 plots the maximum acceptable behavior of resistance factors for linear flow cases, while the thick dashed line plots the maximum acceptable behavior of resistance factors for radial flow cases. In these plots, $F_{r l}$ refers to the measured resistance factor in the 453-md rock, while $F_{r 2}$ refers to the maximum acceptable resistance factor in a given less-permeable rock or zone. For the data provided, the measured behavior of resistance factors was barely acceptable for linear flow, and definitely unacceptable for radial 
flow. A logical remedy for this situation would be to choose a polymer with a lower molecular weight, so resistance factors do not increase so much with decreasing permeability.

Hirasaki and Pope used $0.05 \%$ HPAM in an unspecified brine at $95^{\circ} \mathrm{F}$. Their sandstone cores had no oil saturation $\left(S_{o r}\right)$ and ranged in permeability from 22 to $359 \mathrm{md}$. Their measured resistance factors are plotted in Fig. 77. Again, the thin dashed line plots the maximum acceptable behavior of resistance factors for linear flow cases, while the thick dashed line plots the maximum acceptable behavior of resistance factors for radial flow cases. In Fig. 77, $F_{r 1}$ refers to the measured resistance factor in the 359-md rock, while $F_{r 2}$ refers to the maximum acceptable resistance factor in a given less-permeable rock or zone. For the data provided, the measured behavior of resistance factors was acceptable for both linear and radial flow for rock permeabilities above $100 \mathrm{md}$. For rock with permeability below $100 \mathrm{md}$, the behavior was still acceptable for linear flow, but not for radial flow. Comparing Figs. 76 and 77 suggests that whether or not a given polymer will be acceptable in a given reservoir may depend on a number of factors, including rock permeabilities, water salinity, presence of residual oil, reservoir temperature, and possibly other factors (such as clay content, pore structure, and degree of mechanical degradation before entering the rock).

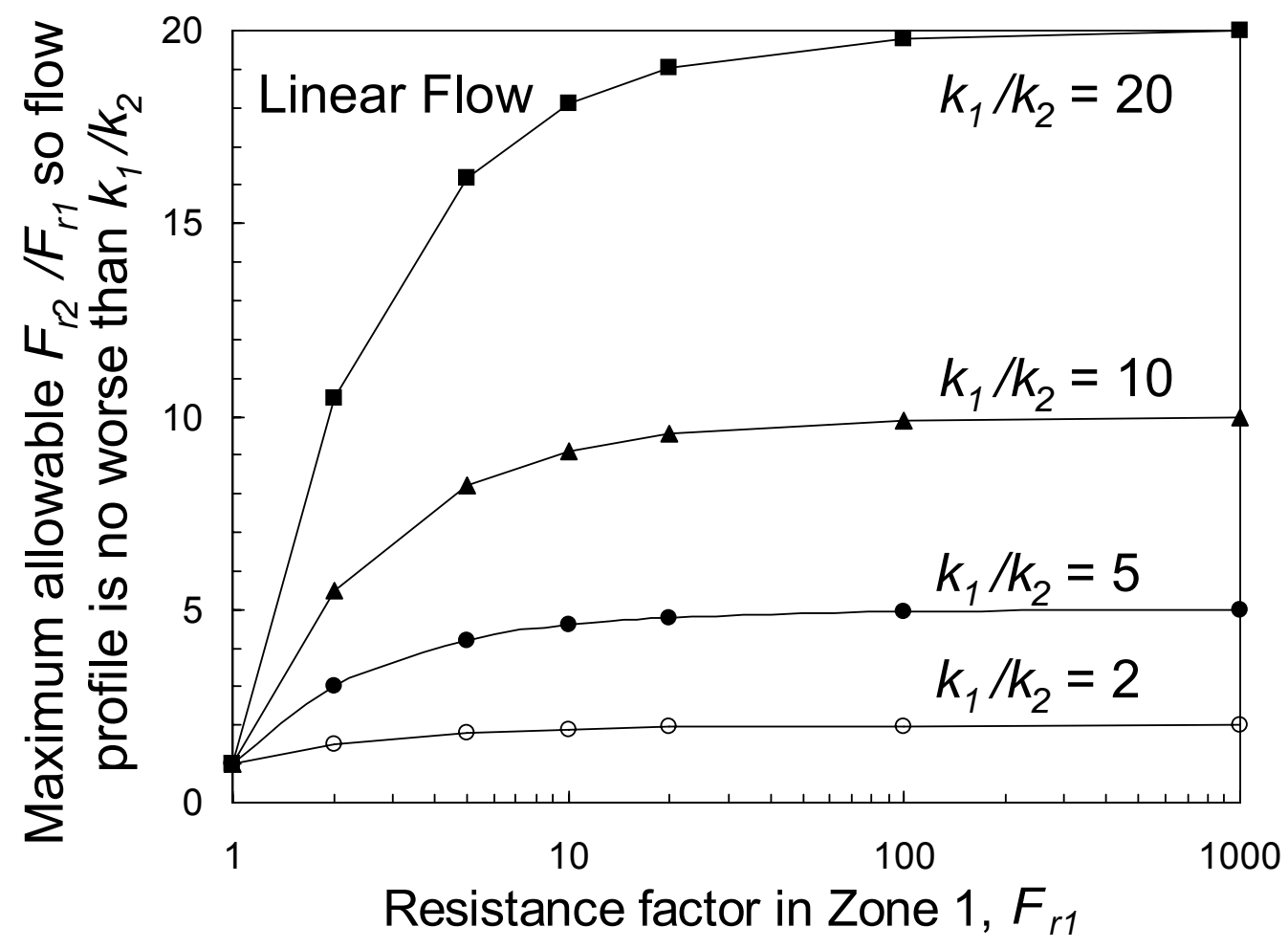

Fig. 74-Maximum allowable $F_{r 2} / F_{r l}$ for linear flow, no crossflow. 


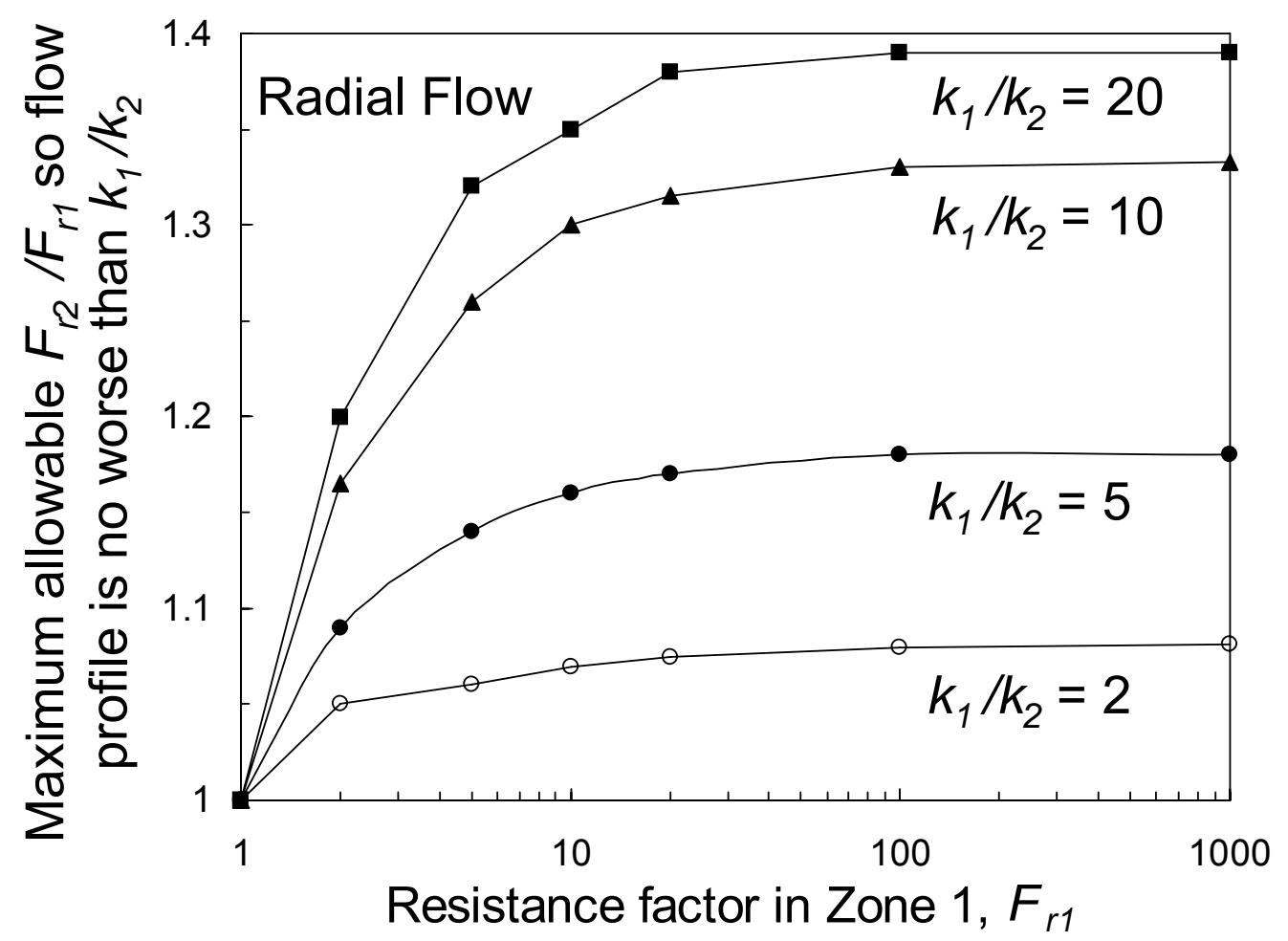

Fig. 75-Maximum allowable $F_{r 2} / F_{r l}$ for radial flow, no crossflow.

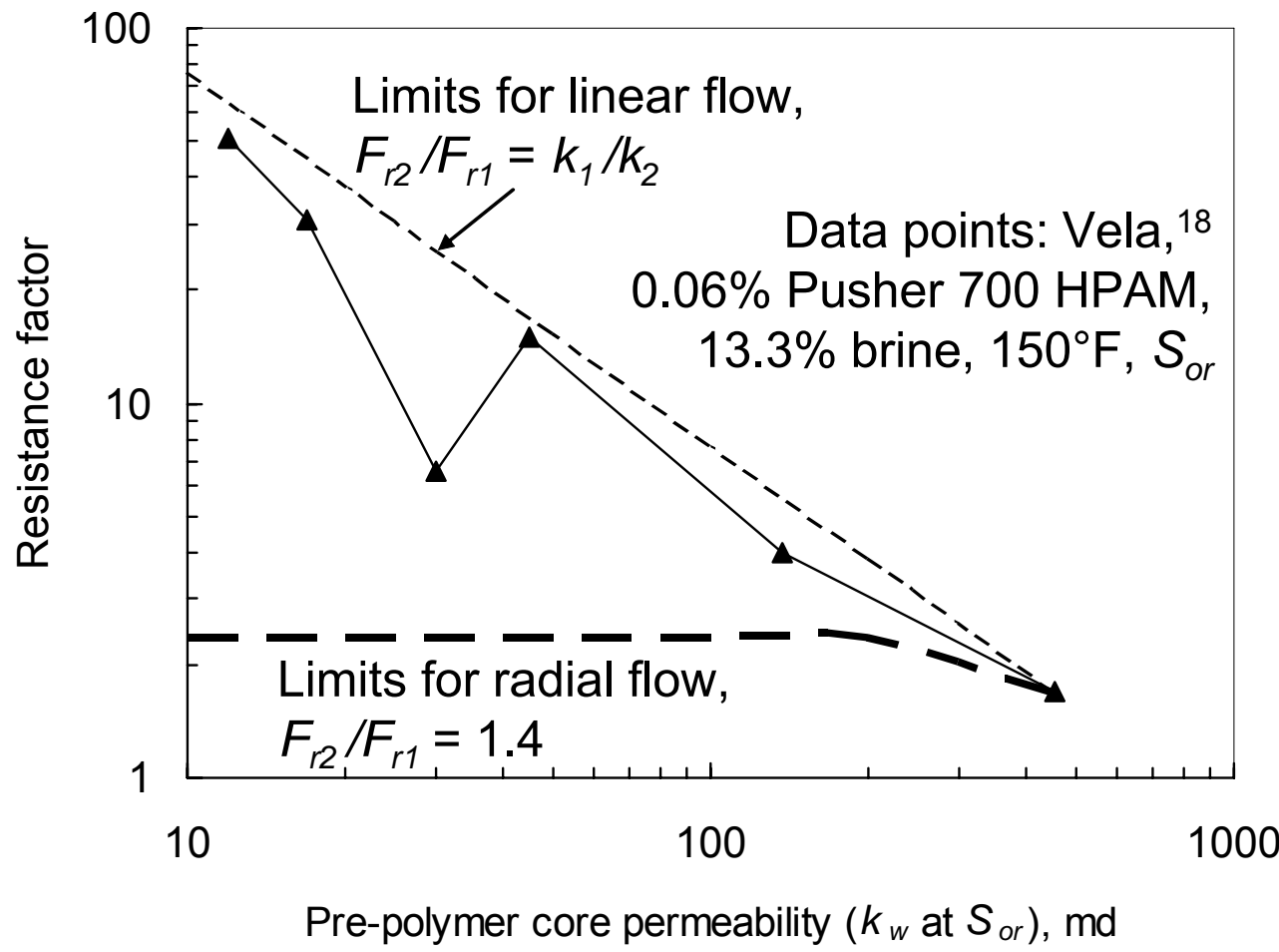

Fig. 76-Data from Vela et al. ${ }^{18}$ compared with maximum allowable $F_{r 2} / F_{r 1}$ values. 


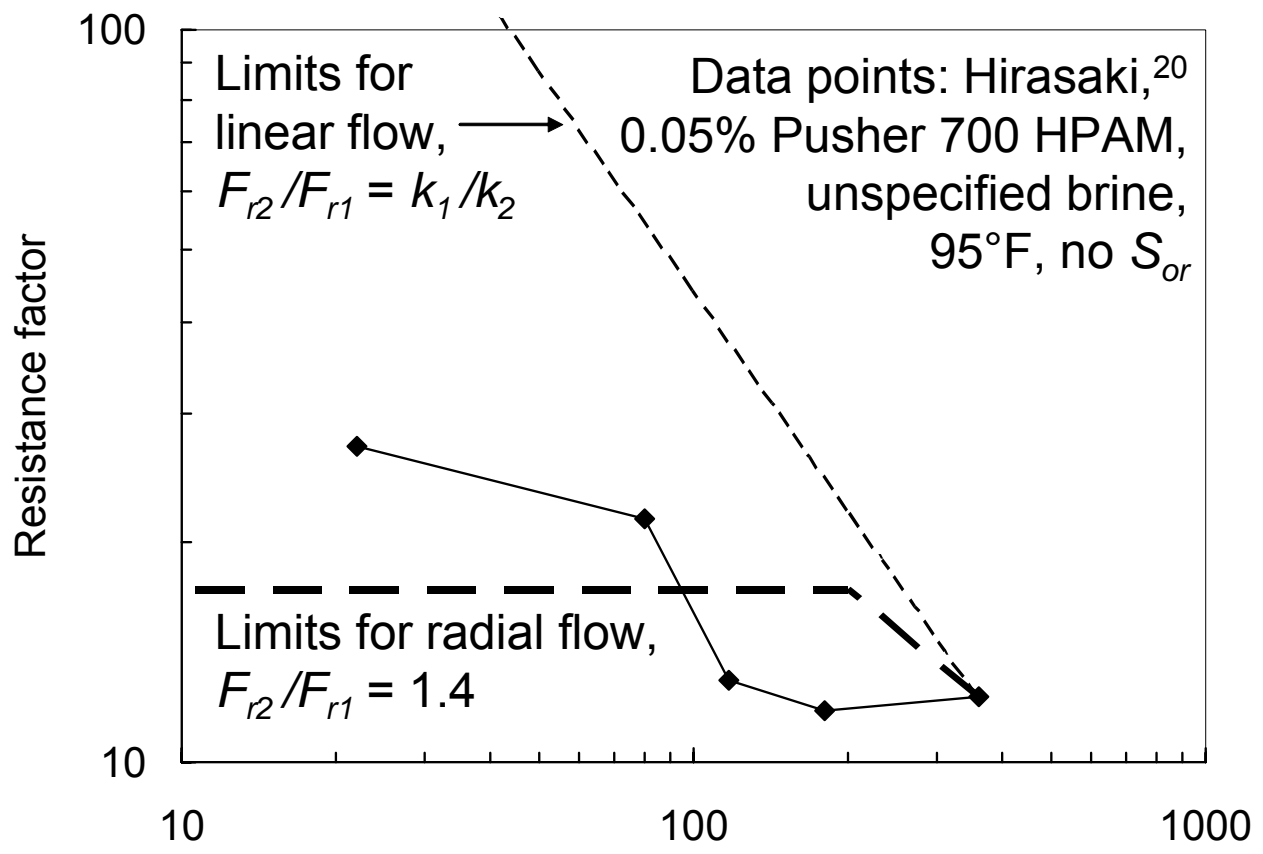

Pre-polymer core permeability $\left(k_{w}\right.$, no $\left.S_{o r}\right)$, md

Fig. 77-Data from Hirasaki and Pope ${ }^{20}$ compared with maximum allowable $F_{r 2} / F_{r l}$ values.

\section{Cases with Crossflow}

The discussion this far focused exclusively on cases with no potential for crossflow between layers (i.e., impermeable barriers exist between zones). In most cases when crossflow can occur, the $F_{r 2} / F_{r 1}$ ratio has little effect on the relative distance of polymer penetration into the various zones. To understand this conclusion, recognize that the distance between wells is usually much greater than the height of any given strata. If a pressure difference (after compensating for gravity) exists between two adjacent communicating zones, crossflow quickly dampens any pressure difference because of zones' close proximity. These observations form the basis of the concept of vertical equilibrium. ${ }^{12,173-175}$ For vertical equilibrium, the pressure gradients in two adjacent zones (with no flow barriers) are the same for any given horizontal position. Put another way, for a given distance from the wellbore (if gravity can be neglected), the pressure is the same in both zones.

Consider a polymer solution flowing through two adjacent zones where crossflow can occur (Fig. 78). Zone 1 (the high-permeability zone) has a permeability of $k_{l}$, a porosity of $\phi_{l}$, and exhibits a polymer resistance factor of $F_{r l}$. Zone 2 (the low-permeability zone) has a permeability of $k_{2}$, a porosity of $\phi_{2}$, and exhibits a polymer resistance factor of $F_{r 2}$. The average movement rates for polymer fronts in the two zones are $v_{1}$ and $v_{2}$. Of course, crossflow may make the polymer front uneven (i.e., not vertical) in Zone 2. So in the simple analysis here, we consider the average front positions. If vertical equilibrium exists, the pressure difference between the polymer fronts will be the same in the two zones. Darcy's law can then be applied to estimate the average front movement rates. For Zone 2, this rate, $v_{2}$, is

$v_{2} \cong \Delta p k_{2} /\left(\mu \phi_{2} L\right)$ 
For Zone 1 , this rate, $v_{l}$, is

$v_{l} \cong \Delta p k_{1} /\left(\mu F_{r l} \phi_{1} L\right)$

The ratio of average front rates is

$v_{2} / v_{1} \cong F_{r l} k_{2} \phi_{1} /\left(k_{1} \phi_{2}\right)$

Consequently, the relative rate of polymer front movement is not sensitive to the resistance factor in Zone 2. Eq. 50 is the same expression that is derived when resistance factors are equal for the two zones.

Figs. 59-61 in Chapter 7 show the approximate front profiles anticipated for several cases with crossflow.

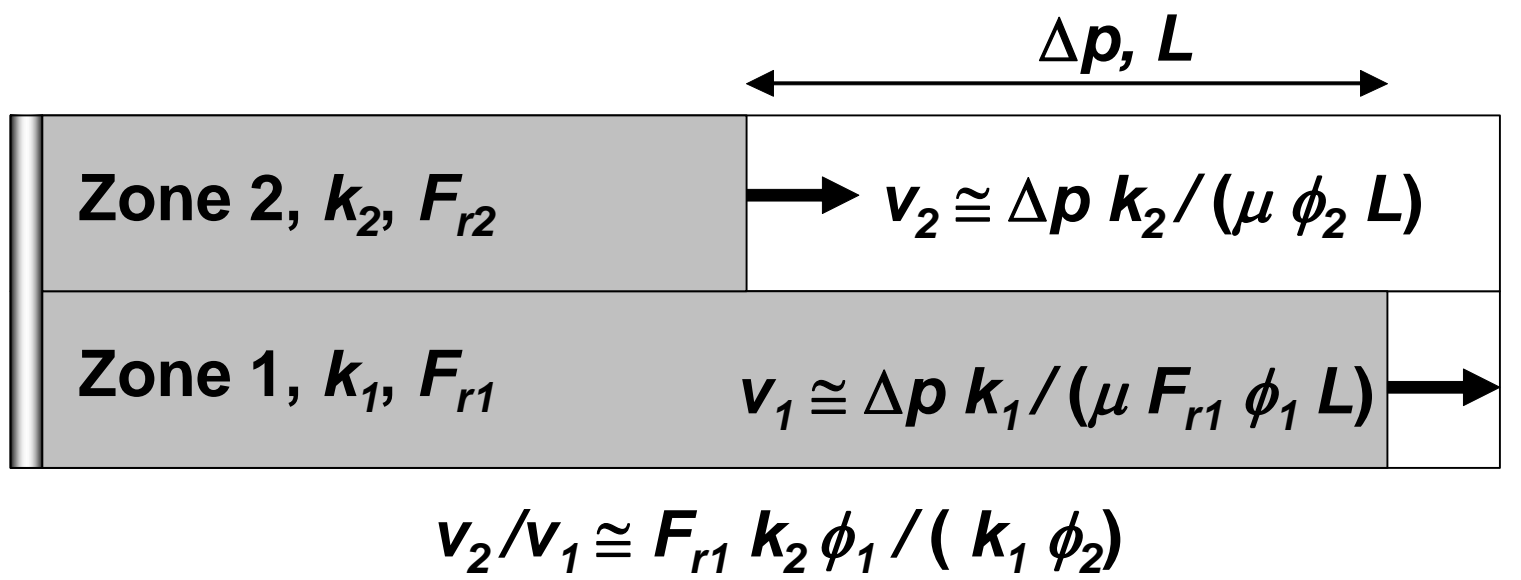

Fig. 78-Understanding front movements for linear flow with crossflow, moderate $F_{r}$ values.

\section{Conclusions}

For applications with linear flow (e.g., fractured wells), the maximum allowable ratio of $F_{r 2} / F_{r 1}$ (so that polymer injection does not harm vertical sweep) is about the same as the permeability ratio, $k_{1} / k_{2}$. Thus, linear flow applications can be reasonably forgiving if the permeability contrast and the polymer solution resistance factors are sufficiently large.

Radial flow is much less forgiving to high values of $F_{r 2} / F_{r 1}$. Even for high permeability contrasts (e.g., $k_{1} / k_{2}=20$ ), the maximum allowable $F_{r 2} / F_{r 1}$ values were less than 1.4.

In most cases when crossflow can occur, the $F_{r 2} / F_{r 1}$ ratio has little effect on the relative distance of polymer penetration into the various zones. 


\section{NOMENCLATURE}

$B=$ parameter in Eq. 6

$C=$ polymer concentration, $\%$

$d p / d l=$ pressure gradient, $\mathrm{psi} / \mathrm{ft}[\mathrm{Pa} / \mathrm{m}]$

$D P R=$ disproportionate permeability reduction

$F=$ force, $\mathrm{N}$

$F_{N}$, normal force, $\mathrm{N}$

$F_{r}=$ resistance factor

$F_{r 1}=$ resistance factor in Zone 1

$F_{r 2}=$ resistance factor in Zone 2

$F_{r r}=$ residual resistance factor (permeability before/after gel placement)

$F_{\text {rro }}=$ residual resistance factor for oil

$F_{r r w}=$ residual resistance factor for water

$F_{\mu}=$ resistance factor in the region of fingering

$G^{\prime}=$ storage modulus, $\mathrm{Pa}$

$G^{\prime \prime}=$ loss modulus, $\mathrm{Pa}$

$h=$ height or gap height or fracture height, $\mathrm{ft}$ [m]

$h_{f}=$ fracture height, $\mathrm{ft}[\mathrm{m}]$

$k=$ consistency index

$k_{f}=$ fracture permeability, darcys $\left[\mu \mathrm{m}^{2}\right]$

$k_{\text {gel }}=$ inherent permeability of gel to water, darcys $\left[\mu \mathrm{m}^{2}\right]$

$k_{m}=$ permeability of matrix or porous rock, darcys $\left[\mu \mathrm{m}^{2}\right]$

$k_{o}=$ permeability to oil, darcys $\left[\mu^{2}\right]$

$k_{r o}=$ relative permeability to oil

$k_{r o}{ }^{o}=$ endpoint relative permeability to oil

$k_{r w}=$ relative permeability to water

$k_{r w}{ }^{o}=$ endpoint relative permeability to water

$k_{v}=$ vertical permeability, darcys $\left[\mu \mathrm{m}^{2}\right]$

$k_{w}=$ permeability to water, darcys $\left[\mu \mathrm{m}^{2}\right]$

$k_{1}=$ permeability for Zone 1 , darcys $\left[\mu^{2}\right]$

$k_{2}=$ permeability for Zone 2, darcys $\left[\mu^{2}\right]$

$L=$ fracture or tube length or distance between wells, $\mathrm{m}$

$L_{e}=$ external drainage distance, $\mathrm{ft}[\mathrm{m}]$

$L_{f}=$ fracture half length, $\mathrm{ft}[\mathrm{m}]$

$L_{p}=$ distance of polymer or gelant leakoff, $\mathrm{ft}[\mathrm{m}]$

$L_{p 1}=$ polymer or foam penetration distance in Zone $1, \mathrm{ft}[\mathrm{m}]$

$L_{p 2}=$ polymer or foam penetration distance in Zone 2, $\mathrm{ft}[\mathrm{m}]$

$N=$ normal stress difference, $\mathrm{Pa}$

$N_{l}=$ larger of two normal stress differences, $\mathrm{Pa}$

$n=$ power index or pore volume exponent in Eq. 6

$n o=$ oil saturation exponent in Eq. 4

$n w=$ water saturation exponent in Eq. 3

$p_{L}=$ outlet pressure, $\mathrm{Pa}$

$p_{0}=$ inlet pressure, $\mathrm{Pa}$

$\Delta p=$ pressure drop, psi $[\mathrm{Pa}]$ 


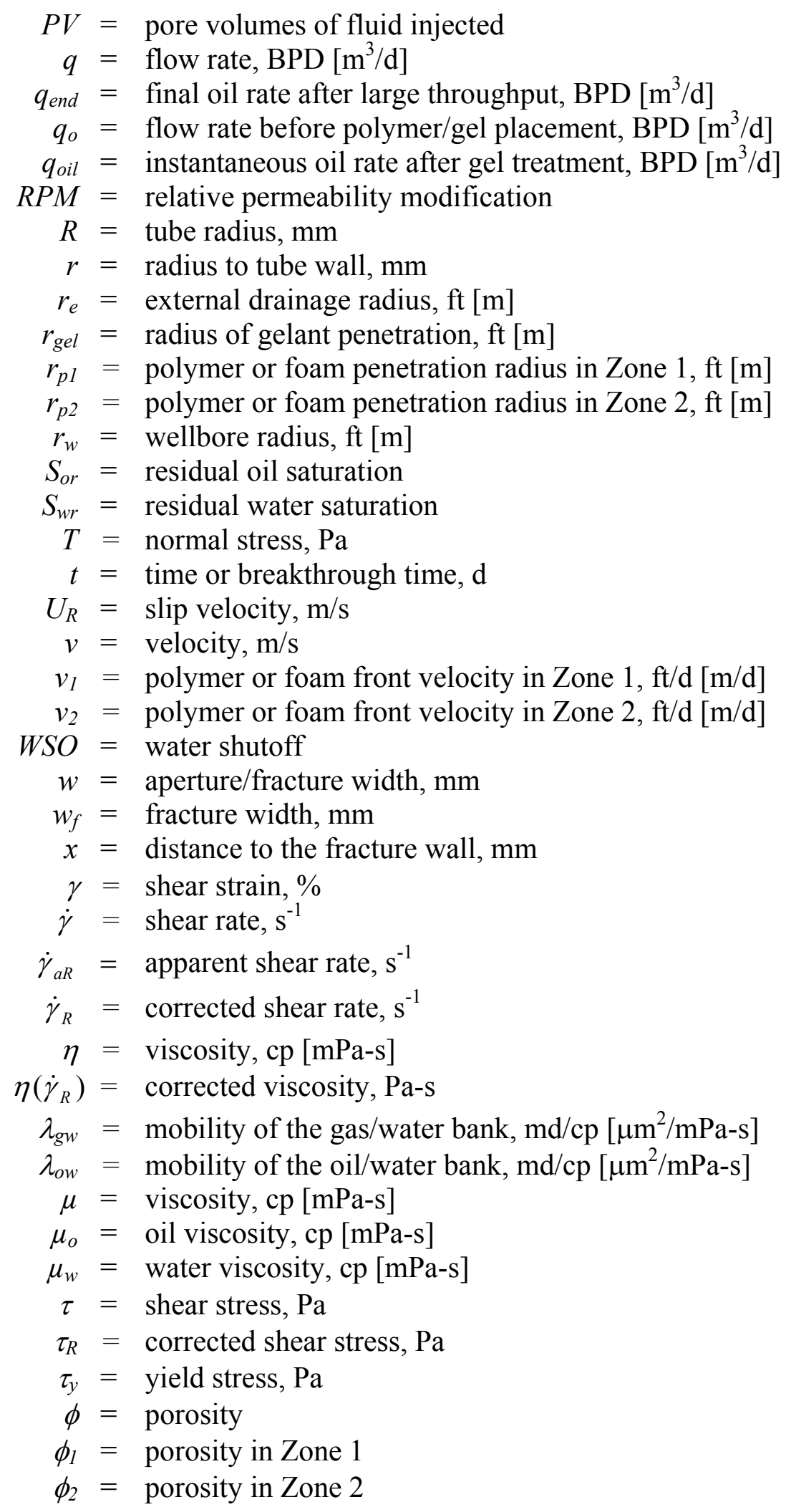




\section{REFERENCES}

1. $\quad$ Liang, J., Sun, H., Seright, R.S.: "Why Do Gels Reduce Water Permeability More Than Oil Permeability?," SPERE, 10(4), (Nov. 1995) 282-286.

2. Seright, R.S.: "Reduction of Gas and Water Permeabilities Using Gels," SPEPF, 10(2), (May 1995) 103-108.

3. Zaitoun, A. and Bertin, H.: "Two-Phase Flow Property Modifications by Polymer Adsorption," paper SPE 39631 presented at the 1996 SPE/DOE Improved Oil Recovery Symposium, Tulsa, OK, April 19-22.

4. Al-Sharji, H.H. et al.: "Pore-Scale Study of the Flow of Oil and Water through Polymer Gels," paper SPE 56738 presented at the 1999 SPE Annual Technical Conference and Exhibition, Houston, TX, Oct. 3-6.

5. Willhite, G.P. et al:: "Mechanisms Causing Disproportionate Permeability in Porous Media Treated With Chromium Acetate/HPAAM Gels," SPEJ, 7(1), (March 2002) 100-108.

6. Liang, J., Lee, R.L., and Seright, R.S.: "Placement of Gels in Production Wells," SPEPF, 8(1), (Nov. 1993) 276-284; Transactions AIME 295.

7. Seright, R.S., Liang, J., and Seldal, M.: "Sizing Gelant Treatments in Hydraulically Fractured Production Wells," SPEPF, 13(4), (Nov. 1998) 223-229.

8. Seright, R.S., Liang, J., and Sun, H.: "Gel Treatments in Production Wells with Water Coning Problems," In Situ, 17(3), (1993) 243-272.

9. Marin, A., Seright, R., Hernandez, M., Espinoza, M., Mejias, F.: “Connecting Laboratory and Field Results for Gelant Treatments in Naturally Fractured Production Wells," paper SPE 77411 presented at the 2002 SPE Annual Technical Conference and Exhibition, San Antonio, TX, Sept. 29- Oct. 2.

10. Seright, R.S.: "Conformance Improvement Using Gels," first annual technical progress report, Contract No. DE-FC26-01BC15316, U.S. DOE (Sept. 2002) 43-59.

11. Pietrak, M.J. et al.: "Relative Permeability Modifier Treatments on Gulf of Mexico FracPacked and Gravel-Packed Oil and Gas Wells," paper SPE 96945 presented at the 2005 SPE Annual Technical Conference and Exhibition, Dallas, TX, Oct. 9-12.

12. Seright, R.S. and Martin, F.D.: "Impact of Gelation pH, Rock Permeability, and Lithology on the Performance of a Monomer-Based Gel," SPERE, 9(1), (Feb. 1993) 43-50.

13. http://baervan.nmt.edu/randy.

14. Seright, R.S.: "Effect of Rock Permeability on Gel Performance in Fluid-Diversion Applications," In Situ, 17(4), (1993) 363-386.

15. Seright, R.S.: "Impact of Permeability and Lithology on Gel Performance," paper SPE 24190 presented at the 1992 SPE/DOE Symposium on Enhanced Oil Recovery, Tulsa, OK, April 22-24.

16. Rousseau, D. et al.: "Rheology and Transport in Porous Media of New Water Shutoff/Conformance Control Microgels," paper SPE 93254 presented at the 2005 SPE International Symposium on Oilfield Chemistry, Houston, TX, Feb. 2-4.

17. Seright, R.S.: "Placement of Gels to Modify Injection Profiles," paper SPE/DOE 17332 presented at the 1988 SPE/DOE Enhanced Oil Recovery Symposium, Tulsa, OK, April 1720.

18. Vela, S., Peaceman, D.W. and Sandvik, E.I.: "Evaluation of Polymer Flooding in a Layered Reservoir with Crossflow, Retention, and Degradation," SPEJ, 16(2), (April 1976) 82-96.

19. Jennings, R.R., Rogers, J.H., and West, T.J.: "Factors Influencing Mobility Control by Polymer Solutions," JPT, 23(3), (Mar. 1971) 391-401. 
20. Hirasaki, G.J., and Pope, G.A.: "Analysis of Factors Influencing Mobility and Adsorption in the Flow of Polymer Solution Through Porous Media”, SPEJ, 14(4), (Aug. 1974) 337346.

21. Zaitoun, A., and Kohler, N.: Two-Phase Flow Through Porous Media: Effect of an Adsorbed Polymer Layer," paper SPE 18085 presented at the 1988 SPE Annual Technical Conference and Exhibition, Houston, TX, Oct. 2-5.

22. Seright, R.S.: "Clean Up of Oil Zones after a Gel Treatment," SPEPF, 21(2), (May 2006).

23. Seright, R.S: "Mechanism for Gel Propagation through Fractures," paper SPE 55628 presented at the 1999 SPE Rocky Mountain Regional Meeting, Gillette, WY, May 15-18.

24. Seright, R.S.: "Using Chemicals to Optimize Conformance Control in Fractured Reservoirs," first annual report (DOE/BC/15110-2), Contract No. DE-AC26-98BC15110, U.S. DOE (Sept. 1999) 24.

25. Seright, R.S., Prodanovic, M., and Lindquist, W.B.: "X-Ray Computed Microtomography Studies of Fluid Partitioning in Drainage and Imbibition Before and After Gel Placement," SPEJ, 11(2), (June 2006).

26. Seright, R.S, Liang J., Lindquist, W.B., and Dunsmuir, J.H.: "Characterizing Disproportionate Permeability Reduction Using Synchrotron X-Ray Computed Microtomography," SPEREE, 5(5), (Oct. 2002) 355-364.

27. Sandiford, B.B.: "Laboratory and Field Studies of Water Floods Using Polymer Solutions to Increase Oil Recovery," JPT (August 1964) 917-922.

28. White, J.L., Goddard, J.E., and Phillips, H.M.: "Use of Polymers to Control Water Production in Oil Wells," JPT (February 1973) 143-150.

29. Sparlin, D.D.: "An Evaluation of Polyacrylamides for Reducing Water Production," JPT (August 1976) 906-915.

30. Weaver, J.D.: “A New Water-Oil Ratio Improvement Material," paper SPE 7574 presented at the 1978 Annual Fall Technical Conference and Exhibition, Dallas, TX, 1-3 October.

31. VanLandingham, J.V.: "Laboratory \& Field Development of Dispersed Phase Polymer Systems for Water Control," paper SPE 8423 presented at the 1979 Annual Fall Technical Conference and Exhibition, Las Vegas, Nevada, 23-26 September.

32. Schneider, F.N. and Owens, W.W.: "Steady-State Measurements of Relative Permeability for Polymer/Oil Systems," SPEJ (February 1982) 79-86.

33. Kohler, N. et al.: "Weak Gel Formulations for Selective Control of Water Production in High-Permeability and High-Temperature Production Wells," paper SPE 25225 presented at the 1983 International Symposium on Oilfield Chemistry, New Orleans, Louisiana, 2-5 March.

34. Dunlap, D.D., Boles, J.L., and Novotny, R.J.: "Method for Improving Hydrocarbon/Water Ratios in Producing Wells," paper SPE 14822 presented at the 1986 Symposium on Formation Damage Control, Lafayette, Louisiana, 26-27 February.

35. Dovan, H.T. and Hutchins, R.D.: "New Polymer Technology for Water Control in Gas Wells," SPEPF (November 1994) 280-286.

36. Stanley, F.O. et al.: "Amphoteric Polymer Improves Hydrocarbon/Water Ratios in Producing Wells - An Indonesian Case Study,” SPEPF (August 1997) 181-186.

37. Faber, M.J. et al.: "Water Shut-Off Field Experience With a Relative Modification System in the Marmul Field (Oman)," paper SPE 39633 presented at the 1998 Improved Oil Recovery Symposium, Tulsa, OK, 19-22 April. 
38. Eoff, L et al.: "Structure and Process Optimization for the Use of a Polymeric RelativePermeability Modifier in Conformance Control," SPEJ (June 2003) 92-98.

39. Ligthelm, D.J.: "Water Shut Off in Gas Wells: Is There Scope for a Chemical Treatment?" paper SPE 68978 presented at the 2001 European Formation Damage Conference, The Hague, The Netherlands, 21-22 May.

40. Morgan, J. et al.: "Development and Deployment of a "Bullheadable" Chemical System for Selective Water Shut Off Leaving Oil/Gas Production Unharmed," paper SPE 78540 presented at the $200210^{\text {th }}$ Abu Dhabi International Petroleum Exhibition and Conference.

41. Di Lullo, G. and Rae, P.: "New Insights into Water Control - A Review of the State of the Art," paper SPE 77963 presented at the 2002 Asia Pacific Oil and Gas Conference and Exhibition, Melbourne, Australia, 8-10 October.

42. Eoff, L. et al:: "Development of Hydrophobically Modified Water-Soluble Polymer as a Selective Bullhead System for Water-Production Problems," paper SPE 80206 presented at the 2003 International Symposium on Oilfield Chemistry, Houston, TX, 5-7 February.

43. Kume, N.: "An Engineering Approach to Predicting Post-Treatment Well Performance Using Selective Chemical Water Shut-Off Techniques: An RPM Example," paper SPE 84513 presented at the 2003 SPE Annual Technical Conference and Exhibition, Denver, CO, 5-8 October.

44. Seright, R.S.: “Optimizing Disproportionate Permeability Reduction,” paper SPE 99443 presented at the 2006 SPE/DOE Symposium on Improved Oil Recovery, Tulsa, OK, 22-26 April.

45. Sydansk, R.D. et al.: "Characterization of Partially Formed Polymer Gels for Application to Fractured Production Wells for Water-Shutoff Purposes," SPEPF (August 2005) 240249.

46. Dawe, R.A. and Zhang, Y.: "Mechanistic Study of the Selective Action of Oil and Water Penetrating into a Gel Emplaced in a Porous Medium, J. of Pet. Sci. \& Eng. 12 (1994) 113125.

47. Liang, J-T. and Seright, R.S.: "Further Investigations of Why Gels Reduce Water Permeability," SPE Production \& Facilities (Nov. 1997) 225-230.

48. Barreau, P. et al.: "Water Control in Producing Wells: Influence of an Adsorbed-Polymer Layer on Relative Permeabilities and Capillary Pressure," SPERE (November 1997) 234239.

49. Thompson, K.E. and Fogler, H.S.: "Pore-Level Mechanisms for Altering Multiphase Permeability with Gels," SPEJ (1997) 350-362.

50. Nilsson, S., Stavland, A., and Jonsbraten, H.C.: "Mechanistic Study on Disproportionate Permeability Reduction," paper SPE 39635 presented at the 1998 Improve Oil Recovery Symposium, Tulsa, OK, 19-22 April.

51. Memmella, A. et al.: "Pore-Scale Mechanism for Selective Permeability Reduction by Polymer Injection," paper SPE 39634 presented at the 1998 Improved Recovery Symposium, Tulsa, OK, 19-22 April.

52. Zitha, P.L.J., Vermolen, F.J., and Bruining, H.: "Modification of Two Phase Flow Properties by Adsorbed Polymers or Gels," paper SPE 54737 presented at the 1999 European Formation Damage Conference, The Hague, The Netherlands, 31 May - 1 June.

53. Al-Sharji, H.H. et al.: "Disproportionate Permeability Reduction Due to Polymer Adsorption Entanglement," paper SPE 68972 presented at the 1999 European Formation Damage Conference, The Hague, The Netherlands, 21-22 May. 
54. Elmkies, P. et al.: "Further Investigations on Two-Phase Flow Property Modification by Polymers: Wettability Effects," paper SPE 64986 presented at the 2001 International Symposium on Oilfield Chemistry, Houston, TX, 13-16 February.

55. Stavland, A. and Nilsson, S.: "Segregated Flow Is the Governing Mechanism of Disproportionate Permeability Reduction in Water and Gas Shutoff," paper SPE 71510 presented at the 2001 SPE Annual Technical Conference and Exhibition, New Orleans, Louisiana, 30 September - 3 October.

56. Grattoni, C.A., Jing, X.D., and Zimmerman, R.W.: "Disproportionate Permeability Reduction When a Silicate Gel Is Formed In-Situ to Control Water Production," paper SPE 69534 presented at the 2001 Latin American and Caribbean Petroleum Engineering Conference, Buenos Aries, Argentina, 25-28 March.

57. Gludicellie, C.B and Truchetet, R.A.: "Prediction of the Oil Rate Increment With Water Profile Control by Polymer Injection," paper SPE 26608 presented at the 1993 Annual Technical Conference and Exhibition, Houston, TX, 3-6 October.

58. Stavland, A. et al:: "Disproportionate Permeability Reduction Is Not a Panacea," SPEREE (August 1998) 359-366.

59. Zaitoun, A. et al.: "Water Shutoff by Relative Permeability Modifiers: Lessons from Several Field Applications," paper SPE 56740 presented at the 1999 Annual Technical Conference and Exhibition, Houston, TX, 3-6 October.

60. Mennella, A. et al.: "Candidate and Chemical Selection Guidelines for Relative Permeability Modification (RPM) Treatments," SPEPF (August 2001) 181-188.

61. Botermans, C.W., Van Batenburg, D.W., and Bruining, J.: "Relative Permeability Modifiers: Myth or Reality?" paper SPE 68973 presented at the 2001 European Formation Damage Conference, The Hague, The Netherlands, 21-22 May.

62. Kabir, A.H.: "Chemical Water \& Gas Shutoff Technology - An Overview," paper SPE 72119 presented at the 2001 Asia Pacific Improved Oil Recovery Conference, Kuala Lumpur, Malaysia, 8-9 October.

63. Kalfayan, L.J. and Dawson, J.C.: "Successful Implementation of Resurgent Relative Permeability Modifier (RPM) Technology in Well Treatments Requires Realistic Expectations," paper SPE 90430 presented at the 2004 Annual Technical Meeting and Exhibition, Houston, TX, 26-29 September.

64. Seright, R.S., Lane, R.H., and Sydansk, R.D.: “A Strategy for Attacking Excess Water Production," SPE Production and Facilities (Aug. 2003) 158-169.

65. Al-Dhafeeri, A.M. et al.: "High-Permeability Carbonate Zones (Super-K) in Ghawar Field (Saudi Arabia): Identified, Characterized, and Evaluated for Gel Treatments," paper SPE 97542 presented at the 2005 SPE International Improved Oil Recovery Conference in Asia Pacific, Kuala Lumpur, Malaysia, 5-6 December.

66. Lane, R.H., and Seright, R.S.: "Gel Water Shutoff in Fractured or Faulted Horizontal Wells," paper CIM/SPE 65527 presented at the 2000 SPE/Petroleum Society of CIM International Conference on Horizontal Well Technology held in Calgary, November 6-8.

67. Lane, R.H. and Sanders, G.S: "Water Shutoff Through Fullbore Placement of Polymer Gel in Faulted and in Hydraulically Fractured Producers of the Prudhoe Bay Field," paper SPE 29475 presented at the 1995 SPE Production Operations Symposium, OK City, OK, April 2-4.

68. Sydansk, R.D. and Southwell, G.P.: "More Than 12 Years' Experience With a Successful Conformance-Control Polymer-Gel Technology," SPEPF (November 2000) 270-278. 
69. Sydansk, R.D. and Moore, P.E.: "Gel Conformance Treatments Increase Oil Production in WY," Oil \& Gas J. (January 1992) 40.

70. Borling, D.C.: "Injection Conformance Control Case Histories Using Gels at the Wertz Field $\mathrm{CO}_{2}$ Tertiary Flood in WY," paper SPE 27825 presented at the 1994 SPE/DOE Symposium on Improved Oil Recovery, Tulsa, OK, 17-20 April.

71. Hild, G.P. and Wackowski, R.K.: "Reservoir Polymer Gel Treatments To Improve Miscible $\mathrm{CO}_{2}$ Flood," SPEREE (April 1999) 196.

72. Seright, R.S.: "An Alternative View of Filter Cake Formation in Fractures Inspired by Cr(III)-Acetate-HPAM Gel Extrusion,” SPEPF (February 2003) 65.

73. Seright, R.S.: “Gel Propagation through Fractures," SPEPF (November 2001) 225.

74. Prud'homme, R.K. et al.: "Rheological Monitoring of the Formation of Polyacrylamide/Cr ${ }^{3+}$ Gels," SPEJ (October 1983) 804.

75. Prud'homme, R.K. and Uhl, J.T.: "Kinetics of Polymer/Metal-Ion Gelation," paper SPE/DOE 12640 presented at the 1984 AIME SPE/DOE Symposium on Enhanced Oil Recovery, Tulsa, OK, 15-18 April.

76. Pearson, D.S. and Graessley, W.W.: "The Structure of Rubber Networks with Multifunctional Junctions," Macromolecules (1978) 11, No. 3, 528.

77. Aslam, S., Vossoughi, S., and Willhite, G.P.: "Viscometric Measurement of Chromium (III)-Polyacrylamide Gels by Weissenberg Rheogoniometer," paper SPE 12639 presented at the 1984 AIME SPE/DOE Symposium on Enhanced Oil Recovery, Tulsa, OK, 15-18 April.

78. Thurston, G.B., Ozon, P.M., and Pope, G.A.: "Viscoelasticity and Gelation of Some Polyacrylamide and Xanthan Gum Solutions," paper No. 62B presented at the American Institute of Chemical Engineers 1985 Spring National Meeting and Petro Expo '85, Houston, TX, 24-28 March.

79. Kakadjian, S., Rauseo, O., and Mejias, F.: "Dynamic Rheology as a Method to Quantify Gel Strength of Water Shutoff Systems," paper SPE 50751 presented at the 1999 International Symposium on Oilfield Chemistry, Houston, TX, 16-19 February.

80. Allain, C. and Salome, L.: "Gelation of Semidilute Polymer Solutions by Ion Complexation: Critical Behavior of the Rheological Properties versus Cross-Link Concentration," Macromolecules (1990) 23, No. 4, 981.

81. Broseta, D. et al.: "Rheological Screening of Low-Molecular-Weight Polyacrylamide/Chromium (III) Acetate Water Shutoff Gels," paper SPE 59319 presented at the 2000 SPE/DOE Symposium on Improved Oil Recovery, Tulsa, OK, 3-5 April.

82. Liu, J., and Seright, R.S.: "Rheology of Gels Used for Conformance Control in Fractures," SPEJ (June 2001) 120.

83. Liu, J.: "Rheological Properties of Gels Used for Conformance Control," Master Degree thesis, New Mexico Institute of Mining and Technology, Socorro, New Mexico (1999), 1029.

84. Bird, R.B., Dai, G.C., and Yarusso, B.J.: "The Rheology and Flow of Viscoplastic Materials," Reviews in Chem. Eng. (1983) 1, No.1, 1.

85. Seright, R.S.: “Conformance Improvement Using Gels," Semi-Annual Technical Progress Report, U.S. DOE Contract No. DE-FC26-01BC15316, Washington, DC (March 2003), 47-58.

86. Macosko, C.W.: Rheology Principles, Measurements, and Applications, WILEY-VCH, New York City (1994), 97-98, 108-140. 
87. Yoshimura, A. and Prud'homme, R.K.: "Wall Slip Corrections for Couette and Parallel Disk Viscometers," J. of Rheology (1988) 32, No.1, 53.

88. Yoshimura, A. and Prud'homme, R.K.: "Viscosity Measurements in the Presence of Wall Slip in Capillary, Couette, and Parallel-Disk Geometries," SPERE (May 1988) 735.

89. Ganguly, G. et al.: "The Effect of Fluid Leakoff on Gel Placement and Gel Stability in Fractures," SPEJ (September 2002) 309.

90. Taber, J.J., Martin, F.D., and Seright, R.S.: "EOR Screening Criteria Revisited-Part 1: Introduction to Screening Criteria and Enhanced Recovery Field Projects," SPERE (August 1997) 189.

91. Bird, R.B., Stewart, W.E., and Lightfoot, E.N.: Transport Phenomena, John Wiley \& Sons, New York (1976) 42-46, 62-63.

92. Seright, R.S.: "Use of Preformed Gels for Conformance Control in Fractured Systems," SPEPF (February 1997) 59.

93. Seright, R.S.: "Polymer Gel Dehydration during Extrusion through Fractures," SPEPF (May 1999) 110.

94. Broseta, D., Marquer, O., and Zaitoun, A.: "Shear Effects on Polyacrylamide / Chromium (III) Acetate Gelation," paper SPE 50750 presented at the 1999 SPE International Symposium on Oilfield Chemistry, Houston, TX, 16-19 February.

95. Wang, C.Y., Li, Q.Z., Zheng, J.M., and Guo, C.T.: "Die-swell Measurement for the Evaluation of Elasticity of Mesophase Pitches," Carbon (1998) 36, No.12, 1861.

96. Bigg, D.M.: "Rheology and Wire Coating of High Atomic Number Metal-low Density Polyethylene Composites," Polymer Eng. Sci. (1977) 17, No.10, 745.

97. Liang, J.Z.: "Predictions of Primary Normal Stress Difference from Extrudate Swell of Rubber Compound," Plastics, Rubber and Composites Processing and Applications (1996) 25, No. 5, 257.

98. Magda, J.J., Lee, C-S., Muller, S.J.; and Larson, R.G.: "Rheology, Flow Instabilities, and Shear-induced Diffusion in Polystyrene Solutions," Macromolecules (1993) 26, No. 7, 1696.

99. Lerdwijitjarud, W., Sirivat, A., and Larson, R.G.: "Influence of Dispersed-phase Elasticity on Steady-state Deformation and Breakup of Droplets in Simple Shearing Flow of Immiscible Polymer Blends," J. of Rheology (2004) 48, No. 4, 843.

100. Takahashi, Y. et al: "Experimental Tests of the Scaling Relation for Textured Materials in Mixtures of Two Immiscible Fluids," J. of Rheology (1994) 38, No. 3, 699.

101. Meijer, H.E.H. and Bulters, M.J.H.: "Analogy between the Modeling of Pullout in Solution Spinning and the Prediction of the Vortex Size in Contraction Flows," Chem. Eng. Research \& Design (1991) 69, No. 3, 190.

102. Meister, B.J. and Biggs, R.D.:: "Prediction of the First Normal Stress Difference in Polymer Solution," AIChE J (1969) 15, No. 5, 643.

103. Fried, F. et al: "First Normal Stress Difference and Viscosity in Shear of Liquid Crystalline Solutions of Hydroxypropyl-cellulose: New Experimental Data and Theory," Polymer (1994) 5, No. 9, 596.

104. Takahashi, Y. et al: "Shear-rate Dependence of First Normal Stress Difference of Poly (isoprene-b-styrene) in Solution near the Order-disorder Transition Temperature," Polymer (1996) 37 No. 26, 5943. 
105. Vlcek, J., and Bartos, O.: "Use of a Generalized Model Based on the Relaxation Spectrum for Calculation of the First Normal Stress Difference from a Flow Curve," J. of NonNewtonian Fluid Mechanics (1985) 19, No. 2, 113.

106. Wang Zhiwu: "Enhanced Oil Recovery Techniques and Their Application," Shanghai Transportation University, (April 1995) ISDN-7-313-01449-x/TE.01.

107. Wankui, G. et al.: "Commercial Pilot Test of Polymer Flooding in Daqing Oil Field," paper SPE 59275 presented at the 2000 SPE/DOE Symposium on Enhanced Oil Recovery, Tulsa, OK, April 3-6.

108. Wang D. et al.: "Producing by Polymer Flooding more than 300 Million Barrels of Oil, What Experiences Have Been Learnt?" paper SPE 77872 presented at the 2002 SPE Asia Pacific Oil and Gas Conference and Exhibition, Melbourne, Australia, Oct. 8-10.

109. Wang, D. et al.: "Summary of ASP Pilots in Daqing Oil Field," paper SPE 57288 presented at the 1999 SPE Asia Pacific Improved Oil Recovery Conference, Kuala Lumpur, Malaysia, Oct. 25-26.

110. Hou J. et al:: "Study of the Effect of ASP Solution Viscoelasticity on Displacement Efficiency," paper SPE 71492 presented at the 2001 SPE Annual Technical Conference and Exhibition, New Orleans, LA, Sep. 30-Oct. 3.

111. Wang, Dongmei: "Evaluation of Numerical Simulation Matching Study on the ASP Field Tests in Daqing," presented at the 2003 International Symposium on Petroleum High Efficiency Exploit of Oil and Gas, Bejing, China, Sep.29-Oct.1.

112. Sorbie, K.S. and Seright, R.S.: "Gel Placement in Heterogeneous Systems with Crossflow," paper SPE 24192 presented at the 1992 SPE/DOE Symposium on Enhanced Oil Recovery, Tulsa, OK, April 22-24.

113. Moradi-Araghi, A. and Doe, P.H.: "Hydrolysis and Precipitation of Polyacrylamides in Hard Brines at Elevated Temperatures," SPERE (May 1987) 189-197.

114. Ryles, R.G.: "Chemical Stability Limits of Water-Soluble Polymers Used in Oil Recovery Processes," SPERE (Feb. 1988) 23-34.

115. Seright, R.S.: "Aperture-Tolerant, Chemical-Based Methods to Reduce Channeling," Annual Technical Progress Report (U.S. DOE Report DOE/NT/15519-2), U.S. DOE Contract DE-FC26-04NT15519 (Sep. 2005).

116. Chang, H.L. et al.: "Successful Field Pilot of In-Depth Colloidal Dispersion Gel (CDG) Technology in Daqing Oil Field," SPE paper 89460 presented at the 2004 SPE/DOE Symposium on Improved Oil Recovery, Tulsa, OK, April 17-20.

117. Smith, J.E., Liu, H., and Guo, Z.D.: "Laboratory Studies of In-Depth Colloidal Dispersion Gel Technology for Daqing Oil Field," SPE paper 62610 presented at the 2000 SPE/AAPG Western Regional Meeting, Long Beach, CA, June 19-23.

118. Mack, J.C. and Smith, J.E.: "In-Depth Colloidal Dispersion Gels Improve Oil Recovery Efficiency," paper SPE 27780 presented at the 1994 SPE/DOE Symposium on Improved Oil Recovery, Tulsa, OK, April 17-20.

119. Fielding, R.C., Gibbons, D.H., and Legrand, F.P.: "In-Depth Drive Fluid Diversion Using and Evolution of Colloidal Dispersion Gels and New Bulk Gels: An Operational Case History of North Rainbow Ranch Unit," paper SPE 27773 presented at the 1994 SPE/DOE Symposium on Improved Oil Recovery, Tulsa, OK, April 17-20.

120. Hejri, S. et al.: "Permeability Reduction by a Xanthan/Cr(III) System in Porous Media," SPERE (Nov. 1993) 299-304. 
121. Todd, B.J., Green, D.W., and Willhite, G.P.: "A Mathematical Model of In-Situ Gelation of Polyacrylamide by a Redox Process," SPERE (Feb. 1993) 51-58.

122. Seright, R.S.: "Gel Placement in Fractured Systems," SPEPF (Nov. 1995), 241-248.

123. Seright, R.S. and Martin, F.D.: "Effect of $\mathrm{Cr}^{3+}$ on the Rheology of Xanthan Formulations in Porous Media: Before and After Gelation," In Situ (1992) 16 (1), 1-16.

124. Walsh, M.P. et al.: "Chemical Interactions of Aluminum-Citrate Solutions with Formation Minerals," paper SPE 11799 presented at the 1983 SPE International Symposium on Oilfield Chemistry, Denver, CO, June 1-3.

125. Rocha, C.A. et al.: "An Experimental Study of the Interactions of Aluminum Citrate Solutions and Silica Sand," paper SPE 18503 presented at the 1989 SPE International Symposium on Oilfield Chemistry, Houston, TX, Feb. 8-10.

126. Ranganathan, R. et al:: "Experimental Study of the Gelation Behavior of a Polyacrylamide/Aluminum Citrate Colloidal-Dispersion Gel System," SPEJ (Dec. 1998) 337-343.

127. Fletcher, A.J.P. et al.: "Deep Diverting Gels for Very Cost-Effective Waterflood Control," J. Petr. Sci. Eng. 7 (1992) 33-43.

128. Stavland, A. and Johsbraten, H.C.: "New Insight into Aluminum Citrate/Polyacrylamide Gels for Fluid Control," paper SPE/DOE 35381 presented at the 1996 SPE/DOE Symposium on Improved Oil Recovery, Tulsa, OK, April 21-24.

129. Seright, R.S.: "Impact of Dispersion on Gel Placement for Profile Control," SPERE (Aug. 1991) 343-352.

130. Bai, B. et al.: "Preformed Particle Gel for Conformance Control: Factors Affecting its Properties and Applications," paper SPE 89389 presented at the 2004 SPE/DOE Symposium on Improved Oil Recovery, Tulsa, OK, April 17-21.

131. Bai, B. et al.: "Preformed Particle Gel for Conformance Control: Transport Mechanism Through Porous Media," paper SPE 89468 presented at the 2004 SPE/DOE Symposium on Improved Oil Recovery, Tulsa, OK, April 17-21.

132. Seright, R.S.: "The Effects of Mechanical Degradation and Viscoelastic Behavior on Injectivity of Polyacrylamide Solutions," SPEJ (June 1983) 475-485.

133. Trantham, J.C., Threlkeld, C.B., and Patternson, H.L.: "Reservoir Description for a Surfactant/Polymer Pilot in a Fractured, Oil-Wet Reservoir-North Burbank Unit Tract 97," JPT (Sep. 1980) 1647-1656.

134. Crawford, F.B.and Collins, R.E.: "Estimated Effect of Vertical Fractures on Secondary Recovery," Trans., AIME (1954) 201, 192.

135. Dyes, A.B., Kemp, C.E., and Caudle, B.H.: "Effect of Fractures on Sweep-Out Pattern," Trans., AIME (1958) 213, 245.

136. Knight, B.L.: "Reservoir Stability of Polymer Solutions" JPT (May 1973) 618-626.

137. Shupe, R.D.: "Chemical Stability of Polyacrylamide Polymers," JPT (Aug. 1981) 1513-1529.

138. Morris, C.W. and Jackson, K.M.: "Mechanical Degradation of Polyacrylamide Solutions in Porous Media," paper SPE 7064 presented at the 1978 Fifth Symposium on Improved Methods for Oil Recovery, Tulsa, OK, April 16-19.

139. Wang, D. et al: "Study of the Mechanism of Polymer Solution with Visco-Elastic Behavior Increasing Microscopic Oil Displacement Efficiency and the Forming of Steady Oil Thread Flow Channels," paper SPE 68723 presented at the 2001 SPE Asia Pacific Oil and Gas Conference and Exhibition, Jakarta, Indonesia, April 17-19. 
140. Yang, Fulin et al:: "High Concentration Polymer Flooding is Successful," paper SPE 88454 presented at the 2004 SPE Asia Pacific Oil and Gas Conference and Exhibition, Perth, Australia, Oct. 18-20.

141. Luo Jianhui, etc. "Performance Properties of Salt Tolerant Polymer KYPAM for EOR", Oilfield Chemistry, 19(1) (March, 2002) 25.

142. Luo Jianhui and Zhu Huaijiang: "Comb Polymer Used in Polymer Flooding," paper presented at the 2005 SPE Applied Technology Workshop on Chemical Flooding, Daqing, China, Sep. 24-29.

143. Luo Pingya and Guo Yongjun: "Properties and Application of Water-Soluble Hydrophobic Associative Polymer AP-P4 for Polymer Flooding," paper presented at the 2005 SPE Applied Technology Workshop on Chemical Flooding, Daqing, China, Sep. 24-29.

144. Maitin, B.K.: "Performance Analysis of Several Polyacrylamide Floods in North German Oil Fields," paper SPE 24118 presented at the 1992 SPE/DOE Symposium on Improved Oil Recovery, Tulsa, OK, April 22-24.

145. Alvarez, J.M., Rivas, H., and Rossen, W.R.: "A Unified Model for Steady-State Foam Behavior at High and Low Foam Qualities," SPEJ 6 (Sep. 2001), 325-333.

146. Nimir, H.B. and Seright, R.S.: "Placement Properties of Foams Versus Gelants When Used as Blocking Agents," paper SPE 35172 presented at the 1996 SPE Permian Basin Oil \& Gas Recovery Conference, Midland, TX, Mar. 27-29.

147. Khatib, Z.I., Hirasaki, G.J., and Falls, A.H.: "Effects of Capillary Pressure on Coalescence and Phase Mobilities in Foams Flowing Through Porous Media," SPERE (Aug. 1988) 919926.

148. Wang D. et al:: "Successful Field Test of the First Ultra-Low Interfacial Tension Foam Flood," paper SPE 72147 presented at the 2001 SPE Asia Pacific Improved Oil Recovery Conference, Kuala Lumpur, Malaysia, Oct. 6-9.

149. Schramm, L.L.: Foams: Fundamentals and Applications in the Petroleum Industry, American Chemical Society, Washington, DC (1994).

150. Hanssen, J.E. and Dalland, M.: "Increased Oil Tolerance of Polymer-Enhanced Foams: Deep Chemistry or Just Simple Displacement Effects?," paper SPE 59282 presented at the 2000 SPE/DOE Improved Oil Recovery Symposium, Tulsa, OK, April 3-5.

151. Liu, Y., Grigg, R.B., and Bai, B.: "Salinity, pH, and Surfactant Concentration Effects on $\mathrm{CO}_{2}$-Foam," paper SPE 93095 presented at the 2005 SPE International Symposium on Oilfield Chemistry, Houston, TX, Feb. 2-4.

152. Lake, L.W.: Enhanced Oil Recovery, Prentice Hall, Englewood Cliffs, NJ (1989) 424-449.

153. Wang, J., Cheng, D., and Wang, D.: "Combining Small Well Spacing with Polymer Flooding to Improve Oil Recovery of Marginal Reservoirs," paper SPE 96946 presented at the 2006 SPE Improved Oil Recovery Symposium, Tulsa, OK, April 22-26.

154. Chang, H.L., et al.: "Advances in Polymer Flooding and Alkaline/Surfactant/Polymer Processes as Developed and Applied in the People's Republic of China," JPT (February 2006) 84-89.

155. Al-Assi, A.A. et al:: "Formation and Propagation of Gel Aggregates Using Partially Hydrolyzed Polyacrylamide and Aluminum Citrate," paper SPE 100049 presented at the 2006 SPE/DOE Symposium on Improved Oil Recovery, Tulsa, OK, April 22-26.

156. Seright, R.S.: "Improved Techniques for Fluid Diversion in Oil Recovery Processes," second annual report (DOE/BC/14880-10), Contract No. DE-AC22-92BC14880, U.S. DOE (March 1995), 51-64. 
157. Wang, D., et al..: "Sweep Improvement Options for the Daqing Oil Field," paper SPE 99441 presented at the 2006 SPE/DOE Symposium on Improved Oil Recovery, Tulsa, OK, April 22-26.

158. Seright, R.S.: "Conformance Improvement Using Gels," Annual Technical Progress Report (U.S. DOE Report DOE/BC/15316-6), U.S. DOE Contract DE-FC26-01BC15316 (Sept. 2004) 72.

159. Norman, C., De Lucia, J., and Turner, B.: "Improving Volumetric Sweep Efficiency With Polymer Gels in the Cuyo Basin of Argentina," paper SPE 99379 presented at the 2006 SPE/DOE Symposium on Improved Oil Recovery, Tulsa, OK, April 22-26.

160. Seright, R.S.: "Washout of Cr(III)-Acetate-HPAM Gels from Fractures," paper SPE 80200 presented at the 2003 SPE International Symposium on Oilfield Chemistry, Houston, TX, Feb. 5-7.

161. Smith, D.H.: Surfactant-Based Mobility Control, ACS Symposium Series 373, American Chemical Society, Washington, DC (1988).

162. Schramm, L.L.: Foams: Fundamentals and Applications in the Petroleum Industry, Advances in Chemistry Series 242, American Chemical Society, Washington, DC (1994).

163. Kim, J.S., Dong, Y., and Rossen, W.R.: "Steady-State Foam Behavior of $\mathrm{CO}_{2}$ Foams," paper SPE 89351 presented at the 2004 SPE/DOE Symposium on Improved Oil Recovery, Tulsa, OK, April 17-21.

164. Seright, R.S.: "Effect of Rheology on Gel Placement," SPE Reservoir Engineering (May 1991), 212-218; Transactions AIME 291.

165. Koval, E.J.: "A Method for Predicting the Performance of Unstable Miscible Displacement in Heterogeneous Porous Media," SPEJ (June 1963) 143-155. Transactions AIME, 228.

166. Pittaway, K.R.: "Physical Properties of Carbon Dioxide", Research Report 102-8-4-1-79 (Nov. 1979).

167. Shan, D., and Rossen, W.R.: “Optimal Injection Strategies for Foam IOR," SPEJ 9(2) (June 2004) 132-150.

168. Grigg, R.B. and Bai, B.: "Sorption of Surfactant Used in CO2 Flooding onto Five Minerals and Three Porous Media," paper SPE 93100 presented at the 2005 SPE International Symposium on Oilfield Chemistry, The Woodlands, TX, Feb. 2-4.

169. Pye, D.J.: "Improved Secondary Recovery by Control of Water Mobility," JPT (Aug. 1964) 911-916.

170. Smith, F.W.: "The Behavior of Partially Hydrolyzed Polyacrylamide Solutions in Porous Media," JPT (Feb. 1970) 148-156.

171. Jewett, R.L. and Schurz G.F.: "Polymer Flooding-A Current Appraisal," JPT (June 1979) 675-684.

172. Seright, R.S., Maerker, J.M., and Holzwarth G.: "Mechanical Degradation of Polyacrylamides Induced by Flow Through Porous Media," American Chemical Society Polymer Preprints, Vol. 22 (Aug. 1981) 30-33.

173. Coats, K.H. et al.: "Simulation of Three-Dimensional, Two-Phase Flow in Oil and Gas Reservoir," SPEJ (Dec. 1967) 377-388.

174. Coats, K.H., Dempsey, J.R., and Henderson, J.H.: "The Use of Vertical Equilibrium in Two-Dimensional Simulation of Three-Dimensional Reservoir Performance," SPEJ (March 1971) 63-71. 
175. Zapata, V.J. and Lake, L.W.: "A Theoretical Analysis of Viscous Crossflow." Paper SPE 10111 presented at the 1981 SPE Annual Technical Conference and Exhibition, San Antonio, TX, Oct. 5-7. 


\section{APPENDIX A: Technology Transfer}

\section{Presentations}

On September 11-15, 2006, we presented the course, "Strategies for Water Problem Diagnosis and Best Practices of Water Shutoff" in Mexico City, Mexico.

On June 27, 2006, we presented the poster, "Optimizing Disproportionate Permeability Reduction," at the 2006 SPE Form on "Enhanced Oil Recovery, What's Next?" at Bloomfield, $\mathrm{CO}$.

On June 22, 2006, we presented the workshop, "Polymer and Polymer-Gel Water Shutoff Treatments: What It Takes to Be Successful and Illustrative Field Applications", at Midland College, Midland, TX.

On June 21, 2006, we presented "Useful Website for Water Shutoff" for the Monthly SIPES meeting, Midland, TX.

On May 29, 2006, we presented "X-Ray Computed Microtomography Studies of Disproportionate Permeability Reduction," at SINTEF, Trondheim, Norway.

On May 29, 2006, we presented "Problems with Disproportionate Permeability Reduction by Adsorbed Polymers and Weak Gels," at Statoil, Trondheim, Norway.

On April 25, 2006, we presented SPE 99441, "Sweep Improvement Options for the Daqing Oil Field," at the 2006 SPE/DOE Symposium on Improved Oil Recovery, Tulsa, OK.

On April 25, 2006, we presented SPE 99462, "Correlating Gel Rheology with Behavior during Extrusion through Fractures," at the 2006 SPE/DOE Symposium on Improved Oil Recovery, Tulsa, OK.

On April 25, 2006, we presented SPE 99371, "When and Where Relative Permeability Modification Water-Shutoff Treatments Can Be Successfully Applied," at the 2006 SPE/DOE Symposium on Improved Oil Recovery, Tulsa, OK.

On April 24, 2006, we presented SPE 99443, "Optimizing Disproportionate Permeability Reduction," at the 2006 SPE/DOE Symposium on Improved Oil Recovery, Tulsa, OK.

On April 23, 2006, we presented the course, "Water Shutoff” in Tulsa, OK.

On December 6, 2005, we presented the talk, "Optimizing Disproportionate Permeability Reduction," at the University of Kansas, Lawrence, KS.

On September 27, 2005, we presented the talk, "Physical Realities for In-Depth Profile Modification," at the SPE Applied Technology Workshop on Chemical Flooding in Daqing, China. 
On February 9-10, 2005, we presented the Workshop, "Polymer and Polymer-Gel Water Shutoff Treatments: What It Takes to Be Successful and Illustrative Field Applications," at the University of WY Enhanced Oil Recovery Institute in Laramie, WY.

On February 2, 2005, we presented the talk, "Clean Up of Oil Zones after a Gel Treatment," at the 2005 SPE International Symposium on Oilfield Chemistry in Houston, TX.

On November 8-12, 2004, we presented the course, "Water Shutoff” in Mexico City, Mexico.

On October 20, 2004, we presented the talk, "Throughput Dependence of Oil and Water Permeabilities after Treatment with Gel or Polymer," at the $3^{\text {rd }}$ International Conference on Oil and Gas Development in Chengdu, China.

On October 19, 2004, we presented the talk, "Challenges for the Development of Improved Mobility Control Methods," for the China Institute for Organic Chemistry in Chengdu, China.

On October 13-14, 2004, we presented the talk, "Three Approaches for Improving Oil Recovery," for the Research Institute of Exploration and Development of Daqing Oil Field, PetroChina in Daqing, China.

On August 25, 2004, we presented the PTTC Workshop, "Polymer and Polymer-Gel Water Shutoff Treatments: What It Takes to Be Successful and Illustrative Field Applications", at the TX Bureau of Economic Geology facility in Houston, TX.

\section{Web Site}

A description of our research group can be found at the following New Mexico PRRC/Tech web site: http://baervan.nmt.edu/randy. For those new to water shutoff technology, this site provides an extensive introduction. This introduction details the properties of polymers, gelants, and gels - showing what they can and cannot do. Many myths associated with this technology are dispelled. The introduction also provides guidance on where and how gel treatments should (and should not) be applied. For many different types of water shutoff problems, we discuss relevant field examples, including (1) how to diagnose the problem, (2) what gel properties are needed, (3) how much gel should be injected, (4) how the gel should be placed, and (5) how to assess the effectiveness of the treatment.

The web site also describes a strategy for attacking excess water production problems. Many oil and gas producers realize that identifying the problem is critical before attempting a solution, but most producers have limited resources for diagnosis. The site details which problems should be looked for first, which problems are easy to solve, and which are more difficult.

Important equations for use in designing gel treatments are also included. Spreadsheets that perform these important calculations can be readily downloaded from our web site.

For those with more expertise in water shutoff, the site provides a detailed review of the intricacies of gel placement. It also provides discussion of special topics of current interest. For 
example, "Clean Up of Oil Zones after a Gel Treatment" is of high interest for those who bullhead gel treatments into production wells. Also, "A New Filter Cake Model" is of interest to those involved with hydraulic fracturing and produced water re-injection, as well as to those who inject large gel volumes during water shutoff applications. Videos are provided that illustrate basic principles of polymer flooding and crossflow.

Access is provided to annual reports for our work in water shutoff back to 1990. A search engine is included that covers all our reports and publications. This web site is a valuable resource for anyone who needs to reduce excess salt water production during their oil and gas operations.

\section{Papers and Publications}

Prodanovic, M., Lindquist, W.B., and Seright, R.S.: "Porous Structure and Fluid Partitioning in Polyethylene Cores from 3D X-ray Microtomographic Imaging," J. Colloid and Interface Science 298 (June 2006) 282-297.

Prodanovic, M., Lindquist, W.B., and Seright, R.S.: "3D Image-Based Characterization of Fluid Displacement in a Berea Core," Advances in Water Resources (in press) 2006.

Seright, R.S., Prodanovic, M., and Lindquist, W.B.: "X-Ray Computed Microtomography Studies of Fluid Partitioning in Drainage and Imbibition Before and After Gel Placement: Disproportionate Permeability Reduction," SPE Journal (June 2006) 159-170.

Prodanovic, M., Lindquist, W.B., and Seright, R.S.: "Residual Fluid Blobs and Contact Angle Measurements from X-Ray Images of Fluid Displacement," presented at the Computational Methods in Water Resources XVI Conference, Copenhagen, Denmark, June 19-22, 2006.

Seright, R.S.: "Clean Up of Oil Zones after a Gel Treatment," SPE Production \& Operations (May 2006) 237-244.

Seright, R.S.: “Are Colloidal Dispersion Gels Really a Viable Technology?", Journal of Petroleum Technology (May 2006) 80.

Seright, R.S.: "Optimizing Disproportionate Permeability Reduction," paper SPE 99443 presented at the 2006 SPE/DOE Symposium on Improved Oil Recovery, Tulsa, OK, April 22-26.

Wang, Dongmei; Han, Peihui; Shao, Zhenbo; Chen, Jinfeng; and Seright, R.S.: "Sweep Improvement Options for the Daqing Oil Field," paper SPE 99441 presented at the 2006 SPE/DOE Symposium on Improved Oil Recovery, Tulsa, OK, April 22-26.

Wang, Ying, and Seright, R.S.: "Correlating Gel Rheology with Behavior during Extrusion through Fractures," paper SPE 99462 presented at the 2006 SPE/DOE Symposium on Improved Oil Recovery, Tulsa, OK, April 22-26. 
Sydansk, R.D., and Seright, R.S.: "When and Where Relative Permeability Modification WaterShutoff Treatments Can Be Successfully Applied," paper SPE 99371 presented at the 2006 SPE/DOE Symposium on Improved Oil Recovery, Tulsa, OK, April 22-26.

Prodanovic, M., Lindquist, W.B., and Seright, R.S.: "3D Image-Based Characterization of Fluid Displacement in a Berea Core," 2005, Accepted by Advances in Water Resources.

Sydansk, R.D., Al-Dhafeeri, A., Xiong, Y., Schrader, R., and Seright, R.S.: "Characterization of Partially Formed Polymer Gels for Application to Fractured Production Wells for Water-Shutoff Purposes," SPE Production \& Facilities (Aug. 2005), 20(3) 240-249.

Seright, R.S.: "Clean Up of Oil Zones after a Gel Treatment," paper SPE 92772 presented at the 2005 SPE International Symposium on Oilfield Chemistry, Houston, TX, Feb. 2-4.

Sydansk, R.D., Al-Dhafeeri, A.M., Xiong, Y., and Seright, R.S.: "Polymer Gels Formulated with a Combination of High- and Low-Molecular-Weight Polymers Provide Improved Performance for Water-Shutoff Treatments of Fractured Production Wells," SPE Production \& Facilities (Nov. 2004), 19(4) 229-236.

Seright, R.S.: "Throughput Dependence of Oil and Water Permeabilities after Treatment with Gel or Polymer," $3^{\text {rd }}$ International Conference on Oil and Gas Development, Chengdu, China, Oct. 20-21, 2004. 


\section{APPENDIX B: Derivation of Penetration Equations for Floods with Newtonian Fluids}

\section{Assumptions:}

1. Fluids are incompressible and Newtonian.

2. Displacement is miscible and piston-like.

3. Dispersion and adsorption are negligible.

4. All layers have the same dimensions in the horizontal direction and experience the same total pressure drop.

5. Only aqueous fluids are mobile in the region to be contacted by gelling agent.

6. $\mathrm{F}_{\mathrm{r}}$ (resistance factor) is independent of permeability.

7. The position of the oil-water front (if present) does not move significantly during the gel placement process.

8. Gelling agent will propagate the greatest distance in layer 1.

9. No crossflow occurs between layers.

\section{Linear Floods:}

$\left.\Delta p=\left[q_{i} / A_{i} k_{i}\right)\right]\left[\mu_{p} L_{p i}+\mu_{w}\left(L_{p m}-L_{p i}\right)+\Psi_{i} \mu_{w} L_{p m}\right]$

and

$F_{r}=\left(k_{i} / \mu_{w}\right) /\left(k_{i} / \mu_{p}\right)$

so

$\Delta p=\left[q_{i} \mu_{w} /\left(A_{i} k_{i}\right)\right]\left[F_{r} L_{p i}+\left(L_{p m}-L_{p i}\right)+\Psi_{i} L_{p m}\right]$

Also,

$q_{i}=A_{i} \phi_{i}\left(d L_{p i} / d t\right)$

where $\Delta p=$ pressure drop across the layers,

$q_{i}=$ volumetric flow rate in layer $\mathbf{i}$,

$A_{i}=$ cross-sectional area of layer $\mathbf{i}$,

$k_{i}=$ permeability of layer $\mathbf{i}$,

$\phi_{\mathrm{i}}=$ porosity of layer $\mathbf{i}$,

$L_{p i}=$ distance of penetration into layer $\mathbf{i}$,

$L_{p m}=$ maximum distance of penetration of gelling agent into the most permeable layer (layer 1),

$\mu_{\mathrm{p}}=$ viscosity of the gelling formulation,

$\mu_{\mathrm{w}}=$ water viscosity,

$\Psi_{\mathrm{i}}=$ pressure drop between $\mathrm{L}_{\mathrm{pm}}$ and the end of layer $\mathbf{i}$ divided by the pressure drop between the beginning of layer $\mathbf{i}$ and $\mathrm{L}_{\mathrm{pm}}$ prior to injecting gelling agents, and

So,

$t=$ time. 
$\Delta p=\mu_{w}\left(\phi_{i} / k_{i}\right)\left[\left(F_{r}-1\right) L_{p i}+\left(\Psi_{i}+1\right) L_{p m}\right]\left(d L_{p i} / d t\right)$

$\Delta p$ is the same across layer $\mathbf{1}$ and layer $\mathbf{i}$, so

$\left(\phi_{i} / k_{i}\right)\left[\left(F_{r}-1\right) L_{p i}+\left(\Psi_{i}+1\right) L_{p m}\right]\left(d L_{p i} / d t\right)=\left(\phi_{1} / k_{1}\right)\left[\left(F_{r}-1\right) L_{p 1}+\left(\Psi_{1}+1\right) L_{p m}\right]\left(d L_{p 1} / d t\right)$

Multiplying by $d t$

$\left(\phi_{i} / k_{i}\right)\left[\left(F_{r}-1\right) L_{p i}+\left(\Psi_{i}+1\right) L_{p m}\right] d L_{p i}=\left(\phi_{1} / k_{1}\right)\left[\left(F_{r}-1\right) L_{p 1}+\left(\Psi_{1}+1\right) L_{p m}\right] d L_{p 1}$

Integrating the left side of the equation between 0 and $L_{p i}$ while integrating the right side between 0 and $L_{p 1}$ yields,

$\left(\phi_{i} / k_{i}\right)\left[\left(F_{r}-1\right) L_{p i}^{2} / 2+\left(\Psi_{i}+1\right) L_{p m} L_{p i}\right]=\left(\phi_{1} / k_{1}\right)\left[\left(F_{r}-1\right) L_{p l}^{2} / 2+\left(\Psi_{1}+1\right) L_{p m} L_{p 1}\right]$

Let $L_{p l}=L_{p m}$,

$\left(\phi_{i} / k_{i}\right)\left[\left(F_{r}-1\right) L_{p i}{ }^{2} / 2+\left(\Psi_{i}+1\right) L_{p m} L_{p i}\right]=\left(\phi_{1} / k_{1}\right)\left[\left(F_{r}-1\right) L_{p m}{ }^{2} / 2+\left(\Psi_{1}+1\right) L_{p m}{ }^{2}\right]$

Solving for $L_{p i} / L_{p m}$,

$L_{p i} / L_{p m},=\left\{\left[\left(\Psi_{i}+1\right)^{2}+\left(\phi_{1} / k_{1}\right)\left(k_{i} / \phi_{i}\right)\left(F_{r}-1\right)\left(F_{r}+2 \Psi_{1}+1\right)\right]^{1 / 2}-\left(\Psi_{i}+1\right)\right\} /\left(F_{r}-1\right)$

For linear corefloods $\Psi_{1}=\Psi_{i}=0$ and $L_{p m}=L_{t}$ (total core length), so

$L_{p i} / L_{t}=\left\{\left[1+\left(F_{r}^{2}-1\right)\left(\phi_{1} / k_{1}\right)\left(k_{i} / \phi_{i}\right)\right]^{1 / 2}-1\right\} /\left(F_{r}-1\right)$

\section{$\underline{\text { Radial Floods: }}$}

$\Delta p=q_{i}\left[\mu_{p} \ln \left(r_{p i} / r_{o}\right)+\mu_{w} \ln \left(r_{p m} / r_{p i}\right)+\Psi_{i} \mu_{w} \ln \left(r_{p m} / r_{o}\right)\right] /\left(2 \pi k_{i} h_{i}\right)$

Using the definition of resistance factor $\left(F_{r}\right)$

$\Delta p=q_{i} \mu_{w}\left[F_{r} \ln \left(r_{p i} / r_{o}\right)+\ln \left(r_{p m} / r_{p i}\right)+\Psi_{i} \ln \left(r_{p m} / r_{o}\right)\right] /\left(2 \pi k_{i} h_{i}\right)$

Also,

$q_{i}=2 \pi h_{i} \phi_{i} r_{p i}\left(d r_{p i} / d_{t}\right)$

where $r_{p i}=$ radius of penetration of gelling agent in layer $\mathbf{i}$,

$r_{o}=$ wellbore radius,

$r_{p m}=$ maximum radius of penetration of gelling agent in the most permeable layer (layer 1),

$h_{i}=$ height of layer $\mathbf{1}$, and 
$\Psi_{i}=$ pressure drop between $r_{p m}$ and production well divided by pressure drop between injection well and $r_{p m}$ (in layer i), so,

$$
\Delta p=\left(\phi_{i} / k_{i}\right) \mu_{w}\left[F_{r} \ln \left(r_{p i} / r_{o}\right)+\ln \left(r_{p m} / r_{p i}\right)+\Psi_{i} \ln \left(r_{p m} / r_{o}\right)\right] r_{p i}\left(d r_{p i} / d_{t}\right)
$$

$\Delta p$ is the same across layer $\mathbf{1}$ and layer $\mathbf{i}$, so

$$
\begin{aligned}
\left(\phi_{i} / k_{i}\right)\left[F_{r} \ln \left(r_{p i} / r_{o}\right)+\ln \left(r_{p m} / r_{p i}\right)+\Psi_{i} \ln \left(r_{p m} / r_{o}\right)\right] r_{p i}\left(d r_{p i} / d_{t}\right)= \\
\left(\phi_{1} / k_{1}\right)\left[F_{r} \ln \left(r_{p 1} / r_{o}\right)+\ln \left(r_{p m} / r_{p 1}\right)+\Psi_{1} \ln \left(r_{p m} / r_{o}\right)\right] r_{p 1}\left(d r_{p 1} / d_{t}\right)
\end{aligned}
$$

Multiplying by $d t$

$$
\begin{aligned}
& \left(\phi_{i} / k_{i}\right)\left[F_{r} \ln \left(r_{p i} / r_{o}\right)+\ln \left(r_{p m} / r_{p i}\right)+\Psi_{i} \ln \left(r_{p m} / r_{o}\right)\right] r_{p i}\left(d r_{p i}\right)= \\
& \left(\phi_{1} / k_{1}\right)\left[F_{r} \ln \left(r_{p 1} / r_{o}\right)+\ln \left(r_{p m} / r_{p 1}\right)+\Psi_{1} \ln \left(r_{p m} / r_{o}\right)\right] r_{p 1}\left(d r_{p 1}\right)
\end{aligned}
$$

Expanding the log terms

$$
\begin{aligned}
&\left(\phi_{i} / k_{i}\right) {\left[\left(F_{r}-1\right) r_{p i} \ln \left(r_{p i}\right)+r_{p i}\left(\ln \left(r_{p m}\right)-F_{r} \ln \left(r_{o}\right)+\Psi_{i} \ln \left(r_{p m} / r_{o}\right)\right)\right] d r_{p i}=} \\
&\left(\phi_{1} / k_{1}\right)\left[\left(F_{r}-1\right) r_{p 1} \ln \left(r_{p 1}\right)+r_{p 1}\left(\ln \left(r_{p m}\right)-F_{r} \ln \left(r_{o}\right)+\Psi_{1} \ln \left(r_{p m} / r_{o}\right)\right)\right] d r_{p 1}
\end{aligned}
$$

Integrating the left side of this equation between $r_{o}$ and $r_{p l}$ while integrating the right side between $r_{o}$ and $r_{p 1}$ gives

$$
\begin{aligned}
\left(\phi_{i} / k_{i}\right) & \left\{\left(F_{r}-1\right)\left[\left(r_{p i}^{2} / 2\right) \ln \left(r_{p i}\right)-r_{p i}^{2} / 4\right]+\left[\ln \left(r_{p m}\right)-F_{r} \ln \left(r_{o}\right)\right]+\Psi_{i} \ln \left(r_{p m} / r_{o}\right)\right] r_{p i}{ }^{2} / 2 \\
& \left.-\left(F_{r}-1\right)\left[\left(r_{o}^{2} / 2\right) \ln \left(r_{o}\right)-r_{o}{ }^{2} / 4\right]-\left[\ln \left(r_{p m}\right)-F_{r} \ln \left(r_{o}\right)+\Psi_{i} \ln \left(r_{p m} / r_{o}\right)\right] r_{o}{ }^{2} / 2\right\}= \\
& \left(\phi_{1} / k_{1}\right)\left\{\left(F_{r}-1\right)\left[\left(r_{p 1}{ }^{2} / 2\right) \ln \left(r_{p 1}\right)-r_{p 1}{ }^{2} / 4\right]+\left[\ln \left(r_{p m}\right)-F_{r} \ln \left(r_{o}\right)+\Psi_{1} \ln \left(r_{p m} / r_{o}\right)\right] r_{p 1}{ }^{2} / 2\right. \\
& \left.-\left(F_{r}-1\right)\left[\left(r_{o}^{2} / 2\right) \ln \left(r_{o}\right)-r_{o}{ }^{2} / 4\right]-\left[\ln \left(r_{p m}\right)-F_{r} \ln \left(r_{o}\right)+\Psi_{1} \ln \left(r_{p m} / r_{o}\right)\right] r_{o}{ }^{2} / 2\right\}
\end{aligned}
$$

Multiplying by 2 and rearranging,

$$
\begin{aligned}
\left(\phi_{i} / k_{i}\right) & \left\{r_{p i}^{2}\left[F_{r} \ln \left(r_{p i} / r_{o}\right)+\ln \left(r_{p m} / r_{p i}\right)+\left(1-F_{r}\right) / 2+\Psi_{i} \ln \left(r_{p m} / r_{o}\right)\right]\right. \\
& \left.-r_{o}^{2}\left[\ln \left(r_{p m} / r_{o}\right)+\left(1-F_{r}\right) / 2+\Psi_{i} \ln \left(r_{p m} / r_{o}\right)\right]\right\}= \\
& \left(\phi_{1} / k_{1}\right)\left\{r_{p 1}{ }_{2}\left[F_{r} \ln \left(r_{p 1} / r_{o}\right)+\ln \left(r_{p m} / r_{p 1}\right)+\left(1-F_{r}\right) / 2+\Psi_{1} \ln \left(r_{p m} / r_{o}\right)\right]\right. \\
& \left.-r_{o}{ }^{2}\left[\ln \left(r_{p m} / r_{o}\right)+\left(1-F_{r}\right) / 2+\Psi_{1} \ln \left(r_{p m} / r_{o}\right)\right]\right\}
\end{aligned}
$$

Let $r_{p l}=r_{p m}$

$$
\begin{aligned}
&\left(\phi_{i} / k_{i}\right)\left\{r_{p i}^{2}\left[F_{r} \ln \left(r_{p i} / r_{o}\right)+\ln \left(r_{p m} / r_{p i}\right)+\left(1-F_{r}\right) / 2+\Psi_{i} \ln \left(r_{p m} / r_{o}\right)\right]\right. \\
&\left.-r_{o}^{2}\left[\ln \left(r_{p m} / r_{o}\right)+\left(1-F_{r}\right) / 2+\Psi_{i} \ln \left(r_{p m} / r_{o}\right)\right]\right\}= \\
&\left(\phi_{1} / k_{1}\right)\left\{r_{p m}{ }^{2}\left[F_{r} \ln \left(r_{p m} / r_{o}\right)+\left(1-F_{r}\right) / 2+\Psi_{1} \ln \left(r_{p m} / r_{o}\right)\right]\right. \\
&\left.-r_{o}^{2}\left[\ln \left(r_{p m} / r_{o}\right)+\left(1-F_{r}\right) / 2+\Psi_{1} \ln \left(r_{p m} / r_{o}\right)\right]\right\}
\end{aligned}
$$

For radial corefloods, $\Psi_{1}=\Psi \mathrm{i}=0$ and $r_{p m}=r_{e}$, so 


$$
\begin{gathered}
\left(\phi_{i} / k_{i}\right)\left\{r_{p i}^{2}\left[F_{r} \ln \left(r_{p i} / r_{o}\right)+\ln \left(r_{e} / r_{p i}\right)+\left(1-F_{r}\right) / 2\right]-r_{o}^{2}\left[\ln \left(r_{e} / r_{o}\right)+\left(1-F_{r}\right) / 2\right]\right\}= \\
\left(\phi_{1} / k_{1}\right)\left\{r_{e}^{2}\left[F_{r} \ln \left(r_{e} / r_{o}\right)+\left(1-F_{r}\right) / 2-r_{o}^{2}\left[\ln \left(r_{e} / r_{o}\right)+\left(1-F_{r}\right) / 2\right]\right\}\right.
\end{gathered}
$$

Rearranging yields,

$$
\begin{aligned}
& \left(\phi_{i} / k_{i}\right) r_{p i}^{2}\left[F_{r} \ln \left(r_{p i} / r_{o}\right)+\ln \left(r_{e} / r_{p i}\right)+\left(1-F_{r}\right) / 2\right]= \\
& \left(\phi_{1} / k_{1}\right) r_{e}^{2}\left[F_{r} \ln \left(r_{e} / r_{o}\right)+\left(1-F_{r}\right) / 2\right]-r_{o}^{2}\left(\phi_{1} / k_{1}-\phi_{i} / k_{i}\right)\left[\ln \left(r_{e} / r_{o}\right)+\left(1-F_{r}\right) / 2\right]
\end{aligned}
$$

\section{Combined Linear-Radial Flood:}

In the linear-flow portion,

$$
\Delta p=\mu_{w}\left(\phi_{i} / k_{i}\right)\left[\left(F_{r}-1\right) L_{p i}+\left(\Psi_{i}+1\right) L_{p m}\right]\left(d L_{p i} / d t\right)
$$

In the radial-flow portion,

$$
\Delta p=\left(\phi_{i} / k_{i}\right) \mu_{w}\left[F_{r} \ln \left(r_{p i} / r_{o}\right)+\ln \left(r_{p m} / r_{p i}\right)+\Psi_{i} \ln \left(r_{p m} / r_{o}\right)\right] r_{p i}\left(d r_{p i} / d t\right)
$$

If the greatest degree of penetration of gelling agent will occur in a linear layer (layer $\mathbf{1}$ ), then

$$
\begin{gathered}
\left(\phi_{i} / k_{i}\right)\left[F_{r} \ln \left(r_{p i} / r_{o}\right)+\ln \left(r_{p m} / r_{p i}\right)+\Psi_{i} \ln \left(r_{p m} / r_{o}\right)\right] r_{p i} d r_{p i}= \\
\left(\phi_{1} / k_{1}\right)\left[\left(F_{r}-1\right) L_{p 1}+\left(\Psi_{1}+1\right) L_{p m}\right] d L_{p 1}
\end{gathered}
$$

Integrating the left side between $r_{o}$ and $r_{p i}$ and the right side between 0 and $L_{p 1}$ gives

$$
\begin{aligned}
\left(\phi_{i} / k_{i}\right)\left\{\left(F_{r}-1\right)\left[\left(r_{p i}{ }^{2} / 2\right) \ln \left(r_{p i}\right)-r_{p i}{ }^{2} / 4\right]+\left[\ln \left(r_{p m}\right)-F_{r} \ln \left(r_{o}\right)+\Psi_{i} \ln \left(r_{p m} / r_{o}\right)\right] r_{p i}{ }^{2} / 2\right. \\
\left.\left.-\left(F_{r}-1\right)\left[\left(r_{o}{ }^{2} / 2\right) \ln \left(r_{o}\right)-r_{o}{ }^{2} / 4\right]+\left[\ln \left(r_{p m}\right)-F_{r} \ln \left(r_{o}\right)+\Psi_{i} \ln \left(r_{p m} / r_{o}\right)\right] r_{o}{ }^{2} / 2\right]\right\}= \\
\left(\phi_{1} / k_{1}\right)\left[\left(F_{r}-1\right) L_{p 1}{ }^{2} / 2+\left(\Psi_{i}+1\right) L_{p m} L_{p 1}\right]
\end{aligned}
$$

Multiplying by 2 , letting $L_{p l}=L_{p m}$ and rearranging gives

$$
\begin{aligned}
&\left(\phi_{i} / k_{i}\right) r_{p i}{ }^{2}\left[F_{r} \ln \left(r_{p i} / r_{o}\right)+\ln \left(r_{p m} / r_{p i}\right)+\Psi_{i} \ln \left(r_{p m} / r_{o}\right)+\left(1-F_{r}\right) / 2\right]- \\
&\left(\phi_{i} / k_{i}\right) r_{o}{ }^{2}\left[\left(\Psi_{i}+1\right) \ln \left(r_{p m} / r_{o}\right)+\left(1-F_{r}\right) / 2\right]=\left(\phi_{1} / k_{1}\right) L_{p m}{ }^{2}\left(F_{r}+1+2 \Psi_{l}\right)
\end{aligned}
$$




\section{APPENDIX C: Derivation of Penetration Equations for Unstable Displacement in Zone 2}

\section{Assumptions:}

1. Same basic assumptions as in Appendix B.

2. In regions that are fully saturated with a given fluid (e.g., polymer), $\mathrm{F}_{\mathrm{r}}$ (resistance factor) is independent of permeability.

3. In regions where a high mobility fluid is displacing a low mobility fluid, the average resistance factor, $F_{\mu}$, is given by the quarter-power mixing rule (Eq. 43 ).

$F_{\mu}=\left[0.78+0.22\left(\lambda_{g w} / \lambda_{o w}\right)^{0.25}\right]^{4}$

\section{Linear Flow:}

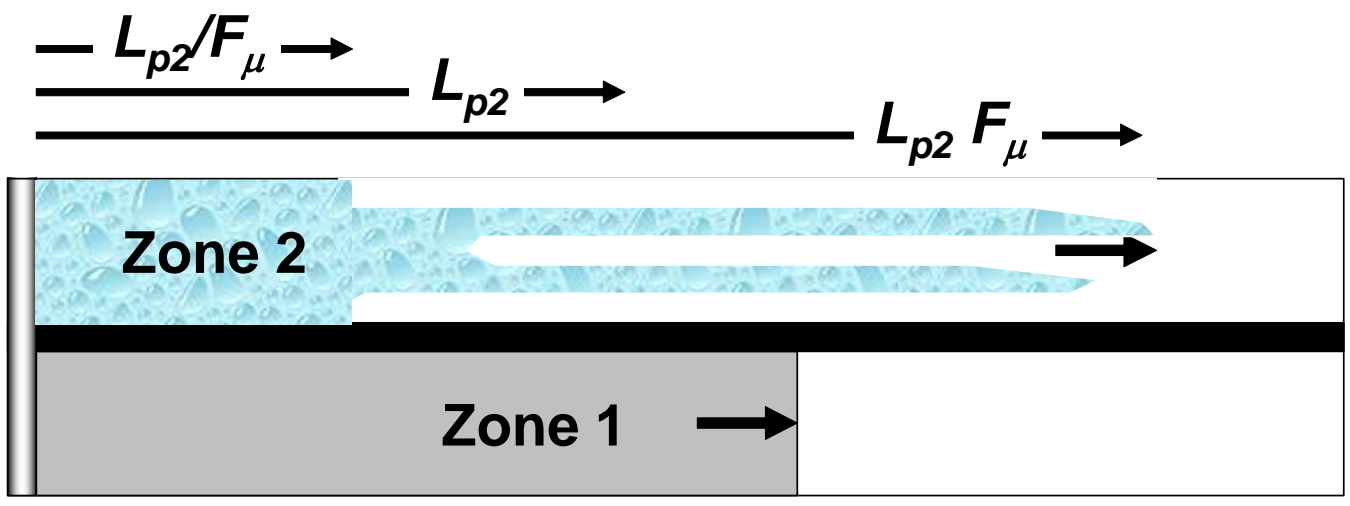

Fig. C-1-Gas/water flow in Zone 2 after foam forms in Zone 1. No Crossflow. Linear flow.

$q_{2}=A_{2} \phi_{2} d L_{p f} / d t=A_{2} \phi_{2} F_{\mu} d L_{w g} / d t=A_{2} \phi_{2} d L_{f f} / d t\left(1 / F_{\mu}\right)$

$\Delta p=\phi_{2} / k_{2} \quad\left[\mu_{w g} L_{w g}+\mu_{o w} F_{\mu}\left(L_{f f}-L_{w g}\right)+\mu_{o w}\left(L_{p m}-L_{f f}\right)+\mu_{o w} \Psi_{2} L_{p m}\right] d L_{p f} / d t$

From Refs. 129 and 165:

$$
\begin{aligned}
& L_{w g}=L_{p f} / F_{\mu} \\
& L_{f f}=L_{p f} F_{\mu} \\
& L_{w g}=L_{f f} /\left(F_{\mu}\right)^{2} \\
& L_{f f}=L_{w g} F_{\mu}{ }^{2} \\
& \Delta p=\left(\phi_{2} / k_{2}\right) F_{\mu}\left[\mu_{w g} L_{w g}+\mu_{o w} F_{\mu}\left(L_{w g} F_{\mu}{ }^{2}-L_{w g}\right)+\mu_{o w}\left(L_{p m}-F_{\mu}{ }^{2} L_{w g}\right)+\mu_{o w} \Psi_{2} L_{p m}\right]\left(d L_{w g} / d t\right) \\
& \Delta p=\left(\phi_{2} / k_{2}\right) F_{\mu}\left[L_{w g}\left(\mu_{w g}+\mu_{o w}\left(F_{\mu}{ }^{3}-F_{\mu}-F_{\mu}{ }^{2}\right)\right)+\mu_{o w}\left(\Psi_{2}+1\right) L_{p m}\right]\left(d L_{w g} / d t\right) \\
& \Delta p=\left(\phi_{2} / k_{2}\right)\left(1 / F_{\mu}\right)\left[\left(L_{f f} / F_{\mu}{ }^{2}\right)\left(\mu_{w g}+\mu_{o w}\left(F_{\mu}{ }^{3}-F_{\mu}-F_{\mu}{ }^{2}\right)\right)+\mu_{o w}\left(\Psi_{2}+1\right) L_{p m}\right]\left(d L_{f f} / d t\right)
\end{aligned}
$$




$$
\begin{aligned}
& \Delta p=\left(\phi_{2} / k_{2}\right)\left(1 / F_{\mu}\right)\left[\mu_{w g} L_{f f} / F_{\mu}{ }^{2}+\mu_{o w} F_{\mu}\left(L_{f f}-L_{f f} / F_{\mu}{ }^{2}\right)+\mu_{o w}\left(L_{p m}-L_{f f}\right)+\mu_{o w} \Psi_{2} L_{p m}\right]\left(d L_{f f} / d t\right) \\
& \Delta p=\left(\phi_{2} / k_{2}\right)\left(1 / F_{\mu}\right)\left[L_{f f} / F_{\mu}{ }^{2}\left(\mu_{w g}+\mu_{o w}\left(F_{\mu}^{3}-F_{\mu}-F^{2}{ }_{\mu}\right)\right)+\mu_{o w}\left(\Psi_{2}+1\right) L_{p m}\right]\left(d L_{f f} / d t\right) \\
& \left(\phi_{1} / k_{1}\right)\left[\left(\mu_{p 1}-\mu_{o w}\right) L_{p l}+\mu_{o w}\left(\Psi_{1}+1\right) L_{p m}\right] d L_{p l}= \\
& \left(\phi_{2} / k_{2}\right)\left(1 / F_{\mu}\right)\left[L_{f f}\left(1 / F_{\mu}{ }^{2}\right)\left(\mu_{w g}+\mu_{o w}\left(F_{\mu}{ }^{3}-F_{\mu}-F_{\mu}{ }^{2}\right)\right)+\mu_{o w}\left(\Psi_{2}+1\right) L_{p m}\right] d L_{f f} \\
& \left(\phi_{1} / k_{1}\right)\left[\left(\mu_{p 1}-\mu_{o w}\right) L_{p 1}{ }^{2} / 2+\mu_{o w}\left(\Psi_{1}+1\right) L_{p m} L_{p l}\right]= \\
& \left(\phi_{2} / k_{2}\right)\left(1 / F_{\mu}\right)\left[L_{f f}{ }^{2} / 2 F_{\mu}{ }^{2}\left(\mu_{w g}+\mu_{o w}\left(F_{\mu}{ }^{3}-F_{\mu}-F_{\mu}{ }^{2}\right)\right)+\mu_{o w}\left(\Psi_{2}+1\right) L_{p m} L_{f f}\right]= \\
& \left(\phi_{1} / k_{1}\right)\left[\left(\mu_{p 1}-\mu_{o w}\right) L_{p m}{ }^{2} / 2+\mu_{o w}\left(\Psi_{1}+1\right) L_{p m}{ }^{2}\right]=\left(\phi_{1} / k_{1}\right) L_{p m}{ }^{2}\left[\mu_{p 1} / 2+\mu_{o w} / 2+\mu_{o w} \Psi_{1}\right] \\
& \left(k_{2} \phi_{1} F_{\mu} / k_{1} \phi_{2}\right) L_{p m}{ }^{2}\left[\left(\mu_{p 1}+\mu_{o w} / 2\right)+\mu_{o w} \Psi_{1}\right]=\left[\mu_{o w}\left(\Psi_{2}+1\right)\left(L_{p m}\right)\right] L_{f f}+ \\
& {\left[\mu_{w g}+\mu_{o w}\left(F_{\mu}{ }^{3}-F_{\mu}{ }^{2}-F_{\mu}\right)\right] /\left[2 F_{\mu}{ }^{2}\right] L_{f f}{ }^{2}}
\end{aligned}
$$

\section{Radial Flow Foam:}

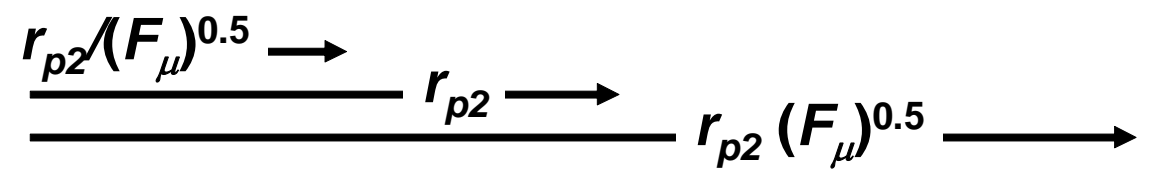

\section{Zone 2}

\section{Zone 1}

Fig. C-2-Gas/water flow in Zone 2 after foam forms in Zone 1. No Crossflow. Radial flow.

From Refs. 129 and 165:

$$
\begin{aligned}
& r_{w g}=r_{p f} / \sqrt{\mathrm{F}_{\mu}} \\
& r_{f f}=r_{p f} \sqrt{\mathrm{F}_{\mu}} \\
& r_{w g}=r_{f f} / F_{\mu}
\end{aligned}
$$




\section{Zone 2}

$\Delta p=\left(\phi_{2} / k_{2}\right) r_{p 2}\left(d r_{p 2} / d t\right)\left[\mu_{w g} \ln \left(r_{w g} / r_{o}\right)+\mu_{o w} F_{\mu} \ln \left(F_{\mu}\right)+\mu_{o w} \ln \left(r_{p m} / r_{f f}\right)+\mu_{o w} \Psi_{2} \ln \left(r_{p m} / r_{o}\right)\right]$

$\Delta p=\left(\phi_{2} / k_{2}\right) r_{p 2}\left(d r_{p 2} / d t\right)\left[\mu_{w g} \ln \left(r_{p 2} /\left(r_{o} \sqrt{\mathrm{F}_{\mu}}\right)\right)+\mu_{o w} F_{\mu} \ln \left(F_{\mu}\right)+\mu_{o w} \ln \left(r_{p m} /\left(r_{p 2} \sqrt{\mathrm{F}_{\mu}}\right)\right)+\right.$ $\left.\mu_{o w} \Psi_{2} \ln \left(r_{p m} / r_{o}\right)\right]$

$\Delta p=\left(\phi_{2} / k_{2}\right)\left[\mu_{w g} \ln r_{p 2}-\mu_{w g} \ln \left(r_{o} \sqrt{\mathrm{F}_{\mu}}\right)+\mu_{o w} F_{\mu} \ln \left(F_{\mu}\right)+\mu_{o w} \ln \left(r_{p m} / \sqrt{\mathrm{F}_{\mu}}\right)-\right.$ $\left.\mu_{o w} \ln \left(r_{p 2}\right)+\mu_{o w} \Psi_{2} \ln \left(r_{p m} / r_{o}\right)\right] r_{p 2}\left(d r_{p 2} / d t\right)$

$\Delta p=\left(\phi_{2} / k_{2}\right)\left[r_{p 2} \ln r_{p 2}\left(\mu_{w g}-\mu_{o w}\right)+r_{p 2}\left(\mu_{o w} \Psi_{2} \ln \left(r_{p m} / r_{o}\right)+\mu_{o w} F_{\mu} \ln \left(F_{\mu}\right)-\right.\right.$

$\left.\left.\mu_{w g} \ln \left(r_{o} \sqrt{\mathrm{F}_{\mu}}\right)+\mu_{o w} \ln \left(r_{p m} / \sqrt{\mathrm{F}_{\mu}}\right)\right)\right]\left(d r_{p 2} / d t\right)$

$\int x \ln (\beta x) d x=1 / 2\left[x^{2}\right] \ln (\beta x)-1 / 2 x^{2} / 2=\left(x^{2} / 2\right)[\ln (\beta x)-1 / 2]$

$\left(\phi_{2} / k_{2}\right)\left[\left(\mu_{w g}-\mu_{o w}\right)\left(r_{p 2}\right)^{2}\left(\ln \left(r_{p 2}\right)-1 / 2\right)+r_{p 2}{ }^{2}\left(\mu_{o w} \Psi_{2} \ln \left(r_{p m} / r_{o}\right)+\mu_{o w} F_{\mu} \ln \left(F_{\mu}\right)-\right.\right.$

$\left.\left.\mu_{w g} \ln \left(r_{o} \sqrt{\mathrm{F}_{\mu}}\right)+\mu_{o w} \ln \left(r_{p m} / \sqrt{\mathrm{F}_{\mu}}\right)\right)\right]\left.\right|_{r_{o}} ^{r_{p 2}}$

$=\left(\phi_{1} / k_{2}\right) \mu_{o w}\left[r_{p 1} \ln \left(r_{p 1}\right)\left(F_{r}-1\right)+r_{p 1}\left(\Psi_{1} \ln \left(r_{p m} / r_{o}\right)+\ln \left(r_{p m}\right)-F_{r} \ln \left(r_{o}\right)\right)\right] d r_{p 1} / d t$

$=\left(\phi_{1} / k_{2}\right)\left(\mu_{o w}\right)\left[\left(r_{p 1}\right)^{2}\left[\ln \left(r_{p 1}\right)-1 / 2\right]\left(F_{r}-1\right)+\left.\left(r_{p 1}\right)^{2}\left(\Psi_{1} \ln \left(r_{p m} / r_{o}\right)+\ln \left(r_{p m}\right)-F_{r} \ln \left(r_{o}\right)\right]\right|_{r_{o}} ^{r_{p 1}}\right.$

$=\left(\phi_{1} / k_{1}\right)\left(\mu_{o w}\right)\left[\left(r_{p 1}{ }^{2}\right)\left[\ln \left(r_{p l}\right)\left(F_{r}-1\right)+\left(\Psi_{1} \ln \left(r_{p m} / r_{o}\right)+\ln \left(r_{p m}\right)-F_{r} \ln \left(r_{o}\right)-\left(F_{r}-1\right) / 2\right)\right]\right.$

$\left(\phi_{2} / k_{2}\right)\left(r_{p 2}^{2}\right)\left[\left(\mu_{w g}-\mu_{o w}\right) \ln \left(r_{p 2}\right)-\left(\mu_{w g}-\mu_{o w}\right) / 2-\mu_{w g} \ln \left(r_{o} \sqrt{\mathrm{F}_{\mu}}\right)+\right.$

$\left.\mu_{o w}\left[\Psi_{2} \ln \left(r_{p m} / r_{o}\right)+F_{\mu} \ln \left(F_{\mu}\right)+\ln \left(r_{p m} / \sqrt{F_{\mu}}\right)\right]\right]$

$=\left(\phi_{1} / k_{1}\right)\left(\mu_{o w}\right)\left(r_{p 1}^{2}\right)\left[F_{r} \ln \left(r_{p 1} / r_{o}\right)+\ln \left(r_{p m} / r_{p l}\right)+\left(1-F_{r}\right) / 2+\Psi_{1} \ln \left(r_{p m} / r_{o}\right)\right]$

$\left.r_{p 2}^{2}=\left(\phi_{1} k_{2} / \phi_{2} k_{1}\right)\left(\mu_{o w}\right)\left(r_{p 1}^{2}\right)\left[\ln \left(r_{p 1} / r_{o}\right)\left[F_{r}+\Psi_{1}\right]+\left(1-F_{r}\right) / 2\right)\right] /$

$\left[\left(\mu_{w g}-\mu_{o w}\right)\left[\ln \left(r_{p 2}\right)-1 / 2\right]-\mu_{w g} \ln \left(r_{o} \sqrt{\mathrm{F}_{\mu}}\right)+\mu_{o w}\left[\Psi_{2} \ln \left(r_{p 1} / r_{o}\right)+F_{\mu} \ln \left(F_{\mu}\right)+\ln \left(r_{p m} / \sqrt{\mathrm{F}_{\mu}}\right)\right]\right]$

$\int x \ln \times d x=1 / 2\left[x^{2}\right][\ln x-1 / 2]$

$2 x[\ln x-1 / 2]+\left(x^{2}\right) / x$ 
$1 / 2\left[2 x[\ln x-1 / 2]+x^{2} / x\right]=x \ln x-x+x$

$\left(\phi_{2} / k_{2}\right)(1 / 2)\left(r_{p 2}\right)^{2}\left[\ln r_{p 2}-1 / 2\right]\left(\mu_{w g}-\mu_{o w}\right)+$

$\left.r_{p 2}^{2} / 2\right)\left[\mu_{o w} \Psi_{2} \ln \left(r_{p m} / r_{o}\right)+\mu_{o w} F_{\mu} \ln \left(F_{\mu}\right)-\mu_{w g} \ln \left(r_{o} \sqrt{\mathrm{F}_{\mu}}\right)+\mu_{o w} \ln \left(r_{p m} / \sqrt{\mathrm{F}_{\mu}}\right)\right]$

$r_{p 2}{ }^{2}\left[\left(\mu_{w g}-\mu_{o w}\right)\left[\ln \left(r_{p 2}\right)-1 / 2\right]+\mu_{o w}\left[\Psi_{2} \ln \left(r_{p 1} / r_{o}\right)+F_{\mu} \ln \left(F_{\mu}\right)+\ln \left(r_{p 1} / \sqrt{\mathrm{F}_{\mu}}\right)\right]-\right.$ $\left.\mu_{w g} \ln \left(r_{o} \sqrt{\mathrm{F}_{\mu}}\right)\right]$ 